\title{
The effects of membrane physicochemical properties on huntingtin membrane association and downstream aggregation
}

\author{
Maryssa Beasley \\ West Virginia University, mab0097@mix.wvu.edu
}

Follow this and additional works at: https://researchrepository.wvu.edu/etd

Part of the Analytical Chemistry Commons, Biochemistry Commons, Biophysics Commons, and the Physical Chemistry Commons

\section{Recommended Citation}

Beasley, Maryssa, "The effects of membrane physicochemical properties on huntingtin membrane association and downstream aggregation" (2020). Graduate Theses, Dissertations, and Problem Reports. 7968.

https://researchrepository.wvu.edu/etd/7968

This Dissertation is protected by copyright and/or related rights. It has been brought to you by the The Research Repository @ WVU with permission from the rights-holder(s). You are free to use this Dissertation in any way that is permitted by the copyright and related rights legislation that applies to your use. For other uses you must obtain permission from the rights-holder(s) directly, unless additional rights are indicated by a Creative Commons license in the record and/ or on the work itself. This Dissertation has been accepted for inclusion in WVU Graduate Theses, Dissertations, and Problem Reports collection by an authorized administrator of The Research Repository @ WVU.

For more information, please contact researchrepository@mail.wvu.edu. 


\title{
The effects of membrane physicochemical properties on
}

\section{huntingtin membrane association and downstream aggregation}

\author{
Maryssa Beasley \\ Dissertation submitted to the \\ Eberly College of Arts and Science \\ at West Virginia University
}

in partial fulfillment of the requirements for the degree of

Doctor of Philosophy

in

Chemistry

Justin Legleiter, Ph.D., Committee Co-Chairperson

Stephen Valentine, Ph.D., Committee Co-Chairperson

Blake Mertz, Ph.D.

Glen Jackson, Ph.D.

Werner Geldenhuys, Ph.D.

C. Eugene Bennett Department of Chemistry

Morgantown, West Virginia

2020

Keywords: Huntington's Disease, amyloid formation, polydiacetylene assay, aggregation inhibitors, anionic lipids, lipid tail unsaturation, amphipathic $\alpha$-helix, native mass spectrometry, WESTPA

Copyright 2020 Maryssa Beasley 


\begin{abstract}
The effects of membrane physicochemical properties on huntingtin membrane association and downstream aggregation
\end{abstract}

\author{
Maryssa Beasley
}

Huntington's Disease (HD) is a fatal neurodegenerative disorder caused by an expanded glutamine repeat region (polyQ) within the huntingtin protein (htt). As a result of the expanded polyQ domain, htt associates into a variety of toxic aggregate species. The polyQ domain of htt is flanked at the N-terminal end by 17 amino acids (Nt17) that adopt an amphipathic $\alpha$-helical structure in the presence of binding partners such as lipid membranes. In addition to comprising a lipid binding domain, the Nt17 amphipathic $\alpha$-helix has been directly implicated in htt aggregation initiation via self-association with other Nt17 $\alpha$-helices. Due to this, htt/lipid interaction likely has a large impact on the rate and extent of htt aggregate formation, with potential implications for HD pathogenesis. The studies presented here focus on elucidating the effect of membrane physicochemical properties on htt membrane association and downstream htt aggregation. In order to measure membrane association, a method of normalizing polydiacetylene (PDA) lipid binding assays was developed to enable the direct comparison of various molecules' binding affinity for different lipid systems. Then, the normalized PDA assay was utilized to determine if small molecule aggregation inhibitors influence the interaction of htt with pure and physiologically relevant lipid systems, and thioflavin-T (ThT) assays and atomic force microscopy (AFM) were used to monitor if the interaction altered the ability of the small molecules to inhibit htt aggregation. While both small molecules altered htt-membrane 
association, EGCG remained an effective aggregation inhibitor while curcumin no longer inhibited htt fibrillization in the presence of either lipid system. These results highlight the complex relationship between htt-membrane association, downstream aggregation, and the ability of small molecules to inhibit htt aggregation in a cellular environment. Subsequent studies utilized ThT, AFM, polydiacetylene (PDA) assays, and native MS to determine how altering membrane physicochemical properties by changing the headgroup or the tail of lipids influences htt aggregation and membrane affinity. Our results indicate that increasing the negative charge of lipid headgroups disrupts the insertion of $\mathrm{Nt} 17$ into the bilayer, which in turn decreases membrane disruption and results in a localization effect that increases htt fibrillization. Also, when varying the degree of unsaturation in the lipid tail, the trend of htt aggregation in the presence of each lipid system is different than the trend of association between htt and the vesicles, indicating that membrane properties alter the mechanism of downstream htt aggregation. Further investigation of the interaction between Nt17 and lipid membranes with molecular dynamics (MD) simulations reveal that a combination of the hydrophobic amino acid and available membrane defect sizes influence the orientation of $\mathrm{Nt} 17$ on bilayers during the initial stages of interaction. Collectively, these results highlight how the properties of lipid membranes modulate htt-membrane interactions and htt aggregation mechanisms. 


\section{Acknowledgements}

The completion of the studies presented here would not have been possible without the support, guidance, and assistance of many individuals. Each individual proved monumental towards the success of these studies, and I would like to express my sincerest thanks and deepest gratitude to those individuals now.

Joining the labs of Dr. Justin Legleiter and Dr. Stephen Valentine was truly a blessing, and I am grateful that they provided me the opportunity to develop my skills as a researcher and grow as a scientist. Because of the mentorship of my advisors, I was given the unique opportunity to combine my love for analytical chemistry with my growing curiosity for physical chemistry. I will forever cherish the intellectual and thought-provoking discussions I got to have with Dr. Legleiter. His passion for this field truly is contagious, as is his humor. I am also constantly in awe of Dr. Valentine's mind and the ideas that he can come up with. I am thankful to them both for their guidance and patience throughout the years. Most people are lucky to have one advisor like them, and I was fortunate enough to have two.

I am exceptionally grateful to every professor, mentor, and collaborator who provided guidance throughout my academic career. I will be forever thankful to Dr. Peter Harrington for offering me my first research position as an undergraduate, and to Dr. Becky Barlag and Dr. Lauren McMills for their academic guidance and support at Ohio University. A sincere thank you to The Ruby Distinguished Doctoral Fellows Program for believing in me from the start and providing funding that allowed me to focus exclusively on my research at West Virginia University. I would also like to thank my committee members Dr. Blake Mertz, Dr. Glen Jackson, and Dr. Werner Geldenhuys for their advice and feedback throughout my graduate 
career. I am particularly grateful to Dr. Mertz for the shared expertise, knowledge, and ideas during our collaboration. Finally, I would like to thank my lab mates for the assistance, encouragement, and friendship.

I would also like to thank my mom and dad for the immense amounts of support throughout the years and for encouraging me to pursue my passions. None of this would have been possible without you two. In addition, I would like to thank the rest of my family for everything they have done to support my journey: my grandmother Barb Brunt for being a role model of a strong, intelligent woman and passing on her love for learning; Becky and Steve Pantuso for being the loudest voice of support my whole life, from cheering at childhood soccer games to cheering at college graduations; Sam and Dominick for bringing light and fun to every gathering; and Jami, for being the kindest, sweetest cheerleader anyone could ask for.

This also would not have been possible without the truly amazing friends I made during this long, long journey: Steven Kolich and Shannon McCall, who have stood by my side and made life exciting since childhood; Nikki Streidl, Jacob Fadel, Trish Seigfried, and Alexis Benedis for filling my life with so much support and laughter since undergraduate; Dr. Daniel Nething for teaching me that between teaching, classes, and research, scientists can still have fun; and Sharon Groover, Nicolas Frazee, and Blaine McClay for countless "Fam Dinners" and game nights that kept me sane during graduate school. A special thank you goes to Sharon Groover for being my guide, collaborator, and partner in crime, in and out of the lab.

Finally, I would like to thank my fiancé, Paymon Doroodian. You have always believed in me, and your unconditional love and support mean the world. Thank you for being my rock, sounding board, therapist, and absolute best friend. 


\section{Table of Contents}

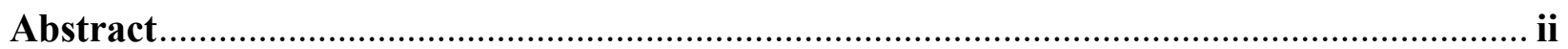

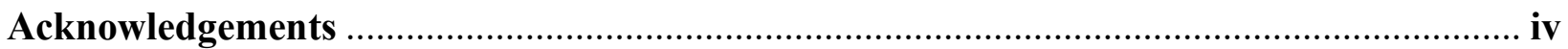

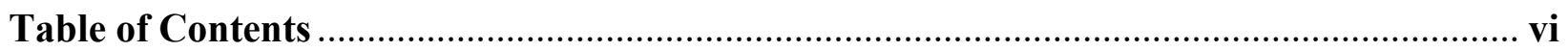

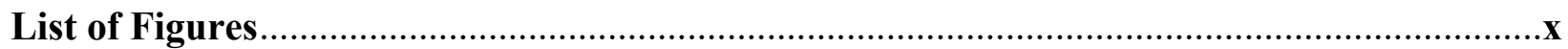

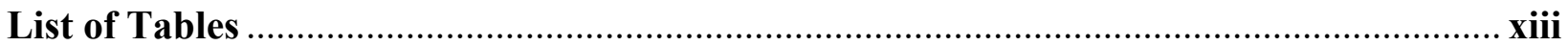

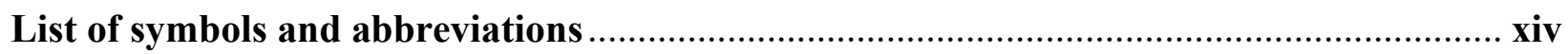

1. Introduction: Structure and Functions of Huntingtin, the Protein Associated with

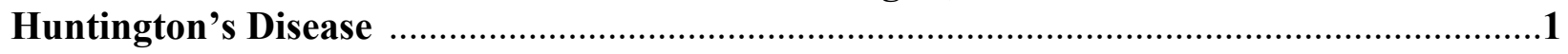

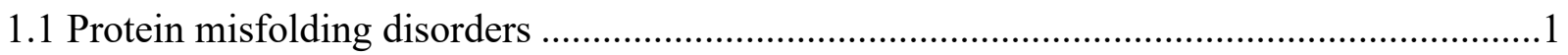

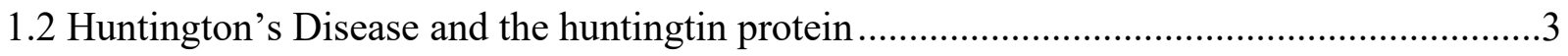

1.3 Huntingtin aggregation mechanisms and toxicity ………….............................................

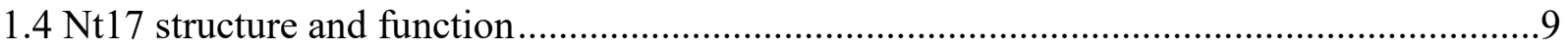

1.5 Functions and toxic events associated with htt-lipid interaction ........................................12

1.6 The influence of membranes on huntingtin aggregation.................................................15

1.7 Combination of experimental approaches to study huntingtin at different scales ...............16

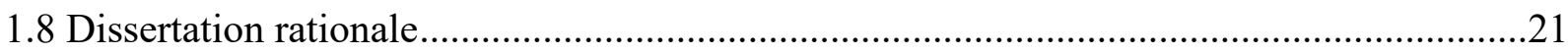

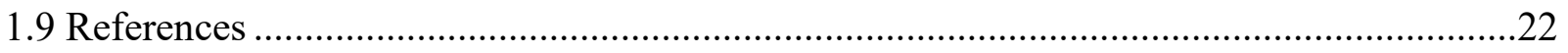

2. Normalizing Polydiacetylene Colorimetric Assays of Vesicle Binding Across Lipid

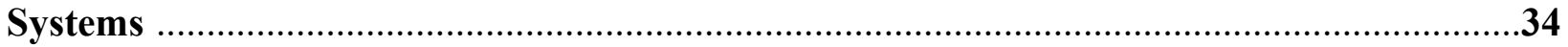

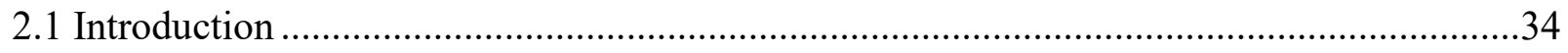

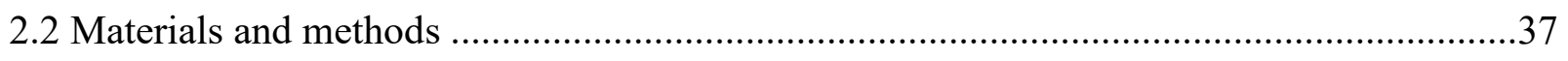

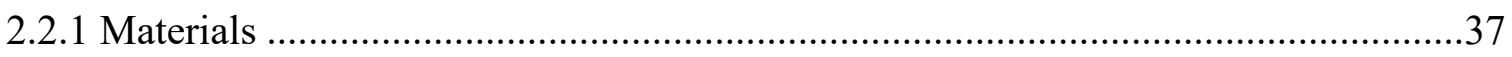

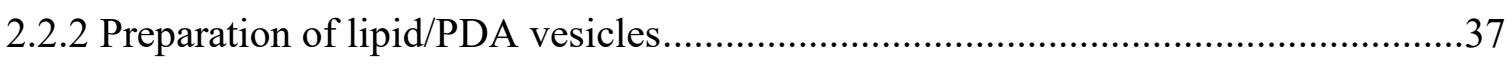

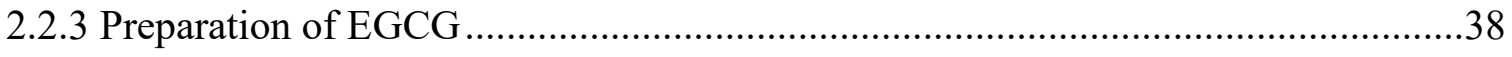


2.2.4 Preparation of $\beta$-amyloid peptide

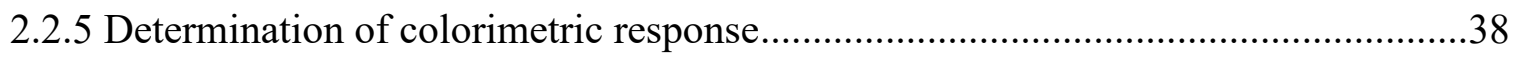

2.2.6 Assay normalization and analysis ............................................................................39

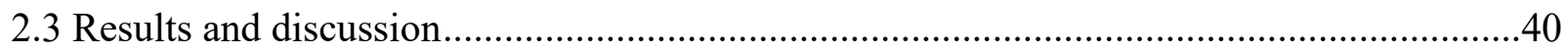

2.3.1 Colorimetric response is linearly correlated to applied stress .................................40

2.3.2 Calibration and normalization of colorimetric response enhances cross-batch and

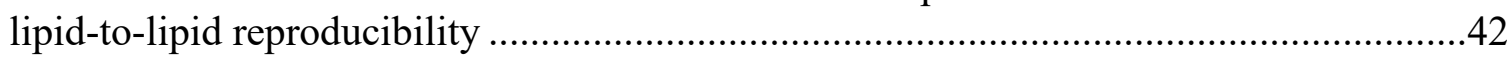

2.3.3 Normalized PDA assays can determine relative binding affinities of small molecules and peptides to lipid membranes ........................................................................4

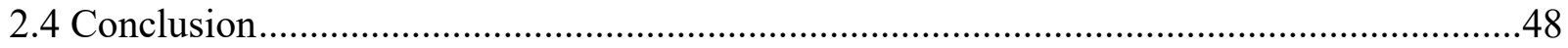

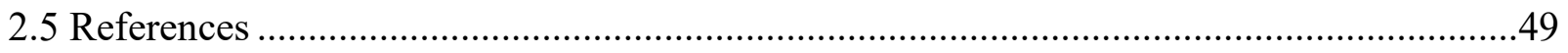

3. Lipid membranes influence the ability of small molecules to inhibit huntingtin

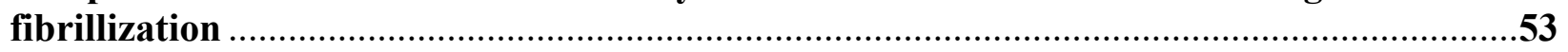

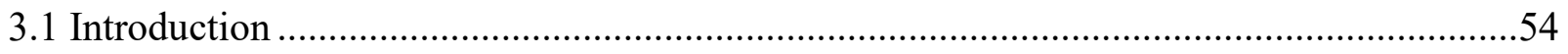

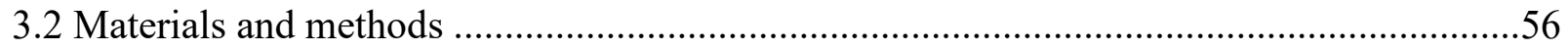

3.2.1 Purification of GST-htt-exon1 fusion protein..........................................................56

3.2.2 Preparation of lipid vesicles.................................................................................57

3.2.3 Thioflavin T aggregation assays ........................................................................57

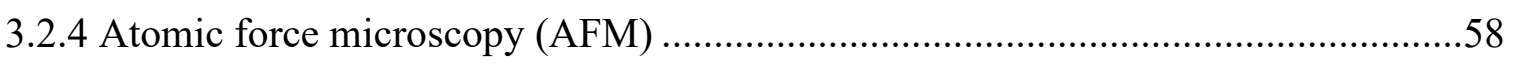

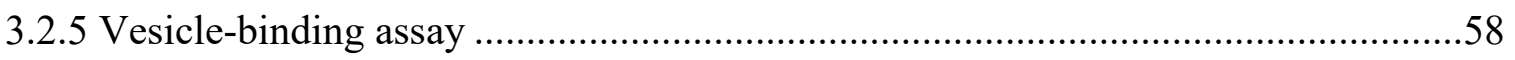

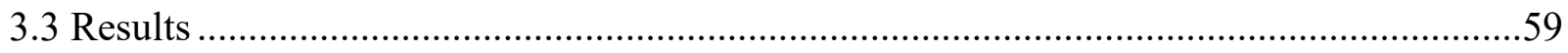

3.3.1 Curcumin and EGCG inhibit htt fibrillization .......................................................59

3.3.2 Lipid membranes alter the ability of curcumin and EGCG to inhibit fibrillization..67

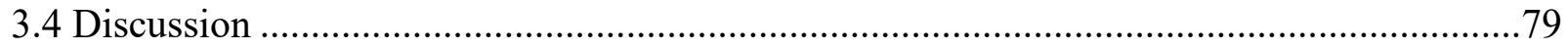

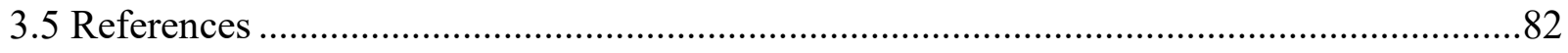

4. Lipid headgroups alter huntingtin aggregation on membranes.......................................89 


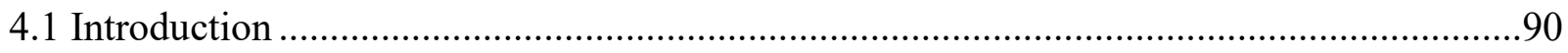

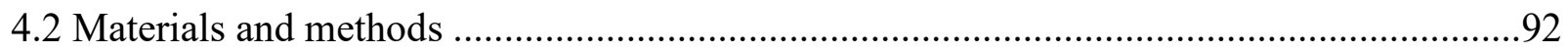

4.2.1 Purification of glutathione S-transferase (GST)-htt-exon1 fusion protein ................92

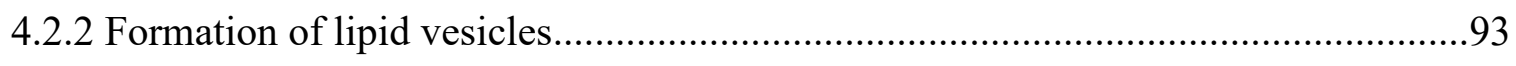

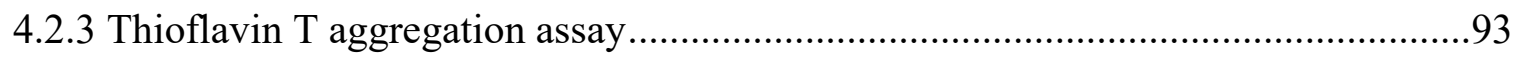

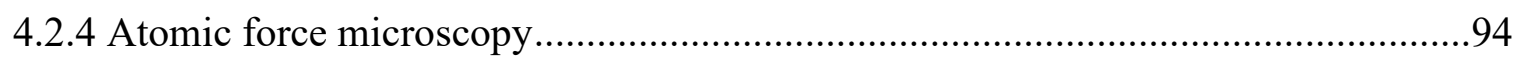

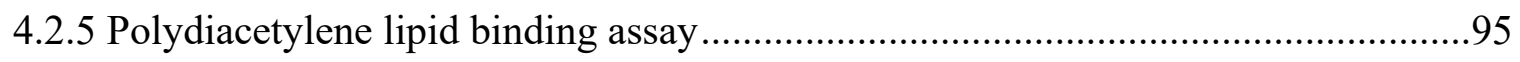

4.2.6 Electrospray ionization-mass spectrometry (ESI-MS) …………………………....96

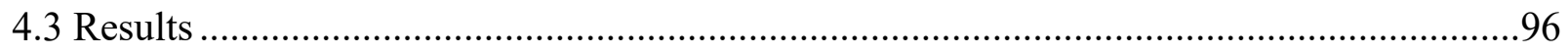

4.3.1 Lipids modify htt aggregation to various extents .................................................96

4.3.2 Htt has varying affinity for different lipid systems...............................................105

4.3.3 Vesicle charge alters the complexes formed between the lipid binding domain of htt

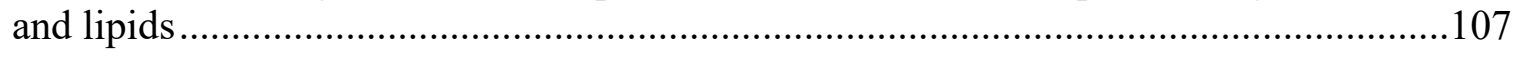

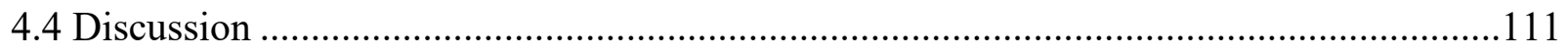

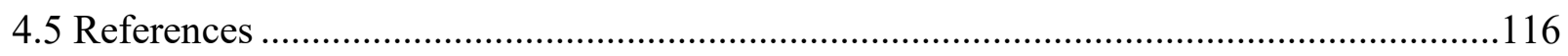

\section{Physicochemical Properties Altered by the Tail Group of Lipid Membranes Influence}

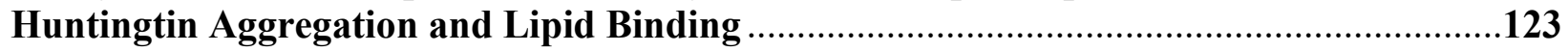

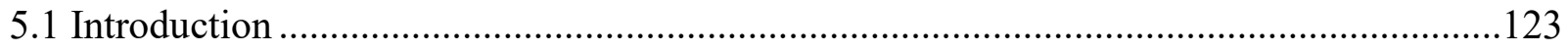

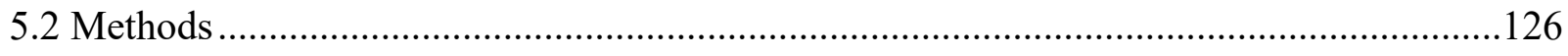

5.2.1 Purification of glutathione S-transferase (GST)-htt-exon1 fusion protein ..............126

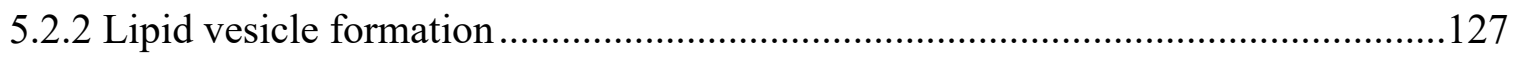

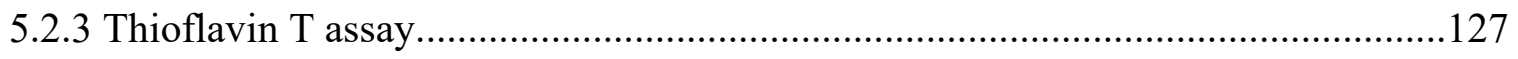

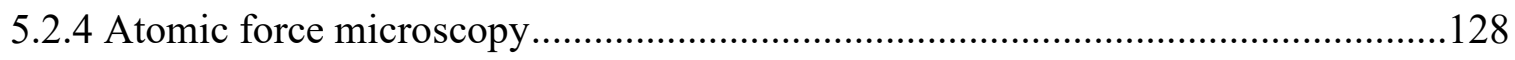

5.2.5 Polydiacetylene lipid binding assay ..................................................................128

5.2.6 Electrospray ionization-mass spectrometry (ESI-MS) ………................................129

5.2.7 Molecular dynamics system preparation ..............................................................130 


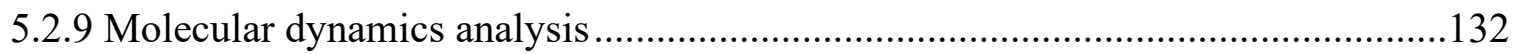

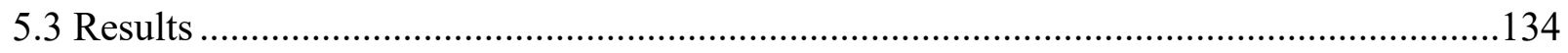

5.3.1 Lipid membranes impact htt aggregation ...........................................................134

5.3.2 Saturation in lipid tails influence htt/membrane interactions ................................140

5.3.3 Nt17 forms a variety of complexes with lipids ....................................................141

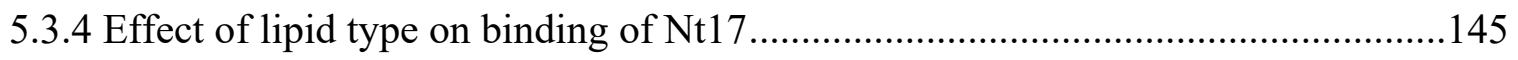

5.3.5 Relationship between sidechain orientation and binding of $\mathrm{Nt17}$.........................147

5.3.6 The role of protrusions and defects in binding and stabilization of Nt17..............149

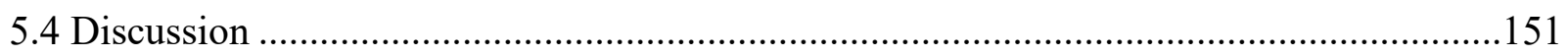

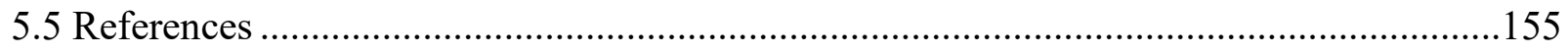

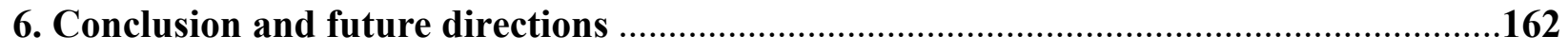

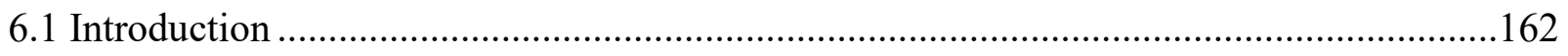

6.2 Research in progress: Elucidating the effect of membrane cholesterol content on htt aggregation and membrane affinity ......................................................................................16

6.3 Identifying the interaction of specific aggregate species with lipid membranes ...............171

6.4 A systematic approach to understand amphipathic $\alpha$-helix membrane selectivity ............175

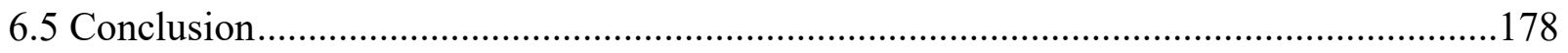

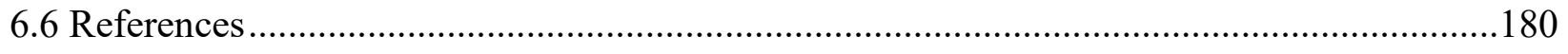




\section{List of Figures}

1. An Introduction to Huntingtin: Characteristics, Functions, and Mechanisms of the Protein Associated with Huntington's Disease ........................................................

1.1 Schematic of a combined energy landscape for protein folding and aggregation ...............2

1.2 The mutation that results in mutant, pathogenic htt protein ...........................................

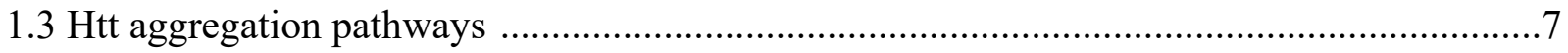

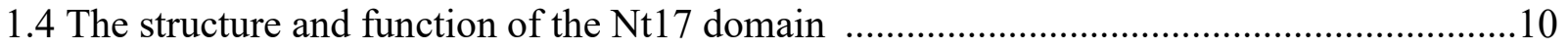

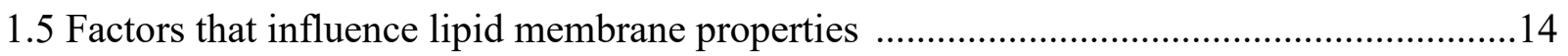

1.6 Electrospray ionization produces gas-phase ions from solution ...................................17

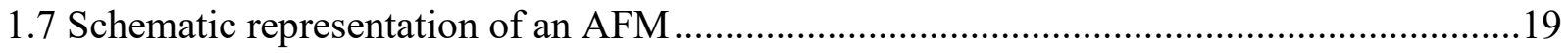

2. Normalizing Polydiacetylene Colorimetric Assays of Vesicle Binding Across Lipid

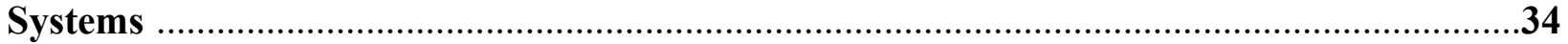

2.1 PDA normalization reduces assay variability ..........................................................41

2.2 The response of PDA/lipid vesicles to $\mathrm{NaOH}$ varies batch to batch ...............................41

2.3 PDA/lipid vesicle exhibit a colorimetric response upon exposure to EGCG and A $\beta$ peptide

2.4 DMSO and riluzole do not elicit a colorimetric response

\section{Lipid membranes influence the ability of small molecules to inhibit huntingtin} fibrillization

3.1 Curcumin and EGCG decrease htt fibrillization

3.2 Small molecules alone or with lipid do not result in a ThT signal .................................61

3.3 The effect of small molecules on htt fibrillization is consistent across all experiments .....61

3.4 AFM analysis of the impact of curcumin or EGCG on htt-exon1(46Q) aggregation ........63

3.5 Small molecules and lipids result in AFM backgrounds with low RMS roughness ..........64

3.6 EGCG alters htt oligomer morphology ..................................................................65

3.7 Htt aggregate morphology is altered by curcumin and EGCG ...................................66 
3.8 POPC and TBLE alter the ability of small molecules to inhibit htt-exon1(46Q)

fibrillization

3.9 POPC and TBLE vesicles were comparable in size

3.10 AFM analysis of the impact of curcumin or EGCG on htt-exon1(46Q) aggregation in the presence of POPC lipid vesicles .70

3.11 AFM analysis of the impact of curcumin or EGCG on htt-exon1(46Q) aggregation in the presence of TBLE vesicles

3.12 Curcumin and EGCG alter htt-exon1(46Q) aggregate morphology in the presence of lipid vesicles

3.13 EGCG promotes large oligomeric aggregates of htt-exon1(46Q) in the presence of POPC

3.14 EGCG promotes large oligomeric aggregates of htt-exon1(46Q) in the presence of TBLE

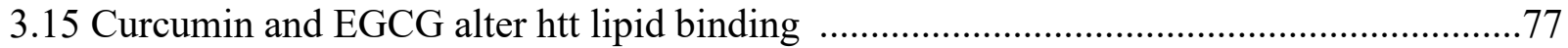

3.16 Individual trials of the PDA lipid binding assays are consistent .................................77

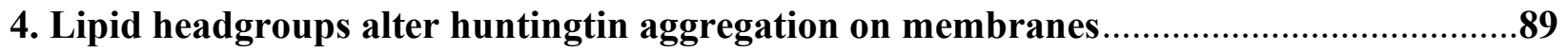

4.1 Varying the lipid headgroup alters how vesicles influence htt fibrilization.......................98

4.2 Representative AFM images showing the effect of different lipids on htt-exon1(46Q) aggregation 100

4.3 AFM images of lipid backgrounds

4.4 AFM analysis of the impact of different lipid systems on htt-exon1(46Q) aggregation ...102

4.5 POPG vesicles promote a unique htt-exon1(46Q) fibril morphology 103

4.6 Htt-exon1(46Q) membrane association is altered by lipid headgroup 106

4.7 Nt17 forms complexes with lipids that are observable by native MS 108

4.8 Populations of complexes formed with $\mathrm{Nt17}$ and lipid are altered by the lipid headgroup

\section{Physicochemical Properties Altered by the Tail Group of Lipid Membranes Influence Huntingtin Aggregation and Lipid Binding}


5.2 Lipid vesicles alone do not induce ThT fluorescence

5.3 Varying the lipid tail alters how vesicles influence htt fibrilization 135

5.4 AFM images showing the effect of DMPC, POPC, and DOPC on htt-exon1(46Q) aggregation

5.5 AFM images of backgrounds associated with different lipids

5.6 AFM analysis of the impact of DMPC, POPC, or DOPC lipid vesicles on htt-exon1(46Q) aggregation 138

5.7 Htt-exon1(46Q) fibrils formed in the presence of DMPC, POPC, and DOPC vesicles are similar in morphology to those formed in the absence of lipid.

5.8 Htt-exon1(46Q) membrane interaction is altered by properties associated with lipid tail 140

$5.9 \mathrm{Nt} 17$ forms complexes with DMPC, POPC, or DOPC vesicles of different makeup and abundance as a result of varying molecular contacts.

5.10 Lipid tails affect the binding orientation of $\mathrm{Nt} 17$ to lipid bilayers

5.11 Secondary populations of Nt17 orientation on the lipid bilayers

$5.12 \mathrm{Nt} 17$ binding is facilitated via partitioning of amino acid sidechains into bilayer systems with higher areas per lipid 148

5.13 Lipid tails influence the partitioning of Nt17 amino acid sidechains into the bilayer ....149

5.14 Formation of stable proteolipid complexes occurs via contributions from both redistribution of bilayer deformations and stable intermediates of $\mathrm{Nt} 17$

6. Conclusion and future directions 162

6.1 Schematic presenting a method for PDA lipid binding assay normalization 163

6.2 Lipid membranes influence the efficiency of small molecules aggregation inhibition .....164

6.3 Mechanisms of htt lipid binding and aggregation are altered by membrane charge .165

6.4 Cholesterol alters the effect of lipid vesicles on htt aggregation 168

6.5 The impact of cholesterol on a membrane's effect on htt aggregation and lipid binding is dependent on the physicochemical properties of the pure lipid system.

6.6 Proposed experimental protocol for studying the association of specific htt aggregates with lipids of varying physicochemical properties. 


\section{List of Tables}

Table 2.1 Maximum S/N for three batches of each PDA/lipid system ........................................42

Table 2.2 Batch to batch relative standard deviation (RSD) values for each PDA/lipid system before and after normalization as a function of $\mathrm{NaOH}$ added

Table 2.3 Between-system relative standard deviation (RSD) values for all PDA/lipid systems before and after normalization as a function of $\mathrm{NaOH}$ added ....................................................44

Table 4.1 Assigned ions for Nt17 incubated with POPC, POPE, POPG, or POPS lipid vesicles along with their mass to charge ratios

Table 5.1 Assigned ions for Nt17 incubated with DMPC, POPC, or DOPC lipid vesicles along with their mass to charge ratios .143 


\section{List of Symbols and Abbreviations}

$\alpha$-syn

$\mathrm{A} \beta$

$\mathrm{A} \beta_{40}$

$\mathrm{AD}$

AFM

$\mathrm{AH}$

ALPS

Chol

CR

DLS

DMSO

EGCG

EM

ER

ESI

GM1

GST

HD

HESI

HFIP

hIAPP

HPLC

$\mathrm{Htt}$ $\alpha$-synuclein

$\beta$-amyloid

Synthetic $\beta$-amyloid 1-40 peptide

Alzheimer's Disease

Atomic force microscopy

Amphipathic $\alpha$-helix

Amphipathic lipid packing sensor

Cholesterol

Colorimetric response

Dynamic light scattering

Dimethyl sulfoxide

Epigallocatechin 3-gallate

Electron microscopy

Endoplasmic reticulum

Electrospray ionization

Ganglioside

Glutathione S-transferase

Huntington's Disease

Heated electrospray ionization

Hexafluoroisopropanol

Human islet amyloid polypeptide

High-performance liquid chromatography

Huntingtin protein 


\begin{tabular}{|c|c|}
\hline Htt-exon1(46Q) & Huntingtin exon 1 with 46 glutamines \\
\hline IAPP & Islet amyloid polypeptide \\
\hline IPTG & Isopropyl-thio-galactopyranoside \\
\hline LC & Liquid chromatography \\
\hline LOOS & Lightweight object-oriented structure library \\
\hline LUV & Large unilamellar vesicle \\
\hline MD & Molecular dynamics \\
\hline MS & Mass spectrometry \\
\hline NAMD & Nanoscale molecular dynamics \\
\hline Nt17 & First $17 \mathrm{~N}$-terminal amino acids of htt \\
\hline NPT ensemble & Constant-temperature, constant-pressure ensemble \\
\hline NVT ensemble & Constant-temperature, constant-volume ensemble \\
\hline PD & Parkinson's Disease \\
\hline PDA & Polydiacetylene \\
\hline Polyp & polyproline \\
\hline polyQ & polyglutamine \\
\hline PTMs & Post-translational modifications \\
\hline $\mathrm{R} 6 / 2$ mice & Transgenic mouse line that expresses htt-exon 1 with $120 \mathrm{Q}$ \\
\hline RMS & Root mean squared \\
\hline RSD & Relative standard deviation \\
\hline $\mathrm{S} / \mathrm{N}$ & Signal to noise ratio \\
\hline SM & Sphingomyelin \\
\hline SPR & Surface plasmon resonance \\
\hline SUV & Small unilamellar vesicle \\
\hline TBLE & Total brain lipid extract \\
\hline
\end{tabular}


ThT

TIRF

VMD

WESTPA
Thioflavin $\mathrm{T}$

Total internal reflection fluorescence

Visual molecular dynamics

Weighted ensemble simulation toolkit with parallelization and analysis 


\section{Introduction: Structure and Functions of Huntingtin, the Protein Associated with Huntington's Disease}

\subsection{Protein misfolding disorders}

A defining characteristic of numerous neurodegenerative disorders, including Alzheimer's Disease (AD), Parkinson's Disease (PD), and Huntington's Disease (HD), is the ordered accumulation of proteinaceous material in cellular compartments and tissues. In each of these diseases, a specific protein misfolds and associates into toxic aggregate species that result in pathogenesis. As a result, these diseases present clinically with impaired motor and cognitive function, ${ }^{1-3}$ while they present pathologically with neuronal cell loss and synaptic abnormalities. ${ }^{4}$ The hallmark feature of each of these neurodegenerative diseases is the conformational rearrangement of a protein that results in the aggregation and accumulation of amyloids in the brain. ${ }^{5,6}$ Amyloids are organized in a cross- $\beta$ structure, fibrillar in morphology, resistant to proteolytic degradation, and SDS insoluble. $^{7}$

These amyloid disorders, classified also as protein misfolding disorders, are associated with the misfolding of a normally functional protein. The typical progressive folding of proteins is illustrated in the concept of a folding energy landscape (Fig. 1.1). ${ }^{8,9}$ Generally, newly synthesized proteins progress along an energy landscape and fold into a minimal-energy conformation, or native conformation, dictated by their amino acid sequence. In this conformation, the hydrophobic amino acids are shielded from water molecules while the hydrophilic residues are exposed at the surface, minimizing the overall free energy of the protein. ${ }^{10,11}$ The energy landscape leading to the native conformation generally gets rougher based on the size of the protein in question, as longer amino acid sequences can fall into 
partially-folded intermediate species that represent a local minima in the energy landscape. ${ }^{12}$ In the case of protein misfolding disorders, the protein follows the wrong folding pathway or energy-minimizing funnel and gets trapped in a local minima, resulting in a misfolded conformation that is different than the native structure.

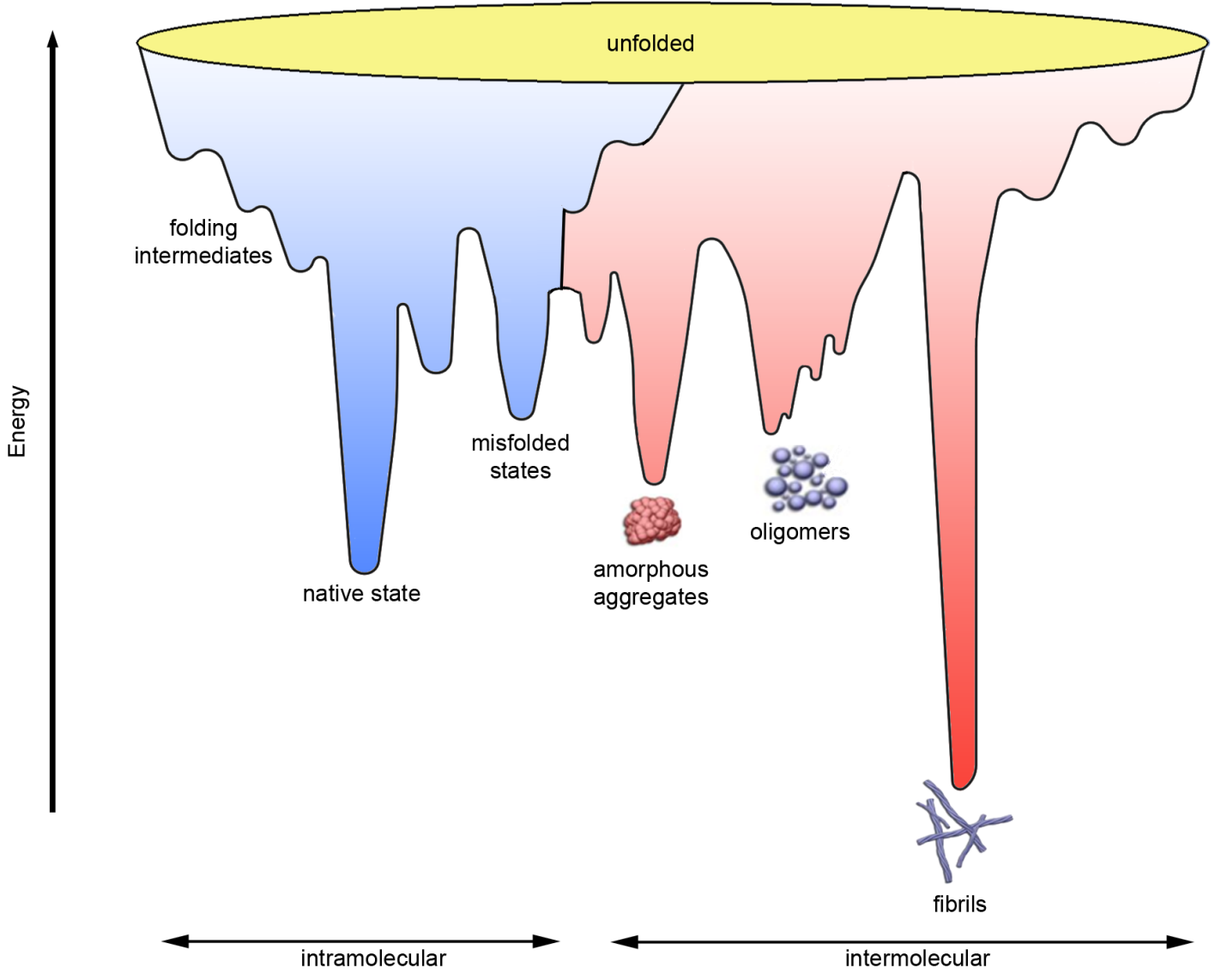

Figure 1.1 Schematic of a combined energy landscape for protein folding and aggregation. Unfolded proteins follow a protein-folding funnel to a minimized native-state folded structure (blue). Occasionally, a monomer does not follow the native folding pathway and instead results in a misfolded intermediate structure. With amyloid proteins, these misfolded states expose residues that encourage intermolecular interactions and result in aggregation into a variety of species (red).

In protein misfolding disorders, the misfolded conformations are often partially folded and expose aggregation-prone regions of the protein, increasing the occurrence of intermolecular 
interactions. ${ }^{13}$ Intermolecular interactions encourage the transition from the folding energy landscape to the aggregation energy landscape, where aggregate species such as annular aggregates, oligomers, and fibrils are formed. Due to the combination of intramolecular and intermolecular interactions in the aggregation landscape, a large variety of conformations and aggregate species form that have broad ensembles of sizes and states as well as poorly defined energy minima. ${ }^{13}$ With amyloidogenic proteins, a combination of misfolding and increased intermolecular interactions often results in monomeric or multimeric structures rich in $\beta$-sheets, which expose hydrophobic amino acid residues in the protein and promotes the formation of amyloid-like fibrils. ${ }^{14}$ While the depth of the fibril well in the aggregation energy landscape varies greatly based on the protein and the fibril structure, the energy minima is often deeper and sharper than the native monomeric protein, making it exceptionally thermodynamically stable. ${ }^{15}$ At this point, the balance of the protein synthesis, folding, and degradation is disturbed, resulting in the accumulation of the protein aggregates in tissues. ${ }^{16}$

\subsection{Huntington's Disease and the huntingtin protein}

HD is a fatal neurodegenerative disorder that presents with symptoms of chorea, dystonia, and cognitive deficiencies. ${ }^{17}$ This autosomal dominant genetic disease is caused by a mutation in the IT15 gene found on the short arm of chromosome 14 that codes for the huntingtin protein (htt) (Fig. 1.2). The mutation is an expanded region of CAG trinucleotide repeats, which codes for an expanded polyglutamine (polyQ) domain near the N-terminus of htt. While the normal length of the htt polyQ domain is in the range of 17-20 repeats, expansion of this domain beyond 35 repeats is associated with disease. ${ }^{18,19}$ This is largely due to the association of longer polyQ chains with lower free energy penalties for $\beta$-rich structure formation. ${ }^{20,21}$ As a result, longer polyQ chains are more likely to undergo misfolding and are 
associated with increased aggregate formation and lower ages of HD onset. ${ }^{18,19,22}$ Late onset $(>50$ years old) occurs in individuals with a median repeat length of 42, typical onset (21-50 years old) occurs with median repeat lengths of 45, and juvenile onset (2-20 years old) occurs in individuals with median repeat length of 60 glutamines. ${ }^{22}$ This polyQ-dependent age of onset is a result of toxic htt aggregate formation, as pathogenic htt misfolds in a polyQ-dependent manner ${ }^{23}$ into a variety of aggregate species that result in the onset of Huntington's Disease. ${ }^{18,19}$

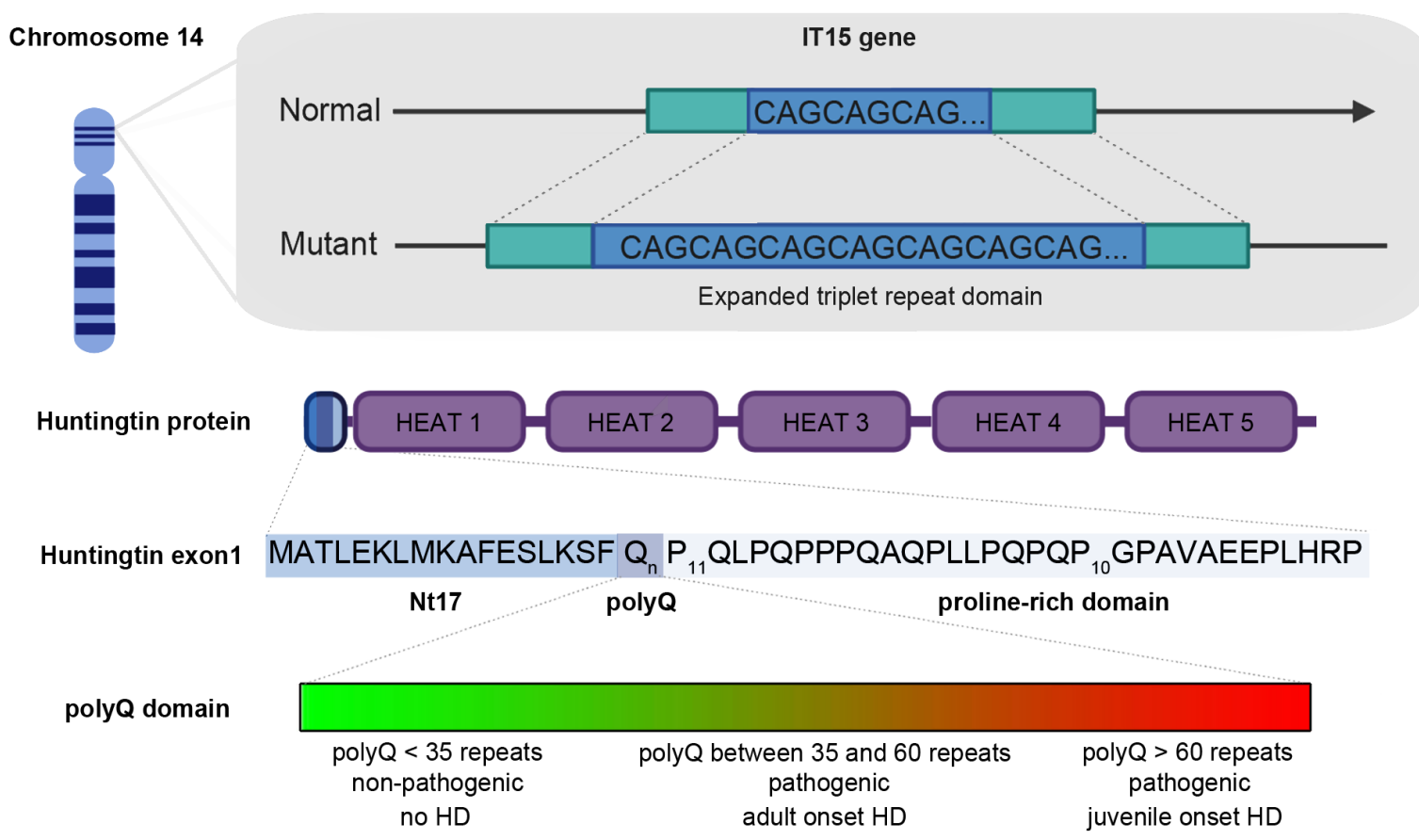

Figure 1.2 The mutation that results in mutant, pathogenic htt protein. ${ }^{157}$ The IT15 gene that codes for the huntingtin protein is found on the short arm of chromosome 14. An expanded CAG triplet repeat domain in the IT15 gene codes is responsible for the mutation in $\mathrm{htt}$ that results in pathogenicity. The mutation is in an N-terminal domain of htt called htt exon1. Exon is comprised of three domains: the $17 \mathrm{~N}$-terminal amino acids (Nt17), the glutamine repeat domain (polyQ), and a proline-rich domain. The length of the polyQ domain determines the pathogenicity of the protein, with lengths of $<35$ being non-pathogenic and lengths of $35+$ being pathogenic.

Although the exact size depends on the length of the polyQ domain, full-length htt consists of $\sim 3142$ amino acids and has a molecular weight of approximately $348 \mathrm{kDa} .^{24}$ Until 
recently, it was not possible to express and purify the full-length protein, ${ }^{25}$ and as a result many studies were performed with biologically relevant N-terminal htt fragments. Here, the N-terminal fragment htt-exon1 was extensively used. Htt-exon1 has been identified in HD postmortem brains $^{26}$ and is a stable fragment that is constantly generated in the brain through proteolysis of full-length htt. ${ }^{27} \mathrm{Htt}$-exon1 is responsible for the accumulation and aggregation of htt in the striatum in $\mathrm{HD}^{28}$ and recently was identified as the most pathogenic naturally-occurring htt fragment. ${ }^{29}$ In addition, truncation of htt to htt-exon1 in HD140Q knock-in mice results in HDlike phenotypes and age-dependent accumulation similar to that observed with the full-length protein. ${ }^{28}$ While htt-exon1 is an important fragment in both mouse models and HD patients, it is also an ideal system for physiologically relevant in vitro biophysical studies as oligomers and fibrils formed by purified htt-exon 1 are similar morphologically to oligomers isolated from R6/2 and HD150Q knock-in mice. ${ }^{30}$ Htt-exon1 is comprised of three domains, one of which is the polyQ tract that begins at residue 18. The polyQ domain is flanked on the N-terminal side by 17 amino acids (Nt17) that play a role in initiating aggregation ${ }^{31-33}$ and lipid binding, ${ }^{34-36}$ and on the C-terminal side by a proline-rich domain containing two polyproline (polyP) stretches that slows htt nucleation ${ }^{37}$ and modulates htt-lipid interaction. ${ }^{34}$

\subsection{Huntingtin aggregation mechanisms and toxicity}

Once the polyQ domain of htt surpasses a threshold of 35 repeats, the free energy penalty of $\beta$-rich structure formation decreases, encouraging the formation of aggregate species. ${ }^{38}$ Pure polyQ peptides can misfold to form oligomers ${ }^{39}$ and amyloid-like fibrils ${ }^{40,41}$ on their own, and the incorporation of polyQ domains into a protein is enough to trigger polyQ-mediated aggregation. ${ }^{42}$ In fact, a number of diseases are caused by expanded polyQ domains in proteins, including Spinobulbar muscular atrophy, Machado-Joseph disease, and Huntington's Disease. ${ }^{43}$ 
The misfolding of polyQ-containing proteins, including mutant htt, into aggregate species is a complex process that occurs via multiple different pathways that are not mutually exclusive. Therefore, numerous mechanisms occur simultaneously, resulting in heterogeneous mixtures of aggregate species such as oligomers, fibrils, and inclusion bodies. ${ }^{31,44,45}$ While the specific aggregation details can vary by protein, proteins containing expanded polyQ tracts tend to follow the generic polyQ-mediated aggregation schemes. ${ }^{43}$ Several general mechanisms have been proposed for polyQ-containing protein aggregation, and polyQ-containing peptides sample multiple conformers that promote the progression through different aggregation pathways. Many of these mechanisms are referred to as "on-pathway", as they result in the formation of fibrils (Fig. 1.3). The process of polyQ amyloid fibril formation is initiated via a thermodynamically unfavorable rearrangement of a monomer into one of many non-native conformations. ${ }^{20}$ In nonnative conformations associated with aggregation, the polyQ region of the protein forms a monomeric or multimeric $\beta$-sheet through a combination of intramolecular and intermolecular interactions. The formation of this $\beta$-rich nucleus is referred to as the nucleation step and is the rate-limiting step in htt aggregation. ${ }^{46-48}$ In one widely accepted aggregation pathway for pure polyQ peptides, a monomer undergoes a conformational rearrangement to a structure that directly nucleates fibril formation..$^{40,41,48}$ The nucleus formation sets off a nucleation-dependent polymerization ${ }^{49}$ that results in the rapid expansion of fibrillar aggregates during what is referred to as the elongation phase. ${ }^{46,48}$ In another prominent on-pathway aggregation scheme, fibril formation occurs via oligomeric intermediate structures that undergo a slow structural rearrangement into a $\beta$-rich structure. ${ }^{50,51}$ While this aggregation scheme can occur with pure polyQ peptides, it occurs more prominently when the polyQ sequence is in the context of a larger protein like htt. ${ }^{52}$ The increased elongation of fibrils is also encouraged through the dissociation 
of oligomers into monomer species that can add to the exposed $\beta$-strands at the fibril ends. ${ }^{50,53}$ PolyQ-containing peptides can also sample conformations that promote the progression of offpathway aggregation pathways. ${ }^{54}$ Off-pathway aggregation mechanisms lead to the formation of a number of aggregates that do not go on to form fibrils, such as small annular aggregates, amorphous aggregates, and off-pathway oligomers. ${ }^{44}$ Monomers and higher order aggregate species can then accumulate into large aggregate species called inclusions that are a hallmark of HD. $^{23}$

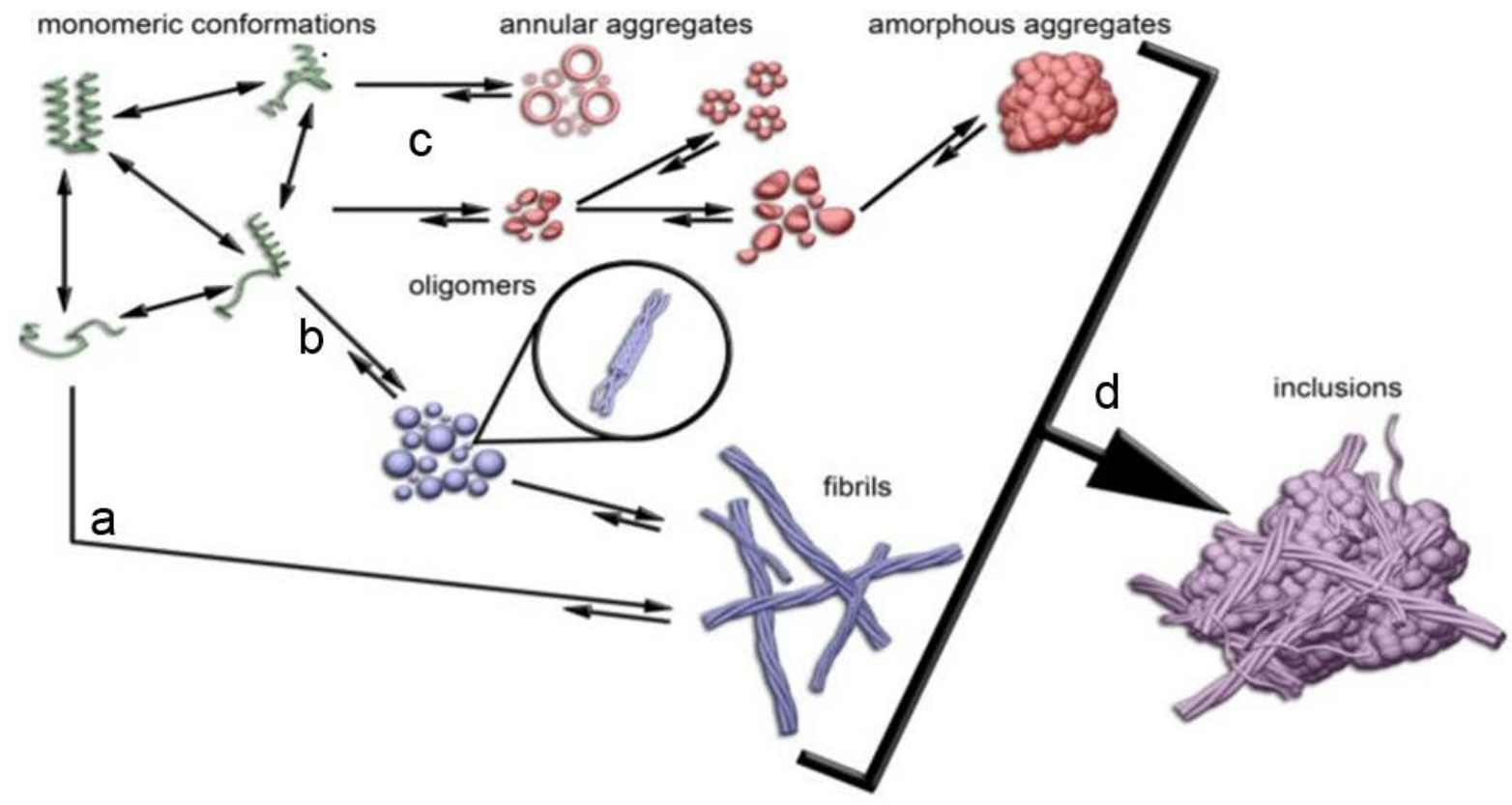

Figure 1.3 Htt aggregation pathways. Htt aggregates via three generally accepted aggregation mechanisms that all begin with the misfolding of a monomer into a non-native conformation. On-pathway aggregation, which results in $\beta$-rich fibrillar structures, can occur through (a) the direct misfolding of monomeric htt or through (b) oligomeric intermediate species. (c) Off-pathway aggregation results in the formation of oligomers, annular aggregates, and large amorphous aggregates. (d) Together, fibrils and amorphous aggregates can accumulate to form large inclusions that are observed in the brains of HD patients. Reprinted with permission from Adegbuyiro, A. et al. Proteins Containing Expanded Polyglutamine Tracts and Neurodegenerative Disease. Copyright 2017 American Chemical Society. 
The complexity of huntingtin aggregation can result in structural variation within subsets of aggregate species. ${ }^{33,55}$ Early monomeric conformational and structural variability results in the formation of oligomers with large degrees of structural variance, with some oligomers exhibiting $\beta$-cross interactions between monomers while others are non-amyloid $\alpha$-helix rich structures. ${ }^{50,56}$ In addition to varying in structure, the oligomers can also differ greatly in size; oligomeric htt species can range from small species like tetramers or dodecamers ${ }^{50,57}$ to large species containing upwards of hundreds of monomers. ${ }^{23,57}$ These populations are observed with purified proteins, as well as in the brains of R6/2 mice containing pathogenic-length htt-exon $1 .{ }^{30}$ With htt fibrils, despite a consistent $\beta$-hairpin-based polyglutamine core, ${ }^{53,58}$ structural characterization has revealed the potential for variation in structure and neurotoxicity as a result of the placement of the flanking domains. ${ }^{59,60}$ Variation in htt aggregate structure can result from numerous factors such as temperature ${ }^{61}$ polyglutamine length, ${ }^{23}$ and cellular environment. ${ }^{62-64}$

Due to the heterogeneous nature of htt aggregation, it can be difficult to distinguish which aggregate species are most involved in toxicity. There is much debate concerning the most toxic aggregate forms, as many distinct aggregate species have been linked to various toxic mechanisms. ${ }^{55,65,66}$ The diffusely distributed population of htt aggregates in cells has been identified as significantly toxic through cell survival analysis ${ }^{67,68}$ and is comprised of a variety of aggregate species ranging from small oligomers ${ }^{69-71}$ to amorphous aggregates and fibrils. ${ }^{72,73}$ Oligomers have been identified as a complex toxic population ${ }^{74}$ that induces ER stress in striatal neurons ${ }^{75}$ and can initiate htt aggregation in vitro. ${ }^{30}$ However, oligomers are not the only toxic species in the diffuse population, as there is also evidence that htt fibrils exhibit toxic properties. Htt-exon1 analogs that are mutationally constrained to favor the formation of fibrils over other aggregate species decrease the rate of survival in both cells and flies,${ }^{73}$ and homogenous 
populations of htt-exon1 fibrils can induce apoptotic cell death by binding to and permeabilizing lipid membranes. ${ }^{72}$ While some toxic mechanisms have been linked to specific aggregate species, the complexity of the diffuse population aggregate composition makes it challenging to identify the species responsible for much of htt cytotoxicity. Despite the involvement of many aggregate species in various toxic mechanisms, other aggregates may exhibit a protective effect. The formation of inclusion bodies reduces neuronal death in a potential neuroprotective mechanism, ${ }^{68,76}$ and exposure to conditions that allow aggregation but suppress inclusion formation results in increased huntingtin-induced cell death. ${ }^{74}$ The combination of htt aggregate cytotoxic and protective mechanisms highlights the complexity of mutant htt toxicity and HD pathogenesis.

\subsection{Nt17 structure and function}

The Nt17 domain is important in HD pathogenesis as it has been implicated as a promoter of htt aggregation. ${ }^{32,50,77} \mathrm{Nt} 17$ is intrinsically disordered, or virtually unstructured, in bulk solution, but undergoes a conformational shift to an amphipathic $\alpha$-helix in the presence of binding partners (Fig. 1.4) ${ }^{78,79}$ Briefly, an amphipathic $\alpha$-helix consists of separate predominately hydrophobic and predominately hydrophilic sides. This conformational shift has been implicated as an important driver of htt aggregation, as Nt17 $\alpha$-helices can associate with each other to form varying multimeric species ranging from compact globular structures to elongated helical bundles. ${ }^{31}$ The variation in multimer structures exposes different residues of $\mathrm{Nt17}$ to the solvent, resulting in changes to downstream aggregation and potentially Nt17-driven htt oligomerization. ${ }^{31}$ One of these experimentally observed multimeric structures is an $\alpha$-helical tetramer structure ${ }^{31,50}$ that builds up into higher order, $\alpha$-helix rich oligomers, making these tetrameric Nt17 species crucial in the early stages of aggregation. ${ }^{32,33}$ Oligomers are a potent 
toxic aggregate species of htt and Nt17 is crucial for the formation of oligomers, as removing Nt17 from htt-exon1 completely eliminates the formation of nonamyloid oligomers. ${ }^{77,80} \mathrm{In}$ addition to encouraging oligomer formation, Nt17 plays a role in fibrillar aggregate formation; the $\alpha$-helical oligomers formed as a direct result of $\mathrm{Nt} 17$ association can undergo a slow structural transition to $\beta$-sheet aggregates, eventually resulting in fibril formation. ${ }^{50}$

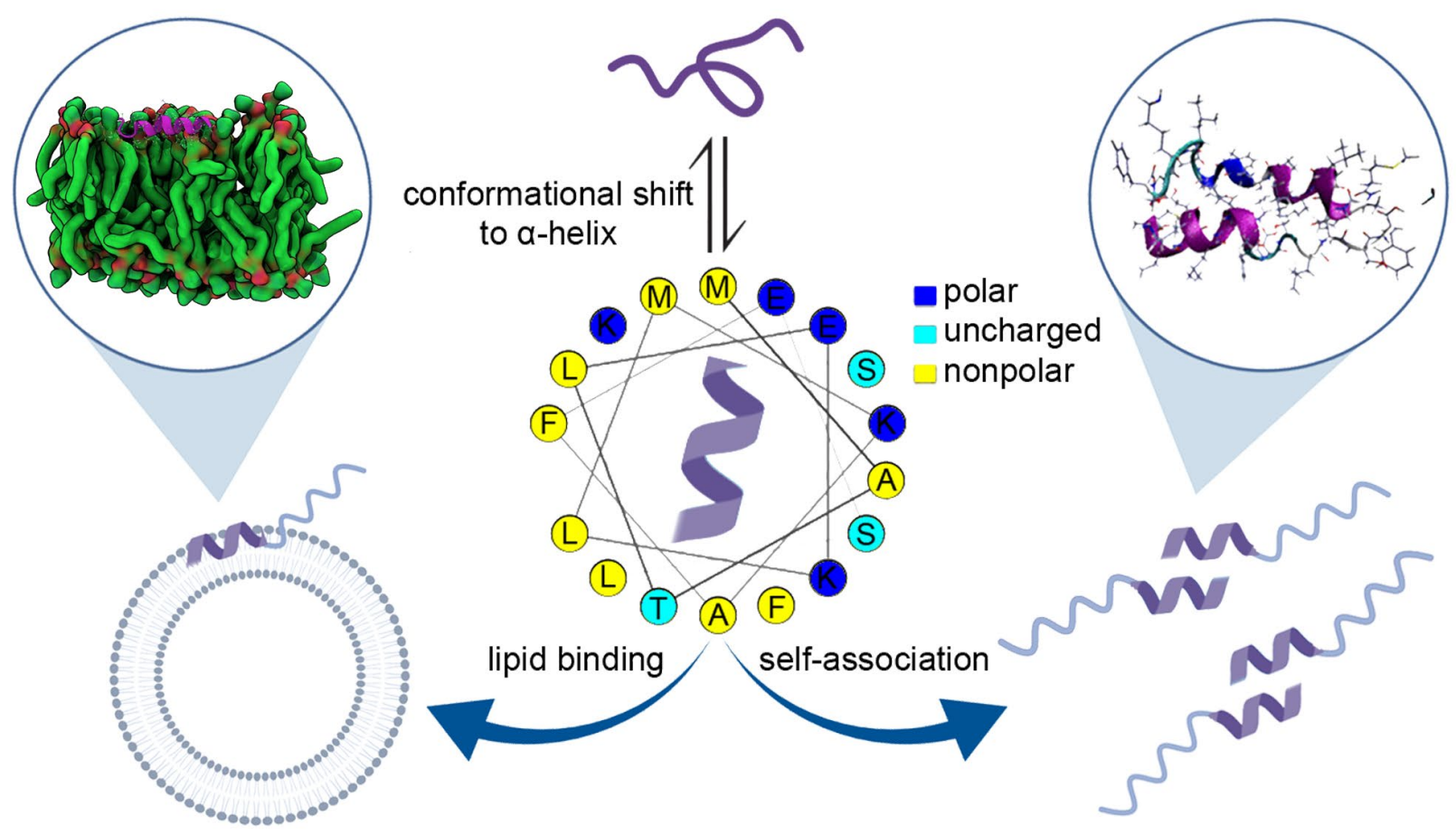

Figure 1.4 The structure and function of the $\mathbf{N t 1 7}$ domain. ${ }^{157} \mathrm{Nt} 17$ is unstructured in bulk solution but undergoes a conformational shift to an amphipathic $\alpha$-helix with one hydrophobic and one hydrophilic face in the presence of binding partners. The $\alpha$-helix can be involved in lipid binding through the insertion of the helix into the membrane or in the early stages of aggregation through self-association. Adapted (in part) with permission from Arndt, J. R. et al. Nucleation Inhibition of Huntingtin Protein (htt) by Polyproline PPII Helices: A Potential Interaction with the N-Terminal $\alpha$-Helical Region of Htt. Copyright 2019 American Chemical Society.

In addition to spearheading htt aggregation, the Nt17 amphipathic $\alpha$-helical structure acts as a lipid binding domain (Fig. 1.4). Nt17 exhibits many properties associated with the amphipathic $\alpha$-helical motif, such as an association with lipid binding ${ }^{81,82}$ and the preferential 
binding of defects induced by curvature. ${ }^{83}$ As a lipid binding domain, Nt17 is crucial for the interaction of htt with lipid membranes; polyQ peptides alone do not appreciably insert into lipid membranes, but polyQ peptides flanked by Nt17 directly interact with and disrupt lipid vesicles. ${ }^{34}$ In addition, Nt17 preferentially facilitates the interaction of htt-exon1 peptides with supported lipid bilayers with higher degrees of curvature ${ }^{84}$ The interaction of Nt17 with lipid bilayers involves the insertion of nonpolar residues into the membrane through four general steps: approach, reorganization, anchoring, and insertion. ${ }^{35}$ The approach is driven by long-range electrostatic interactions between the peptide and the lipid headgroups, followed by the reorganization where the peptide undergoes conformational rearrangements to bring the nonpolar peptide residues closer to the hydrophobic membrane core. The anchor step is initialized when one of those nonpolar restudies positions inside the membrane core, and then additional nonpolar residues partition into the membrane during the insertion step as the peptide undergoes further structural rearrangement. The end result is a stable $\alpha$-helical structure that is close to parallel with the membrane surface and buries the nonpolar residues of Nt17 in the hydrophobic membrane core..$^{35,36,78}$

Nt17 has numerous sites that can undergo post-translational modifications (PTM) like acetylation, ${ }^{85}$ SUMOylation, ${ }^{86,87}$ phosphorylation, ${ }^{88,89}$ and oxidation. ${ }^{90}$ Modification of Nt17 directly influences htt function and HD progression by impacting htt membrane association, ${ }^{64,88,91}$ translocation, ${ }^{92,93}$ aggregation, ${ }^{64,88,91,94}$ and toxicity. ${ }^{86,94,95}$ The impact of Nt17 PTM on htt aggregation and lipid binding have revealed the crucial role specific amino acids play in these processes. The acetylation of the three lysine residues $(\mathrm{K} 6, \mathrm{~K} 9, \mathrm{~K} 15)$ using a selective covalent label decreases the extent of htt lipid binding and membrane disruption, while also decreasing fibril formation in favor of large, globular aggregate species.$^{64}$ These lysine 
residues can also be SUMOylated; SUMOylation of K6, K9, and K15 prevent htt accumulation on lipid bilayers and promote the formation of large, amorphous aggregate species while reducing the overall extent of aggregation. ${ }^{86,91}$ The lysine residues that lie at the edge of the hydrophilic region of the amphipathic $\alpha$-helix (K6 and K15) are protected in early htt aggregate species, indicating a potential role of the residues in the early stages of $\mathrm{Nt} 17$ self-association. ${ }^{96}$ Nt17 can also undergo phosphorylation at three residues (T3, S13, S16), resulting in changes to htt aggregation and lipid binding. Phosphorylation at residue S13 or S16 results in significant decrease in Nt17 helical content, resulting in decreased membrane binding and lowered rate and extent of aggregate formation. ${ }^{88,97}$ On the other hand, T3 phosphorylation modulates mutant htt conformation by stabilizing the Nt17 amphipathic $\alpha$-helix, but still results in a significant inhibition of htt aggregation. ${ }^{98,99}$ Similarly, oxidation of M7 increases the stability of the $\alpha$-helix, expanding it by 2 residues, but reduces the binding affinity of htt for micelles $\sim 3-4$ fold. ${ }^{90}$ Together, the results obtained from PTM studies on htt reveal the specific amino acids that may play a critical role in lipid binding and the early stages of aggregation.

\subsection{Functions and toxic events associated with htt-lipid interaction}

Interaction between htt and lipid membranes has been associated with a wide range of normal htt functions. Vesicular and mitochondrial trafficking are impaired in htt knock-out mice, implicating htt as an important contributor in axonal transport. ${ }^{100,101} \mathrm{Htt}$ is also involved in trafficking between the nucleus and cytoplasm through Nt17-mediated reversible association with the endoplasmic reticulum (ER), endosomes, and autophagic vesicles. ${ }^{102}$ Membrane association is also crucial for htt localization to subcellular components like the nucleus and plasma membrane. ${ }^{103,104} \mathrm{Htt}$ is detected in synaptosomal membrane fractions and has been observed by immunoelectron microscopy to localize to vesicle membranes and microtubules. ${ }^{105-}$ 
107 There is increasing evidence that a component of HD pathology is the result of toxic loss-offunction, potentially due to the disruption of interaction between htt and membranes. Htt knockout mice exhibit increased apoptosis and early embryonic lethality, ${ }^{108,109}$ and even mice that are heterozygous for htt knockout exhibit significant neuronal degeneration, ${ }^{108,110}$ indicating that $h t t$ is an essential protein. Direct disruption of htt functions related to membrane association have detrimental effects such as organelle dysfunction, ${ }^{111,112}$ cell death, ${ }^{113}$ and brain degeneration. ${ }^{100,101}$

While the interaction between htt and lipid membranes is important for proper function, these interacts change when htt has an expanded, pathogenic-length polyQ domain. ${ }^{114,115}$ There is a polyQ length dependence on the formation and stability of the Nt17 amphipathic $\alpha$-helix, ${ }^{50}$ and htt-exon1 disrupts the structural integrity of lipid bilayers in a polyQ-dependent manner. ${ }^{114,116}$ As longer polyQ domains also result in quicker aggregation, ${ }^{20,48,117}$ it has been hypothesized that the polyQ-length dependence on htt-lipid interaction is in part a result of this fast aggregate formation. ${ }^{116}$ In fact, numerous aggregate species have been implicated in htt membrane interaction and disruption. ${ }^{54,118-122}$ Oligomeric htt-exon1 binds to and disrupts membranes consisting of brain lipids, resulting in membrane leakiness and toxicity in neurons. ${ }^{118}$ Fibrillar aggregates interact with ER membranes to distort morphology and dynamics, ${ }^{119}$ while membranous structures are incorporated into htt inclusions. ${ }^{121,122}$ With many of htt normal functions involving direct interaction with lipid membranes, the increased association and detrimental effects of $\mathrm{htt}$ aggregates on lipid membranes may play a critical role in HD pathology. 
a

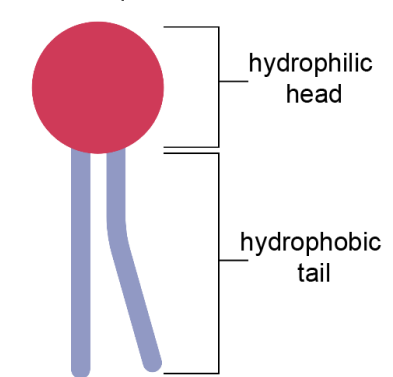

C

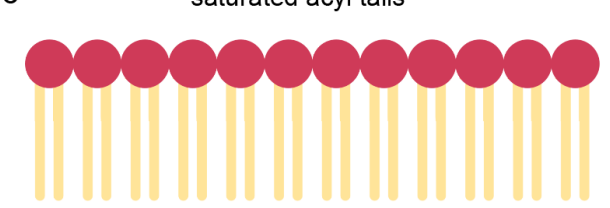

monounsaturated acvl tails

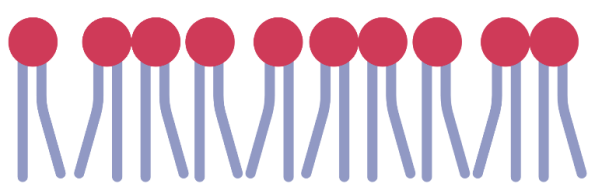

b

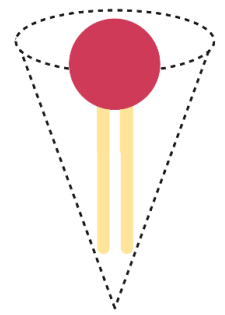

lipid shape
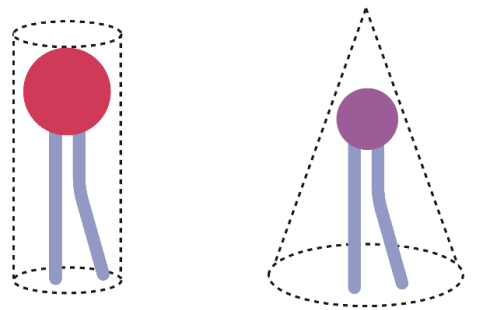

d effect of sterols on membrane packing

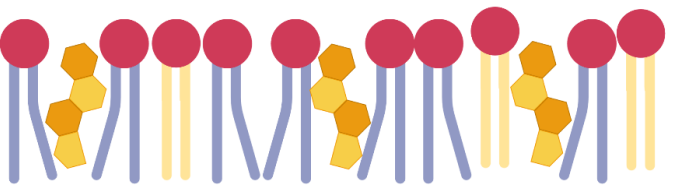

e

Figure 1.5 Factors that influence lipid membrane properties. ${ }^{157}$ (a) Lipids consist of a hydrophilic head and hydrophilic tail. (b) The size of the lipid head and saturation of the tail alter the shape of a lipid monomer. (c) Lipid tail saturation alters how tightly lipid monomers can pack together in a membrane. (d) Additional membrane components, such as sterols, can influence the packing and properties of lipid membranes. (e) Membrane curvature increases the propensity and size of available membrane effects.

In addition to being influenced by properties of the protein - such as aggregation state the interaction between htt and lipids is also influenced by lipid properties. Lipids are comprised of a hydrophilic head and hydrophobic tail (Fig. 1.5a), so they spontaneously form bilayers in aqueous environments to shield the tails from water. The size and charge of the headgroup, as well as the length and saturation of the lipid tail, play a role in determining the lipid shape, how the lipid monomers assemble, and the properties of the lipid bilayer (Fig. $1.5 b)$. For example, the size of the lipid headgroup plays a role in the preferred curvature of a bilayer system, ${ }^{123}$ and the size and saturation of the tail influence properties such as packing and 
fluidity. ${ }^{124}$ Double bonds in the lipid tail provide "kinks" that make it more difficult for the lipids to pack together, resulting in increased fluidity with increased degrees of tail unsaturation (Fig. 1.5c). Increased membrane fluidity results in a higher propensity for the occurrence of membrane defects, ${ }^{125}$ which is important for the Nt17 defect-detecting amphipathic $\alpha$-helix. ${ }^{126}$ The physicochemical properties of a membrane system are largely determined by the membrane composition (Fig. 1.5d), ${ }^{127}$ which is likely why alterations to membrane composition influence the interaction between htt and lipid bilayers. The addition of cholesterol to total brain lipid extract (TBLE) decreases htt insertion and htt-induced permeabilization of vesicles. ${ }^{62}$ Increased concentrations of sphingomyelin (SM) or ganglioside (GM1) in TBLE also decreases htt-lipid interaction, but increased SM content results in a higher susceptibility to htt-induced permeability while GM1 has no effect. ${ }^{128}$ In addition to lipid shape and composition, the propensity and size of bilayer defects are largely influenced by membrane curvature (Fig. 1.5e). ${ }^{123,129} \mathrm{Nt} 17$ preferentially associates with curved membranes over flatter membranes, ${ }^{84}$ likely due to the increased likelihood of membrane defects when curvature is induced. ${ }^{123,129}$ The interaction of htt with lipid membranes is a complex mechanism that is influenced by a wide array of lipid properties, and understanding the effect of these properties on htt membrane selectivity is important in understanding the normal function and cytotoxic effects of mutant htt.

\subsection{The influence of membranes on huntingtin aggregation}

Because of the dual roles of the Nt17 amphipathic $\alpha$-helix in lipid binding and the early stages of aggregation, the presence of membranes as a potential binding partner could influence htt aggregation. In computational studies, htt monomers are capable of perturbing the physical properties of POPC bilayers in a manner that favors dimerization and the early stages of aggregation. ${ }^{36}$ This may explain why surfaces, such as membranes, promote specific aggregation 
pathways. ${ }^{130}$ Biophysically, POPC/PS lipid vesicles have been found to catalyze the aggregation of htt-exon1(46Q) by anchoring to the membrane and increasing the local protein concentration, ${ }^{131}$ but this effect is dependent on peptide:lipid ratios and the polyQ length. ${ }^{132}$ On the other hand, the presence of vesicles comprised of total brain lipid extract (TBLE) decreases the fibrillization of the same htt fragment. ${ }^{63,128}$ This indicates that composition is likely a factor in the effect of a membrane on htt aggregation, a notion that is supported by the fact that DOPCSM-Chol vesicles have no effect on aggregation ${ }^{118}$ but spikes of SM in TBLE systems decreases oligomer formation ${ }^{128}$ and cholesterol decreases htt fibrillization as a function of concentration. ${ }^{62}$ In addition, the presence of lipid membranes can have a stabilizing effect on certain aggregate species like oligomers. ${ }^{34}$ Lipids are also incorporated into or associated with a large variety of htt aggregates. Htt co-aggregates with neuronal lipids in transgenic mouse models, ${ }^{120}$ and htt inclusion bodies incorporate multi-vesicular membranes and mitochondria into their surfaces. ${ }^{122}$ The association of htt with brain lipid LUVs alters the nucleation phase of aggregation, ${ }^{118}$ potentially enhancing the formation, stabilization, and morphology of toxic aggregate species that can incorporate lipids and may represent an important factor in HD progression.

\subsection{Combination of experimental approaches to study huntingtin at different scales}

Huntingtin aggregation is a complex process that begins with the rearrangement of mutant htt into a non-native conformation ${ }^{20}$ and results in the formation of large aggregate species. ${ }^{23,43,66}$ Alterations to monomeric htt, like through a PTM, influence the aggregation and lipid binding of htt likely by influencing the early stages of interaction. ${ }^{64,91}$ A particularly challenging component of studying htt interaction with binding partners is finding techniques that have the resolution to study the early mechanisms and structures as well as the resulting down-stream aggregate morphologies and populations. To overcome this issue, studies presented 
in Chapter 4 and Chapter 5 will combine native mass spectrometry and atomic force microscopy to study how early Nt17-lipid interactions effect downstream aggregation.

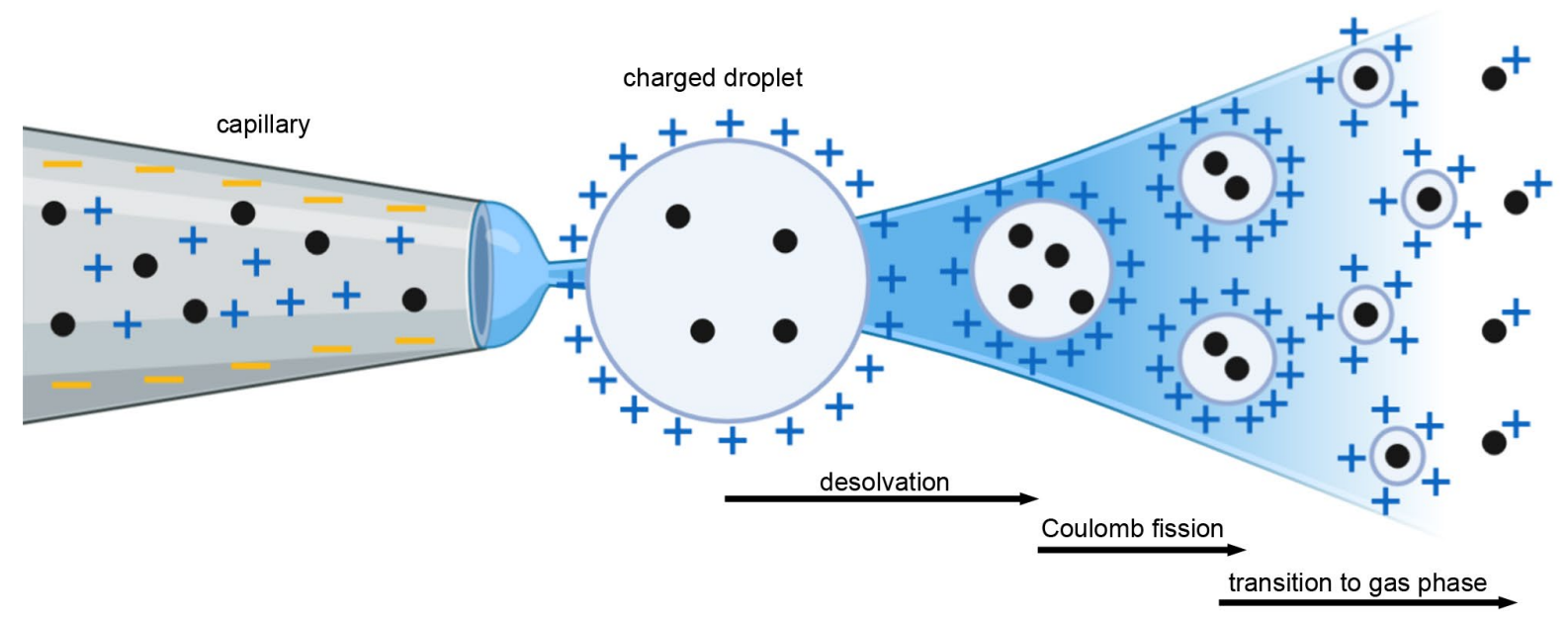

Figure 1.6 Electrospray ionization produces gas-phase ions from solution. ${ }^{157}$ For electrospray ionization, charge is applied to a solution of analyte in the capillary tip, forming charged droplets. The droplets undergo solvent evaporation, decreasing the size of the droplet until the repulsion of the charges in the droplet results in Coulomb fission. The processes of desolvation and Coulomb fission are repeated until a single charge analyte ion transitions to the gas phase.

Mass spectrometry has recently been established as a useful technique to study the early phases of htt interactions, from Nt17 monomeric and multimeric structures ${ }^{31,96}$ to the beginning stages of htt lipid binding. ${ }^{133}$ Many of these studies utilize electrospray ionization (ESI), a technique that converts solution-phase analytes into gas-phase ions (Fig. 1.6) ${ }^{134,135}$ In order to accomplish this, charged droplets are produced in the electrospray capillary tip, and then the charged particles are shrunken via solvent evaporation. Eventually, the electrostatic repulsion of the charges in the shrinking droplet overcome the surface tension holding the droplet together and the droplet undergoes Coulomb fission. ${ }^{136}$ The droplets undergo desolvation and Coulomb fission until the droplets contain approximately one analyte ion. ${ }^{137}$ This is considered a "soft" 
ionization technique because non-covalent interactions, native state, and complexes are largely preserved without fragmentation. ${ }^{138-140}$ When the biological analytes are prepared in a nondenaturing solvent and sprayed with ESI, the technique is called native mass spectrometry (Native MS). Because samples can be prepped in physiologically relevant solvents and undergo ionization without fragmentation or disruption, native MS is perfect to study the early stages of htt fragments with binding partners such as other htt fragments, small molecules, or lipid membranes.

To determine the effects that these early stage interactions have on htt aggregation, atomic force microscopy (AFM) can be used to study the later stage aggregates (Fig. 1.7). AFM was developed as a method to perform three-dimensional imaging of surfaces, ${ }^{141}$ making it perfect for measuring the structural and morphological features of protein aggregates. While electron microscopy (EM) is often used to study amyloid aggregates due to higher scan speeds, ${ }^{142}$ it also requires extensive sample preparation that can distort aggregate morphology. ${ }^{143-145}$ Also, EM images are 2D, while AFM provides the additional advantage of a z-dimensional measurement to provide 3D surface images. ${ }^{146}$ Due to its nanoscale resolution and ability to be operated in both air and solution, AFM has emerged as a tool to study nanostructure formation, ${ }^{130,147}$ morphological characteristics of protein aggregates, ${ }^{64,91,148,149}$ and the mechanical changes in lipid bilayers as a result of interaction with amyloid proteins. ${ }^{150-152}$ In general, an AFM images a surface by measuring the vertical displacement of a probe upon interaction with the sample surface. The probe - an ultrasharp tip, in this case - is attached to the end of a cantilever and experiences forces from the sample as it is brought close to the surface. The cantilever acts as a harmonic oscillator as the tip approaches the surface and experiences a measurable deflection according to Hooke's Law as a result of forces between the tip and sample. A laser aligned 
to reflect off the back of the cantilever deflects as a result of the vertical displacement, which changes the reflected laser position on a photodiode detector. The displacement measured by the photodiode detector is corrected via the feedback loop, where the piezo adjusts the vertical position of the sample stage as necessary to maintain a constant cantilever deflection or amplitude. This process occurs for each pixel of the desired surface area, and the piezo scanner moves the cantilever back and forth across the surface sample to create a complete 3D image.

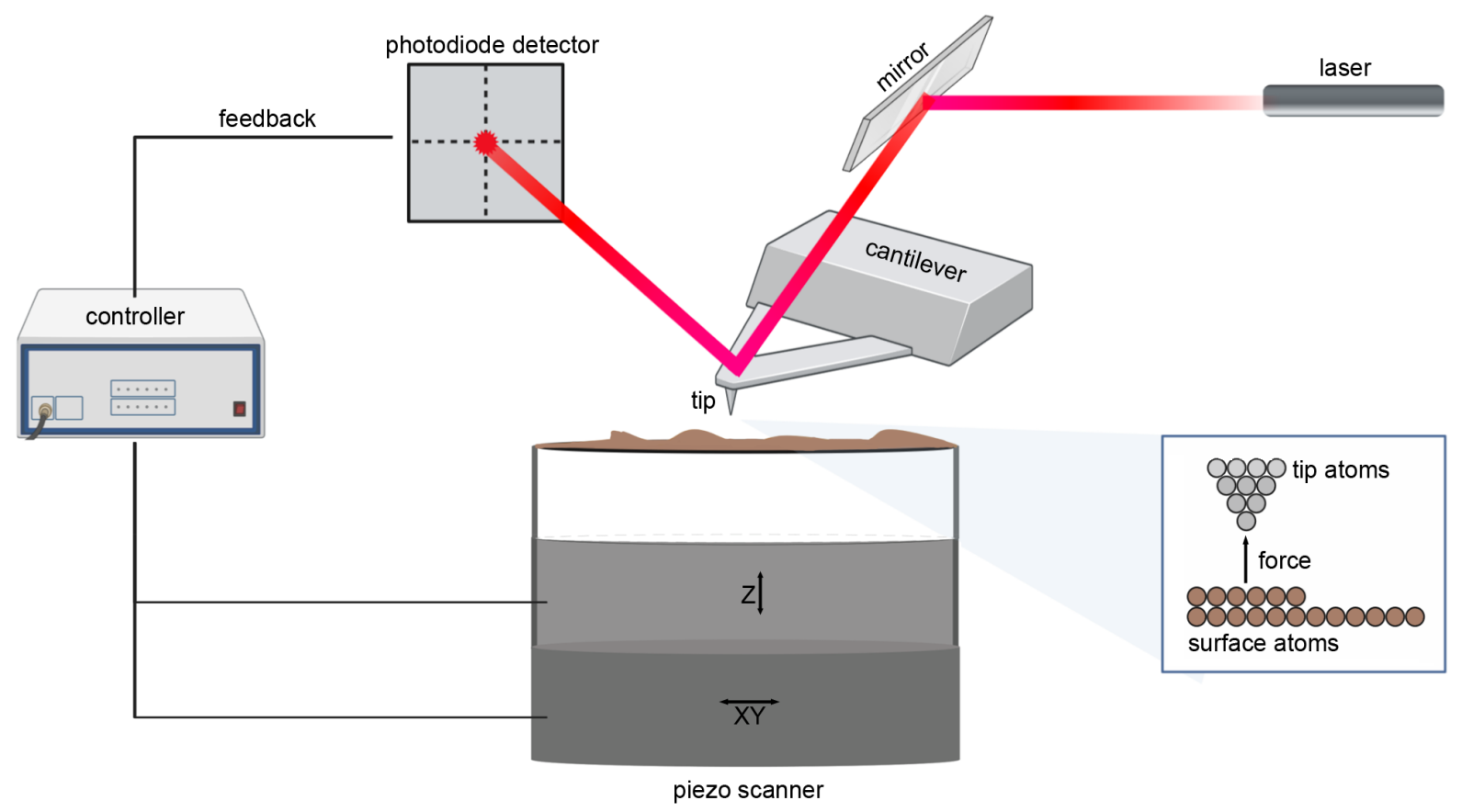

Figure 1.7 Schematic representation of an AFM. ${ }^{157}$ An ultra-sharp tip is brought in close contact with the sample surface, resulting in a measurable deflection of the cantilever. The deflection is determined by a laser reflected off the back of the cantilever onto a photodiode detector. The photodiode provides information about the deflection to the feedback loop that results in the production of a three-dimensional image of the artifact measured on the surface.

While many modes of AFM have been developed for special purposes, the most commonly utilized AFM techniques are contact mode, non-contact mode, and tapping mode. In contact mode, the probe is brought in direct contact with the surface and the deflection of the 
cantilever is measured. A voltage is applied to the piezo to raise or lower the sample and restore the desired deflection. The voltage required for the correction provides a measure of the height of the surface feature. Contact mode has many advantages, but it is not ideal for imaging soft biological samples because the intense frictional and adhesive forces can result in damage of samples and distorted image data even at the lowest tracking forces available. ${ }^{153,154}$ In noncontact AFM, the probe never contacts the surface; instead, the forces applied on the probe from the sample as the tip approaches the surface result in a change in the amplitude of the cantilever's resonant vibration. Here, the force interactions are measured by the change in amplitude at a constant frequency or by measuring the change in resonant frequency. ${ }^{141,155}$ However, noncontact mode presents limitations such as slower scan speeds and high potential for unstable feedback. ${ }^{156}$ Because of the disadvantages of contact and non-contact modes, the studies presented here will utilize tapping mode AFM. In tapping mode, the cantilever is oscillated near its resonance frequency and placed close enough to the surface that during each oscillation it briefly makes contact with the surface. When the tip intermittently contacts the surface, the amplitude of the cantilever oscillation changes as a result of surface characteristics. For example, the cantilever has less room to oscillate when going over a bump, thus resulting in a decrease in oscillation amplitude. Through the feedback loop, the piezo scanner raises or lowers the sample stage to maintain a constant cantilever amplitude and the amount the sample stage must move determines the height of the surface features. Tapping mode is ideal for studying amyloid aggregate species because it results in lower forces and less damage to soft biological samples while maintaining a high lateral resolution. ${ }^{156}$ 


\subsection{Dissertation Rationale}

The association of htt with lipid membranes is crucial for normal htt function but can also induce toxic effects when the htt has a pathogenic length polyQ domain. The toxic effects of mutant htt on lipid membranes result in organelle dysfunction and neuronal degeneration as HD progresses, and yet the properties of the lipid membrane that influence mutant htt membrane selectivity are widely unknown. In addition, despite the established fact that lipid membranes alter htt aggregation, whether this effect is consistent with membranes of different compositions has not been studied. Here, we explore the effect that membrane physicochemical properties have on htt aggregation, lipid binding, and its interaction with potential therapeutic agents.

In Chapter 2, we present a method of normalizing polydiacetylene (PDA) lipid binding assays to allow for the direct comparison of interaction with different lipid systems. The sensitivity of PDA/lipid vesicles comprised of DOPC, POPC, POPE, or POPG were examined, allowing us to compare systems with different fluidity, headgroup size, charge, and tail saturation. The normalization protocol reduced batch-to-batch and lipid-to-lipid PDA variability to establish a cost-effective, quick, and reproducible method of measuring membrane affinity. Finally, the results of the PDA assays after normalization were verified with small molecule and peptide applications through comparison to previous studies. By utilizing this normalization method, we can further study the interaction between mutant htt and different lipid membranes,

In Chapter 3, we determined how lipid membranes of different composition influenced the effectiveness of two known amyloid aggregation inhibitors, epigallocatechin 3-gallate (EGCG) and curcumin, in inhibiting htt fibrillization. Htt aggregation in the presence and absence of the small molecules and lipid (POPC or TBLE) were monitored over time using 
thioflavin $\mathrm{T}$ aggregation assays (ThT), revealing that the effectiveness of curcumin in particular is largely decreased by the presence and composition of the lipid membranes. Finally, PDA assays were utilized to determine how the interaction of htt with pure or physiological lipid systems was influenced by the presence of each small molecule. This chapter reveals that htt aggregation is a complex mechanism that is significantly altered by cellular components such as lipid membranes, and that the effect is dependent on the composition of the membrane.

Once Chapter 3 results indicated that htt association with the lipid systems varied with composition, we examined the role that lipid physicochemical properties have in these interactions. In order to do this, pure lipid systems were selected that have the same tail group and different head (Chapter 4) or same head group and different tail (Chapter 5). The extent and rate of htt formation in the presence of each lipid system was monitored with ThT assays, and the aggregate morphologies were observed using atomic force microscopy (AFM). In order to determine why the lipids have different effects on htt aggregation, PDA assays were performed with each lipid system and Nt17-lipid complexes were studied with native mass spectrometry. For additional mechanistic analysis in Chapter 5, the early stages of Nt17-lipid association were examined with weighted ensemble molecular dynamics simulations. Chapters 4 and 5 sought to determine lipid properties - such as fluidity, packing, and charge - that influence htt-lipid interaction with the hopes of explaining the selective association of htt to specific membranes.

\subsection{References}

1. Roos, R. A. Huntington's disease: a clinical review. Orphanet J. Rare Dis. 5, 40 (2010).

2. Chung, J. A. \& Cummings, J. L. NEUROBEHAVIORAL AND NEUROPSYCHIATRIC SYMPTOMS IN ALZHEIMER'S DISEASE: Characteristics and Treatment. Neurol. Clin. 18, 829-846 (2000). 
3. Sveinbjornsdottir, S. The clinical symptoms of Parkinson's disease. J. Neurochem. 139, 318324 (2016).

4. Martin, J. B. Molecular basis of the neurodegenerative disorders. N. Engl. J. Med. 340, 19701980 (1999).

5. Carrell, R. W. \& Lomas, D. A. Conformational disease. The Lancet 350, 134-138 (1997).

6. Dobson, C. M. Protein misfolding, evolution and disease. Trends Biochem. Sci. 24, 329-332 (1999).

7. Soto, C. Unfolding the role of protein misfolding in neurodegenerative diseases. Nat. Rev. Neurosci. 4, 49-60 (2003).

8. Wolynes, P. G., Onuchic, J. N. \& Thirumalai, D. Navigating the folding routes. Science 267, 1619-1620 (1995).

9. Dill, K. A. \& Chan, H. S. From Levinthal to pathways to funnels. Nat. Struct. Biol. 4, 10-19 (1997).

10. Dinner, A. R., Sali, A., Smith, L. J., Dobson, C. M. \& Karplus, M. Understanding protein folding via free-energy surfaces from theory and experiment. Trends Biochem. Sci. 25, 331339 (2000).

11. Anfinsen, C. B. The formation and stabilization of protein structure. Biochem. J. 128, 737749 (1972).

12. Vendruscolo, M., Paci, E., Karplus, M. \& Dobson, C. M. Structures and relative free energies of partially folded states of proteins. Proc. Natl. Acad. Sci. U.S.A. 100, 14817-14821 (2003).

13. Jahn, T. R. \& Radford, S. E. Folding versus aggregation: Polypeptide conformations on competing pathways. Arch. Biochem. Biophys. 469, 100-117 (2008).

14. Reynaud, E. Protein Misfolding and Degenerative Diseases. in Nature Education vol. 9 (2010).

15. Wetzel, R. Kinetics and Thermodynamics of Amyloid Fibril Assembly. Acc. Chem. Res. 39, 671-679 (2006).

16. Finkel, T. Radical medicine: treating ageing to cure disease. Nat. Rev. Mol. Cell Biol. 6, 971976 (2005).

17. Huntington's disease - Symptoms and causes. Mayo Clinic https:/www.mayoclinic.org/diseases-conditions/huntingtons-disease/symptoms-causes/syc20356117. 
18. Penney, J. B., Vonsattel, J. P., MacDonald, M. E., Gusella, J. F. \& Myers, R. H. CAG repeat number governs the development rate of pathology in Huntington's disease. Ann. Neurol. 41, 689-692 (1997).

19. Snell, R. G. et al. Relationship between trinucleotide repeat expansion and phenotypic variation in Huntington's disease. Nat. Genet. 4, 393-397 (1993).

20. Bhattacharyya, A. M., Thakur, A. K. \& Wetzel, R. Polyglutamine aggregation nucleation: Thermodynamics of a highly unfavorable protein folding reaction. Proc. Natl. Acad. Sci. U.S.A. 102, 15400-15405 (2005).

21. Chen, S., Ferrone, F. A. \& Wetzel, R. Huntington's disease age-of-onset linked to polyglutamine aggregation nucleation. Proc. Natl. Acad. Sci. U.S.A. 99, 11884-11889 (2002).

22. Project*, T. U. S.-V. C. R. \& Wexler, N. S. Venezuelan kindreds reveal that genetic and environmental factors modulate Huntington's disease age of onset. Proc. Natl. Acad. Sci. U.S.A. 101, 3498-3503 (2004).

23. Legleiter, J. et al. Mutant Huntingtin Fragments Form Oligomers in a Polyglutamine Lengthdependent Manner in Vitro and in Vivo. J. Biol. Chem. 285, 14777-14790 (2010).

24. UPI000013D567. https://www.uniprot.org/uniparc/UPI000013D567?direct=yes\&sort=score.

25. Li, W., Serpell, L. C., Carter, W. J., Rubinsztein, D. C. \& Huntington, J. A. Expression and Characterization of Full-length Human Huntingtin, an Elongated HEAT Repeat Protein. $J$. Biol. Chem. 281, 15916-15922 (2006).

26. DiFiglia, M. et al. Aggregation of huntingtin in neuronal intranuclear inclusions and dystrophic neurites in brain. Science 277, 1990-1993 (1997).

27. Landles, C. et al. Proteolysis of mutant huntingtin produces an exon 1 fragment that accumulates as an aggregated protein in neuronal nuclei in Huntington disease. J. Biol. Chem. 285, 8808-8823 (2010).

28. Yang, H. et al. Truncation of mutant huntingtin in knock-in mice demonstrates exon1 huntingtin is a key pathogenic form. Nat. Commun. 11, 2582 (2020).

29. Barbaro, B. A. et al. Comparative study of naturally occurring huntingtin fragments in Drosophila points to exon 1 as the most pathogenic species in Huntington's disease. Hum. Mol. Genet. 24, 913-925 (2015).

30. Sathasivam, K. et al. Identical oligomeric and fibrillar structures captured from the brains of R6/2 and knock-in mouse models of Huntington's disease. Hum. Mol. Genet. 19, 65-78 (2010).

31. Arndt, J. R. et al. Huntingtin N-terminal monomeric and multimeric structures destabilized by covalent modification of heteroatomic residues. Biochemistry 54, 4285-4296 (2015). 
32. Williamson, T. E., Vitalis, A., Crick, S. L. \& Pappu, R. V. Modulation of polyglutamine conformations and dimer formation by the N-terminus of Huntingtin. J. Mol. Biol. 396, 12951309 (2010).

33. Vitalis, A. \& Pappu, R. V. Assessing the contribution of heterogeneous distributions of oligomers to aggregation mechanisms of polyglutamine peptides. Biophys. Chem. 159, 14-23 (2011).

34. Burke, K. A., Kauffman, K. J., Umbaugh, C. S., Frey, S. L. \& Legleiter, J. The Interaction of Polyglutamine Peptides with Lipid Membranes Is Regulated by Flanking Sequences Associated with Huntingtin. J. Biol. Chem. 288, 14993-15005 (2013).

35. Côté, S., Wei, G. \& Mousseau, N. Atomistic mechanisms of huntingtin N-terminal fragment insertion on a phospholipid bilayer revealed by molecular dynamics simulations. Proteins $\mathbf{8 2}$, 1409-1427 (2014).

36. Côté, S., Binette, V., Salnikov, E. S., Bechinger, B. \& Mousseau, N. Probing the Huntingtin 1-17 Membrane Anchor on a Phospholipid Bilayer by Using All-Atom Simulations. Biophys. $J$. 108, 1187-1198 (2015).

37. Arndt, J. R. et al. Nucleation Inhibition of Huntingtin Protein (htt) by Polyproline PPII Helices: A Potential Interaction with the N-Terminal $\alpha$-Helical Region of Htt. Biochemistry (2019) doi:10.1021/acs.biochem.9b00689.

38. Chen, M. \& Wolynes, P. G. Aggregation landscapes of Huntingtin exon 1 protein fragments and the critical repeat length for the onset of Huntington's disease. Proc. Natl. Acad. Sci. U.S.A. 114, 4406-4411 (2017).

39. Perutz, M. F., Johnson, T., Suzuki, M. \& Finch, J. T. Glutamine repeats as polar zippers: their possible role in inherited neurodegenerative diseases. Proc. Natl. Acad. Sci. U.S.A. 91, 5355-5358 (1994).

40. Chen, S., Berthelier, V., Hamilton, J. B., O’Nuallain, B. \& Wetzel, R. Amyloid-like features of polyglutamine aggregates and their assembly kinetics. Biochemistry 41, 7391-7399 (2002).

41. Chen, S., Berthelier, V., Yang, W. \& Wetzel, R. Polyglutamine aggregation behavior in vitro supports a recruitment mechanism of cytotoxicity11Edited by F. Cohen. J. Mol. Biol. 311, 173-182 (2001).

42. Stott, K., Blackburn, J. M., Butler, P. J. \& Perutz, M. Incorporation of glutamine repeats makes protein oligomerize: implications for neurodegenerative diseases. Proc. Natl. Acad. Sci. U.S.A. 92, 6509-6513 (1995).

43. Adegbuyiro, A., Sedighi, F., Pilkington, A. W., Groover, S. \& Legleiter, J. Proteins Containing Expanded Polyglutamine Tracts and Neurodegenerative Disease. Biochemistry 56, 1199-1217 (2017). 
44. Burke, K. A., Godbey, J. \& Legleiter, J. Assessing mutant huntingtin fragment and polyglutamine aggregation by atomic force microscopy. Methods 53, 275-284 (2011).

45. Counterman, A. E., Hilderbrand, A. E., Barnes, C. A. S. \& Clemmer, D. E. Formation of peptide aggregates during ESI: Size, charge, composition, and contributions to noise. J. Am. Soc. Spectrom. 12, 1020-1035 (2001).

46. X, G. et al. Serines 13 and 16 are critical determinants of full-length human mutant huntingtin induced disease pathogenesis in HD mice. Neuron 64, (00:00:00.0).

47. Chiti, F. \& Dobson, C. M. Protein Misfolding, Functional Amyloid, and Human Disease. Annu. Rev. Biochem. 75, 333-366 (2006).

48. Kar, K., Jayaraman, M., Sahoo, B., Kodali, R. \& Wetzel, R. Critical nucleus size for diseaserelated polyglutamine aggregation is repeat length dependent. Nat. Struct. Mol. Biol. 18, 328336 (2011).

49. Scherzinger, E. et al. Self-assembly of polyglutamine-containing huntingtin fragments into amyloid-like fibrils: implications for Huntington's disease pathology. Proc. Natl. Acad. Sci. U.S.A. 96, 4604-4609 (1999).

50. Jayaraman, M. et al. Slow amyloid nucleation via $\alpha$-helix-rich oligomeric intermediates in short polyglutamine-containing huntingtin fragments. J. Mol. Biol. 415, 881-899 (2012).

51. Olshina, M. A. et al. Tracking mutant huntingtin aggregation kinetics in cells reveals three major populations that include an invariant oligomer pool. J. Biol. Chem. 285, 21807-21816 (2010).

52. Bulone, D., Masino, L., Thomas, D. J., San Biagio, P. L. \& Pastore, A. The Interplay between PolyQ and Protein Context Delays Aggregation by Forming a Reservoir of Protofibrils. PLoS One 1, (2006).

53. Hoop, C. L. et al. Huntingtin exon 1 fibrils feature an interdigitated $\beta$-hairpin-based polyglutamine core. Proc. Natl. Acad. Sci. U.S.A. 113, 1546-1551 (2016).

54. Hands, S. L. \& Wyttenbach, A. Neurotoxic protein oligomerisation associated with polyglutamine diseases. Acta Neuropathol. 120, 419-437 (2010).

55. Xi, W., Wang, X., Laue, T. M. \& Denis, C. L. Multiple discrete soluble aggregates influence polyglutamine toxicity in a Huntington's disease model system. Sci. Rep. 6, (2016).

56. Bonfanti, S. et al. Molecular mechanisms of heterogeneous oligomerization of huntingtin proteins. Sci. Rep. 9, 7615 (2019).

57. Sahoo, B. et al. Folding Landscape of Mutant Huntingtin Exon1: Diffusible Multimers, Oligomers and Fibrils, and No Detectable Monomer. PLoS One 11, (2016). 
58. Kar, K. et al. $\beta$-hairpin-mediated nucleation of polyglutamine amyloid formation. J. Mol. Biol. 425, 1183-1197 (2013).

59. Lin, H.-K. et al. Fibril polymorphism affects immobilized non-amyloid flanking domains of huntingtin exon1 rather than its polyglutamine core. Nat. Commun. 8, 1-12 (2017).

60. Shen, K. et al. Control of the structural landscape and neuronal proteotoxicity of mutant Huntingtin by domains flanking the polyQ tract. eLife $\mathbf{5}$,

61. Fodale, V. et al. Polyglutamine- and Temperature-Dependent Conformational Rigidity in Mutant Huntingtin Revealed by Immunoassays and Circular Dichroism Spectroscopy. PLoS ONE 9, e112262 (2014).

62. Gao, X. et al. Cholesterol Modifies Huntingtin Binding to, Disruption of, and Aggregation on Lipid Membranes. Biochemistry 55, 92-102 (2016).

63. Beasley, M. et al. Lipid membranes influence the ability of small molecules to inhibit huntingtin fibrillization. Biochemistry (2019) doi:10.1021/acs.biochem.9b00739.

64. Chaibva, M. et al. Acetylation within the First 17 Residues of Huntingtin Exon 1 Alters Aggregation and Lipid Binding. Biophys. J. 111, 349-362 (2016).

65. Todd, T. W. \& Lim, J. Aggregation Formation in the Polyglutamine Diseases: Protection at a Cost? Mol. Cells 36, 185-194 (2013).

66. Stefani, M. \& Dobson, C. M. Protein aggregation and aggregate toxicity: new insights into protein folding, misfolding diseases and biological evolution. J. Mol. Med. 81, 678-699 (2003).

67. Arrasate, M. \& Finkbeiner, S. Protein aggregates in Huntington's disease. Exp. Neurol. 238, $1-11(2012)$.

68. Arrasate, M., Mitra, S., Schweitzer, E. S., Segal, M. R. \& Finkbeiner, S. Inclusion body formation reduces levels of mutant huntingtin and the risk of neuronal death. Nature 431, $805-810$ (2004).

69. Lajoie, P. \& Snapp, E. L. Formation and toxicity of soluble polyglutamine oligomers in living cells. PLOS ONE 5, e15245 (2010).

70. Nucifora, L. G. et al. Identification of novel potentially toxic oligomers formed in vitro from mammalian-derived expanded huntingtin exon-1 protein. J. Biol. Chem. 287, 16017-16028 (2012).

71. Kim, Y. E. et al. Soluble Oligomers of PolyQ-Expanded Huntingtin Target a Multiplicity of Key Cellular Factors. Mol. Cell 63, 951-964 (2016).

72. Pieri, L., Madiona, K., Bousset, L. \& Melki, R. Fibrillar $\alpha$-Synuclein and Huntingtin Exon 1 Assemblies Are Toxic to the Cells. Biophys. J. 102, 2894-2905 (2012). 
73. Drombosky, K. W. et al. Mutational analysis implicates the amyloid fibril as the toxic entity in Huntington's disease. Neurobiol. Dis. 120, 126-138 (2018).

74. Saudou, F., Finkbeiner, S., Devys, D. \& Greenberg, M. E. Huntingtin acts in the nucleus to induce apoptosis but death does not correlate with the formation of intranuclear inclusions. Cell 95, 55-66 (1998).

75. Leitman, J., Hartl, F. U. \& Lederkremer, G. Z. Soluble forms of polyQ-expanded huntingtin rather than large aggregates cause endoplasmic reticulum stress. Nat. Commun. 4, 1-10 (2013).

76. Ramdzan, Y. M. et al. Huntingtin Inclusions Trigger Cellular Quiescence, Deactivate Apoptosis, and Lead to Delayed Necrosis. Cell Rep. 19, 919-927 (2017).

77. Jayaraman, M. et al. Kinetically competing huntingtin aggregation pathways control amyloid polymorphism and properties. Biochemistry 51, 2706-2716 (2012).

78. Michalek, M., Salnikov, E. S. \& Bechinger, B. Structure and Topology of the Huntingtin 117 Membrane Anchor by a Combined Solution and Solid-State NMR Approach. Biophys. J. 105, 699-710 (2013).

79. Michalek, M., Salnikov, E. S., Werten, S. \& Bechinger, B. Membrane Interactions of the Amphipathic Amino Terminus of Huntingtin. Biochemistry 52, 847-858 (2013).

80. Mishra, R. et al. Inhibiting the nucleation of amyloid structure in a huntingtin fragment by targeting $\alpha$-helix-rich oligomeric intermediates. J. Mol. Biol. 415, 900-917 (2012).

81. Brass, V. et al. An amino-terminal amphipathic alpha-helix mediates membrane association of the hepatitis C virus nonstructural protein 5A. J. Biol. Chem. 277, 8130-8139 (2002).

82. Georgieva, E. R., Xiao, S., Borbat, P. P., Freed, J. H. \& Eliezer, D. Tau binds to lipid membrane surfaces via short amphipathic helices located in its microtubule-binding repeats. Biophys. J. 107, 1441-1452 (2014).

83. Cui, H., Lyman, E. \& Voth, G. A. Mechanism of Membrane Curvature Sensing by Amphipathic Helix Containing Proteins. Biophys. J. 100, 1271-1279 (2011).

84. Chaibva, M., Burke, K. A. \& Legleiter, J. Curvature Enhances Binding and Aggregation of Huntingtin at Lipid Membranes. Biochemistry 53, 2355-2365 (2014).

85. Cong, X. et al. Mass spectrometric identification of novel lysine acetylation sites in huntingtin. Mol. Cell Proteomics 10, M111.009829 (2011).

86. Steffan, J. S. et al. SUMO modification of Huntingtin and Huntington's disease pathology. Science 304, 100-104 (2004).

87. O'Rourke, J. G. et al. SUMO-2 and PIAS1 Modulate Insoluble Mutant Huntingtin Protein Accumulation. Cell Rep. 4, 362-375 (2013). 
88. DeGuire, S. M. et al. N-terminal Huntingtin (Htt) phosphorylation is a molecular switch regulating Htt aggregation, helical conformation, internalization, and nuclear targeting. $J$. Biol. Chem. 293, 18540-18558 (2018).

89. Thompson, L. M. et al. IKK phosphorylates Huntingtin and targets it for degradation by the proteasome and lysosome. J. Cell Biol. 187, 1083-1099 (2009).

90. Ceccon, A. et al. Interaction of Huntingtin Exon-1 Peptides with Lipid-Based Micellar Nanoparticles Probed by Solution NMR and Q-Band Pulsed EPR. J. Am. Chem. Soc. 140, 6199-6202 (2018).

91. Sedighi, F., Adegbuyiro, A. \& Legleiter, J. SUMOylation Prevents Huntingtin Fibrillization and Localization onto Lipid Membranes. ACS Chem. Neurosci. 11, 328-343 (2020).

92. Borrell-Pagès, M., Zala, D., Humbert, S. \& Saudou, F. Huntington's disease: from huntingtin function and dysfunction to therapeutic strategies. Cell. Mol. Life Sci. 63, 2642-2660 (2006).

93. Dorval, V. \& Fraser, P. E. SUMO on the road to neurodegeneration. Biochim. Biophys. Acta 1773, 694-706 (2007).

94. Aiken, C. T. et al. Phosphorylation of threonine 3: implications for Huntingtin aggregation and neurotoxicity. J. Biol. Chem. 284, 29427-29436 (2009).

95. Pennuto, M., Palazzolo, I. \& Poletti, A. Post-translational modifications of expanded polyglutamine proteins: impact on neurotoxicity. Hum. Mol. Genet. 18, R40-47 (2009).

96. Arndt, J. R., Brown, R. J., Burke, K. A., Legleiter, J. \& Valentine, S. J. Lysine residues in the $\mathrm{N}$-terminal huntingtin amphipathic $\alpha$-helix play a key role in peptide aggregation. J. Mass Spectrom. 50, 117-126 (2015).

97. Atwal, R. S. et al. Kinase inhibitors modulate huntingtin cell localization and toxicity. Nat. Chem. Biol. 7, 453-460 (2011).

98. Chiki, A. et al. Mutant Exon1 Huntingtin Aggregation is Regulated by T3 PhosphorylationInduced Structural Changes and Crosstalk between T3 Phosphorylation and Acetylation at K6. Angew. Chem. Int. Ed. Engl. 56, 5202-5207 (2017).

99. Cariulo, C. et al. Phosphorylation of huntingtin at residue T3 is decreased in Huntington's disease and modulates mutant huntingtin protein conformation. Proc. Natl. Acad. Sci. U.S.A. 114, E10809-E10818 (2017).

100. Trushina, E. et al. Mutant Huntingtin Impairs Axonal Trafficking in Mammalian Neurons In Vivo and In Vitro. Mol. Cell Biol. 24, 8195-8209 (2004).

101. Dragatsis, I., Levine, M. S. \& Zeitlin, S. Inactivation of Hdh in the brain and testis results in progressive neurodegeneration and sterility in mice. Nat. Genet. 26, 300-306 (2000). 
102. Atwal, R. S. et al. Huntingtin has a membrane association signal that can modulate huntingtin aggregation, nuclear entry and toxicity. Hum. Mol. Genet. 16, 2600-2615 (2007).

103. Hughes, A. \& Jones, L. Huntingtin localisation studies - a technical review. PLoS Curr. 3 , (2011).

104. Kegel, K. B. et al. Huntingtin Associates with Acidic Phospholipids at the Plasma Membrane. J. Biol. Chem. 280, 36464-36473 (2005).

105. DiFiglia, M. et al. Huntingtin is a cytoplasmic protein associated with vesicles in human and rat brain neurons. Neuron 14, 1075-1081 (1995).

106. Gutekunst, C. A. et al. Identification and localization of huntingtin in brain and human lymphoblastoid cell lines with anti-fusion protein antibodies. Proc. Natl. Acad. Sci. U.S.A. 92, 8710-8714 (1995).

107. Sharp, A. H. et al. Widespread expression of Huntington's disease gene (IT15) protein product. Neuron 14, 1065-1074 (1995).

108. Nasir, J. et al. Targeted disruption of the Huntington's disease gene results in embryonic lethality and behavioral and morphological changes in heterozygotes. Cell 81, 811-823 (1995).

109. Zeitlin, S., Liu, J. P., Chapman, D. L., Papaioannou, V. E. \& Efstratiadis, A. Increased apoptosis and early embryonic lethality in mice nullizygous for the Huntington's disease gene homologue. Nat. Genet. 11, 155-163 (1995).

110. O'Kusky, J. R., Nasir, J., Cicchetti, F., Parent, A. \& Hayden, M. R. Neuronal degeneration in the basal ganglia and loss of pallido-subthalamic synapses in mice with targeted disruption of the Huntington's disease gene. Brain Res. 818, 468-479 (1999).

111. Wang, H., Lim, P. J., Karbowski, M. \& Monteiro, M. J. Effects of overexpression of Huntingtin proteins on mitochondrial integrity. Hum. Mol. Genet. 18, 737-752 (2009).

112. Mihm, M. J. et al. Cardiac dysfunction in the R6/2 mouse model of Huntington's disease. Neurobiol. Dis. 25, 297-308 (2007).

113. Velier, J. et al. Wild-Type and Mutant Huntingtins Function in Vesicle Trafficking in the Secretory and Endocytic Pathways. Exp. Neurol. 152, 34-40 (1998).

114. Kb, K. et al. Polyglutamine expansion in huntingtin increases its insertion into lipid bilayers. Biochem. Biophys. Res. Commun. 387, 472-475 (2009).

115. Kegel, K. B. et al. Polyglutamine expansion in huntingtin alters its interaction with phospholipids. J. Neurochem. 110, 1585-1597 (2009). 
116. Burke, K. A., Hensal, K. M., Umbaugh, C. S., Chaibva, M. \& Legleiter, J. Huntingtin disrupts lipid bilayers in a polyQ-length dependent manner. Biochim. Biophys. Acta, Biomem. 1828, 1953-1961 (2013).

117. Slepko, N. et al. Normal-repeat-length polyglutamine peptides accelerate aggregation nucleation and cytotoxicity of expanded polyglutamine proteins. Proc. Natl. Acad. Sci. U.S.A. 103, 14367-14372 (2006).

118. Suopanki, J. et al. Interaction of huntingtin fragments with brain membranes - clues to early dysfunction in Huntington's disease. J. Neurochem. 96, 870-884 (2006).

119. Guedes-Dias, P. \& Holzbaur, E. L. F. Huntingtin Fibrils Poke Membranes. Cell 171, 32-33 (2017).

120. Valencia, A. et al. Mutant huntingtin and glycogen synthase kinase 3-beta accumulate in neuronal lipid rafts of a presymptomatic knock-in mouse model of Huntington's disease. $J$. Neurosci. Res. 88, 179-190 (2010).

121. Kegel, K. B. et al. Huntingtin expression stimulates endosomal-lysosomal activity, endosome tubulation, and autophagy. J. Neurosci. 20, 7268-7278 (2000).

122. Qin, Z.-H. et al. Huntingtin bodies sequester vesicle-associated proteins by a polyprolinedependent interaction. J. Neurosci. 24, 269-281 (2004).

123. Cooke, I. R. \& Deserno, M. Coupling between Lipid Shape and Membrane Curvature. Biophys. J. 91, 487-495 (2006).

124. Alberts, B. et al. The Lipid Bilayer. Molecular Biology of the Cell. 4th edition (2002).

125. Bennett, W. F. D. \& Tieleman, D. P. The Importance of Membrane Defects-Lessons from Simulations. Acc. Chem. Res. 47, 2244-2251 (2014).

126. Arndt, J. R., Chaibva, M. \& Legleiter, J. The emerging role of the first 17 amino acids of huntingtin in Huntington's disease. Biomol. Concepts 6, 33-46 (2015).

127. Haque, Md. E., McIntosh, T. J. \& Lentz, B. R. Influence of Lipid Composition on Physical Properties and PEG-Mediated Fusion of Curved and Uncurved Model Membrane Vesicles: “Nature's Own” Fusogenic Lipid Bilayer. Biochemistry 40, 4340-4348 (2001).

128. Chaibva, M. et al. Sphingomyelin and GM1 Influence Huntingtin Binding to, Disruption of, and Aggregation on Lipid Membranes. ACS Omega 3, 273-285 (2018).

129. Bigay, J. \& Antonny, B. Curvature, Lipid Packing, and Electrostatics of Membrane Organelles: Defining Cellular Territories in Determining Specificity. Dev. Cell 23, 886-895 (2012). 
130. Burke, K. A., Yates, E. A. \& Legleiter, J. Biophysical Insights into How Surfaces, Including Lipid Membranes, Modulate Protein Aggregation Related to Neurodegeneration. Front. Neurol. 4, (2013).

131. Pandey, N. K. et al. The 17-residue-long N terminus in huntingtin controls stepwise aggregation in solution and on membranes via different mechanisms. J. Biol. Chem. 293, 2597-2605 (2018).

132. Marquette, A. \& Bechinger, B. Membrane interactions accelerate the self-aggregation of huntingtin exon 1 fragments in a polyglutamine length-dependent manner. bioRxiv 2020.06.24.169060 (2020) doi:10.1101/2020.06.24.169060.

133. Karanji, A. K. et al. Investigating the interactions of the first 17 amino acid residues of Huntingtin with lipid vesicles using mass spectrometry and molecular dynamics. J. Mass Spectrom. 55, e4470 (2020).

134. Fenn, J. B. Electrospray Wings for Molecular Elephants (Nobel Lecture). Angew. Chem. Int. 42, 3871-3894 (2003).

135. Kebarle, P. \& Verkerk, U. H. Electrospray: From ions in solution to ions in the gas phase, what we know now. Mass Spectrom. Rev. 28, 898-917 (2009).

136. Cole, R. Electrospray and MALDI Mass Spectrometry: Fundamentals, Instrumentation, Practicalities, and Biological Applications, 2nd Edition | Wiley. (2010).

137. Dole, M. et al. Molecular Beams of Macroions. J. Chem. Phys. 49, 2240-2249 (1968).

138. Leney, A. C. \& Heck, A. J. R. Native Mass Spectrometry: What is in the Name? J. Am. Soc. Mass. Spectrom. 28, 5-13 (2017).

139. Electrospray Ionization Mass Spectrometry. Chemistry LibreTexts https://chem.libretexts.org/Bookshelves/Analytical_Chemistry/Supplemental_Modules_(Anal ytical_Chemistry)/Instrumental_Analysis/Mass_Spectrometry/Mass_Spectrometers_(Instrume ntation)/Electrospray_Ionization_Mass_Spectrometry (2013).

140. Banerjee, S. \& Mazumdar, S. Electrospray Ionization Mass Spectrometry: A Technique to Access the Information beyond the Molecular Weight of the Analyte. Int. J. Anal. Chem. vol. 2012 e282574 https://www.hindawi.com/journals/ijac/2012/282574/ (2012).

141. Binnig, G., Quate, C. F. \& Gerber, Ch. Atomic Force Microscope. Phys. Rev. Lett. 56, 930933 (1986).

142. AFM Worksop, USA. AFM and SEM Benefits - Scanning Electron and Atomic Force Microscope. https://www.afmworkshop.com/newsletter/258-sem-afm-are-complementary.

143. Kollmer, M. et al. Electron tomography reveals the fibril structure and lipid interactions in amyloid deposits. Proc. Natl. Acad. Sci. U.S.A. 113, 5604-5609 (2016). 
144. Newcomb, C. J., Moyer, T. J., Lee, S. S. \& Stupp, S. I. Advances in cryogenic transmission electron microscopy for the characterization of dynamic self-assembling nanostructures. Curr. Opin. Colloid Interface Sci. 17, 350-359 (2012).

145. Zhang, Y. et al. Quantitating morphological changes in biological samples during scanning electron microscopy sample preparation with correlative super-resolution microscopy. PLoS One 12, (2017).

146. The Differences Between Atomic Force Microscopy and Scanning Electron Microscopy. AZoM.com https://www.azom.com/article.aspx?ArticleID=11879 (2015).

147. Legleiter, J. \& Kowalewski, T. Atomic force microscopy of beta-amyloid: static and dynamic studies of nanostructure and its formation. Methods Mol. Biol. 242, 349-364 (2004).

148. Blackley, H. K. L. et al. Morphological Development of $\beta(1-40)$ Amyloid Fibrils. Exp. Neurol. 158, 437-443 (1999).

149. Pilkington, A. W. et al. Hydrogen Peroxide Modifies A $\beta-M e m b r a n e$ Interactions with Implications for A 440 Aggregation. Biochemistry 58, 2893-2905 (2019).

150. Burke, K. A., Yates, E. A. \& Legleiter, J. Amyloid-Forming Proteins Alter the Local Mechanical Properties of Lipid Membranes. Biochemistry 52, 808-817 (2013).

151. Legleiter, J., Fryer, J. D., Holtzman, D. M. \& Kowalewski, T. The Modulating Effect of Mechanical Changes in Lipid Bilayers Caused by ApoE-Containing Lipoproteins on A $\beta$ Induced Membrane Disruption. ACS Chem. Neurosci. 2, 588-599 (2011).

152. Legleiter, J., Park, M., Cusick, B. \& Kowalewski, T. Scanning probe acceleration microscopy (SPAM) in fluids: Mapping mechanical properties of surfaces at the nanoscale. Proc. Natl. Acad. Sci. U.S.A. 103, 4813-4818 (2006).

153. Putman, C. A. J., Van der Werf, K. O., De Grooth, B. G., Van Hulst, N. F. \& Greve, J. Tapping mode atomic force microscopy in liquid. Appl. Phys. Lett. 64, 2454-2456 (1994).

154. Li, H.-Q. AFM Scanning Modes. The Common AFM Modes http://www.chembio.uoguelph.ca/educmat/chm729/afm/details.htm.

155. Albrecht, T. R., Grütter, P., Horne, D. \& Rugar, D. Frequency modulation detection using high-Q cantilevers for enhanced force microscope sensitivity. J. Appl. Phys. 69, 668-673 (1991).

156. Bruker Corporation. AFM Modes. Bruker nanoscale https://www.nanophys.kth.se/nanolab/afm/icon/brukerhelp/Content/SPM\%20Training\%20Guide/Atomic\%20Force\%20Microscopy\%20(AFM)/AF M\%20Modes.htm.

157. Created with BioRender.com. 


\section{Normalizing Polydiacetylene Colorimetric Assays of Vesicle Binding Across Lipid Systems ${ }^{1}$}

Numerous life processes occur at cellular and subcellular membranes, predominately comprised of lipids. Determining the binding affinity of proteins and other molecules to lipid membranes provides insight into cell signaling, transport, energy metabolism, disease progression, and drug discovery. Mixed polydiacetylene (PDA) lipid vesicles mimic cell membranes and exhibit a colorimetric response induced by mechanical stress, which can be associated with the binding of proteins or molecules. Due to its simple spectroscopic readout, PDA assays are amenable to high-throughput screens. However, PDA assays exhibit batch to batch variability and the sensitivity of the assay is influenced by the physicochemical properties of lipid vesicles. Here, a method of normalizing PDA lipid binding assays to reduce variability and enable direct comparison of different lipid systems is described. Normalization of PDA sensitivity reduced variability between batches of PDA/lipid systems of DOPC, POPC, POPE, and POPG by upwards of $78 \%$, providing a cost-effective, simple, and reproducible method of determining relative membrane binding affinity for various molecules with the potential for highthroughput screening.

\subsection{Introduction}

Lipids are the dominant component of cellular membranes and have numerous critical biological functions as signaling molecules, ${ }^{1}$ transporters, ${ }^{2}$ protein recruiters, ${ }^{3}$ energy sources, ${ }^{4}$ and initiators of cell-to-cell interaction. ${ }^{5}$ Eukaryotic organisms contain a dozen major lipid

\footnotetext{
${ }^{1}$ This article/chapter was published in Analytical Biochemistry, 609, Beasley, M., Stonebraker A., Legleiter, J., Normalizing polydiacetylene colorimetric assays of vesicle binding across lipid systems, Copyright Elsevier (2020) 
classes that are comprised of hundreds of individual species ${ }^{6,7}$ and the lipid composition of membranes directly impact their function. Varying lipid composition alters physicochemical properties of membranes, ${ }^{8}$ changing membrane structure, fluidity, charge density, and interactions with various molecules. ${ }^{5}$ These variations significantly impact membrane function. ${ }^{9,10}$ Many proteins directly interact with membranes as part of performing various cellular processes, such as vesicular trafficking, and undergo structural transitions associated with regulating biological activity of peptides such as peptide hormones, antimicrobial peptide functions, and amyloid formation. ${ }^{11}$ In addition, membrane binding is an important step for many drugs to reach their biological target, so the affinity of pharmaceuticals to membranes impacts drug delivery and uptake. ${ }^{2}$ As membrane affinity of proteins and small molecules varies with lipid composition, there is a need to efficiently measure and directly compare interactions between molecules and different lipid systems.

Several techniques measure the binding affinity of molecules to different membranes, but many are costly, inaccurate, or lack the ability for high throughput. The n-octanol/buffer partition system is commonly used to determine the membrane affinity of drugs; however, it does not accurately model electrostatic interactions associated with the negative charge of membranes. ${ }^{12}$ While implementation of a liposome/buffer system via equilibrium dialysis can overcome this issue, this method is not amenable for high-throughput experimentation as equilibrium dialysis chambers contain few reaction wells and experiments require long periods of incubation. ${ }^{13}$ Chromatographic techniques, such as immobilized artificial membrane chromatography, are capable of measuring lipid binding affinities, but these methods often suffer from low cross-column reproducibility. ${ }^{14}$ Surface plasmon resonance (SPR) techniques are highly accurate and allow for real-time monitoring of the binding of peptides to an immobilized 
lipid bilayer with high throughput experiments; however, the method is expensive. ${ }^{15}$ An SPR instrument capable of probing 36 reactions at once costs over an order of magnitude more than a multimode microplate reader capable of analyzing 384 samples at once, and a single biosensor chip for SPR experiments can cost around \$250 (approximately $\$ 7$ per data point). ${ }^{16,17}$

Polydiacetylene/lipid systems have the potential to qualitatively measure membrane affinity in a simple, cost effective, and high-throughput manner. Polydiacetylene (PDA) is a polymer that displays a colorimetric shift (from blue to red) when the conjugated backbone experiences a stress or structural perturbation. This colorimetric response can be quantitatively measured via absorption or fluorescence spectroscopy. Stress applied to the system by stimuli such as temperature, ${ }^{18} \mathrm{pH},{ }^{19}$ proteins or peptides, ${ }^{20,21}$ small molecules, ${ }^{22}$ and metal ions ${ }^{23}$ can invoke the colorimetric response. With regards to the interaction of molecules to lipid membranes, the colorimetric response is quantitatively correlated to membrane affinity in a reproducible manner. ${ }^{24}$ As diacetylene monomers are inexpensive, PDA/lipid vesicles very closely mimic bilayer environments within cellular membranes, ${ }^{25}$ and the colorimetric response is easily measured in a multi-well plate format, PDA assays are an ideal option for cost effective, accurate, high-throughput measurement of the interactions of molecules and peptides with lipid membranes. $^{26-28}$

While PDA assays are often used to compare the binding of different molecules to a lipid system, ${ }^{29,30}$ it has not been used to compare how molecules bind multiple different lipid systems. Lipid composition influences the colorimetric response for a set amount of stress as a factor of fluidity, making direct comparison of different PDA/lipid systems challenging. ${ }^{25}$ This paper presents a method of data analysis and normalization of the sensitivity of PDA assays for four lipid systems of different fluidity and charge: 1,2-dioleoyl-sn-glycero-3-phosphocholine 
(DOPC), 1-palmitoyl-2-oleoyl-glycero-3-phosphocholine (POPC), 1-palmitoyl-2-oleoyl-snglycero-3-phosphoethanolamine (POPE), and 1-palmitoyl-2-oleoyl-sn-glycero-3-phospho-(1'rac-glycerol) (POPG). The presented method enables PDA/lipid binding assays to be used as a reliable technique to directly compare the membrane interaction and binding of molecules, drugs, and peptides to various lipid systems.

\subsection{Materials and Methods}

\subsubsection{Materials}

1,2-dioleoyl-sn-glycero-3-phosphocholine (DOPC), 1-palmitoyl-2-oleoyl-glycero-3phosphocholine (POPC), 1-palmitoyl-2-oleoyl-sn-glycero-3-phosphoethanolamine (POPE), and 1-palmitoyl-2-oleoyl-sn-glycero-3-phospho-(1'-rac-glycerol) (POPG) were purchased from Avanti Polar Lipids. Diacetylene monomers of 10,12-tricosadiynoic acid for the PDA assays were purchased from GFS Chemicals (Columbus, OH). Small molecules (-)-epigallocatechin-3gallate (EGCG) and riluzole were purchased from Acros Organics. Synthetic $\beta$-amyloid 1-40 was purchased from AnaSpec Inc. (San Jose, CA) and Alfa Aesar (Tewksbury, MA). All data was collected on a SpectraMax M2 microplate reader.

\subsubsection{Preparation of lipid/PDA vesicles}

Lipid and 10,12-tricosadiynoic acid were mixed at a 2:3 molar ratio in a chloroform/ethanol (5:1) solution and dried with a gentle stream of nitrogen until a film was formed. Once fully dried, the film was reconstituted in $70{ }^{\circ} \mathrm{C}$ buffer $(150 \mathrm{mM} \mathrm{NaCl}$, Tris- $\mathrm{HCl}$, $\mathrm{pH}$ 7.4) and probe-sonicated at $125 \mathrm{~W}$ for $10 \mathrm{~min}$. The opaque solution was then protected from light and stored overnight at $4{ }^{\circ} \mathrm{C}$ to allow the lipids to self-associate into vesicles. The following 
day, the solution was gradually brought to room temperature and irradiated with $254 \mathrm{~nm}$ light for 10 min with constant stirring. The resulting royal blue solution was stored at $4{ }^{\circ} \mathrm{C}$ before use.

\subsubsection{Preparation of EGCG}

Powdered EGCG $(4.5 \mathrm{mg})$ was dissolved in $1 \mathrm{~mL}$ DMSO to create a $10 \mathrm{mM}$ stock solution. Stock solutions of 7.5, 5, 2.5, and $1 \mathrm{mM} \mathrm{EGCG} \mathrm{were} \mathrm{formed} \mathrm{from} \mathrm{the} \mathrm{stock} \mathrm{solution}$ through serial dilution. For each experiment, $1 \mu \mathrm{L}$ was added to each $100 \mu \mathrm{L}$ well to result in final EGCG concentrations of 100, 75, 50, 25, and $10 \mu \mathrm{M}$ EGCG.

\subsubsection{Preparation of $\beta$-amyloid peptide}

Synthetic $\beta$-amyloid 1-40 $\left(\mathrm{A} \beta_{40}\right)$ peptide was equilibrated to room temperature and then dissolved in HFIP for aliquoting. Aliquots were left in a fume hood for $1 \mathrm{~h}$ to allow the evaporation of the HFIP, and then the samples were dried in vacuum overnight to remove residual HFIP. Dried films were stored at $-20{ }^{\circ} \mathrm{C}$ until needed. On the day of the experiment, the $\mathrm{A} \beta_{40}$ films were equilibrated to room temperature and then dissolved in DMSO. Stocks were prepared to $2 \mathrm{mM}$, and $1 \mu \mathrm{L}$ of the stock solution was added to each $100 \mu \mathrm{L}$ well for a final $\mathrm{A} \beta$ concentration of $20 \mu \mathrm{M}$.

\subsubsection{Determination of colorimetric response}

PDA/lipid assays were performed in a 96-well plate format $(100 \mu \mathrm{L}$ total volume per well) with a PDA/lipid vesicle concentration of $400 \mu \mathrm{M}$ using a SpectraMax M2 microplate reader at $30^{\circ} \mathrm{C}$. The absorbance at blue $(640 \mathrm{~nm})$ and red $(550 \mathrm{~nm})$ wavelengths were recorded and used to calculate the percent colorimetric response $(\% \mathrm{CR})$ : 


$$
\% \text { colorimetric response }=\left(P B_{0}-P B_{1} / P B_{0}\right) \times 100
$$

where $P B={ }^{A 40} / A_{640}+A_{550}$ for the negative (blank) control $\left(\mathrm{PB}_{0}\right)$ or the sample ( $\left.\mathrm{PB}_{1}\right)$. Each condition was performed in triplicate, and reported values are averages of these replicates. For each experiment, a negative control was included that consisted of 50:50 PDA/lipid vesicles and neat buffer. Each experiment was completed a minimum of three times with unique batches of lipid/PDA vesicles.

\subsubsection{Assay normalization and analysis}

$\mathrm{Lipid} / \mathrm{PDA}$ vesicles were exposed to $100 \mathrm{mM} \mathrm{NaOH}$ for $3 \mathrm{~h}$ to obtain the maximum \% CR for each batch of lipid/PDA vesicles. The maximum potential \% CR for each system was defined as the maximum value of the steady state of the kinetic curve. The steady state was determined to begin at the measurement where the signal did not change beyond $\pm 5 \%$ of the colorimetric response for at least one hour. The $5 \%$ change was selected as the determining factor because it demonstrates little to no further increase in colorimetric response, while still allowing for the potential of noise in the assay. For normalization, the $\mathrm{CR}$ for each assay was normalized to the maximum $\% \mathrm{CR}$ to determine the relative $\% \mathrm{CR}$ for each system:

$$
\text { relative } \% \text { colorimetric response }=\left({ }^{\% C R_{\text {sample }}} / \% C R_{\max }\right) \times 100
$$

where $\% \mathrm{CR}_{\max }$ is the steady state maximum $\mathrm{CR}$ associated with the $\mathrm{NaOH}$ standard. The between-batch relative standard deviation (RSD) was calculated to determine the reproducibility for the same lipid system, and between-system RSD was calculated to determine the variability between lipid systems. 


\subsection{Results and Discussion}

\subsubsection{Colorimetric response is linearly correlated to applied stress}

To establish a relationship between mechanical stress and \% CR, a series of PDA assays were performed with DOPC, POPC, POPE, and POPG vesicles exposed to various doses of $\mathrm{NaOH}$ (up to $200 \mathrm{mM}$; Fig. 2.1a). $\mathrm{NaOH}$ stresses the systems by increasing repulsion among lipid head groups within the vesicles, invoking a colorimetric response. ${ }^{19,31}$ For each lipid system, \% CR increased in a dose dependent manner with $\mathrm{NaOH}$ until the $45 \mathrm{mM}$ exposure, saturating the response and resulting in a working range up to $35 \mathrm{mM} \mathrm{NaOH}$. The magnitude of the \% CR varied from batch-to-batch and across lipid systems (Fig. 2.2). With $45 \mu \mathrm{M}$ doses of $\mathrm{NaOH}$, a steady state $\mathrm{CR}$ was reached within an hour, and increasing $\mathrm{NaOH}$ concentration beyond $45 \mu \mathrm{M}$ reduced the time to reach a steady state response. The background noise in the $\%$ CR ranged from 0.3 to $1.2 \%$ across all lipid systems and the maximum signal to noise ratio (based on saturated \% CR) varied from batch-to-batch and across lipid systems (Table 2.1). Within the working range, the maximum $\% \mathrm{CR}$ responded linearly with increasing $\mathrm{NaOH}$ dose across all lipid systems; however, batch-to-batch variability within single lipid systems was observed (Fig. 2.1b). For example, batch 1 of PDA/DOPC vesicles were significantly less sensitive to $\mathrm{NaOH}$ compared to batches 2 and 3. In addition, the doses of $\mathrm{NaOH}$ used here result in a relatively large mechanical stress on the vesicles compared to many systems of interests, i.e. proteins and small molecules. This suggests that, beyond application as a simple positive control, $\mathrm{NaOH}$ exposure can be used as a method to calibrate the response of PDA/lipid vesicles. Such a method would facilitate comparison between unique preparations of PDA/lipid vesicles of the same lipid and potentially allow for relative comparisons between PDA/lipid vesicles comprised of different lipids. 
a
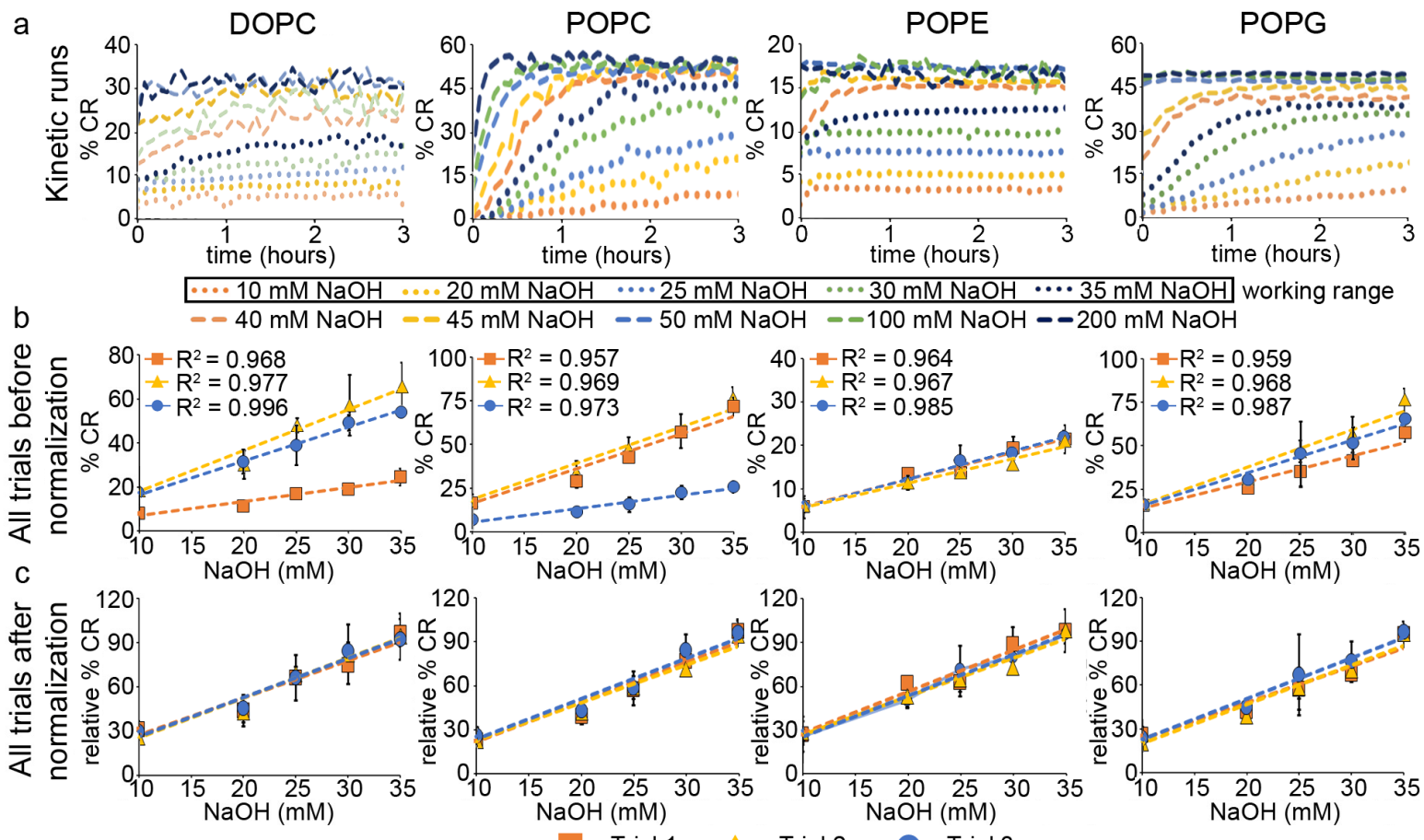

Figure 2.1 PDA normalization reduces assay variability. (a) Kinetic curves of the \% CR of PDA/lipid systems of DOPC, POPC, POPE, and POPG exposed to $\mathrm{NaOH}$. Comparison of the maximum \% CR for different PDA/lipid systems as a function of $\mathrm{NaOH}$ dose (b) before and (c) after normalization. Data points are the average of independent triplicates of each condition, and error bars represent standard error.
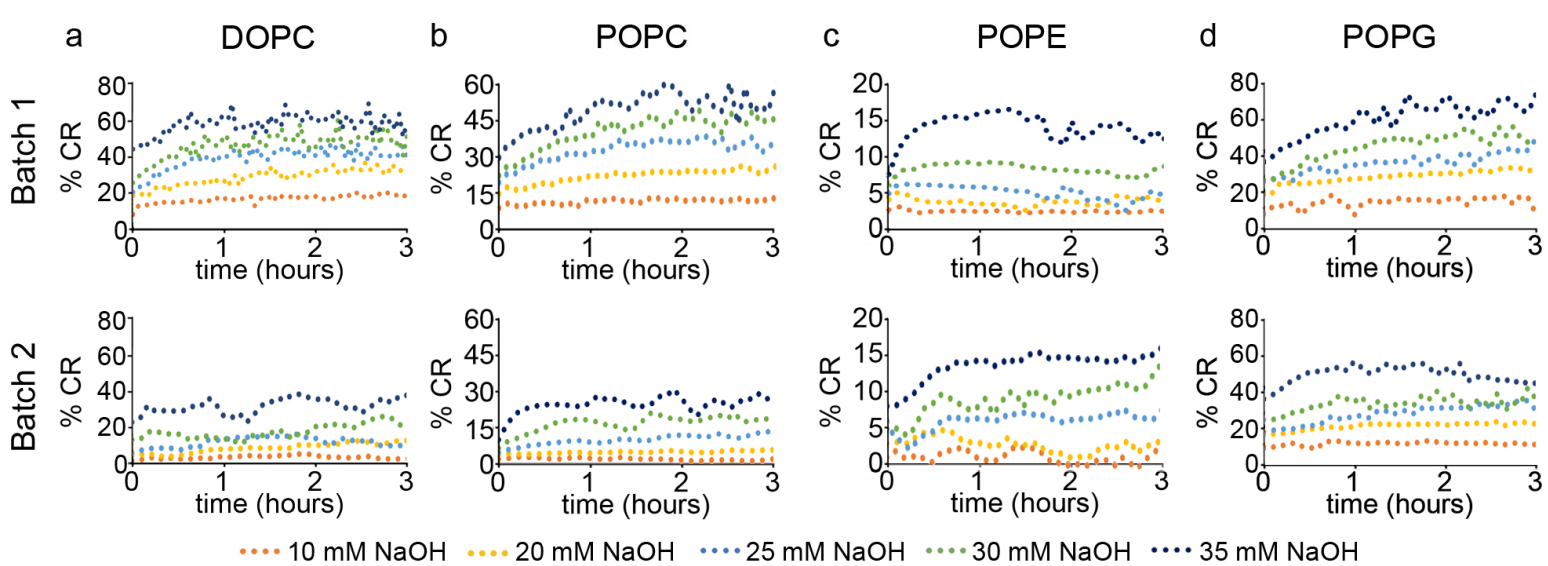

Figure 2.2 The response of PDA/lipid vesicles to $\mathrm{NaOH}$ varies batch to batch. \% $\mathrm{CR}$ kinetic curves for different batches of PDA/lipid vesicles consisting of (a) DOPC, (b) POPC, (c) POPE, and (d) POPG exposed to various doses of $\mathrm{NaOH}$. 
Table 2.1 Maximum S/N for three batches of each PDA/lipid system.

\begin{tabular}{l|lcccc}
\hline \hline \multicolumn{1}{c}{ DOPC } & Background noise & 1.1 & 0.5 & 1.0 & Mean \\
& Maximum S/N & $\mathbf{6 2 . 0}$ & $\mathbf{4 8 . 9}$ & $\mathbf{5 9 . 2}$ & $\mathbf{5 6 . 7} \pm \mathbf{7 . 0}$ \\
POPC & Background noise & 1.2 & 1.8 & 0.6 & \\
& Maximum S/N & $\mathbf{6 0 . 2}$ & $\mathbf{4 4 . 8}$ & $\mathbf{4 6 . 6}$ & $\mathbf{5 0 . 5} \pm \mathbf{8 . 4}$ \\
POPE & Background noise & 0.6 & 0.4 & 0.3 & \\
& Maximum S/N & $\mathbf{3 5 . 3}$ & $\mathbf{5 2 . 0}$ & $\mathbf{7 6 . 6}$ & $\mathbf{5 4 . 6} \pm \mathbf{2 0 . 8}$ \\
POPG & Background noise & 0.6 & 0.8 & 0.6 & \\
& Maximum S/N & $\mathbf{1 0 1 . 2}$ & $\mathbf{1 0 7 . 5}$ & $\mathbf{1 1 6 . 0}$ & $\mathbf{1 0 8 . 3} \pm \mathbf{7 . 4}$ \\
\hline
\end{tabular}

\subsubsection{Calibration and normalization of colorimetric response enhances cross-batch and lipid-to-lipid reproducibility}

To facilitate the direct comparison of different PDA/lipid preparations, calibration curves from individual batches of PDA/lipid vesicles were normalized, using Eq. 2, to the maximum \% $\mathrm{CR}$ associated with a saturating dose of $\mathrm{NaOH}(100 \mathrm{mM}$; Fig. 2.1c). This normalization significantly reduced batch-to-batch variability (Table 2.2). The between-batch relative standard deviation (RSD) for DOPC/PDA vesicle response ranged from $39.5-48.5 \%$ for each data point prior to normalization, and this was reduced to $2.3-9.3 \%$ (an average reduction in RSD of 89.4\%). The POPC/PDA systems demonstrated a between-batch RSD of $43.8-71.8 \%$ before normalization. Upon normalization, the RSD dropped to $1.2-8.5 \%$ (an average reduction in RSD of 91.1\%). Even for systems that did not display large batch-to-batch heterogeneity (POPE and POPG), normalization reduced variability across batches. For the POPE/PDA system, the RSD values ranged from 2.13 to $11.09 \%$ before normalization, and from 1.53 to $10.97 \%$ after normalization. Despite the reproducibility between the three trials before the correction, the RSD still decreased by an average of $19.50 \%$ after the correction. The POPG/PDA systems also demonstrated strong reproducibility before correction, with RSD values of 7.64-15.49\%. After 
correction, the RSD values decreased by an average of $47.84 \%$ to give values between 1.01 and $8.68 \%$. Thus, a standard dose of $\mathrm{NaOH}$ can be used to normalize PDA assays, allowing for direct comparison between batches.

Table 2.2 Batch to batch relative standard deviation (RSD) values for each PDA/lipid system before and after normalization as a function of $\mathrm{NaOH}$ added.

\begin{tabular}{l|lccccc}
\hline \hline \multicolumn{2}{c}{ NaOH treatment (mM): } & $\mathbf{1 0}$ & $\mathbf{2 0}$ & $\mathbf{2 5}$ & $\mathbf{3 0}$ & $\mathbf{3 5}$ \\
\hline RSD of DOPC & Raw data & 39.5 & 46.9 & 46.6 & 48.5 & 44.3 \\
& Normalized & $\mathbf{9 . 3}$ & $\mathbf{2 . 9}$ & $\mathbf{2 . 3}$ & $\mathbf{6 . 3}$ & $\mathbf{2 . 3}$ \\
RSD of POPC & Raw data & 43.8 & 51.0 & 61.9 & 68.6 & 71.8 \\
& Normalized & $\mathbf{8 . 5}$ & $\mathbf{4 . 7}$ & $\mathbf{1 . 2}$ & $\mathbf{8 . 4}$ & $\mathbf{1 . 3}$ \\
RSD of POPE & Raw data & 8.3 & 10.1 & 10.7 & 11.1 & 2.1 \\
& Normalized & $\mathbf{5 . 4}$ & $\mathbf{1 1 . 0}$ & $\mathbf{7 . 3}$ & $\mathbf{9 . 8}$ & $\mathbf{1 . 5}$ \\
RSD of POPG & Raw data & 7.6 & 10.8 & 15.3 & 15.5 & 14.4 \\
& Normalized & $\mathbf{7 . 2}$ & $\mathbf{6 . 9}$ & $\mathbf{8 . 7}$ & $\mathbf{6 . 0}$ & $\mathbf{1 . 0}$ \\
\hline
\end{tabular}

When comparing different lipid systems, variability in PDA/lipid colorimetric response can be due to differences in prep methods or the physicochemical properties of the vesicles. However, this variation can be accounted for, resulting in similar sensitivity for all systems in response to an external stress. The $\% \mathrm{CR}$ changed linearly as a function of $\mathrm{NaOH}$ concentration for all four lipid systems, with $\mathrm{R}^{2}$ values ranging from 0.9565 to 0.9957 . However, the slopes of the linear fits - indicative of the sensitivity of the PDA/lipid system - varied greatly between different lipid systems. When RSD values were calculated for all the trials of the four lipid systems before normalization, the values ranged from 42.49 to $46.24 \%$ (Table 2.3). Thus, each system demonstrated a linear relationship between colorimetric response and stress on their own, but the results were not directly comparable or consistent between the different lipid systems. After normalization of each lipid system, the between-system RSD decreased to $1.75-14.45 \%$, resulting in a percent reduction of 65.99 to $96.18 \%$ (Table 2.2). Despite varying fluidity, bilayer 
thickness, area per lipid, and charge density between the DOPC, POPC, POPE, and POPG vesicles, all four lipid systems demonstrated similar sensitivity after normalization to a determined stimulus, suggesting that the normalization procedure can facilitate comparisons across lipid systems.

Table 2.3 Between-system relative standard deviation (RSD) values for all PDA/ lipid systems before and after normalization as a function of $\mathrm{NaOH}$ added.

\begin{tabular}{cccccc}
\hline $\begin{array}{c}\text { NaOH treatment } \\
(\mathbf{m M}):\end{array}$ & $\mathbf{1 0}$ & $\mathbf{2 0}$ & $\mathbf{2 5}$ & $\mathbf{3 0}$ & $\mathbf{3 5}$ \\
\hline Raw data & 41.8 & 42.5 & 46.2 & 44.5 & 45.7 \\
Normalized & 13.6 & 14.5 & 8.2 & 8.7 & 1.8 \\
\% improvement & $\mathbf{6 7 . 4}$ & $\mathbf{6 6 . 0}$ & $\mathbf{8 2 . 4}$ & $\mathbf{8 0 . 4}$ & $\mathbf{9 6 . 2}$ \\
\hline
\end{tabular}

\subsubsection{Normalized PDA assays can determine relative binding affinities of small molecules and peptides to lipid membranes}

Despite varying physicochemical properties across DOPC, POPC, POPE, and POPG vesicles, all four lipid systems demonstrated similar sensitivity after normalization (Fig. 2.1C, Table 2.3), suggesting that $\mathrm{NaOH}$ normalization can facilitate comparisons across lipid systems. As a result, the normalization procedure was next applied to measuring relative membrane affinities of small molecules and peptides to vesicles comprised of different lipids (Fig. 2.3). A $100 \mathrm{mM}$ dose of $\mathrm{NaOH}$ was utilized as a standard in separate wells which were monitored simultaneously with kinetic experiments with small molecules or peptides to obtain the maximum \% CR of each PDA/lipid vesicle system. All subsequent measurements using a specific PDA system and batch were made relative to the maximum $\% \mathrm{CR}$ determined by the standard. 

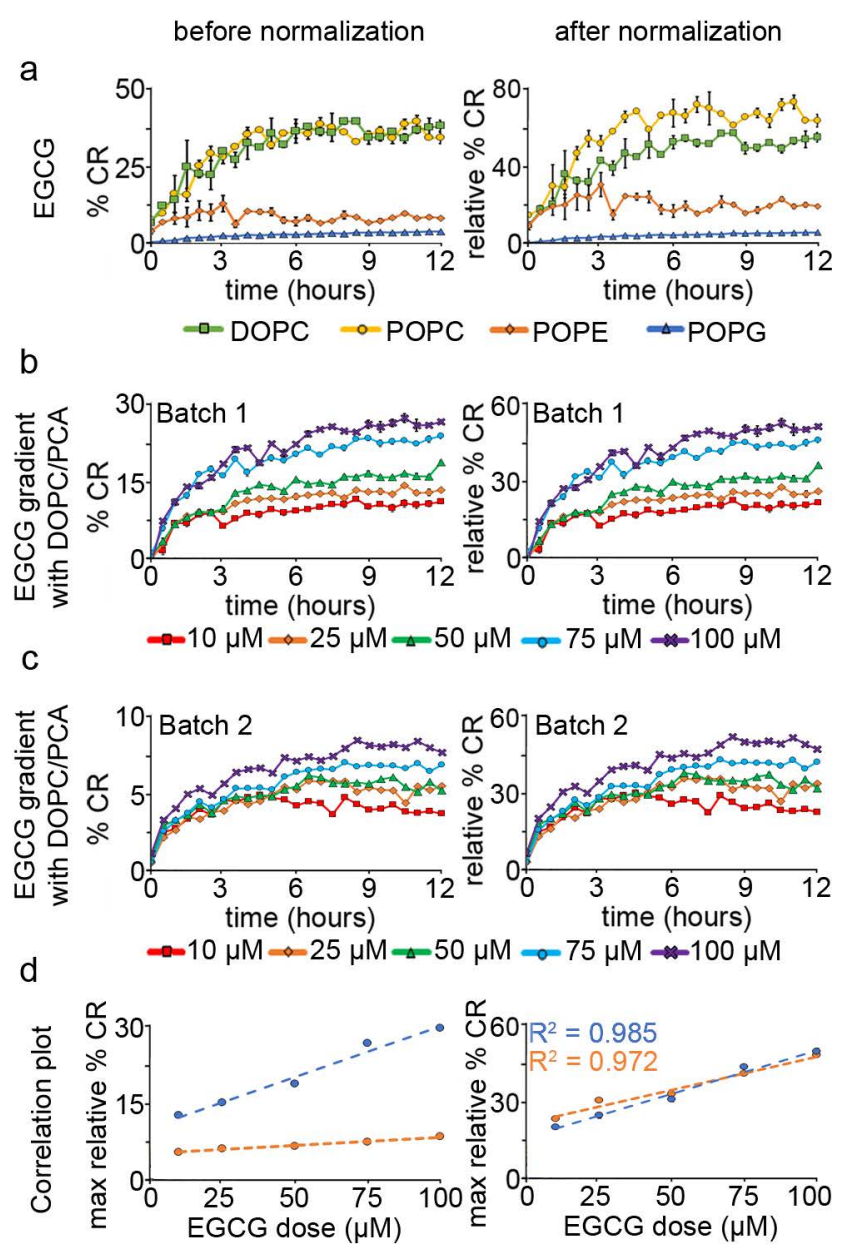

$\rightarrow$ Batch $1 \rightarrow$ Batch 2

e
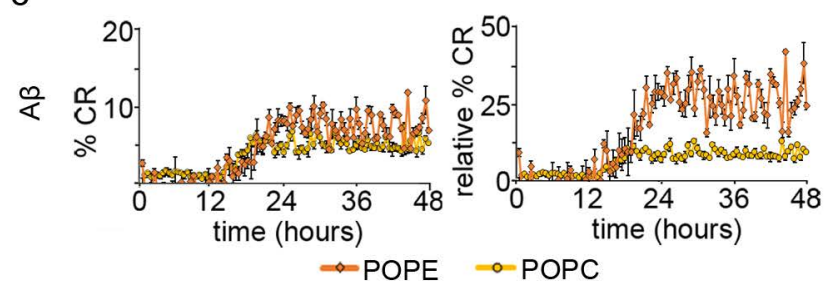

Figure 2.3 PDA/lipid vesicles exhibit a colorimetric response upon exposure to EGCG and A $\boldsymbol{\beta}$ peptide. The left column presents data before normalization, and the right column presents data after normalization with a $100 \mu \mathrm{M} \mathrm{NaOH}$ standard. (a) \% CR kinetic curves for different PDA/lipid systems (DOPC, POPC, POPE, and POPG) exposed to EGCG. (b-c) EGCG dose dependency \%CR kinetic curves for two distinct batches of DOPC/PDA vesicles. (d) Maximum relative $\% \mathrm{CR}$ induced in two distinct batches of DOPC/PDA vesicles as a function of EGCG dose. (e) \%CR kinetic curves for different PDA/lipid systems exposed to $\mathrm{A} \beta$ before and after normalization with a $100 \mu \mathrm{M} \mathrm{NaOH}$ standard. Data points are the average of independent triplicates of each condition, and error bars represent standard error.

Interaction with lipid membranes has a large impact on the pharmacokinetic properties of different molecules, such as the transport, distribution, and accumulation. To determine the accuracy of PDA assays in measuring relative membrane affinity of molecules, the four PDA/lipid vesicle systems (DOPC, POPC, POPE, and POPG) were exposed to (-) epigallocatechin-3-gallate (EGCG) (Fig. 2.3a). EGCG is an abundant catechin in green tea, 
and its bioactivity has been extensively studied for potential therapeutic applications, ranging from being an effective cancer chemopreventative ${ }^{32}$ to an amyloid formation inhibitor..$^{22,33-35}$ EGCG stocks were prepped in DMSO at a high enough concentration so that DMSO comprised $1 \%$ of the final volume within a well, which does not elicit a CR (Fig. 2.4a). Exposure to EGCG $(100 \mu \mathrm{M})$ elicited a CR in all four lipid systems to varying degrees (Fig. 2.3a). Before normalization, the CR elicited by EGCG was similar for POPC and DOPC (maximum \% CR $\sim 38 \%$ ). EGCG interacted with POPE vesicles (maximum \% CR 9\%) and POPG vesicles (maximum \% CR 4\%) to a lesser extent. Upon normalization, it is apparent that EGCG has a higher affinity for POPC compared with DOPC (71.91\% versus 56.93\%). The trend with POPE and POPG vesicles was unchanged. As an additional control, PDA vesicles of all four lipid systems were exposed to a small molecule, riluzole, that does not interact with lipid membranes, and no CR was elicited (Fig. 2.4b). The relative lipid binding affinity for EGCG for the different lipid systems after normalization is consistent with literature reports using other methods. EGCG readily interacts with lipid membranes containing PC lipids. ${ }^{36-38}$ Specifically, EGCG damages PC liposomes, resulting in membrane leakage. ${ }^{39}$ This damage of PC lipids membranes would invoke a mechanical stress that would elicit the CR in PDA vesicles observed with the two PC lipid systems. Furthermore, X-ray lamellar diffraction patterns of POPC and DOPC bilayers indicate that EGCG more strongly disrupts POPC bilayers. ${ }^{40}$ This difference was only apparent here after normalization of the PDA assays. The decreased $\% \mathrm{CR}$ associated with exposure of POPE to EGCG in comparison to PC lipids is consistent with molecular dynamic simulations demonstrating a decreased likelihood of EGCG forming bonds with POPE. ${ }^{41}$ Finally, anionic lipids further reduce the affinity of EGCG for lipids as determined by a reduced ability of EGCG to quench NPN-fluorescence, ${ }^{39}$ which correlates with POPG inducing the smallest relative $\%$ 
CR. In addition, two different batches of DOPC/PDA vesicles with varying sensitivities were exposed to EGCG to determine reproducibility of a dose response (10-100 $\mu \mathrm{M}$, Fig. 2.3b-c).

With larger EGCG dose, \% CR increased. The maximum relative \% CR linearly correlated with EGCG dose; however, the relationship varied between batches (Fig. 2.3d). This batch-to-batch variability was significantly reduced by normalization.

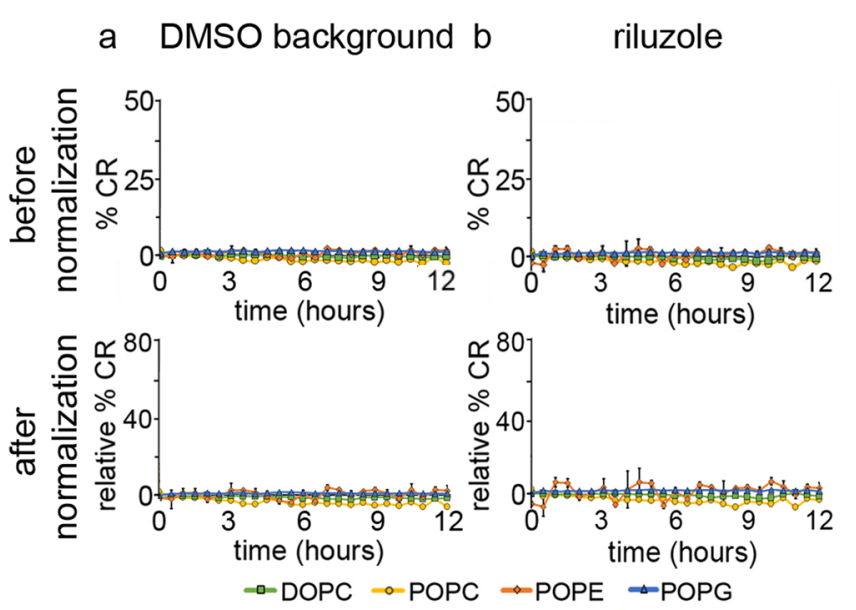

Figure 2.4 DMSO and riluzole do not elicit a colorimetric response. PDA/lipid vesicle response to the (a) DMSO or (b) riluzole. The top row presents data before normalization, and the bottom row presents data after normalization with a $100 \mu \mathrm{M} \mathrm{NaOH}$ standard. Data points are the average of independent triplicates of each condition, and error bars represent standard error.

Next, the $\beta$-amyloid peptide (A $\beta$ ) peptide was used as a model system to investigate the applicability of normalized PDA assays to probe peptide/membrane interactions (Fig. 2.3e). A $\beta$ aggregation is a hallmark of Alzheimer's Disease (AD). Numerous toxic mechanisms involve A $\beta$ 's ability to bind cellular membranes, and these interactions are heavily influenced by membrane composition. ${ }^{42,43}$ As a result, understanding the relative affinity of $A \beta$ for different lipid components can provided insight into a variety of toxic mechanisms associated with $A \beta$. For this study, only POPC and POPE PDA/lipid vesicles were used as comparable, unambiguous experiments in the literature were available. Each of these lipids are electrostatically neutral but have different physicochemical properties that are largely influenced by the size of the lipid headgroups, such as fluidity and area per lipid. PDA/POPC or PDA/POPE vesicles systems were 
exposed to $\mathrm{A} \beta_{40}(20 \mu \mathrm{M})$, and $\% \mathrm{CR}$ was measured every 30 min for $48 \mathrm{~h}$. Parallel $\mathrm{NaOH}(100$ $\mathrm{mM}$ ) standard experiments were performed simultaneously and used for normalization. $A \beta$ did not induce a CR with either POPC or POPE for $\sim 12 \mathrm{~h}$, then the $\% \mathrm{CR}$ steadily increased for several hours in both systems, reaching a steady-state response at $\sim 18 \mathrm{~h}$ and $21 \mathrm{~h}$ for POPC and POPE respectively. Prior to normalization, the steady-state \% CR was larger for POPE; however, this was not statistically significant. After normalization, $A \beta$ had a larger and statistically significant $(\mathrm{p}<0.01)$ interaction with POPE in comparison with POPC, consistent with previous observations of $A \beta$ interactions with POPC or POPE. ${ }^{44,45} \mathrm{~A} \beta$ protofibrils exhibit stronger electrostatic interactions with POPE than POPC due to the larger POPC headgroup restricting the interaction between $\mathrm{A} \beta$ and the phosphate of the lipid head group,${ }^{44}$ and an overall decreased affinity of $\mathrm{A} \beta$ for POPC compared to POPE has been observed with MD simulations. ${ }^{45}$ The consistency with literature reports suggest that the normalized PDA assays accurately reflect the relative interaction of $\mathrm{A} \beta 40$ with these different lipid systems.

\subsection{Conclusion}

PDA lipid binding assays are a simple, cheap, and accurate method of determining relative membrane binding affinity that can be used in high-throughput experimentation. However, there are complications associated with batch-to-batch reproducibility and in comparing across different lipid systems. Here, a simple normalization procedure was demonstrated that addresses both issues. A CR was induced in PDA/lipid vesicles in a dose dependent manner upon exposure to $\mathrm{NaOH}$, and the magnitude of the $\% \mathrm{CR}$ was linear within a working range across four different lipid systems (DOPC, POPC, POPE, and POPG). Normalization of the PDA assays by making signals relative to the maximum achievable CR for a lipid system through the use of an $\mathrm{NaOH}$ standard resulted in significantly lowered batch-to- 
batch and between-lipid RSD values. Standardization of PDA assays with $\mathrm{NaOH}$ was then used to determine the relative affinity of a model small molecule (EGCG) and peptide (A $\beta)$ with different lipids. In all cases, results of the normalized PDA assay aligned with previous literature on the relative membrane affinity of $\mathrm{EGCG}^{39-41}$ and $\mathrm{A} \beta .^{44,45}$ With this normalization of PDA lipid binding assays, meaningful comparisons can be made between batches and lipid systems, enabling a variety of experiments to determine relative membrane affinities of different molecules, pharmaceuticals, and peptides.

\subsection{References}

1. Shimizu, T. Lipid Mediators in Health and Disease: Enzymes and Receptors as Therapeutic Targets for the Regulation of Immunity and Inflammation. Annu. Rev. Pharmacol. Toxicol. 49, 123-150 (2009).

2. Kerns, E. H. High throughput physicochemical profiling for drug discovery. J. Pharm. Sci. 90, 1838-1858 (2001).

3. Thukral, L. et al. The Molecular Mechanism Underlying Recruitment and Insertion of LipidAnchored LC3 Protein into Membranes. Biophys. J. 109, 2067-2078 (2015).

4. Nakamura, M. T., Yudell, B. E. \& Loor, J. J. Regulation of energy metabolism by long-chain fatty acids. Prog. Lipid Res. 53, 124-144 (2014).

5. Escribá, P. V. Membrane-lipid therapy: A historical perspective of membrane-targeted therapies - From lipid bilayer structure to the pathophysiological regulation of cells. Biochim. Biophys. Acta, Biomembr. 1859, 1493-1506 (2017).

6. van Meer, G. Cellular lipidomics. EMBO J. 24, 3159-3165 (2005).

7. Yetukuri, L., Ekroos, K., Vidal-Puig, A. \& Orešič, M. Informatics and computational strategies for the study of lipids. Mol. Biosyst. 4, 121-127 (2008).

8. Bloom, M., Evans, E. \& Mouritsen, O. G. Physical properties of the fluid lipid-bilayer component of cell membranes: a perspective. Q. Rev. Biophys. 24, 293-397 (1991).

9. Casares, D., Escribá, P. V. \& Rosselló, C. A. Membrane Lipid Composition: Effect on Membrane and Organelle Structure, Function and Compartmentalization and Therapeutic Avenues. Int. J. Mol. Sci. 20, (2019).

10. Goñi, F. M. The basic structure and dynamics of cell membranes: An update of the SingerNicolson model. Biochim. Biophys. Acta. - Biomembr. 1838, 1467-1476 (2014). 
11. White, S. H. \& Wimley, W. C. Hydrophobic interactions of peptides with membrane interfaces. Biochim. Biophys. Acta. - Biomembr. 1376, 339-352 (1998).

12. Krämer, S. D., Braun, A., Jakits-Deiser, C. \& Wunderli-Allenspach, H. Towards the Predictability of Drug-Lipid Membrane Interactions: The $\mathrm{pH}-$ Dependent Affinity of Propranolol to Phosphatidylinositol Containing Liposomes. Pharm. Res. 15, 739-744 (1998).

13. Gollnick, P. TRAP-RNA Interactions Involved in Regulating Transcription Attenuation of the Bacillus subtilis trp Operon. Methods Enzymol. vol. 371 405-418 (Elsevier, 2003).

14. Krause, E., Dathe, M., Wieprecht, T.\& Bienert, M. Noncovalent immobilized artificial membrane chromatography, an improved method for describing peptide-lipid bilayer interactions. J. Chromatogr. A 849, 125-133 (1999).

15. Lee, T.-H., Mozsolits, H. \& Aguilar, M.-I. Measurement of the affinity of melittin for zwitterionic and anionic membranes using immobilized lipid biosensors. J. Pept. Res. 58, 464-476 (2001).

16. Perkel, J. Surface Plasmon Resonance (SPR) and Other Options for Label-free Detection. http://www.biocompare.com/Editorial-Articles/41803-Surface-Plasmon-Resonance-SPRand-Other-Options-for-Label-free-Detection/.

17. Perkel, J. Microplate Readers: Many Options for Multiple Applications. http://www.biocompare.com/Editorial-Articles/137081-Microplate-Readers-Many-Optionsfor-Multiple-Applications/.

18. Ryu, S., Yoo, I., Song, S., Yoon, B. \& Kim, J.-M. A thermoresponsive fluorogenic conjugated polymer for a temperature sensor in microfluidic devices. J. Am. Chem. Soc. 131, 3800-3801 (2009).

19. Kew, S. J. \& Hall, E. A. H. pH response of carboxy-terminated colorimetric polydiacetylene vesicles. Anal. Chem. 78, 2231-2238 (2006).

20. Gao, X. et al. Cholesterol Modifies Huntingtin Binding to, Disruption of, and Aggregation on Lipid Membranes. Biochemistry 55, 92-102 (2016).

21. Chaibva, M. et al. Acetylation within the First 17 Residues of Huntingtin Exon 1 Alters Aggregation and Lipid Binding. Biophys. J. 111, 349-362 (2016).

22. Beasley, M. et al. Lipid membranes influence the ability of small molecules to inhibit huntingtin fibrillization. Biochemistry (2019) doi:10.1021/acs.biochem.9b00739.

23. Kolusheva, S., Friedman, J., Angel, I. \& Jelinek, R. Membrane Interactions and Metal Ion Effects on Bilayer Permeation of the Lipophilic Ion Modulator DP-109. Biochemistry 44, 12077-12085 (2005).

24. A quantitative method for the measurement of membrane affinity by polydiacetylene-based colorimetric assay. Anal. Biochem. 420, 171-176 (2012). 
25. Kolusheva, S., Wachtel, E. \& Jelinek, R. Biomimetic lipid/polymer colorimetric membranes molecular and cooperative properties. J. Lipid Res. 44, 65-71 (2003).

26. Kolusheva, S., Boyer, L. \& Jelinek, R. A colorimetric assay for rapid screening of antimicrobial peptides. Nat. Biotechnol. 18, 225-227 (2000).

27. Yadav, M. K., Kumar, V., Singh, B. \& Tiwari, S. K. Phospholipid/Polydiacetylene VesicleBased Colorimetric Assay for High-Throughput Screening of Bacteriocins and Halocins. Appl. Biochem. Biotechnol. 182, 142-154 (2017).

28. Katz, M., Ben-Shlush, I., Kolusheva, S. \& Jelinek, R. Rapid Colorimetric Screening of Drug Interaction and Penetration Through Lipid Barriers. Pharm. Res. 23, 580-588 (2006).

29. Katz, M. et al. Lipid binding and membrane penetration of polymyxin B derivatives studied in a biomimetic vesicle system. Biochem. J. 375, 405-413 (2003).

30. Halevy, R., Rozek, A., Kolusheva, S., Hancock, R. E. W. \& Jelinek, R. Membrane binding and permeation by indolicidin analogs studied by a biomimetic lipid/polydiacetylene vesicle assay. Peptides 24, 1753-1761 (2003).

31. Song, J., Cheng, Q., Kopta, S. \& Stevens, R. C. Modulating Artificial Membrane Morphology: $\mathrm{pH}-$ Induced Chromatic Transition and Nanostructural Transformation of a Bolaamphiphilic Conjugated Polymer from Blue Helical Ribbons to Red Nanofibers. J. Am. Chem. Soc. 123, 3205-3213 (2001).

32. Du, G.-J. et al. Epigallocatechin Gallate (EGCG) Is the Most Effective Cancer Chemopreventive Polyphenol in Green Tea. Nutrients 4, 1679-1691 (2012).

33. Bieschke, J. et al. EGCG remodels mature alpha-synuclein and amyloid-beta fibrils and reduces cellular toxicity. Proc. Natl. Acad. Sci. U. S. A. 107, 7710-7715 (2010).

34. Palhano, F. L., Lee, J., Grimster, N. P. \& Kelly, J. W. Toward the Molecular Mechanism(s) by which EGCG Treatment Remodels Mature Amyloid Fibrils. J. Am. Chem. Soc. 135, 7503-7510 (2013).

35. Xu, Y. et al. Epigallocatechin Gallate (EGCG) Inhibits Alpha-Synuclein Aggregation: A Potential Agent for Parkinson's Disease. Neurochem. Res. 41, 2788-2796 (2016).

36. Hashimoto, T., Kumazawa, S., Nanjo, F., Hara, Y. \& Nakayama, T. Interaction of Tea Catechins with Lipid Bilayers Investigated with Liposome Systems. Biosci. Biotechnol. Biochem. 63, 2252-2255 (1999).

37. Kajiya, K., Kumazawa, S. \& Nakayama, T. Steric Effects on Interaction of Tea Catechins with Lipid Bilayers. Biosci. Biotechnol. Biochem. 65, 2638-2643 (2001).

38. Kajiya, K. et al. Relationship between Antibacterial Activity of (+)-Catechin Derivatives and Their Interaction with a Model Membrane. J. Agric. Food Chem. 52, 1514-1519 (2004). 
39. Ikigai, H., Nakae, T., Hara, Y. \& Shimamura, T. Bactericidal catechins damage the lipid bilayer. Biochim. Biophys. Acta BBA - Biomembr. 1147, 132-136 (1993).

40. Sun, Y., Hung, W.-C., Chen, F.-Y., Lee, C.-C. \& Huang, H. W. Interaction of Tea Catechin (-)-Epigallocatechin Gallate with Lipid Bilayers. Biophys. J. 96, 1026-1035 (2009).

41. Villalaín, J. Epigallocatechin-3-gallate location and interaction with late endosomal and plasma membrane model membranes by molecular dynamics. J. Biomol. Struct. Dyn. 37, 3122-3134 (2019).

42. Hertel, C. et al. Inhibition of the electrostatic interaction between $\beta$-amyloid peptide and membranes prevents $\beta$-amyloid-induced toxicity. Proc. Natl. Acad. Sci. U. S. A. 94, 94129416 (1997).

43. Kourie, J. I., Culverson, A. L., Farrelly, P. V., Henry, C. L. \& Laohachai, K. N. Heterogeneous amyloid-formed ion channels as a common cytotoxic mechanism. Cell Biochem. Biophys. 36, 191-207 (2002).

44. Tofoleanu, F., Brooks, B. R. \& Buchete, N.-V. Modulation of Alzheimer's A $\beta$ protofilament-membrane interactions by lipid headgroups. ACS Chem. Neurosci. 6, 446-455 (2015).

45. Tofoleanu, F. \& Buchete, N.-V. Molecular Interactions of Alzheimer's A $\beta$ Protofilaments with Lipid Membranes. J. Mol. Biol. 421, 572-586 (2012). 


\section{Lipid membranes influence the ability of small molecules to inhibit huntingtin fibrillization ${ }^{1}$}

Several diseases, including Alzheimer's disease, Parkinson's disease, and Huntington's disease (HD), are associated with specific proteins aggregating and depositing within tissues and/or cellular compartments. The aggregation of these proteins is characterized by the formation of extended, $\beta$-sheet rich fibrils, termed amyloid. In addition, a variety of other aggregate species also form, including oligomers and protofibrils. Specifically, HD is caused by the aggregation of the huntingtin (htt) protein that contains an expanded polyglutamine domain. Due to the link between protein aggregation and disease, small molecule aggregation inhibitors have been pursued as potential therapeutic agents. Two such small molecules are epigallocatechin 3-gallate (EGCG) and curcumin, both of which inhibit the fibril formation of several amyloid-forming proteins. However, amyloid formation is a complex process that is strongly influenced by the protein's environment, leading to distinct aggregation pathways. Thus, changes in the protein's environment may alter the effectiveness of aggregation inhibitors. A well-known modulator of amyloid formation is lipid membranes. Here, we investigated if the presence of lipid vesicles altered the ability of EGCG or curcumin to modulate htt aggregation and influence the interaction of htt with lipid membranes. The presence of 1-palmitoyl-2-oleoyl-glycero-3-phosphocholine or total brain lipid extract vesicles prevented the curcumin from inhibiting htt fibril formation. In contrast, EGCG's inhibition of htt fibril formation persisted in the presence of lipids. Collectively, these

${ }^{1}$ Reprinted with permission from Beasley, M. et al. Lipid membranes influence the ability of small molecules to inhibit huntingtin fibrillization. Biochemistry 58, 4361-4373. Copyright 2019 American Chemical Society 
results highlight the complexity of htt aggregation and demonstrate that the presence of lipid membranes is a key modifier of the ability of small molecules to inhibit htt fibril formation.

\subsection{Introduction}

The polymerization of specific proteins into fibril aggregates with an underlying cross- $\beta$ structure, generically referred to as amyloid, is associated with a broad range of diseases. For example, aggregation of the amyloid- $\beta$ peptide $(\mathrm{A} \beta)$ and tau are associated with Alzheimer's disease; $\alpha$-synuclein ( $\alpha$-syn, UniProtKB P37840) is implicated in Parkinson's disease; aggregates of islet amyloid polypeptide (IAPP, UniProtKB P10997) form in diabetes mellitus type 2; and mutated forms of the huntingtin protein (htt, UniProtKB P42858) with an expanded polyglutamine (polyQ) stretch underlie Huntington's disease (HD). Amyloid formation is a complex, heterogeneous process that involves a number of intermediate aggregate species, including a variety of oligomers, protofibrils, and amorphous aggregates. ${ }^{1-3}$ In particular, oligomers have been implicated as prominent toxic species. ${ }^{4-6}$ The aggregation process for any given amyloid-forming protein is highly dependent on its environment. For example, changes in $\mathrm{pH},{ }^{7,8}$ metal ions, ${ }^{9}$ and preparatory protocols ${ }^{10}$ influence aggregate structure and morphology. In particular, HD is caused by an expanded polyglutamine (polyQ) domain that must surpass a critical threshold of $\sim 35$ repeat units, ${ }^{11-13}$ and the extent of htt fibrillization correlates directly with increasing polyQ length. ${ }^{14-16} \mathrm{Htt}$ can form a heterogeneous mixture of oligomers ${ }^{15,17-19}$ that have been observed in a variety of HD models. ${ }^{20-24}$

Due to the apparent role of protein aggregation in amyloid diseases, a number of small molecules that inhibit or modify amyloid formation have been identified. ${ }^{25,26}$ Two specific 
polyphenols, (-)-epigallocatechin-3-gallate (EGCG) and curcumin, have been extensively studied due to their ability to generically inhibit amyloid formation. EGCG, a compound commonly found in green tea, reduces aggregation and cytotoxicity of $A \beta^{27-30}, \alpha$-syn, ${ }^{27,31,32}$ and tau. ${ }^{33}$ For htt, EGCG decreases the formation of toxic htt aggregates in both yeast and Drosophila models. ${ }^{34}$ Curcumin, an abundant molecule in the spice turmeric, inhibits fibril formation of $A \beta$ and $\alpha-S y n .{ }^{35-37}$ With regards to htt, the ability of curcumin to inhibit fibril formation is less clear, as it has been reported to both promote ${ }^{38}$ and inhibit htt fibril formation. ${ }^{39}$ This apparent contradiction may lie in the ability of other cellular factors to influence htt aggregation and the interaction between htt and curcumin.

With the notion that the cellular environment may influence the efficacy of small molecule inhibitors of amyloid formation, it becomes important to understand how specific cellular components influence the ability of small molecules to modify aggregation. Lipid membranes are known modulators of aggregation as altered aggregation in the presence of membranes, compared to bulk solution, has been observed for multiple amyloids, including htt, ${ }^{40-43} \mathrm{~A} \beta,{ }^{44-47}$ and $\alpha$-syn. ${ }^{48,49}$ The presence of cellular membranes comprised of lipid bilayers also alters amyloid aggregation by nucleating aggregation or stabilizing certain aggregate species and morphologies. ${ }^{50}$ Specifically with regards to HD, mutant htt shows increased binding affinity for phospholipids over non-mutated htt and can also disrupt the structural integrity of phospholipid bilayers. ${ }^{51-53}$ EGCG has been shown to inhibit permeabilization of model membranes and mitochondrial membranes by $\mathrm{A} \beta$ and $\alpha$-syn aggregates ${ }^{54-56}$ EGCG also interacts with and binds to membranes themselves, but significantly decreases membrane disruption capabilities of $\mathrm{A} \beta$ when pre-incubated with the peptide. ${ }^{57}$ 
To determine the effect these small molecules have on htt aggregation in membranous environments, we investigated if the presence of lipid altered the ability of either EGCG or curcumin to modulate aggregation and influence htt lipid binding. We found that the presence of either pure or physiological membrane systems interfered with the ability of curcumin to decrease htt fibril formation, while EGCG's inhibition of fibril formation persisted in the presence of lipids. Our studies suggest that the interaction between htt and curcumin is largely altered by cellular environment, while EGCG led to a propensity for the formation of amorphous aggregates regardless of the interaction with membrane systems.

\subsection{Materials and Methods}

\subsubsection{Purification of GST-htt-exon1 fusion protein}

Glutathione S-transferase (GST)-htt-exon1(46Q) fusion proteins were expressed and purified as previously described. ${ }^{58}$ In short, the GST-htt fusion proteins were expressed by induction in Escherichia coli with isopropyl-thio-galactopyranoside (IPTG) for four hours at 30 ${ }^{\circ} \mathrm{C}$. The cells were lysed using both lysozyme $(0.5 \mathrm{mg} / \mathrm{mL})$ and sonication, and the fusion proteins were purified by liquid chromatography (BioRad LPLC) using a $5 \mathrm{~mL}$ GST affinity column. Fractions were analyzed using gel electrophoresis to verify the presence and purity of htt. Protein concentration was determined using Coomassie Bradford reagent. Prior to any assay, fusion proteins were subjected to high speed centrifugation at $20,000 \mathrm{~g}$ for 45 minutes at $4{ }^{\circ} \mathrm{C}$ to remove pre-existing aggregates. To initiate aggregation for experimentation, the fusion proteins were incubated with Factor Xa (Promega, Madison, WI) to cleave the GST tag. All experiments were performed in buffer A (50mM Tris- $\mathrm{HCl}, \mathrm{pH} 7,150 \mathrm{mM} \mathrm{NaCl})$. 


\subsubsection{Preparation of lipid vesicles}

Lipid vesicles of 1-palmitoyl-2-oleoyl-glycero-3-phosphocholine (POPC) or total brain lipid extract (TBLE) were prepared by rehydrating the lipid film in buffer A. Lipid solutions were then subjected to 10 freeze/thaw cycles using liquid nitrogen followed by bath sonication for 30 minutes. The size and polydispersity of lipid vesicles were measured with dynamic light scattering (NanoBrook 90plus Particle Size Analyzer, Brookhaven Instruments) to verify the formation of large unilamellar vesicles (LUVs). The mean and standard deviation of vesicle sizes were determined by assuming a lognormal distribution of the DLS data.

\subsubsection{Thioflavin $\mathbf{T}$ aggregation assays}

Purified htt-exon1(46Q) was diluted to a final concentration of $20 \mu \mathrm{M}$ in the presence of $125 \mu \mathrm{M}$ Thioflavin T (ThT, Sigma-Aldrich, St. Louis, MO) and the desired small molecule (20 $\mu \mathrm{M}$ or $100 \mu \mathrm{M})$. In experiments when lipid was used, the lipid concentration was $400 \mu \mathrm{M}$, resulting in a 20:1 lipid:protein ratio. Reactions were run in black Costar 96-well plates with clear flat bottom and the ThT fluorescence was monitored using a SpectraMax M2 microplate reader. Experiments were run at $37^{\circ} \mathrm{C}$ with $440 \mathrm{~nm}$ excitation and $484 \mathrm{~nm}$ emission, with readings every 5 minutes for 18 hours. The initial rate of each ThT plot was calculated over a period of three hours, starting at the point where the fluorescence signal was larger than the set threshold. The threshold was set at $10 \%$ of the maximum signal obtained for each condition; this ensures that the data has made a significant increase from the baseline and avoids rate calculations starting in the noise of the lag phase. These initial aggregation rates were normalized to the htt control $(100 \%)$ for ease of comparison. The relative maximum fluorescence was 
determined by finding the maximum fluorescence intensity for each condition and normalizing them to the maximum fluorescence intensity of the htt control $(100 \%)$.

\subsubsection{Atomic force microscopy (AFM)}

Incubations of htt-exon1(46Q) $(20 \mu \mathrm{M})$ in the presence and absence of small molecule aggregation inhibitors $(100 \mu \mathrm{M})$ were maintained at $37^{\circ} \mathrm{C}$ and $1400 \mathrm{rpm}$ using an orbital mixer. Control incubations of small molecule aggregation inhibitors $(100 \mu \mathrm{M})$ alone were also used. In addition, incubations performed with lipid vesicles were done at a lipid to peptide ratio of 20:1. At various time points, $2 \mu \mathrm{L}$ aliquots of each condition were deposited onto freshly cleaved mica (Ted Pella Inc., Redding, CA) for 1 minute, washed with $200 \mu \mathrm{L}$ of ultra-pure water, and dried using a gentle stream of clean air. Images were collected using a Nanoscope V Multi-Mode scanning probe microscope (VEECO) equipped with a closed loop vertical engage J-scanner. Silicon-oxide cantilevers with a nominal spring constant of $40 \mathrm{~N} / \mathrm{m}$ and a resonance frequency of $300 \mathrm{kHz}$ were used. Scan rates were set between 1 and $2 \mathrm{~Hz}$ with cantilever drive frequencies at $10 \%$ of resonance. All images were then analyzed using the MATLAB image processing toolbox (MathWorks) as previously described. ${ }^{59,60}$

\subsubsection{Vesicle-binding assay}

To measure the interaction between the htt and lipid vesicles, a polydiacetylene (PDA) lipid binding assay was performed using previously reported protocols. ${ }^{61,62}$ In short, diacetylene monomers of 10,12-tricosadiynoic acid (GFS Chemicals, Columbus, OH) and the lipid system of choice were mixed at a 2:3 molar ratio in a 4:1 chloroform/ethanol solution. The solution was evaporated off under a gentle stream of nitrogen, and then the resulting dry films were reconstituted in buffer $\mathrm{A}$ at $70^{\circ} \mathrm{C}$. Lipid solutions were then sonicated at $100 \mathrm{~W}$ for 10 minutes 
using a sonic dismembrator (FisherSci) before being stored overnight at $4{ }^{\circ} \mathrm{C}$ to allow the selfassembly of vesicles. The next day, lipid mixtures were irradiated at $254 \mathrm{~nm}$ for 15 minutes with constant stirring to polymerize the 10,12-tricosadiynoic acid and result in a dark, royal blue solution. Experiments were performed in triplicate in a 96-well format, with the colorimetric response being recorded every 5 minutes for 18 hours using a SpectraMax M2 microplate reader. The negative control included equal ratios of PDA/lipid solution and buffer, while the positive control included saturated $\mathrm{NaOH}(\mathrm{pH}=12)$ to create a range of colorimetric response for each lipid system. Polymerized vesicles were exposed to htt at a final concentration of $20 \mu \mathrm{M}$ and small molecule concentrations of $100 \mu \mathrm{M}$. The PB, defined by $\mathrm{A}_{\text {blue }} /\left(\mathrm{A}_{\text {blue }}+\mathrm{A}_{\mathrm{red}}\right)$ was calculated for the control $\left(\mathrm{PB}_{0}\right)$ and each sample condition $(\mathrm{PB})$. The percent colorimetric response $(\% \mathrm{CR})$ indicates the extent of insertion or disruption of the lipid membrane by htt and was calculated using the following equation:

$$
\% C R=\left(\frac{P B_{0}-P B}{P B_{0}}\right) \times 100
$$

Data was normalized to the maximum colorimetric response for each system, which was found by incubating the PDA/lipid solutions with 1:1 $1 \mathrm{M} \mathrm{NaOH}$.

\subsection{Results}

\subsubsection{Curcumin and EGCG inhibit htt fibrillization}

All experiments in this study were performed with a mutant htt fragment that expresses exon 1 with 46Q (htt-exon1(46Q)), which was purified from Escherichia coli as a soluble fusion with glutathione S-transferase (GST). Cleavage of the GST moiety with factor Xa released the htt-exon1(46Q) fragment, initiating aggregation. To determine the relative ability of curcumin 
and EGCG to inhibit htt fibril formation, ThT aggregation assays were performed with httexon1(46Q) and varying ratios of the small molecules (1:1 and 5:1 small molecule to htt; Fig. 3.1a-b). All relevant controls were measured and subtracted from the curves to show only the fluorescence signal from the htt aggregation (Fig. 3.2), and multiple independent experiments were performed (Fig. 3.3). For the initial relative aggregation rate, curcumin slowed aggregation to $50 \%$ and $35 \%$ of the control rate for 1:1 and 5:1 treatments respectively (Fig. 3.1c). At a 1:1 ratio, curcumin reduced the overall ThT signal to $62 \%$ of the control signal, and increasing the dose of curcumin to 5:1 resulted in an additional reduction to $48 \%$ of the control signal (Fig. 3.1d). EGCG was more effective in reducing apparent fibril formation, as the relative initial aggregation rate was reduced to a larger extent (31\% and $10 \%$ of the control rate for $1: 1$ and $5: 1$ treatments respectively; Fig. 3.1c). EGCG reduced the maximum ThT fluorescence signal to $32 \%$ and $24 \%$ of the control signal for the 1:1 and 5:1 doses respectively (Fig. 3.1d). While ThT fluorescence is traditionally considered to track fibril formation, ThT signals are not always indicative of fibril formation and does not provide information about the formation of other aggregate species. As a result, further experimentation is required to verify aggregate inhibition.
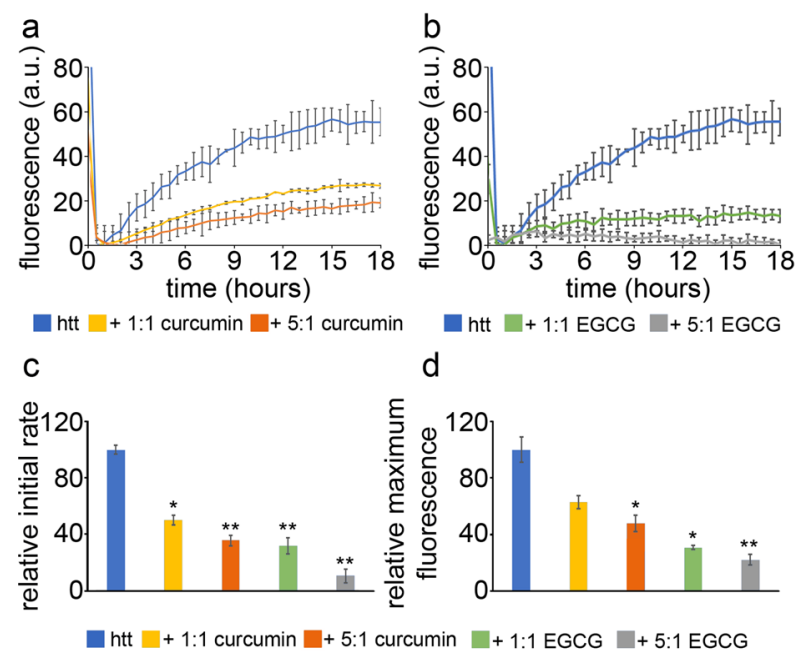

Figure 3.1 Curcumin and EGCG decrease htt fibrillization. ThT aggregation assays for htt-exon 1(46Q) in the presence of (a) curcumin or (b) EGCG. Htt-exon 1(46Q) concentrationwas $20 \mu \mathrm{M}$. (c) The initial rate of aggregation and (d) the relative maximum fluorescence were determined with respect to the htt control. Normalization was performed with respect to the htt control. Analysis shown in (c) and (d) were determined as averages over all trials (shown in Fig. 3.3). Error bars are provided for every $6^{\text {th }}$ datapoint $(30 \mathrm{~min})$ and represent the standard error of the mean. * represents a $\mathrm{p}$ value $<0.05$ and $* *$ represents a $\mathrm{p}$ value $<0.01$. 

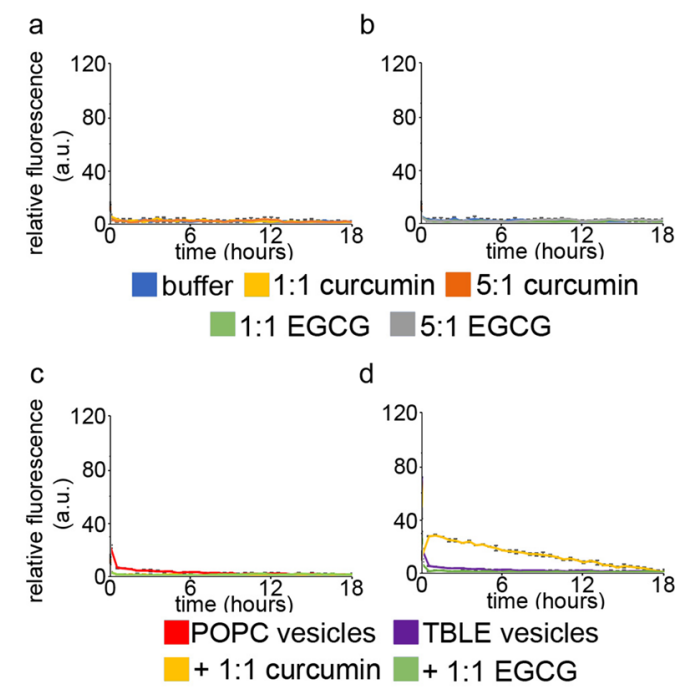

Figure 3.2 Small molecules alone or with lipid do not result in a ThT signal. ThT fluorescence assay controls of (a) curcumin and (b) EGCG alone, as well as controls of the small molecules in the presence of (c) POPC or (d) TBLE vesicles.
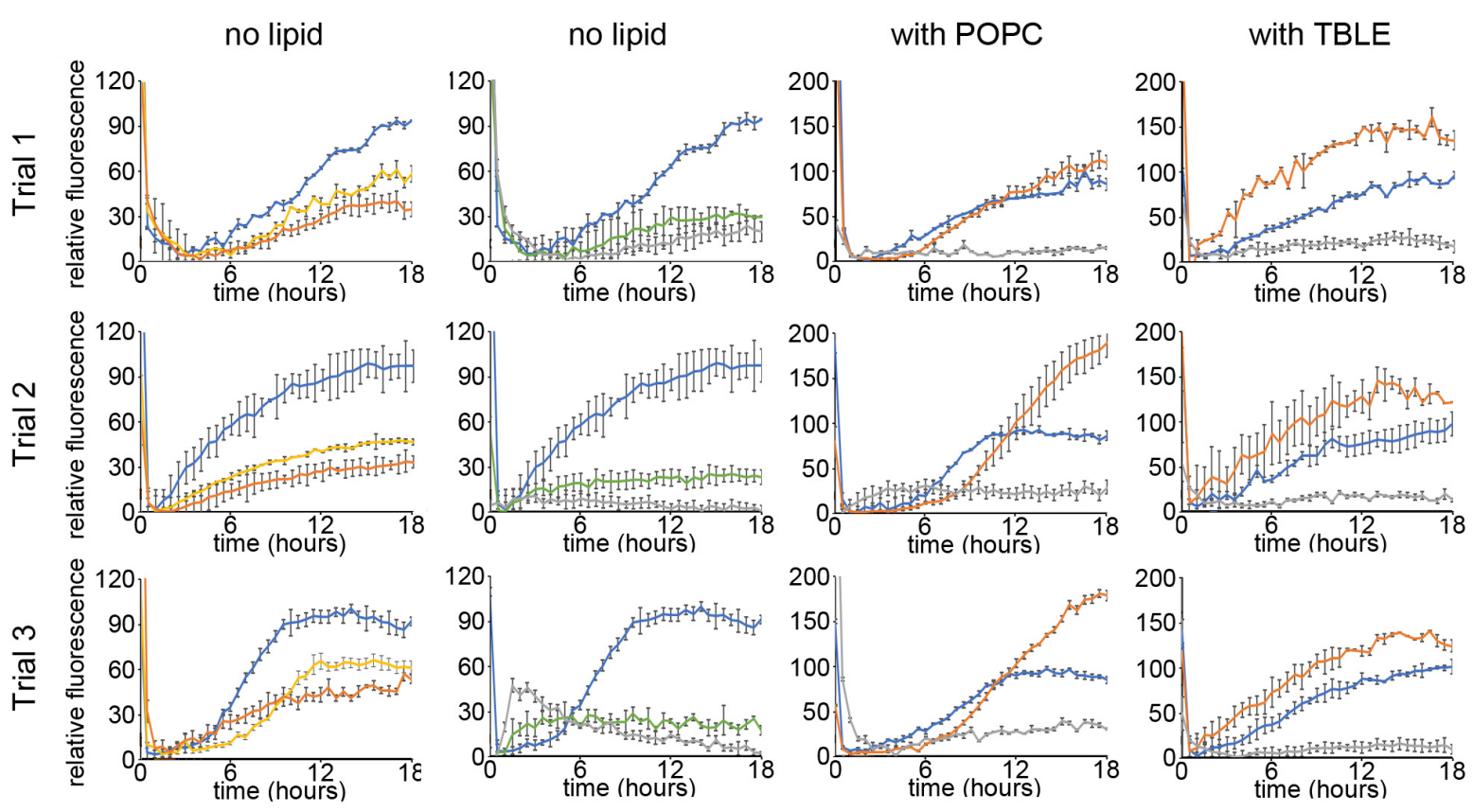

htt $\square+1: 1$ curcumin $\square+5: 1$ curcumin

$+1: 1$ EGCG $+5: 1$ EGCG

Figure 3.3 The effect of small molecules on htt fibrillization is consistent across all experiments. Individual trials of ThT assays for $20 \mu \mathrm{M}$ htt (blue) in the presence of 1:1 curcumin (yellow), 5:1 curcumin (orange), 1:1 EGCG (green), or 5:1 EGCG (gray). All ratios are provided as small molecule to htt protein. ThT curves were performed without lipids or in the presence of either POPC or TBLE vesicles. Three independent plates were run for each condition. 
To further establish the inhibition of htt-exon1(46Q) fibrillization by curcumin and EGCG and to determine the impact of these small molecules on aggregate morphology, AFM analysis was performed. This is particularly important to do with regard to EGCG, as it can compete with ThT binding to fibrils for some amyloid-forming proteins. ${ }^{29} \mathrm{Co}$-incubation experiments were performed with fresh preparations of htt-exon1(46Q) and with either curcumin or EGCG at a molar ratio of 5:1 (small molecule to protein). Control incubations of httexon1(46Q) without the small molecules were also analyzed. Htt-exon1(46Q) concentration was $20 \mu \mathrm{M}$. Representative AFM images of aliquots removed from solutions of htt-exon1(46Q) in the presence and absence of curcumin or EGCG after 1, 3, 5, and $8 \mathrm{~h}$ of incubation are shown in Fig 3.4. To quantify the effect of the small molecules on aggregation, AFM images from all incubations were analyzed by counting the number of fibrils and oligomers per $\mu \mathrm{m}^{2}$ (Fig. 3.4b) using automated scripts written in Matlab. For this analysis, oligomers were defined as any feature taller than $0.8 \mathrm{~nm}$ in height with an aspect ratio (longest distance across to shortest distance across) less than 3.0, indicating a globular structure. Fibrils were defined as aggregates taller than $0.8 \mathrm{~nm}$ in height with an aspect ratio greater than 3.0. These criteria were based on hand measured characteristics of representative examples of each aggregate type. The height threshold of $0.8 \mathrm{~nm}$ was chosen based on the background RMS roughness of mica that had been exposed to either curcumin or EGCG in the absence of htt (Fig. 3.5). 

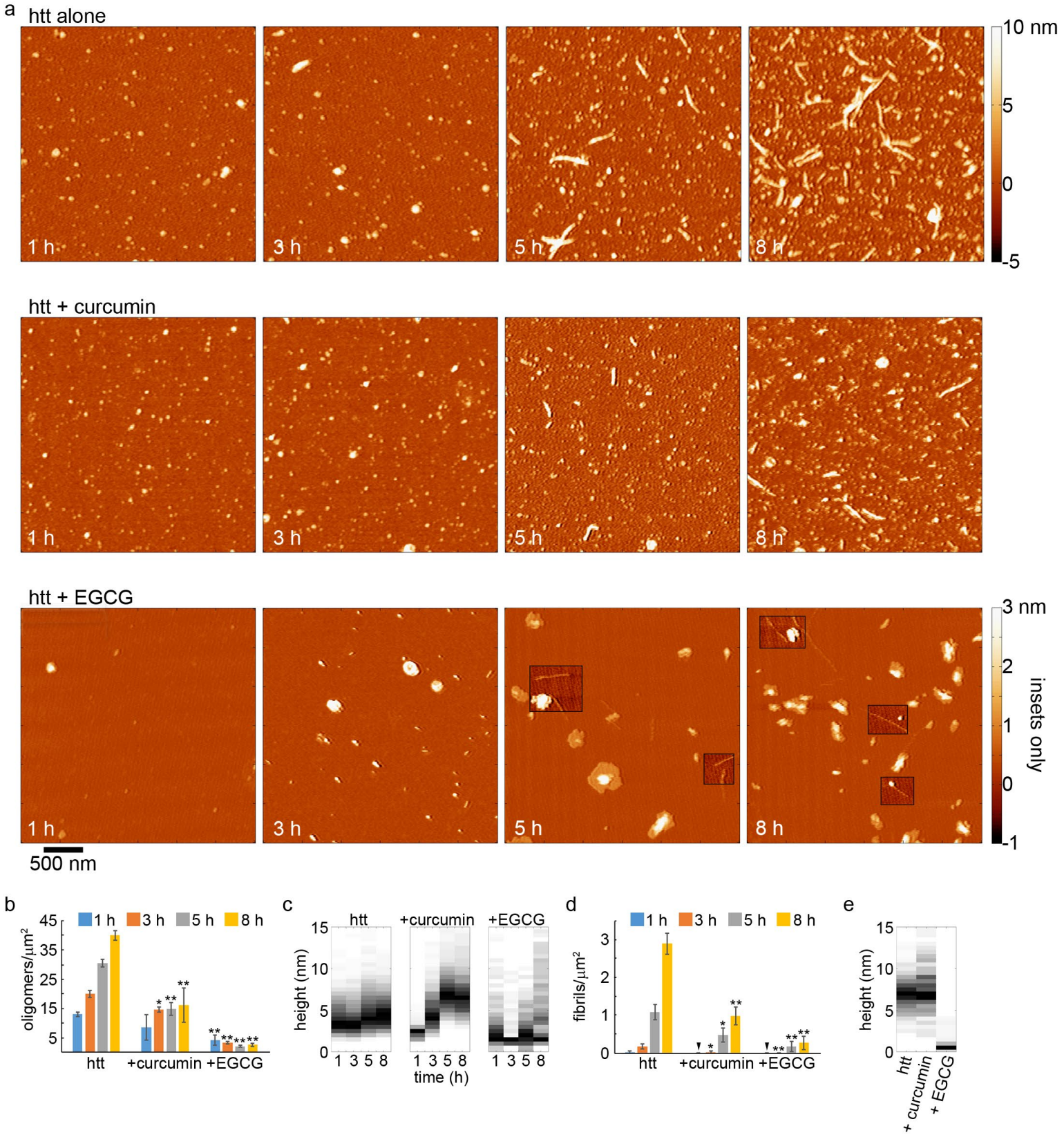

Figure 3.4 AFM analysis of the impact of curcumin or EGCG on htt-exon1(46Q) aggregation (ratio of 5:1 small molecule to htt). (a) Representative AFM images of $20 \mu \mathrm{M}$ htt-exon1(46Q) incubated alone, with curcumin, or with EGCG as a function of time. Colormap is the same for all images (see top color bar). The insets in the AFM images of htt incubated with EGCG uses a reduced color map (see bottom, labeled color bar) to make the shorter fibrils in these samples easier to see. (b) Image analysis of the number of oligomers per unit area as a function of time. (c) Height histograms of oligomers observed in AFM images as a as a function of time. (d) Image analysis of the number of fibrils per unit area as a function of time. (e) Height histograms of fibrils observed in AFM images as a function of time. For (b) and (d), error bars represent the standard deviation, and * represents a p value $<$ 0.05 , and ${ }^{* *}$ represents a $\mathrm{p}$ value $<0.01$ using a student's $\mathrm{T}$ test. 

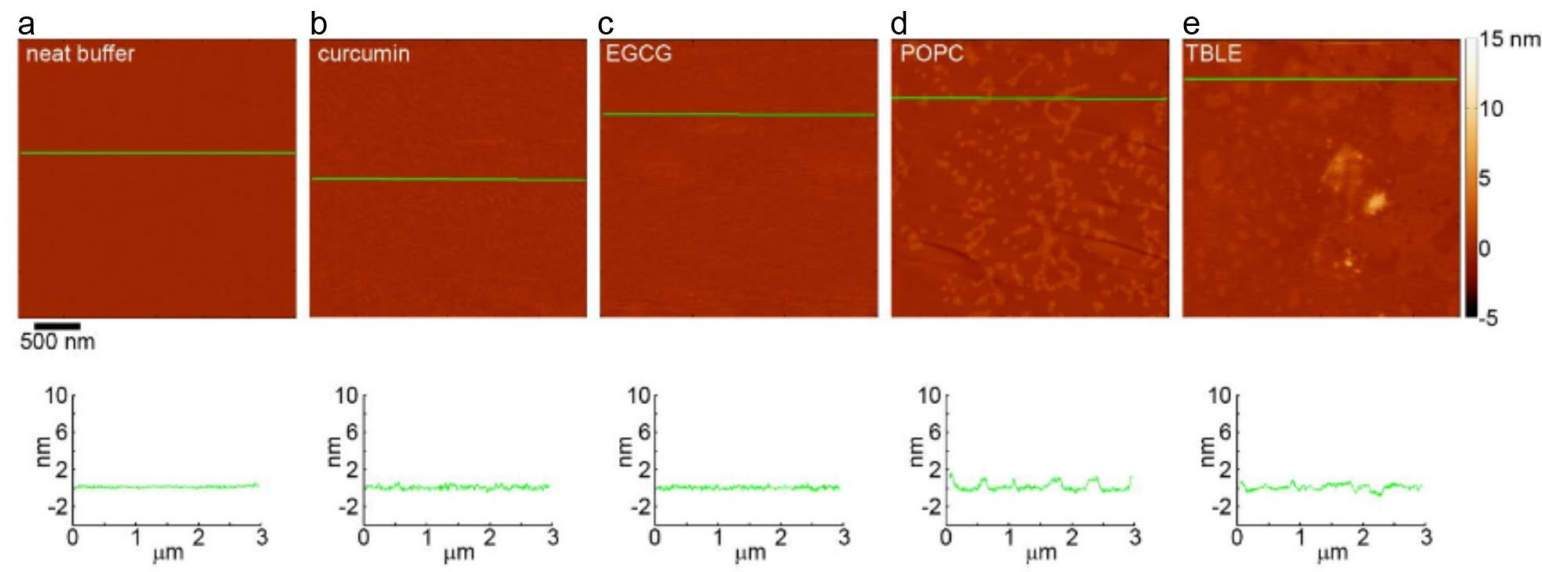

Figure 3.5 Small molecules and lipids result in AFM backgrounds with low RMS roughness. Representative AFM images of the backgrounds associated with (a) near buffer, (b) curcumin, (c) EGCG, (d) POPC lipid vesicles, and (e) TBLE vesicles. The line in each image corresponds to the height profile provided below each image.

Htt-exon1(46Q) incubated in the absence of small molecules exhibited extensive oligomer formation after $1 \mathrm{~h}$ of incubation with a steady increase in the number of oligomers with longer incubation times. After 1 and $3 \mathrm{~h}$ of incubation, oligomers were predominately 3-3.5 $\mathrm{nm}$ in height (Fig. 3.4a and b). Oligomers became slightly larger with time (a mode of 4-4.5 nm and 4.5-5 nm for 5 and $8 \mathrm{~h}$ time points respectively, Fig. 3.4c)). Fibrils appeared after $3 \mathrm{~h}$ of incubation in the htt-exon1(46Q) alone incubation, and the number of fibrils steadily increased with time (Fig 3.4a and d). While the number of fibrils increased, the average height along the fibril contours did not change with time (mode of 6.5-7.5 nm, Fig. 3.4e).

When curcumin was co-incubated with htt-exon1(46Q), the formation of aggregates was inhibited. Oligomers appeared in similar numbers as the control after $1 \mathrm{~h}$ of co-incubation with curcumin; however, the number of oligomers per unit area was significantly reduced from the $3 \mathrm{~h}$ time point for the duration of the experiment (Fig 3.4a and b). Fibrils were not detected until after $3 \mathrm{~h}$, and they were significantly reduced in number compared to control $(\mathrm{p}<0.05$, 
Fig. 3.4a and d). The fibril population continued to be significantly smaller in comparison to the control for the duration of the experiment ( $\mathrm{p}<0.05$ and $\mathrm{p}<0.01$ after 5 and $8 \mathrm{~h}$ respectively). While the number of oligomers and fibrils was reduced in the presence of curcumin, the size of the htt oligomers observed with curcumin was different compared to control experiments (Fig. 3.4c). At $1 \mathrm{~h}$, the mode height of oligomers formed in the presence of curcumin was $2.5 \mathrm{~nm}$. With longer incubation times, the oligomers became progressively larger with a mode height of 4.0-4.5 $\mathrm{nm}$ after $3 \mathrm{~h}$ and $6.5-7.0 \mathrm{~nm}$ after 5 and $8 \mathrm{~h}$ of co-incubation with curcumin. This was consistent with a previously reported tendency of conditions that slow htt fibrillization promoting larger oligomeric aggregates. ${ }^{15,63}$ Fibrils formed in the presence of curcumin were similar in morphology to control. The average height along the contour of the fibril did not change with time with an overall mode of 6.5-7.0 nm (Fig. 3.4e).

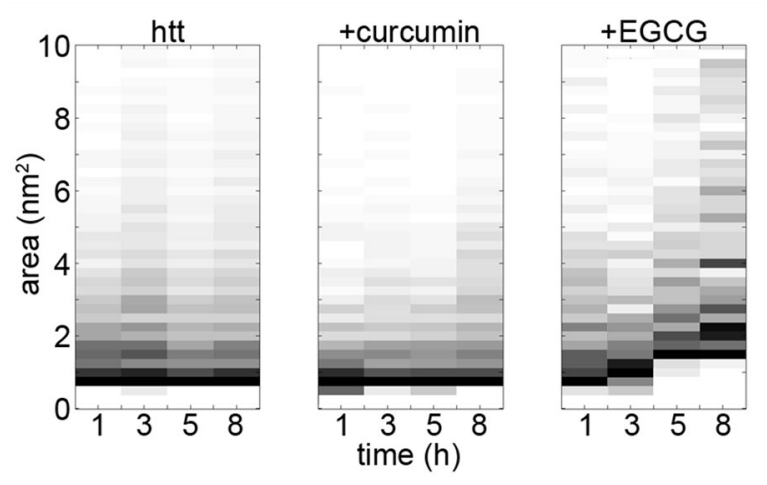
Figure 3.6 EGCG alters htt oligomer morphology. Histograms of the surface occupied by individual oligomers observed for htt-exon1(46Q) incubated alone, with curcumin, or EGCG.

When EGCG was co-incubated with htt-exon1(46Q), the formation of aggregates was robustly inhibited. Both oligomer and fibril formation were significantly decreased $(p<0.01)$ at all time points (Fig. 3.4a, b, and d). While the number of aggregates was reduced, the morphologies of the observed aggregates formed in the presence of EGCG were very different compared with those observed in control and + curcumin experiments (Fig. $3.4 \mathrm{c}$ and e). Aggregates distinguished as oligomers from our automated scripts were often large and 
conglomerate-looking in morphology. That is, these aggregates were granular in appearance, adopted a variety of shapes, and often had a flat ( $\sim 2-2.5 \mathrm{~nm}$ thick) region around its periphery. These aggregates were larger than the typical oligomers formed by htt, occupying a much larger area of the surface, and more heterogeneous in morphology (Fig. 3.6).The propensity to form these large aggregates of $\mathrm{htt}$ in the presence of EGCG has previously been reported. ${ }^{34}$ While a small population of fibrils were detected, the morphological characteristics of these fibrils were very different compared to control as they had a much smaller average height along their contours (mode of 1-1.5 nm) compared to fibrils formed by htt alone or with curcumin (Fig. 3.4e and 3.7). These thin, fibrillar species often (although not always) appeared to be growing from a larger, conglomerate aggregate (Fig. 3.7). This unique structure could represent a single protofilament that are incorporated into larger amyloid fibrils.

a
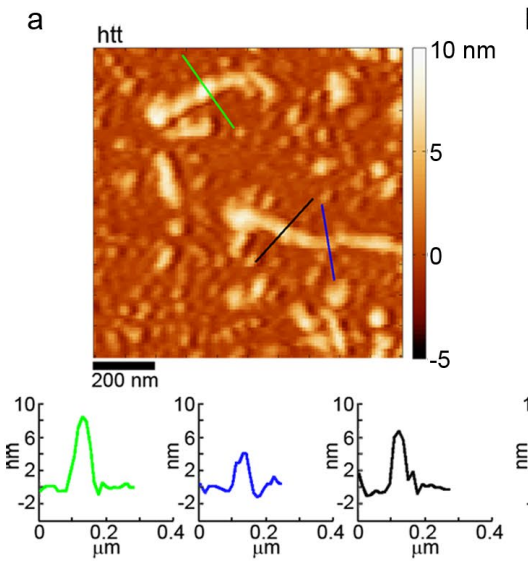

b
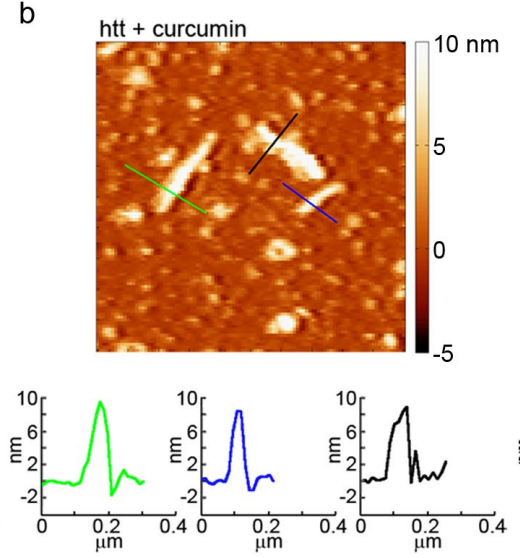

C
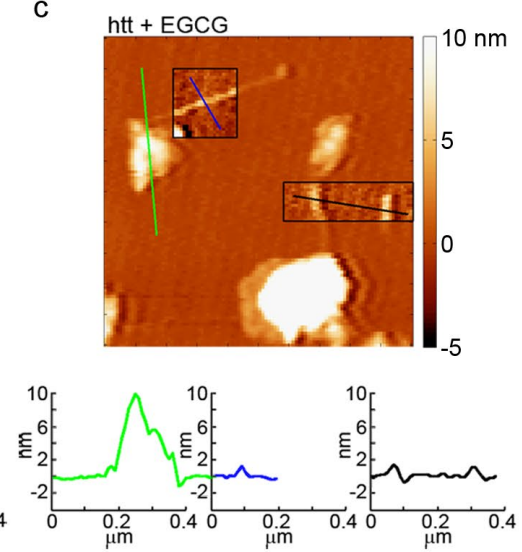

Figure 3.7 Htt aggregate morphology is altered by curcumin and EGCG. AFM images comparing the morphology of htt-exon1(46Q) fibrils formed in the (a) absence of small molecules, (b) with curcumin, or (c) with EGCG. In (c), the boxed insets correspond to the second color bar to better visualize the fibril structures associated with htt incubated with EGCG. The color lines in each image corresponds to the height profiles directly below each image. 


\subsubsection{Lipid membranes alter the ability of curcumin and EGCG to inhibit fibrillization}
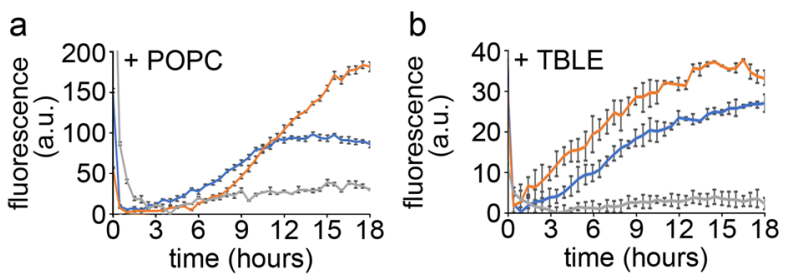

Figure 3.8 POPC and TBLE alter the ability of small molecules to inhibit htt-exon 1(46Q) fibrillization. ThT aggregation assays for htt-exon1(46Q) aggregated in the presence of (a) POPC or (b) TBLE lipid vesicles. Htt-
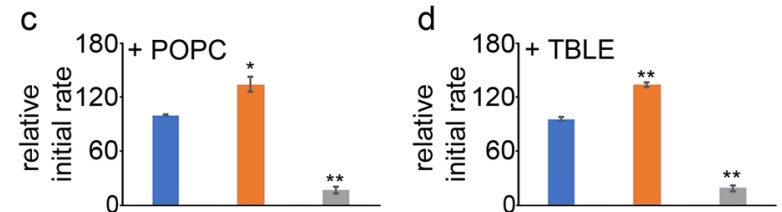
exon1(46Q) concentration was $20 \mu \mathrm{M}$ Curcumin and EGCG were added at a 5:1 molar ratio of small molecule to htt. The initial rate of aggregation in the presence of

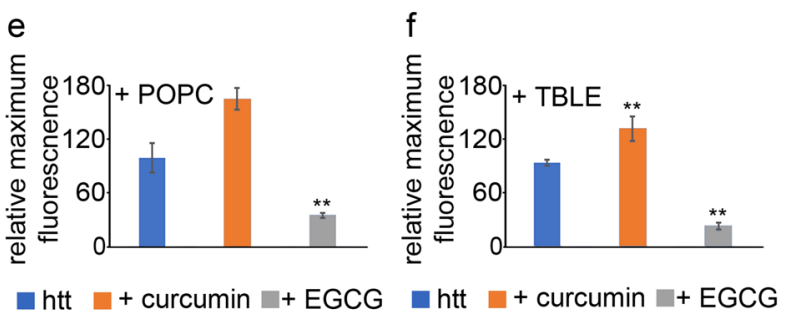

(c) POPC or (d) TBLE vesicles was made relative to the aggregation rate of htt with the lipid vesicles in the absence of the small molecules. Finally, the relative maximum fluorescence of each condition in the presence of (e) POPC or (f) TBLE lipid vesicles at the end of the $18 \mathrm{~h}$ kinetic run was compared. Analysis shown in (a) and (d) were determined as averages over all trials (shown in Fig. 3.3). Error bars are provided for every $6^{\text {th }}$ datapoint $(30 \mathrm{~min})$ and represent the standard error of the mean. * represents a $\mathrm{p}$ value $<0.05$ and $* *$ represents a $\mathrm{p}$ value $<0.01$.

To determine if the presence of lipid membranes impacts the ability of curcumin or

EGCG to alter htt aggregation, we used two lipid systems. POPC was selected as it was

previously shown to promote a distinct htt aggregation process. ${ }^{43}$ The other system was total brain lipid extract (TBLE) as it also alters htt aggregation ${ }^{40,64,65}$ and represents a more physiologically relevant mixture of lipids. Both lipid systems were introduced as vesicles, and the lipid to protein ratio was 20:1. Initially, the relative ability of curcumin and EGCG to inhibit htt fibril formation in the presence of POPC or TBLE vesicles was assessed by ThT aggregation assays (Fig. 3.8; all independent trials are shown in Fig. 3.3). As determined by DLS, the protocol used produced POPC vesicles with an average diameter $\sim 130-145 \mathrm{~nm}$ with a 
polydispersity index (PDI) of $\sim 0.34-0.4$ and TBLE vesicles with average diameter of $\sim 180-195$ $\mathrm{nm}$ with a PDI of $\sim 0.25-0.3$ (representative DLS distributions are presented in Fig. 3.9). The concentration of htt-exon 1(46Q) was $20 \mu \mathrm{M}$, and the small molecule to protein ratio was 5:1.

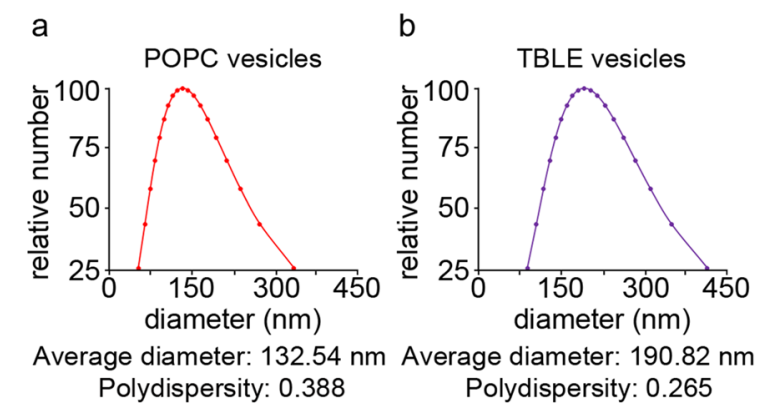

Figure 3.9 POPC and TBLE vesicles were comparable in size. Representative DLS analyses of (a) POPC and (b) TBLE vesicle size. Vesicle size distributions were calculated by assuming a lognormal distribution. Each plot is a combination of three runs.

The impact of exposing htt-exon1(46Q) to these two lipid systems (in the absence of small molecules) was varied. The addition of POPC accelerated htt aggregation compared to the absence of lipids; whereas, TBLE slowed htt aggregation (compare the fluorescence intensity of the htt alone experiments in Figs. 3.1 and 3.8). In the presence of POPC, curcumin was able to delay the onset of fibrillization, as a lag phase was clearly observed (Fig. 3.8a). However, fibril growth occurred rapidly once initiated, as the initial aggregation rate exceeded that of htt alone in the presence of POPC (35\% larger, Fig. 3.8c). The overall ability of curcumin to inhibit fibrillization was reduced in the presence of POPC, with the maximum ThT signal being $68 \%$ larger than control (Fig. 3.8e). In the presence of TBLE vesicles, htt aggregation proceeded faster without a discernable lag phase in the presence of curcumin compared to control $(35 \%$ increase in the initial aggregation rate, Fig. 3.8d), and the relative maximum ThT signal was increased by $40 \%$ compared to control (Fig. 3.8f). Htt-exon1(46Q) aggregation in the presence of POPC and TBLE vesicles was inhibited by EGCG (relative maximum ThT signals that 
were reduced to $41 \%$ and $22 \%$ compared to the control signal for incubations with POPC or TBLE respectively, Fig. 3.8a-f).

To further establish that POPC or TBLE vesicles altered the ability of curcumin and EGCG to inhibit htt-exon1(46Q) fibrillization, AFM analysis was performed. As before, coincubation experiments were performed with fresh preparations of htt-exon1(46Q) $(20 \mu \mathrm{M})$ and with either curcumin or EGCG at a molecular ratio of 5:1 (small molecule to protein), however, lipid vesicles of either POPC or TBLE were also added to the co-incubations at a 20:1 lipid to protein ratio. Control incubations of htt-exon1(46Q) with POPC and TBLE vesicles but without the small molecules were also performed (Figs. 3.10 and 3.11). Representative AFM images of aliquots removed from solutions of htt-exon1(46Q) in the presence and absence of curcumin or EGCG after 1,3,5, and $8 \mathrm{~h}$ of incubation in the presence of POPC or TBLE vesicles are shown in Fig. 3.10 and Fig. 3.11 respectively. To quantify the effect of the small molecules on aggregation in the presence of lipids, AFM images from all incubations were analyzed by counting the number of fibrils and oligomers per $\mu \mathrm{m}^{2}$ (Fig. 3.10 and 3.11) using automated scripts written in Matlab. As the addition of lipids to these incubations resulted in taller backgrounds once deposited on mica (Fig. 3.10), the height threshold used for image analysis was increased to $1.5 \mathrm{~nm}$. As a result, oligomers were defined as any feature taller than $1.5 \mathrm{~nm}$ in height with an aspect less than 3.0, indicating a globular structure. Fibrils were defined as aggregates taller than $1.5 \mathrm{~nm}$ in height with an aspect ratio greater than 3.0. 
$\mathrm{a}_{\mathrm{htt}}$ (POPC vesicles)
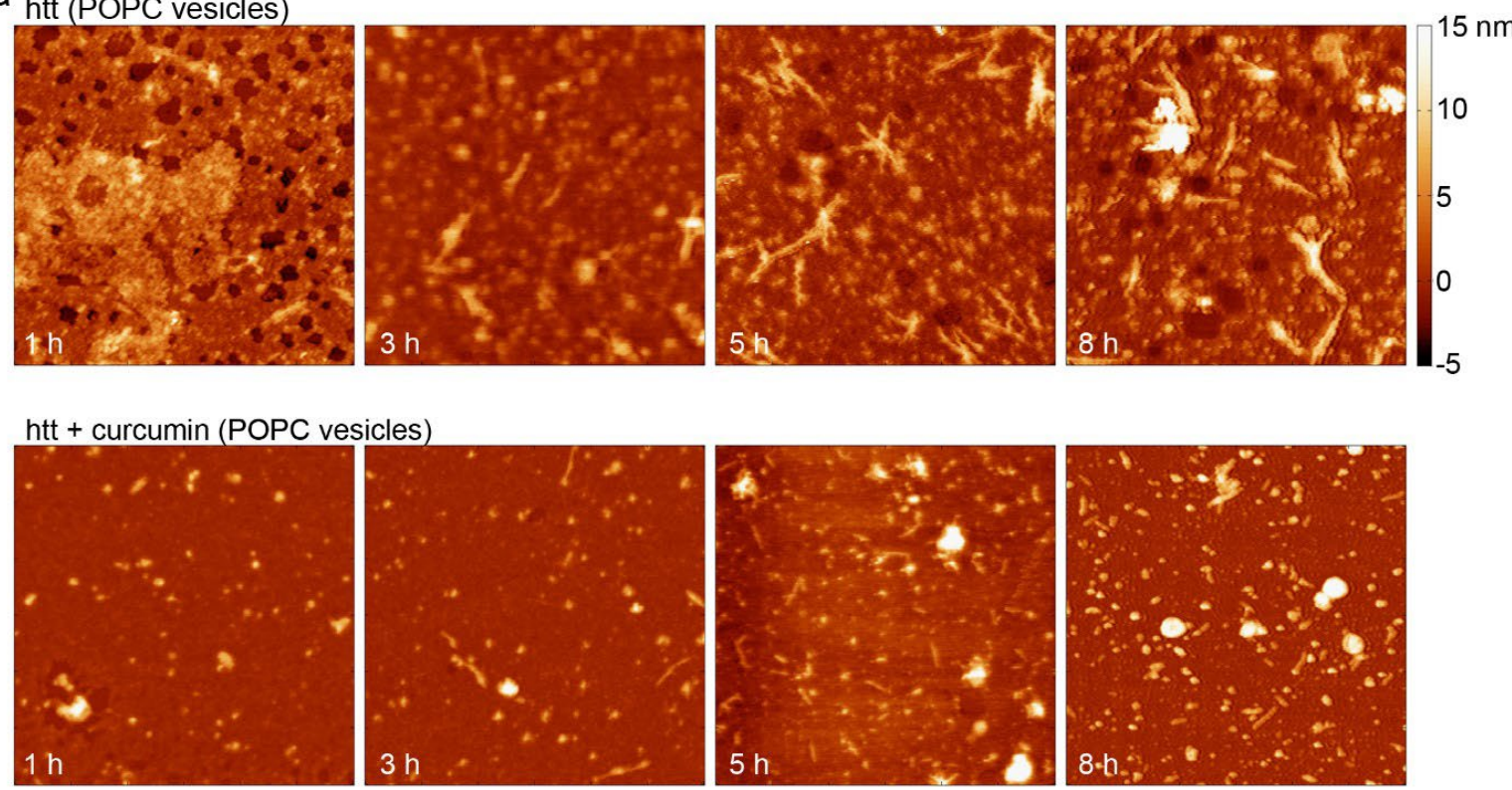

htt + EGCG (POPC vesicles)
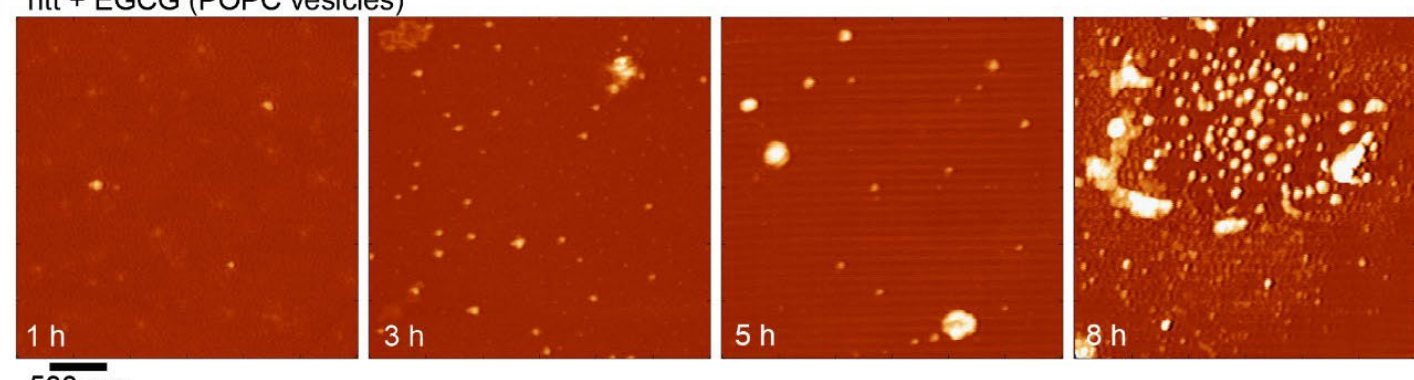

$5 \overline{00 \mathrm{~nm}}$

b

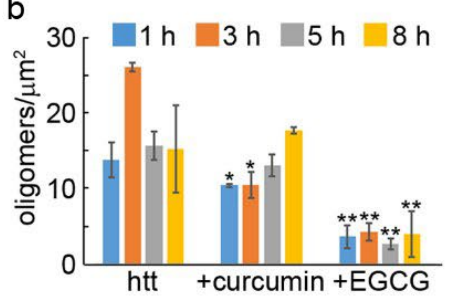

C

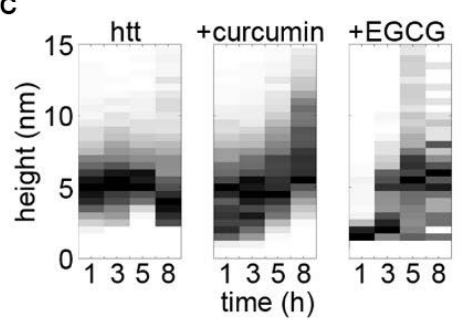

d

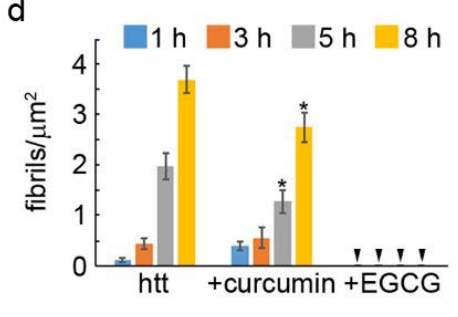

e

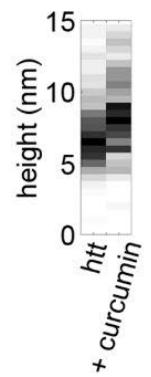

Figure 3.10 AFM analysis of the impact of curcumin or EGCG on htt-exon1(46Q) aggregation in the presence of POPC lipid vesicles. (a) Representative AFM images of $20 \mu \mathrm{M}$ htt-exon1(46Q) incubated with POPC (20:1 lipid to protein) with no small molecules, with curcumin, or with EGCG (ratio of 5:1 small molecule to htt) as a function of time. Color map is the same for all images. (b) Image analysis of the number of oligomers per unit area as a function of time. (c) Height histograms of oligomers observed in AFM images as a as a function of time. (d) Image analysis of the number of fibrils per unit area as a function of time. Arrows indicate that no fibrils were observed. (e) Height histograms of fibrils observed in AFM images as a function of time. For (b) and (d), error bars represent the standard deviation, and * represents a $p$ value $<0.05$, and $* *$ represents a $\mathrm{p}$ value $<0.01$ using a student's T test. 
htt (TBLE vesicles)
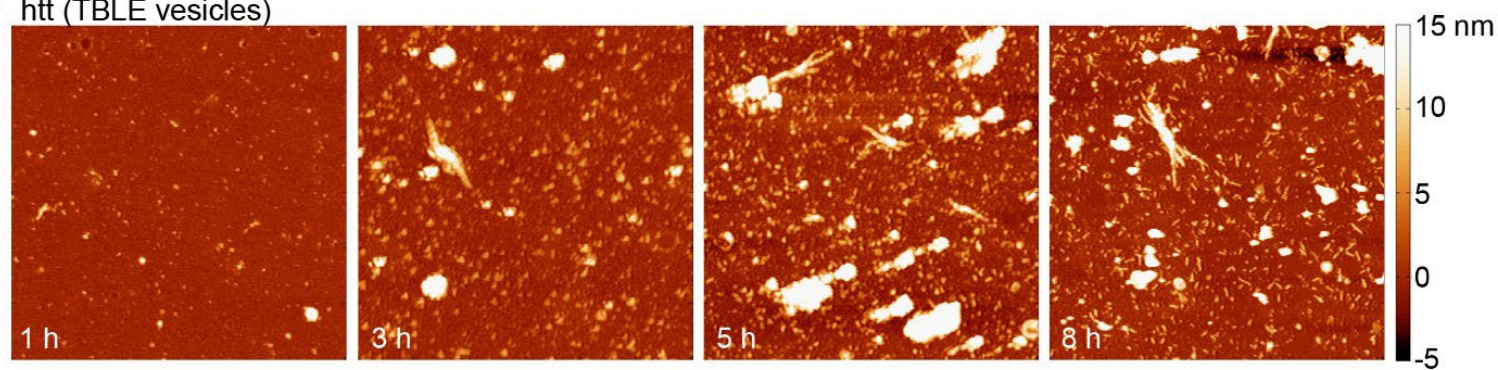

$\mathrm{htt}+$ curcumin (TBLE vesicles)
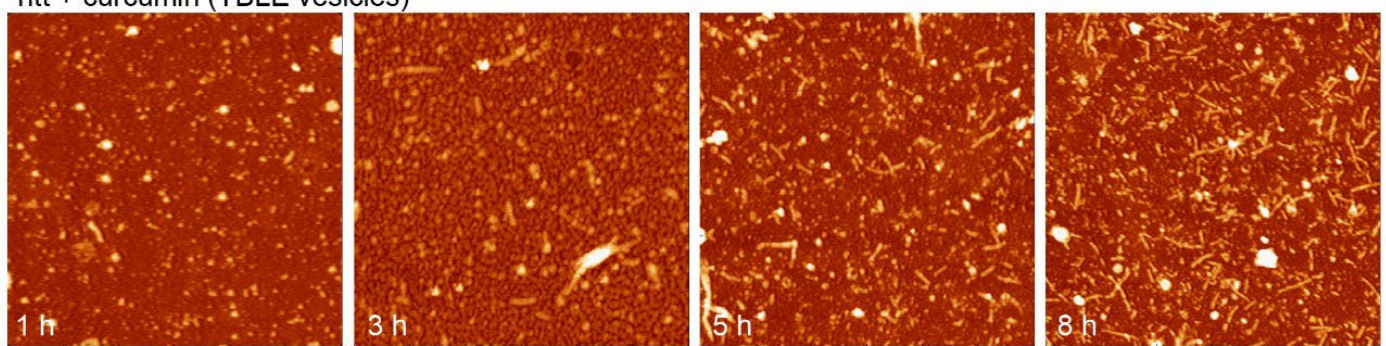

htt + EGCG (TBLE vesicles)
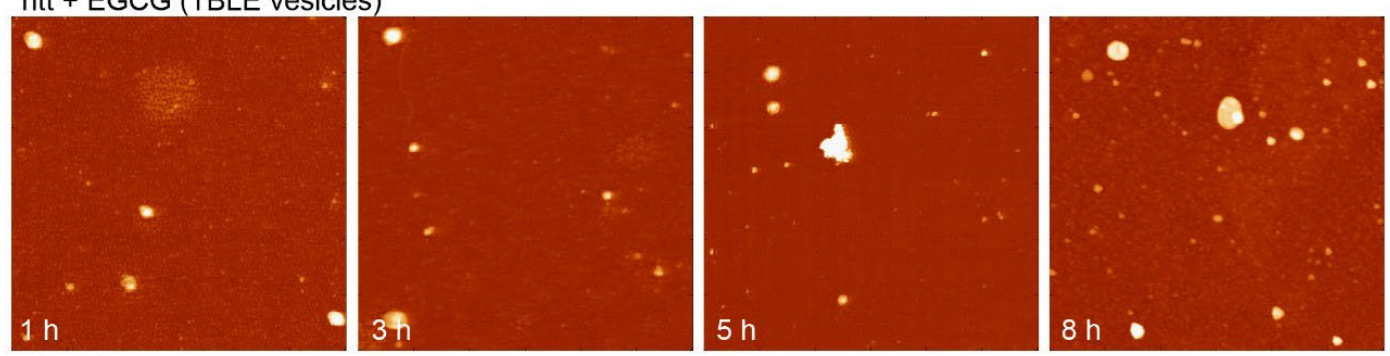

$$
500 \mathrm{~nm}
$$

b
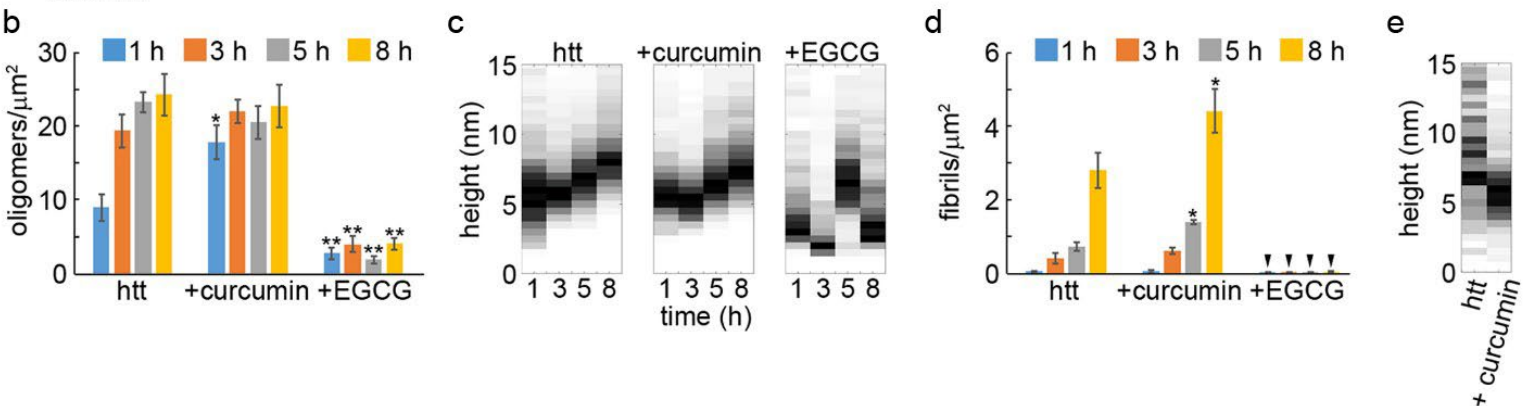

Figure 3.11 AFM analysis of the impact of curcumin or EGCG on htt-exon1(46Q) aggregation in the presence of TBLE vesicles. (A) Representative AFM images of $20 \mu \mathrm{M}$ htt-exon1(46Q) incubated with TBLE (20:1 lipid to protein) with no small molecules, with curcumin, or with EGCG (ratio of 5:1 small molecule to htt) as a function of time. Color map is the same for all images (B) Image analysis of the number of oligomers per unit area as a function of time. (C) Height histograms of oligomers observed in AFM images as a as a function of time. (D) Image analysis of the number of fibrils per unit area as a function of time. Arrows indicate that no fibrils were observed. (E) Height histograms of fibrils observed in AFM images as a function of time. As fibrils were not observed for htt-exon1(46Q) incubations with TBLE and EGCG, this condition is not presented in the fibril histograms. For (B) and (D), error bars represent the standard deviation, and * represents a $\mathrm{p}$ value $<0.05$, and $* *$ represents a $\mathrm{p}$ value $<$ 0.01 using a student's T test. 
Htt-exon1(46Q) incubated in the presence of POPC exhibited extensive oligomer formation after $1 \mathrm{~h}$ of incubation (Fig. 3.10). While the number of detectible oligomers increased after $3 \mathrm{~h}$, the oligomer population remained relatively stable during the time course of the experiment (Fig. 3.10b). After $1 \mathrm{~h}$ of incubation the mode height of oligomers was 4-5.5 nm (Fig. 3.10c). Oligomers were slightly larger at 3 and $5 \mathrm{~h}$ of incubation as the mode height was $5-6 \mathrm{~nm}$ at these time points. At $8 \mathrm{~h}$ of incubation, oligomers decreased in size with a mode height of $\sim 2.5-3.5 \mathrm{~nm}$. These oligomer species may contain lipid components; however, it is unlikely that they are comprised of lipids alone, as features observed from pure lipid incubations were all smaller than $1.5 \mathrm{~nm}$ in height and were not globular in appearance (Fig. 3.5). Fibrils appeared after $1 \mathrm{~h}$ of incubation in the htt-exon1(46Q) with POPC incubations, which was earlier than without any lipids (Fig 3.10a and d). The number of fibrils steadily increased with time, reaching a larger population compared with htt incubations without lipids, which is consistent with results from the ThT assays. While the number of fibrils increased, the average height along the fibril contours (mode of 6-7 nm) did not change with time and was comparable to fibrils formed in the absence of lipids (Fig. 3.4e and 3.10e).

When co-incubated with htt-exon1(46Q) in the presence of POPC, curcumin's ability to reduce aggregation was dampened (Fig. 3.10). While there was initially a significant reduction in oligomers, the number of oligomers formed at 5 and $8 \mathrm{~h}$ of co-incubation was not significantly different compared to incubations of htt-exon1(46Q) in the presence of POPC (Fig. 3.10b). There was, however, a larger distribution of oligomer sizes, yet the mode size of oligomers was comparable to those formed in the presence of POPC but the absence of curcumin at 1,3 , and $5 \mathrm{~h}$ (Fig. 3.10c). At $8 \mathrm{~h}$, the oligomer height distribution was shifted toward larger sizes. The promotion of larger oligomers by curcumin that was observed in the absence of lipid was much 
more subtle in the presence of POPC due to the larger distribution of oligomer sizes. Similar populations of fibrils were detected after 1 and $3 \mathrm{~h}$ from co-incubations with curcumin to control in the presence of POPC, and a small (but significant, $\mathrm{p}<0.05$ ) reduction in the fibril population was observed at 5 and $8 \mathrm{~h}$ (Fig. 3.10d). Fibrils formed in the presence of curcumin were similar in morphology to control (Figs. 3.10e and 3.12a). The average height along the contour of the fibril was larger compared with control fibrils, with a mode average height along the fibril contour of 8.5-9 nm; however, the distribution of these heights was also much broader, suggesting that lipids may be incorporated into the fibril structure.
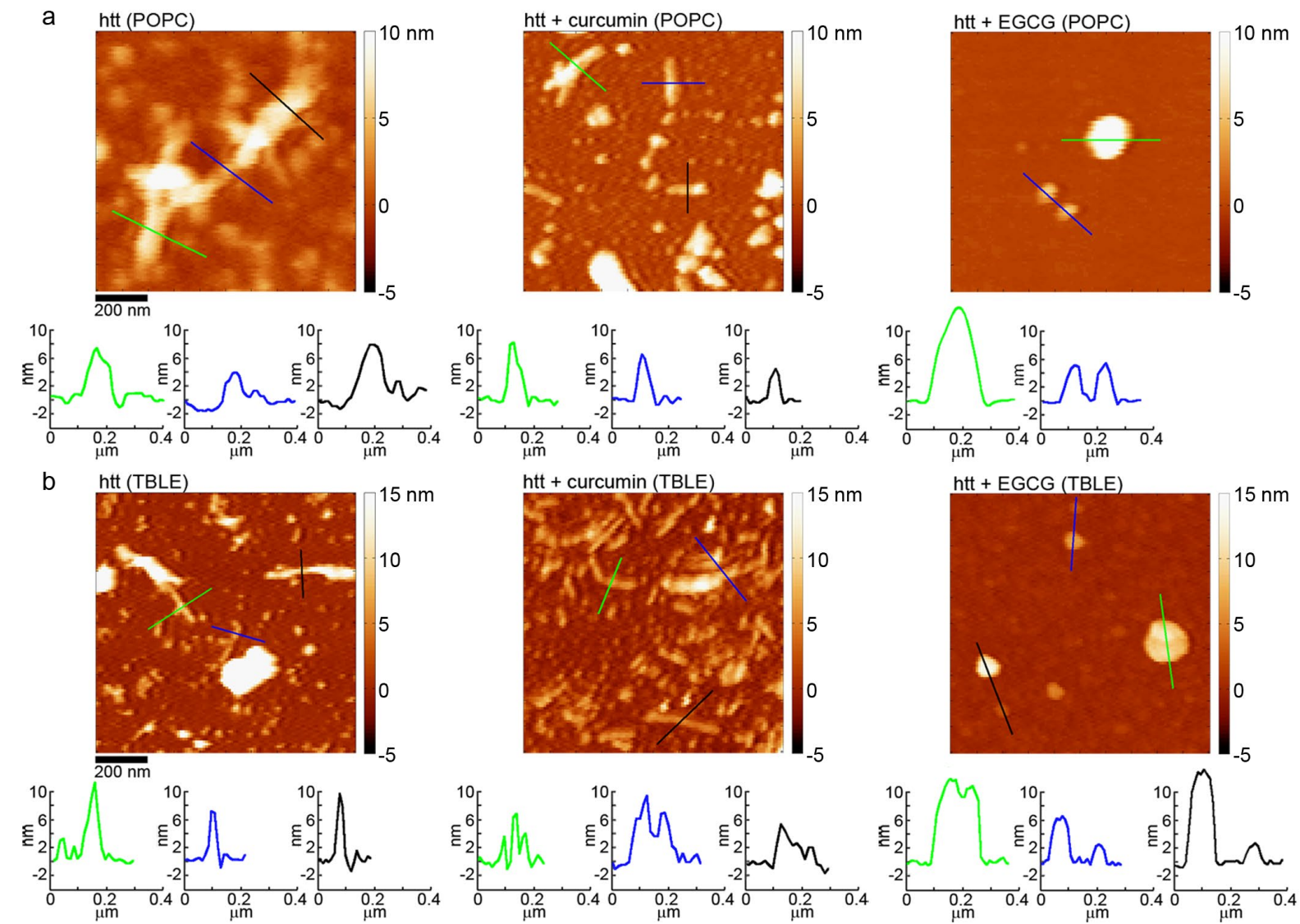

Figure 3.12 Curcumin and EGCG alter htt-exon1(46Q) aggregate morphology in the presence of lipid vesicles. AFM images comparing the morphology of htt-exon1(46Q) fibrils formed in the absence of small molecules, with curcumin, or with EGCG for incubations performed in the presence of (a) POPC or (b) TBLE vesicles. The color lines in each image corresponds to the height profiles directly below each image. 
When EGCG was co-incubated with htt-exon1(46Q) in the presence of POPC vesicles, EGCG was still an effective inhibitor of fibril formation (Fig. 3.10). The number of oligomers per unit area was significantly ( $\mathrm{p}<0.01$ at all time points) reduced compared to control (Fig 3.10b), and no fibrils were observed at any time point (Fig. 3.10d). While the number of oligomers was reduced, the morphologies of the observed oligomers formed in the presence of POPC vesicles and EGCG were very different compared with those in the control (Fig. 3.10c). There were a number of small oligomers $(\sim 2-3 \mathrm{~nm}$ in height), but there was also a large population of larger aggregates that could be considerably larger than $10 \mathrm{~nm}$ in height (Fig. 3.12a) and occupy large amounts of surface area (Fig. 3.13). The size of these aggregates was heterogeneous, and the ability of EGCG to promote large, globular htt aggregates was not diminished by the presence of POPC. However, unlike the aggregates observed when EGCG was incubated with htt alone, these species were more rounded and smooth in appearance.

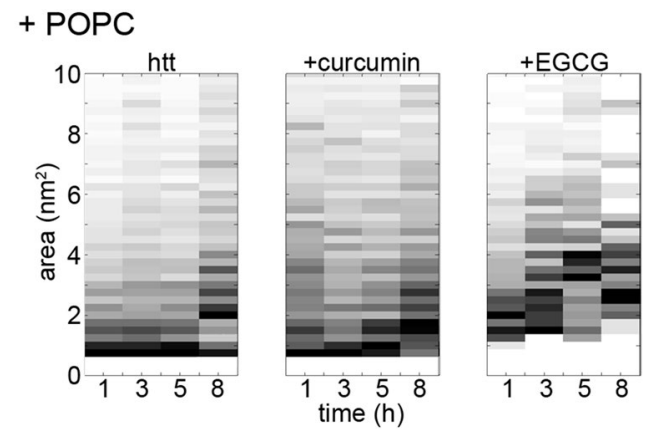

Figure 3.13 EGCG promotes large oligomeric aggregates of htt-exon1(46Q) in the presence of POPC. Histograms of the surface occupied by individual oligomers observed for htt-exon1(46Q) incubated alone, with curcumin, or EGCG in the presence of POPC lipid vesicles.

Htt-exon1(46Q) incubated in the presence of TBLE exhibited oligomer formation after 1 $\mathrm{h}$ of incubation with the population of oligomers increasing with time (Fig 3.11a and b). Unlike incubations in the absence of lipids or with POPC vesicles, the oligomers that formed in the presence of TBLE increased in size with time (going from a mode height of 5-6 $\mathrm{nm}$ at $1 \mathrm{~h}$ to a mode height of 7.5-8.5 nm after $8 \mathrm{~h}$, Fig. 3.11c). This increase in height is consistent 
with the observed trend that slowing fibril formation can promote large oligomeric species of htt to form. The number of fibrils per unit area (with TBLE) was smaller compared with incubations performed with POPC vesicles (Fig. 3.11c); however, fibril numbers were comparable with httexon1(46Q) incubations without lipids. Closer inspection of the AFM images suggests that the fibrils formed in the presence of TBLE were shorter than those grown in the absence of lipids, which could result in the reduced ThT signal. That is, the longer fibrils formed in the absence of TBLE would have more surface area (due to more monomers per fibril) to bind ThT, producing a larger signal. Despite this apparent difference in fibril length, the average height along the fibril contours (mode of 6.5-7 nm) of fibrils formed in the presence of TBLE did not change with time and was comparable to fibrils formed in the absence of lipids and POPC (Fig 3.11e and 3.12b).

When co-incubated with htt-exon1(46Q) in the presence of TBLE, curcumin no longer reduced aggregation (Fig 3.11). After $1 \mathrm{~h}$, a significant increase in the htt oligomer population was observed for co-incubations with curcumin (Fig. 3.11b). The large number of oligomers per unit area was stable at later time-points, and the numbers were comparable to control. The height distributions of oligomers formed from co-incubations of htt with curcumin in the presence of TBLE was very comparable to control at all time points (Fig. 3.11c). Consistent with the ThT analysis, the number of fibrils formed from co-incubations of curcumin in the presence of TBLE was significantly larger compared with control at 5 and $8 \mathrm{~h}$ (Fig. 3.11d). The average height along the contour of the fibril was larger for control fibrils, with a mode average height along the fibril contour of 8.5-9 nm (Fig. 3.11e); however, the distribution of these heights was also much broader. The mode average height along the contour was $5-6.5 \mathrm{~nm}$ for fibrils formed from coincubations with curcumin in the presence of TBLE. The thickness along these fibrils formed in the presence of TBLE (in the absence and presence of curcumin) were quite varied (Fig. 3.12b). 


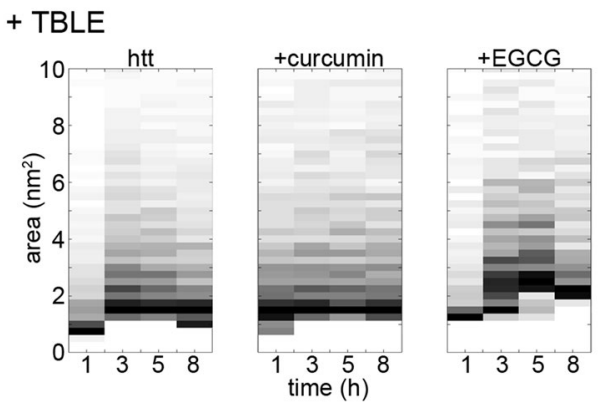

Figure 3.14 EGCG promotes large oligomeric aggregates of htt-exon1(46Q) in the presence of TBLE. Histograms of the surface occupied by individual oligomers observed for htt-exon1(46Q) incubated alone, with curcumin, or EGCG in the presence of TBLE lipid vesicles.

When EGCG was co-incubated with htt-exon1(46Q) in the presence of TBLE, the formation of aggregates was robustly inhibited, as the number of oligomers per unit area was significantly reduced compared to control (Fig 3.11). Oligomer and fibril formation was significantly $(\mathrm{p}<0.01)$ decreased at all time points (Fig. 3.11b and d). While the number of aggregates was reduced, the morphologies of the observed aggregates formed in the presence of EGCG were very different compared with those observed in control and + curcumin experiments (Fig. 3.11c and 3.12b). Similar to incubations in the presence of POPC, aggregates distinguished as oligomers from our automated scripts were often large, conglomerate-looking in morphology when incubated with TBLE vesicles. These aggregates were larger than the typical oligomers formed by htt, occupying a much larger area of the surface, and more heterogeneous (Fig. 3.14). Again, the lipid surfaces did not impede the ability of EGCG to prevent htt fibril formation and promote large globular aggregates. These large, non-fibrillar aggregates tended to be smooth and rounded similar to those observed with EGCG and htt in the presence of POPC. At the $8 \mathrm{~h}$ time point, some of these larger aggregates had a flat, peripheral feature. However, this feature was distinct from the peripheral featured observed for EGCG and htt incubation in the absence of lipids as these flat regions were between $5-10 \mathrm{~nm}$ in height, suggesting that these might be lipid bilayer stacks. 

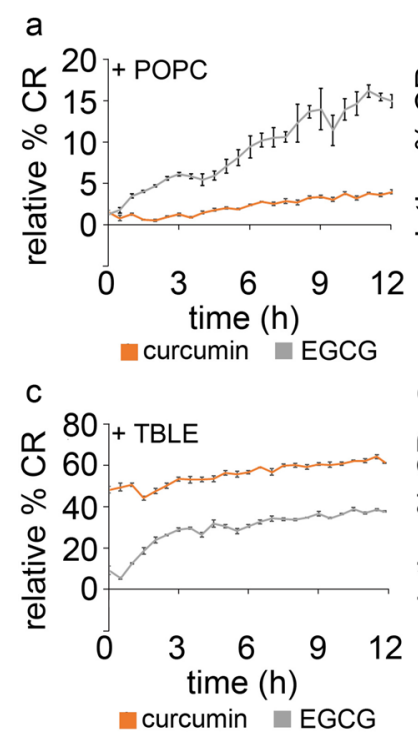

b

20 ]+ POPC

U 15

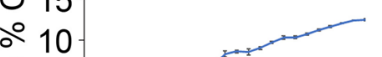

5

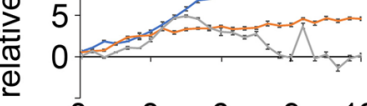

$\begin{array}{llll}3 & 6 & 9 & 12\end{array}$ ntt $\quad+$ curcumin + EGCG

d

${ }^{80}+0^{80}+$ TBLE

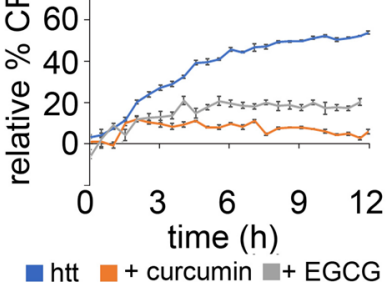

Figure 3.15 Curcumin and EGCG alter htt lipid binding. Lipid binding assays for (a) PDA/POPC vesicles exposed to either curcumin or EGCG, (b) PDA/POPC vesicles exposed to httexon1(46Q) with either curcumin or EGCG, (c) PDA/TBLE vesicles exposed to either curcumin or EGCG, and (d) PDA/TBLE vesicles exposed to httexon1(46Q) with either curcumin or EGCG. Error bars are provided for every $6^{\text {th }}$ datapoint and represent the standard error of the mean.
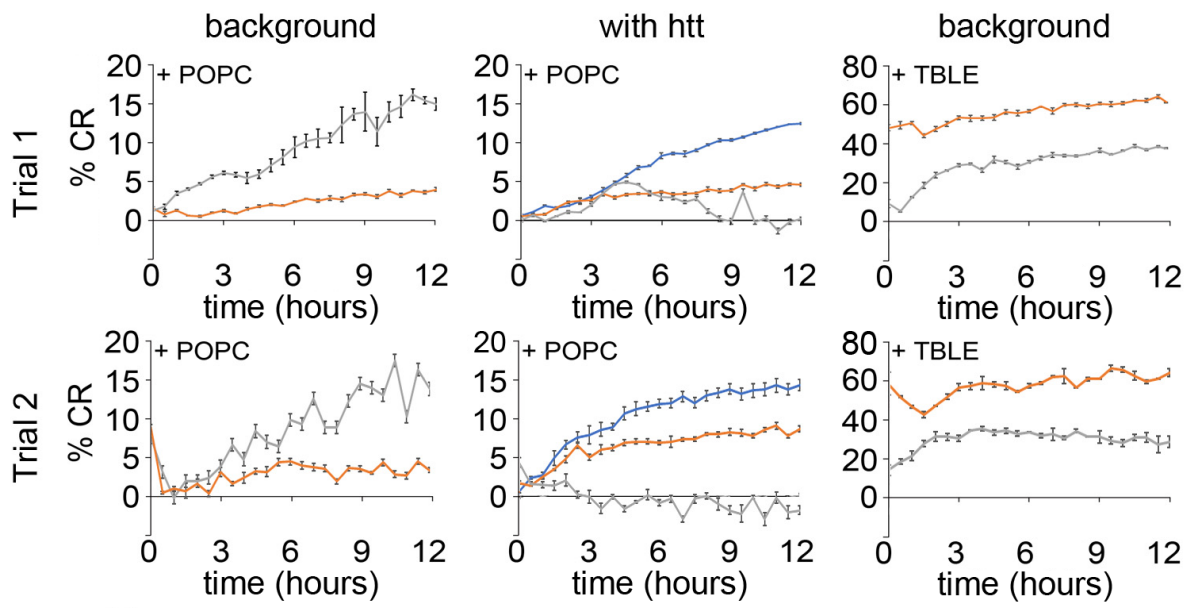

time (hours)
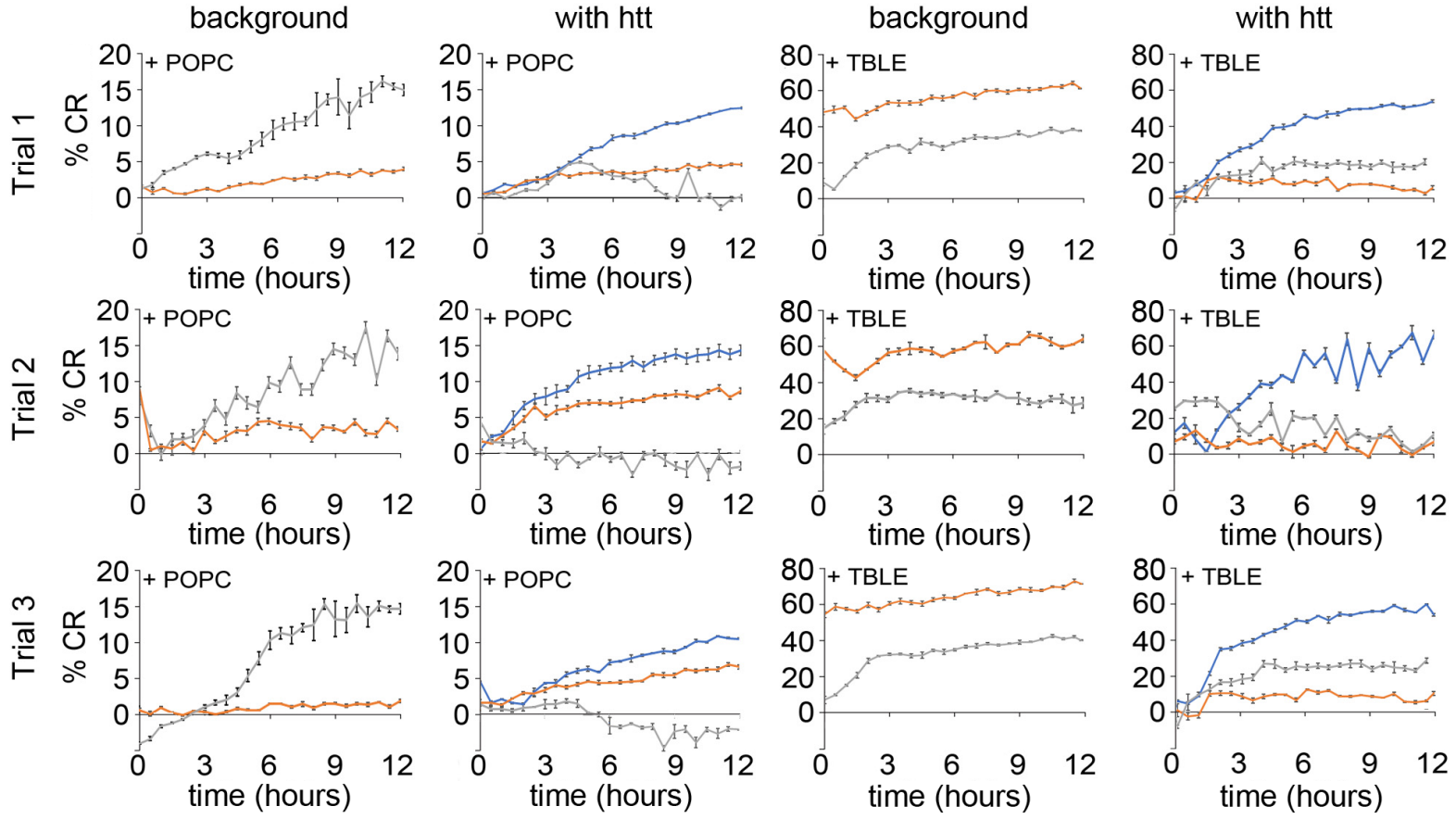

htt $\quad+5: 1$ curcumin $\quad+5: 1$ EGCG

Figure 3.16 Individual trials of the PDA lipid binding assays are consistent.

Background plots of the lipid/PDA vesicles in the presence of $100 \mu \mathrm{M}$ curcumin (orange) or EGCG (gray). Assays performed with htt have subtracted out the appropriate small molecule background, so the signal shown is that associated specifically with the htt in the presence of curcumin (orange) or EGCG (gray). 
As the presence of lipid vesicles altered the ability of curcumin and EGCG to inhibit fibril formation, we next performed a performed a series of colorimetric membrane binding assays using lipid/polydiacetylene (PDA) vesicles (Fig. 3.15). These vesicles were made with either POPC or TBLE as the lipid component. Lipid/PDA vesicles have varied colorimetric responses (CRs) when exposed to proteins or small molecules depending on the extent of interaction. ${ }^{52,53}$ The $\mathrm{CR}$ in the lipid/PDA vesicles is related to transitions of the PDA polymer backbone structure, which is affected by changes in tension associated with exogenous molecules interacting with and/or inserting into the vesicle. ${ }^{53}$ By measuring absorbance of both the blue $(640 \mathrm{~nm})$ and red $(500 \mathrm{~nm})$ wavelengths of lipid/PDA vesicles upon exposure to httexon1(46Q), the \% CR was obtained, which directly corresponds to the protein/lipid interaction. Importantly, the $\% \mathrm{CR}$ response takes into account background experiments performed with the small molecules, so the response associated with curcumin or EGCG in the absence of htt is easily removed to obtain the \% CR associated specifically with the htt. These background experiments with just curcumin or EGCG can be compared with neat buffer to determine the extent to which each small molecule interacts with the lipids as well (all independent PDA assays are shown in Fig. 3.16). With POPC/PDA vesicles, curcumin by itself invoked a minimal $\% \mathrm{CR}$, demonstrating that curcumin has little interaction with POPC (Fig. 3.15a). However, the $\% \mathrm{CR}$ of curcumin alone was extensive with TBLE/PDA vesicles, starting as high as $45 \%$ and reaching over $60 \% \mathrm{CR}$ within hours, suggesting that curcumin strongly binds to TBLE (Fig. 3.15c). EGCG, which is known to bind lipids, ${ }^{57}$ interacted with both POPC and TBLE, with a slightly larger interaction with TBLE (Fig. 3.15a and c). Htt-exon1(46Q) by itself had a stronger $(\sim 3 \times)$ interaction with TBLE/PDA vesicles in comparison with POPC/PDA vesicles. The 
addition of curcumin or EGCG reduced the interaction of htt-exon1(46Q) with both POPC/PDA and TBLE/PDA vesicles (Fig. 3.15b and d).

\subsection{Discussion}

Amyloid formation is highly dependent on environmental factors, complicating efforts to design effective small molecules that inhibit disease-related protein aggregation. A major environmental factor that influences the aggregation of numerous amyloid-forming proteins is the presence of cellular and subcellular membranes comprised predominately of lipids. Here, we investigated how the presence of lipid vesicles altered the ability of two known amyloid inhibitors, curcumin and EGCG, to prevent htt fibrillization. First, we verified that both curcumin and EGCG inhibited fibril formation of htt-exon1(46Q) in the absence of lipid membranes. In particular, EGCG promoted unique htt aggregate species compared to controls, i.e. large, globular aggregates and thin protofilaments. Next, we determined that POPC enhances htt fibril formation; whereas, TBLE reduced htt fibril formation. In the presence of lipid vesicles of either POPC or TBLE, curcumin became ineffective in inhibiting htt fibrillization and even promoted fibril formation in the presence of TBLE. In contrast, EGCG remained effective in preventing htt fibrillization in the presence of lipid vesicles; however, some small oligomers and large conglomerate aggregates still formed. Collectively, these results underscore the complexity of htt aggregation and targeting fibril formation with small molecules.

Surfaces, especially lipid membranes, are known to heavily influence amyloid formation, even promoting unique aggregation pathways. ${ }^{50}$ Results presented here that the ability of curcumin and EGCG to alter htt aggregation is modified by the presence of lipid vesicles further support this notion. For htt, taking into account the influence of lipid membranes in the 
aggregation process of htt may play a key role in targeting the aggregation process as a therapeutic strategy, as htt and its aggregate forms are often intimately linked to a variety of membranes. ${ }^{52}$ For example, htt localizes to specific subcellular compartments as a result of its normal function via lipid interactions, ${ }^{66}$ participates in the transport of lipid vesicles (endocytic, synaptic or lysosomal) ${ }^{53,67-69}$ is associated with acidic phospholipids, ${ }^{51}$ and localizes to brain membrane fractions. ${ }^{70} \mathrm{Htt}$ also localizes to the ER, ${ }^{66,71-74}$ mitochondria, ${ }^{75-80}$ and plasma membrane. ${ }^{51}$ Literature reports have suggested both enhanced ${ }^{43}$ and inhibited $\mathrm{d}^{40,63,64,81} \mathrm{htt}$ fibrillization in the presence of membranes. This discrepancy appears to be due to properties of the particular lipid systems used in these studies and can be modified by changes in membrane composition. $^{41,42}$

Curcumin's inability to effectively inhibit fibril formation in the presence of lipids may be due to a variety of reasons. In the assays performed here, it is important to note that htt aggregation is likely occurring both on the membrane surface and in solution, creating a complex system. Curcumin initially appeared to delay the onset of htt aggregation in the presence of POPC vesicles, but this ability dissipated with time. Htt aggregation on POPC/POPS bilayers proceeds via a unique mechanism compared to aggregation in solution. ${ }^{43}$ A plausible scenario is that curcumin is more effective against the pathway associated with aggregation in solution than that occurring on the POPC membrane. However, curcumin did reduce the overall interaction of htt with the POPC bilayer, and curcumin itself did not significantly interact with the lipid surface. Perhaps more interestingly, curcumin increased fibril formation in the presence of TBLE vesicles. Unlike POPC, TBLE appears to reduce htt fibril formation. ${ }^{40,63,82}$ Based on the PDA assay, curcumin by itself strongly interacted with the vesicle surface, suggesting that the curcumin was sequestered within the vesicle. In addition, the interaction of htt with the TBLE 
vesicles was greatly inhibited by the presence of curcumin. Collectively, this suggests a scenario in which curcumin is quickly taken up by the TBLE vesicles, altering the membrane. This alteration reduces the amount of htt bound and aggregating on the TBLE vesicle surface, which is a slower fibril-forming pathway. As a result, there is a larger amount of htt aggregating in solution in the presence of curcumin compared with controls of htt with just the TBLE vesicles. As the curcumin that would inhibit fibrillization in solution has been sequestered by the TBLE vesicles, the portion of htt in solution is free to aggregate. As a result, fibrillization increased in the presence of curcumin and TBLE vesicles. While investigated for its interaction with $A \beta$, it has also been suggested that curcumin binds to later stage aggregate species like protofibrils. ${ }^{83,84}$ If curcumin also targets later stages of the aggregation process of htt, this too could contribute to its differing abilities to modify htt fibrillization in the presence and absence of lipids, especially if the aggregation process in the presence of lipids is altered.

EGCG remained effective in preventing htt fibril formation in the presence of lipid vesicles. The effectiveness of EGCG as an amyloid inhibitor in the presence of lipids appears to be protein specific, as EGCG was unable to prevent IAPP fibrillization on a lipid membrane ${ }^{85}$ but was more effective in preventing $A \beta$ aggregation in the presence of vesicles ${ }^{57}$ EGCG is known to be incorporated into lipid vesicles, ${ }^{57}$ and we did observe that EGCG by itself invoked a colorimetric response in our PDA assays, verifying that it directly interacted with both POPC and TBLE. EGCG appears to target early events in htt aggregation, ${ }^{34}$ and this also appears to be the case for EGCG's interaction A $\beta$ and $\alpha$-synuclein aggregation. ${ }^{27}$ This ability to intervene in the htt aggregation process at an early stage could be why EGCG remained an effective inhibitor in the presence of lipids. Even though EGCG remained effective in the presence of both lipid 
systems and promoted larger, nonfibrillar aggregates, the morphology of these large aggregates

was distinct, suggesting that lipid vesicles did influence how EGCG interacts with htt.

\subsection{References}

1. Cline, E. N., Bicca, M. A., Viola, K. L. \& Klein, W. L. The Amyloid- $\beta$ Oligomer Hypothesis: Beginning of the Third Decade. J. Alzheimers. Dis. 64, S567-S610.

2. Martial, B., Lefèvre, T. \& Auger, M. Understanding amyloid fibril formation using protein fragments: structural investigations via vibrational spectroscopy and solid-state NMR. Biophys. Rev. 10, 1133-1149 (2018).

3. Adegbuyiro, A., Sedighi, F., Pilkington, A. W., Groover, S. \& Legleiter, J. Proteins Containing Expanded Polyglutamine Tracts and Neurodegenerative Disease. Biochemistry 56, 1199-1217 (2017).

4. Lotz, G. P. \& Legleiter, J. The role of amyloidogenic protein oligomerization in neurodegenerative disease. J. Mol. Med. 91, 653-664 (2013).

5. Sengupta, U., Nilson, A. N. \& Kayed, R. The Role of Amyloid- $\beta$ Oligomers in Toxicity, Propagation, and Immunotherapy. EBioMedicine. 6, 42-49 (2016).

6. Campioni, S. et al. A causative link between the structure of aberrant protein oligomers and their toxicity. Nat. Chem. Biol. 6, 140-147 (2010).

7. Fraser, P. E., Nguyen, J. T., Surewicz, W. K. \& Kirschner, D. A. pH-dependent structural transitions of Alzheimer amyloid peptides. Biophys. J. 60, 1190-1201 (1991).

8. Petkova, A. T. et al. Solid state NMR reveals a pH-dependent antiparallel beta-sheet registry in fibrils formed by a beta-amyloid peptide. J. Mol. Biol. 335, 247-260 (2004).

9. Faller, P., Hureau, C. \& Berthoumieu, O. Role of Metal Ions in the Self-assembly of the Alzheimer's Amyloid- $\beta$ Peptide. Inorg. Chem. 52, 12193-12206 (2013).

10. Kodali, R., Williams, A. D., Chemuru, S. \& Wetzel, R. A $\beta(1-40)$ forms five distinct amyloid structures whose $\beta$-sheet contents and fibril stabilities are correlated. J. Mol. Biol. 401, 503-517 (2010).

11. Penney, J. B., Vonsattel, J. P., MacDonald, M. E., Gusella, J. F. \& Myers, R. H. CAG repeat number governs the development rate of pathology in Huntington's disease. Ann. Neurol. 41, 689-692 (1997).

12. Snell, R. G. et al. Relationship between trinucleotide repeat expansion and phenotypic variation in Huntington's disease. Nat. Genet. 4, 393-397 (1993). 
13. Tobin, A. J. \& Signer, E. R. Huntington's disease: the challenge for cell biologists. Trends Cell Biol. 10, 531-536 (2000).

14. Landrum, E. \& Wetzel, R. Biophysical Underpinnings of the Repeat Length Dependence of Polyglutamine Amyloid Formation. J. Biol. Chem. 289, 10254-10260 (2014).

15. Legleiter, J. et al. Mutant Huntingtin Fragments Form Oligomers in a Polyglutamine Lengthdependent Manner in Vitro and in Vivo. J. Biol. Chem. 285, 14777-14790 (2010).

16. Poirier, M. A. et al. Huntingtin spheroids and protofibrils as precursors in polyglutamine fibrilization. J. Biol. Chem. 277, 41032-41037 (2002).

17. Ruggeri, F. S. et al. Nanoscale studies link amyloid maturity with polyglutamine diseases onset. Sci. Rep. 6, 31155 (2016).

18. Hands, S. L. \& Wyttenbach, A. Neurotoxic protein oligomerisation associated with polyglutamine diseases. Acta. Neuropathol. 120, 419-437 (2010).

19. Morozova, O. A., Gupta, S. \& Colby, D. W. Prefibrillar huntingtin oligomers isolated from HD brain potently seed amyloid formation. FEBS Lett. 589, 1897-1903 (2015).

20. Olshina, M. A. et al. Tracking mutant huntingtin aggregation kinetics in cells reveals three major populations that include an invariant oligomer pool. J. Biol. Chem. 285, 21807-21816 (2010).

21. Kim, S. A. et al. Sedimentation Velocity Analysis with Fluorescence Detection of Mutant Huntingtin Exon 1 Aggregation in Drosophila melanogaster and Caenorhabditis elegans. Biochemistry 56, 4676-4688 (2017).

22. Ramdzan, Y. M. et al. Conformation sensors that distinguish monomeric proteins from oligomers in live cells. Chem. Biol. 17, 371-379 (2010).

23. Sathasivam, K. et al. Identical oligomeric and fibrillar structures captured from the brains of R6/2 and knock-in mouse models of Huntington's disease. Hum. Mol. Genet. 19, 65-78 (2010).

24. Kim, Y. E. et al. Soluble Oligomers of PolyQ-Expanded Huntingtin Target a Multiplicity of Key Cellular Factors. Mol. Cell 63, 951-964 (2016).

25. Nie, Q., Du, X. \& Geng, M. Small molecule inhibitors of amyloid $\beta$ peptide aggregation as a potential therapeutic strategy for Alzheimer's disease. Acta. Pharmacol. Sin. 32, 545-551 (2011).

26. Doig, A. J. \& Derreumaux, P. Inhibition of protein aggregation and amyloid formation by small molecules. Curr. Opin. Struct. Biol. 30, 50-56 (2015).

27. Bieschke, J. et al. EGCG remodels mature alpha-synuclein and amyloid-beta fibrils and reduces cellular toxicity. Proc. Natl. Acad. Sci. U.S.A. 107, 7710-7715 (2010). 
28. Rezai-Zadeh, K. et al. Green tea epigallocatechin-3-gallate (EGCG) reduces beta-amyloid mediated cognitive impairment and modulates tau pathology in Alzheimer transgenic mice. Brain Res. 1214, 177-187 (2008).

29. Palhano, F. L., Lee, J., Grimster, N. P. \& Kelly, J. W. Toward the Molecular Mechanism(s) by which EGCG Treatment Remodels Mature Amyloid Fibrils. J. Am. Chem. Soc. 135, 7503-7510 (2013).

30. Lopez del Amo, J. M. et al. Structural Properties of EGCG-Induced, Nontoxic Alzheimer's Disease A $\beta$ Oligomers. J. Mol. Biol. 421, 517-524 (2012).

31. Ehrnhoefer, D. E. et al. EGCG redirects amyloidogenic polypeptides into unstructured, offpathway oligomers. Nat. Struct. Mol. Biol. 15, 558-566 (2008).

32. $\mathrm{Xu}, \mathrm{Y}$. et al. Epigallocatechin Gallate (EGCG) Inhibits Alpha-Synuclein Aggregation: A Potential Agent for Parkinson's Disease. Neurochem. Res. 41, 2788-2796 (2016).

33. Wobst, H. J., Sharma, A., Diamond, M. I., Wanker, E. E. \& Bieschke, J. The green tea polyphenol (-)-epigallocatechin gallate prevents the aggregation of tau protein into toxic oligomers at substoichiometric ratios. FEBS Lett. 589, 77-83 (2015).

34. Ehrnhoefer, D. E. et al. Green tea (-)-epigallocatechin-gallate modulates early events in huntingtin misfolding and reduces toxicity in Huntington's disease models. Hum. Mol. Genet. 15, 2743-2751 (2006).

35. Yang, F. et al. Curcumin inhibits formation of amyloid beta oligomers and fibrils, binds plaques, and reduces amyloid in vivo. J. Biol. Chem. 280, 5892-5901 (2005).

36. Ono, K., Hasegawa, K., Naiki, H. \& Yamada, M. Curcumin has potent anti-amyloidogenic effects for Alzheimer's beta-amyloid fibrils in vitro. J. Neurosci. Res. 75, 742-750 (2004).

37. Pandey, N., Strider, J., Nolan, W. C., Yan, S. X. \& Galvin, J. E. Curcumin inhibits aggregation of alpha-synuclein. Acta Neuropathol. 115, 479-489 (2008).

38. Dikshit, P., Goswami, A., Mishra, A., Nukina, N. \& Jana, N. R. Curcumin enhances the polyglutamine-expanded truncated $\mathrm{N}$-terminal huntingtin-induced cell death by promoting proteasomal malfunction. Biochem. Biophys. Res. Commun. 342, 1323-1328 (2006).

39. Verma, M. et al. Curcumin Prevents Formation of Polyglutamine Aggregates by Inhibiting Vps36, a Component of the ESCRT-II Complex. PLoS ONE 7, e42923 (2012).

40. Burke, K. A., Hensal, K. M., Umbaugh, C. S., Chaibva, M. \& Legleiter, J. Huntingtin disrupts lipid bilayers in a polyQ-length dependent manner. Biochim. Biophys. Acta Biomembr. 1828, 1953-1961 (2013).

41. Chaibva, M. et al. Sphingomyelin and GM1 Influence Huntingtin Binding to, Disruption of, and Aggregation on Lipid Membranes. ACS Omega 3, 273-285 (2018). 
42. Gao, X. et al. Cholesterol Modifies Huntingtin Binding to, Disruption of, and Aggregation on Lipid Membranes. Biochemistry 55, 92-102 (2016).

43. Pandey, N. K. et al. The 17-residue-long $\mathrm{N}$ terminus in huntingtin controls stepwise aggregation in solution and on membranes via different mechanisms. J. Biol. Chem. 293, 25972605 (2018).

44. Legleiter, J., Fryer, J. D., Holtzman, D. M. \& Kowalewski, T. The Modulating Effect of Mechanical Changes in Lipid Bilayers Caused by ApoE-Containing Lipoproteins on A $\beta$ Induced Membrane Disruption. ACS Chem. Neurosci. 2, 588-599 (2011).

45. Yip, C. M., Darabie, A. A. \& McLaurin, J. Abeta42-peptide assembly on lipid bilayers. J. Mol. Biol. 318, 97-107 (2002).

46. Yip, C. M. \& McLaurin, J. Amyloid-beta peptide assembly: a critical step in fibrillogenesis and membrane disruption. Biophys. J. 80, 1359-1371 (2001).

47. Yates, E. A. et al. Specific Domains of A $\beta$ Facilitate Aggregation on and Association with Lipid Bilayers. J. Mol. Biol. 425, 1915-1933 (2013).

48. Jo, E., McLaurin, J., Yip, C. M., St George-Hyslop, P. \& Fraser, P. E. $\alpha$-Synuclein membrane interactions and lipid specificity. J. Biol. Chem. 275, 34328-34334 (2000).

49. Eliezer, D., Kutluay, E., Bussell, R. \& Browne, G. Conformational properties of $\alpha$-synuclein in its free and lipid-associated states11Edited by P. E. Wright. J. Mol. Biol. 307, 1061-1073 (2001).

50. Burke, K. A., Yates, E. A. \& Legleiter, J. Biophysical Insights into How Surfaces, Including Lipid Membranes, Modulate Protein Aggregation Related to Neurodegeneration. Front. Neurol. 4, (2013).

51. Kegel, K. B. et al. Huntingtin Associates with Acidic Phospholipids at the Plasma Membrane. J. Biol. Chem. 280, 36464-36473 (2005).

52. B, K.-G. K. Huntingtin Interactions with Membrane Phospholipids: Strategic Targets for Therapeutic Intervention? J. Huntingtons Dis. 239-250 (2013) doi:10.3233/JHD-130068.

53. Lee, W.-C. M., Yoshihara, M. \& Littleton, J. T. Cytoplasmic aggregates trap polyglutaminecontaining proteins and block axonal transport in a Drosophila model of Huntington's disease. Proc. Natl. Acad. Sci. U.S.A. 101, 3224-3229 (2004).

54. Gauci, A. J., Caruana, M., Giese, A., Scerri, C. \& Vassallo, N. Identification of polyphenolic compounds and black tea extract as potent inhibitors of lipid membrane destabilization by $\mathrm{A} \beta$ aggregates. J. Alzheimers dis. (2011) doi:10.3233/JAD-2011-111061.

55. Caruana, M. et al. Inhibition and disaggregation of $\alpha$-synuclein oligomers by natural polyphenolic compounds. FEBS Lett. 585, 1113-1120 (2011). 
56. Camilleri, A. et al. Mitochondrial membrane permeabilisation by amyloid aggregates and protection by polyphenols. Biochim. Biophys. Acta 1828, 2532-2543 (2013).

57. Malishev, R. et al. Toxicity Inhibitors Protect Lipid Membranes from Disruption by A $\beta 42$. ACS Chem. Neurosci. 6, 1860-1869 (2015).

58. Muchowski, P. J. et al. Hsp70 and Hsp40 chaperones can inhibit self-assembly of polyglutamine proteins into amyloid-like fibrils. Proc. Natl. Acad. Sci. U.S.A. 97, 7841-7846 (2000).

59. Burke, K. A., Godbey, J. \& Legleiter, J. Assessing mutant huntingtin fragment and polyglutamine aggregation by atomic force microscopy. Methods 53, 275-284 (2011).

60. Burke, K. A. \& Legleiter, J. Atomic Force Microscopy Assays for Evaluating Polyglutamine Aggregation in Solution and on Surfaces. in Tandem Repeats in Genes, Proteins, and Disease: Methods and Protocols (eds. Hatters, D. M. \& Hannan, A. J.) 21-40 (Humana Press, 2013). doi:10.1007/978-1-62703-438-8_2.

61. Zheng, F., Wu, Z. \& Chen, Y. A quantitative method for the measurement of membrane affinity by polydiacetylene-based colorimetric assay. Anal. Biochem. 420, 171-176 (2012).

62. Sokolovski, M., Sheynis, T., Kolusheva, S. \& Jelinek, R. Membrane interactions and lipid binding of casein oligomers and early aggregates. Biochim. Biophys. Acta Biomembr 1778, 2341-2349 (2008).

63. Chaibva, M. et al. Acetylation within the First 17 Residues of Huntingtin Exon 1 Alters Aggregation and Lipid Binding. Biophys. J. 111, 349-362 (2016).

64. Burke, K. A., Kauffman, K. J., Umbaugh, C. S., Frey, S. L. \& Legleiter, J. The Interaction of Polyglutamine Peptides with Lipid Membranes Is Regulated by Flanking Sequences Associated with Huntingtin. J. Biol. Chem. 288, 14993-15005 (2013).

65. Burke, K. A., Yates, E. A. \& Legleiter, J. Amyloid-Forming Proteins Alter the Local Mechanical Properties of Lipid Membranes. Biochemistry 52, 808-817 (2013).

66. Atwal, R. S. et al. Huntingtin has a membrane association signal that can modulate huntingtin aggregation, nuclear entry and toxicity. Hum. Mol. Genet. 16, 2600-2615 (2007).

67. Gauthier, L. R. et al. Huntingtin controls neurotrophic support and survival of neurons by enhancing BDNF vesicular transport along microtubules. Cell 118, 127-138 (2004).

68. Gunawardena, S. et al. Disruption of axonal transport by loss of huntingtin or expression of pathogenic polyQ proteins in Drosophila. Neuron 40, 25-40 (2003).

69. Pal, A., Severin, F., Lommer, B., Shevchenko, A. \& Zerial, M. Huntingtin-HAP40 complex is a novel Rab5 effector that regulates early endosome motility and is up-regulated in Huntington's disease. J. Cell Biol. 172, 605-618 (2006). 
70. Suopanki, J. et al. Interaction of huntingtin fragments with brain membranes - clues to early dysfunction in Huntington's disease. J. Neurochem. 96, 870-884 (2006).

71. De Rooij, K. E., Dorsman, J. C., Smoor, M. A., Den Dunnen, J. T. \& Van Ommen, G. J. Subcellular localization of the Huntington's disease gene product in cell lines by immunofluorescence and biochemical subcellular fractionation. Hum. Mol. Genet. 5, 10931099 (1996).

72. Saudou, F., Finkbeiner, S., Devys, D. \& Greenberg, M. E. Huntingtin acts in the nucleus to induce apoptosis but death does not correlate with the formation of intranuclear inclusions. Cell 95, 55-66 (1998).

73. Trettel, F. et al. Dominant phenotypes produced by the HD mutation in STHdhQ111 striatal cells. Hum. Mol. Genet. 9, 2799-2809 (2000).

74. Xia, J., Lee, D. H., Taylor, J., Vandelft, M. \& Truant, R. Huntingtin contains a highly conserved nuclear export signal. Hum. Mol. Genet. 12, 1393-1403 (2003).

75. Atwal, R. S. et al. Kinase inhibitors modulate huntingtin cell localization and toxicity. Nat. Chem. Biol. 7, 453-460 (2011).

76. Chang, D. T. W., Rintoul, G. L., Pandipati, S. \& Reynolds, I. J. Mutant huntingtin aggregates impair mitochondrial movement and trafficking in cortical neurons. Neurobiol. Dis. 22, 388400 (2006).

77. Choo, Y. S., Johnson, G. V. W., MacDonald, M., Detloff, P. J. \& Lesort, M. Mutant huntingtin directly increases susceptibility of mitochondria to the calcium-induced permeability transition and cytochrome c release. Hum. Mol. Genet. 13, 1407-1420 (2004).

78. Gu, M. et al. Mitochondrial defect in Huntington's disease caudate nucleus. Ann. Neurol. 39, 385-389 (1996).

79. Orr, A. L. et al. N-Terminal Mutant Huntingtin Associates with Mitochondria and Impairs Mitochondrial Trafficking. J. Neurosci. 28, 2783-2792 (2008).

80. Panov, A. V. et al. Early mitochondrial calcium defects in Huntington's disease are a direct effect of polyglutamines. Nat. Neurosci. 5, 731-736 (2002).

81. Levy, G. R. et al. Huntingtin's N-Terminus Rearrangements in the Presence of Membranes: A Joint Spectroscopic and Computational Perspective. ACS Chem. Neurosci. 10, 472-481 (2019).

82. Chaibva, M., Burke, K. A. \& Legleiter, J. Curvature Enhances Binding and Aggregation of Huntingtin at Lipid Membranes. Biochemistry 53, 2355-2365 (2014).

83. Rao, P. P. N., Mohamed, T., Teckwani, K. \& Tin, G. Curcumin Binding to Beta Amyloid: A Computational Study. Chem. Biol. Drug Des. 86, 813-820 (2015). 
84. Martin, T. D., Malagodi, A. J., Chi, E. Y. \& Evans, D. G. Computational Study of the Driving Forces and Dynamics of Curcumin Binding to Amyloid- $\beta$ Protofibrils. J. Phys. Chem. B 123, 551-560 (2019).

85. Engel, M. F. M. et al. The Polyphenol EGCG Inhibits Amyloid Formation Less Efficiently at Phospholipid Interfaces than in Bulk Solution. J. Am. Chem. Soc. 134, 14781-14788 (2012). 


\section{Lipid headgroups alter huntingtin aggregation on membranes}

Huntington's Disease is a neurodegenerative disorder caused by an expanded polyglutamine(polyQ) domain within the huntingtin protein (htt). As a consequence of polyQ expansion, htt associates into a variety of aggregates that underlie cellular toxicity. Within cells, htt associates with numerous membranous organelles that exert influence on the aggregation process. In particular, the first 17 amino acids at the N-terminus of htt (Nt17) serve as a lipidbinding domain that is intrinsically disordered in bulk solution but adopts an amphipathic $\alpha$ helical structure upon binding membranes. Beyond this, Nt17 is implicated in initiating htt fibrillization. As the htt/membrane interaction is likely influenced by lipid properties, the impact of lipid headgroups on htt-exon1 aggregation, membrane activity, and the ability to form protein:lipid complexes was determined. Htt-exon1 with a disease-length polyQ domain was exposed to lipid vesicles comprised of lipids with either zwitterionic (POPC and POPE) or anionic (POPG and POPS) headgroups. With zwitterionic head groups, large lipid to peptide ratios were required to have a statistically significant impact on htt aggregation. Anionic lipids enhanced htt fibrillization, even at low lipid:protein ratios, and this altered aggregate morphology. Despite the larger impact of anionic lipids, htt-exon1(46Q) was more membrane active with zwitterionic lipid systems. The ability of Nt17 to form complexes with lipids was mediated by lipid headgroups as zwitterionic ionic lipids more readily associated with multimeric forms of Nt17 in comparison with anionic lipids. These results highlight the complexity of htt/membrane interactions and the resulting impact on the aggregation process. 


\subsection{Introduction}

Amyloid diseases are defined by abnormal deposition of proteinaceous aggregates (amyloid fibrils or plaques) in tissues and organs. Amyloid forming proteins include $\beta$-amyloid $(\mathrm{A} \beta)$ and tau related with Alzheimer's disease (AD), $\alpha$-synuclein ( $\alpha$-syn) implicated in Parkinson's disease (PD), human islet amyloid polypeptide (hIAPP) linked with type II diabetes, and the huntingtin protein (htt) associated with Huntington's disease (HD). In general, amyloid fibrils form via a nucleus-dependent polymerization mechanism beginning with a rate-limiting nucleation step that leads to rapid elongation of the $\beta$-sheet rich aggregates. ${ }^{1-3}$ In HD, a genetic neurodegenerative disorder, an aberrant expansion of a polyglutamine (polyQ) region in the first exon of htt ( $>35$ repeat glutamine residues) promotes toxic aggregation. Beyond the formation of fibrils, htt aggregates via numerous mechanisms that can occur simultaneously, resulting in a heterogeneous mixture of species, including oligomers, fibrils, annular aggregates, and inclusion bodies. $^{4-6}$

The complexity of htt aggregation makes it difficult to gain consensus on the prominent aggregate form involved in toxicity. Several aggregate species could be involved in a variety of potential cytotoxic mechanisms, while others may impart a protective effect. Inclusion bodies were initially thought to be prominent toxic entities as their formation preceded behavioral deficits in mouse models. ${ }^{7}$ While it is true that large scale cellular degeneration is observed in the striatum coinciding with the presence of intracellular aggregates comprised of mutant htt, there is only moderate degeneration within the cerebral cortex despite a typically larger inclusion load. ${ }^{8}$ A poor correlation between inclusion body formation and toxicity is also apparent in cellular models of HD. ${ }^{9}$ Survival analysis suggests that particularly potent toxic species reside in diffusely distributed htt throughout cells and that the formation of inclusion bodies may be 
protective. ${ }^{10}$ However, the diffuse htt population implicated in toxicity is a complex mixture that includes htt species ranging from monomers and small oligomers ${ }^{11-13}$ to amorphous aggregates and fibrils, ${ }^{14-16}$ making it difficult to pinpoint toxic species. In fact, there is evidence linking htt oligomers, ${ }^{12,17,18}$ fibrils, ${ }^{19,20}$ and even inclusions ${ }^{21}$ to cellular dysfunction and toxicity.

While aggregation is a complex process, the direct interaction of htt with intracellular and subcellular membranes that are predominately comprised of lipids further modifies aggregation. For example, the first N-terminal 17 amino acids (Nt17) of htt-exon1 is implicated in promoting the formation of $\alpha$-helix rich oligomer intermediates that nucleate fibril formation; however, Nt17 facilitates the binding of htt to lipid membranes by forming an amphipathic $\alpha$-helix. A number of model lipid membranes, ranging from total brain lipid extracts (TBLE) to pure lipid systems, alter the rate of aggregation and the resulting aggregate species observed. For example, POPC/POPS lipid vesicles enhanced fibril formation, ${ }^{22}$ but TBLE and other brain extracts tend to reduce fibrillization. ${ }^{23}$ In addition, lipid components ranging from cholesterol, ${ }^{24}$ sphingomyelin, and $\mathrm{GM} 1^{25}$ can further modify htt aggregation on membranes.

Beyond influencing aggregation, several toxic mechanisms involve htt/lipid interactions. Htt aggregation on membranes can lead to membrane disruption and permeabilization that has been associated with organelle dysfunction in HD. ${ }^{26-29}$ In addition, mutant htt demonstrates enhanced binding to phospholipid bilayers compared to wild type $\mathrm{e}^{30-32}$ and is associated with membranous organelles such as the endoplasmic reticulum (ER), ${ }^{33}$ mitochondria, ${ }^{34,35}$ and golgi apparatus. ${ }^{36} \mathrm{Htt}$ aggregates accumulate brain lipids ${ }^{27,37}$ and membranous structures are incorporated into htt inclusion bodies. ${ }^{38,39}$ The association of htt with organelles via membrane interactions result in disrupted trafficking, ${ }^{34}$ synaptic degeneration, ${ }^{40}$ and neuronal cell death. ${ }^{41}$ While posttranslational modifications within Nt17 regulate the localization of htt to different 
organelles, the membrane composition of these organelles also plays a role, as membrane physicochemical properties dictate protein/lipid interactions.

Here, the aim was to determine how lipid headgroups influence the membrane activity of htt. To achieve this, the extent of aggregation and membrane activity of htt in the presence of model lipid vesicles with identical tails but different headgroups (POPC, POPE, POPG, POPS) was determined. To further investigate the role of surface charge, two of the selected systems are zwitterionic (POPC and POPE), while two are anionic (POPG and POPS). First, the impact of the different lipid systems on the rate of htt aggregation and resulting aggregate morphology was elucidated with thioflavin-T (ThT) aggregation assays and atomic force microscopy (AFM). Htt membrane activity and protein/lipid complex formation were resolved via polydiacetylene (PDA) lipid binding assays and native mass spectrometry (MS). These studies suggest that htt membrane activity increases with bilayer fluidity and that increased membrane charge density promotes a unique lipid interaction mechanism that alters oligomer formation and enhances fibrilization.

\subsection{Materials and methods}

\subsubsection{Purification of glutathione S-transferase (GST)-htt exon1 fusion protein}

Glutathione S-transferase (GST)-htt exon1 fusion proteins with pathogenic-length polyglutamine repeats (46Q) were expressed and purified as previously described. ${ }^{42}$ Briefly, the protein was expressed in Escherichia coli for $4 \mathrm{~h}$ at $37^{\circ} \mathrm{C}$ followed by the addition of lysozyme and probe-sonication to release the protein. Purification was accomplished using a BioRad low pressure liquid chromatograph equipped with a GST-affinity column. Relevant fractions were pooled into one bulk solution and placed in dialysis at $4{ }^{\circ} \mathrm{C}$ for 2 days. Prior to any experiments, 
the protein solution was subjected to a high-speed centrifugation $(22,000 \times \mathrm{g}$ for $30 \mathrm{~min})$ to remove any aggregate species that may have formed during dialysis. To initiate aggregation, the desired volume of protein solution was incubated with Factor Xa (Promega, Madison, WI) for 30 min to cleave the GST tag from the htt-exon1(46Q). All experiments were carried out in a tris buffer (150 mM NaCl, Tris-HCl, pH 7.4).

\subsubsection{Formation of lipid vesicles}

Lyophilized lipid was dissolved in chloroform and aliquoted into Eppendorf tubes. The aliquots were dried under a gentle stream of nitrogen, and the resulting lipid films were stored at $-20{ }^{\circ} \mathrm{C}$ until needed. For experiments, lipid films were rehydrated in tris buffer $(150 \mathrm{mM} \mathrm{NaCl}$, Tris-HCl, $\mathrm{pH} 7.4$ ) for $1 \mathrm{~h}$ at $30^{\circ} \mathrm{C}$. Once rehydrated, the lipids were subjected to 10 freeze-thaw cycles using liquid nitrogen and a thermomixer $\left(55^{\circ} \mathrm{C}\right)$. After the freeze-thaw cycles, lipid solutions were bath-sonicated for $1 \mathrm{~h}$ and then stored at $4{ }^{\circ} \mathrm{C}$.

\subsubsection{Thioflavin $T$ aggregation assay}

Thioflavin T fluorescence assays (ThT, Sigma-Aldrich, St. Louis, MO) were performed to monitor fibril formation. To ensure GST cleavage, htt-exon1(46Q) was incubated with cleaving agent Factor Xa for $1 \mathrm{~h}$ on ice. Then, htt-exon1(46Q) $(20 \mu \mathrm{M})$ was incubated with ThT $(200 \mu \mathrm{M})$ in the presence and absence of various pure lipid systems (POPC, POPE, POPG, or POPS). Prior to the addition of ThT or lipid vesicles, the htt-exon1(46Q) underwent centrifugation to remove preexisting aggregates. When lipid was present, the concentration was either $100 \mu \mathrm{M}, 200 \mu \mathrm{M}$, or $400 \mu \mathrm{M}$ to result in lipid:htt ratios of 5:1, 10:1, or 20:1. Experiments were performed in Costar clear-bottom 96 -well plates at $37^{\circ} \mathrm{C}$ and $\mathrm{ThT}$ fluorescence was monitored with a SpetraMax M2 microplate reader (440 nm excitation, $484 \mathrm{~nm}$ emission) every 
$5 \mathrm{~min}$ over an $18 \mathrm{~h}$ period. Each experiment was performed three separate times, and within each experiment individual conditions were run in triplicate. Reported values are an average of the three trials within one representative experiment used in figures. The rate was calculated as previously described. ${ }^{23}$ Briefly, the rate was measured over a $3 \mathrm{~h}$ period that started when the intensity of the fluorescence exceeded $10 \%$ of the maximum signal obtained for that condition. This threshold was selected to ensure a significant increase in fluorescence and to avoid calculation starting in any noise in the lag phase. Both the rate and the maximum fluorescence for each condition were normalized to the htt control (100\%).

\subsubsection{Atomic force microscopy}

Ex situ atomic force microscopy (AFM) was used to investigate the morphology of htt aggregates formed in the presence or absence of lipid vesicles. Htt-exon1(46Q) $(20 \mu \mathrm{M})$ was incubated with and without $400 \mu \mathrm{M}$ lipid vesicles (to result in a $20: 1$ lipid:protein ratio) at $37^{\circ} \mathrm{C}$ for a period of $8 \mathrm{~h}$. After 1,3,5, and $8 \mathrm{~h}$ of incubation, a $2 \mu \mathrm{L}$ aliquot of each condition was deposited on freshly cleaved mica for $1 \mathrm{~min}$. Then, the mica was rinsed with $18 \mathrm{M} \Omega$ water and dried with a gentle stream of clean air. Samples were imaged using a Nanoscope V Multi-Mode scanning probe microscope (VEECO) equipped with a closed-loop vertical engage J-scanner. Silicon-oxide cantilevers with nominal spring constant of $40 \mathrm{~N} / \mathrm{m}$ and a resonance frequency of $300 \mathrm{kHz}$ were used. Scan rates were set to $1.99 \mathrm{~Hz}$ with cantilever drive frequencies at $10 \%$ off resonance. All images were analyzed using the Matlab image processing toolbox (MathWorks) as previously described. ${ }^{5,43}$ 


\subsubsection{Polydiacetylene lipid binding assay}

A normalized polydiacetylene (PDA) lipid binding assay was utilized to measure the interaction between htt-exon1(46Q) and different lipid systems. Briefly, diacetylene monomers (10,12-tricosadiynoic acid) and various lipid systems were dissolved in a 4:1 chloroform:ethanol solution and mixed at a 2:3 molar ratio. Solutions were dried under a gentle stream of nitrogen until a fully dried lipid film was formed, and then films were reconstituted in hot tris buffer (150 $\mathrm{mM} \mathrm{NaCl}$, Tris- $\mathrm{HCl}, \mathrm{pH} \mathrm{7.4,} 70{ }^{\circ} \mathrm{C}$ ). Lipid solutions were sonicated at $125 \mathrm{~W}$ for $10 \mathrm{~min}$, then protected from light and left at $4{ }^{\circ} \mathrm{C}$ overnight to allow vesicle formation. The following day, lipid vesicles were exposed to UV light $(254 \mathrm{~nm})$ to polymerize the diacetylene monomers, resulting in a royal blue solution that can undergo a colorimetric shift to red as a result of mechanical stress. PDA/lipid solutions $(400 \mu \mathrm{M})$ were incubated with htt-exon1(46Q) at $30{ }^{\circ} \mathrm{C}$, and the blue $(650 \mathrm{~nm})$ and red $(500 \mathrm{~nm})$ absorbances were recorded every $5 \mathrm{~min}$ for $18 \mathrm{~h}$. The negative control consisted of equal volumes of neat buffer and PDA/lipid solution, while the internal standard involved equal volumes of $1 \mathrm{M} \mathrm{NaOH}$ and PDA/lipid. The $\mathrm{NaOH}$ creates repulsion between headgroups via protonation, causing a colorimetric shift. The dose of $\mathrm{NaOH}$ used saturates the system, resulting in a maximum colorimetric shift for each lipid system that can be used to normalize results. The $\% \mathrm{CR}$ was calculated for each condition using the following equation:

$$
\% C R=\left(\frac{P B_{0}-P B}{P B_{0}}\right) \times 100
$$

where $\mathrm{PB}$ is defined as $\mathrm{A}_{\text {blue }} /\left(\mathrm{A}_{\text {blue }}+\mathrm{A}_{\text {red }}\right)$ for the negative control $\left(\mathrm{PB}_{0}\right)$ and sample condition (PB). 


\subsubsection{Electrospray Ionization-Mass Spectrometry (ESI-MS)}

Native $\mathrm{MS}^{44}$ was used to investigate complexes formed between Nt17 peptide and various lipid vesicles. ${ }^{45}$ Lipid vesicles were formed as previously described, but films were reconstituted in HPLC-grade water instead of tris buffer. Nt17 peptide $(20 \mu \mathrm{M})$ was incubated with lipid vesicles (20:1 lipid:peptide) for $5 \mathrm{~h}$ at $37^{\circ} \mathrm{C}$. Samples were analyzed using a Q Exactive Hybrid Quadrupole mass spectrometer (Thermo Fischer, San Jose, CA) equipped with a commercial HESI source. Spectra were recorded in positive-ion mode over a mass-to-charge $(\mathrm{m} / \mathrm{z})$ ratio range of 400 to 4,000 , and samples that included anionic lipids were also recorded in negative-ion mode. Samples were infused at a rate of $10 \mathrm{uL} / \mathrm{min}$ and the needle was biased at 3,500 V relative to the mass spectrometer inlet. The parameters for the MS instrument were: 400 ${ }^{\circ} \mathrm{C}$ for the capillary inlet temperature, $30{ }^{\circ} \mathrm{C}$ for the analyzer temperature, $80 \mathrm{~V}$ for the $\mathrm{S}$-lens assembly, $400 \mathrm{~ms}$ for the maximum injection time, $1 \times 10^{6}$ for the AGC, and 70,000 for the MS resolution. Each spectrum was recorded in triplicate for $90 \mathrm{~s}$ each, and the data was analyzed using the Xcalibur 2.2 software suite (Thermo Scientific).

\subsection{Results}

\subsubsection{Lipids modify htt aggregation to various extents}

All experiments in this study, with exception of the native MS studies described later, were performed with a mutant htt-exon1 fragment with 46Q (htt-exon1(46Q)). Htt-exon1(46Q) was expressed as a soluble fusion with GST, and cleavage of GST by Factor Xa initiates aggregation. Htt-exon1(46Q) was incubated with vesicles of POPC, POPE, POPG, or POPS at various lipid to protein molar ratios (5:1, 10:1, and 20:1), and aggregation was monitored as a function of time using ThT, which fluoresces when bound to $\beta$-sheet structure associated with 
fibrils (Fig. 4.1a-d). All relevant controls were measured and subtracted from the curves to show only the fluorescence signal from htt aggregation. Htt aggregation in the absence of lipids was performed for comparison. Multiple independent experiments were performed. The slope of the ThT curves were analyzed to compare the relative rates of aggregation for each condition (Fig. 4.1e), as was the maximum signal for each condition to compare the extent of fibril formation (Fig. 4.1f).

The effect of lipid vesicles on htt-exon1(46Q) aggregation appeared to be greatly dependent on the headgroup charge of each lipid system. The two zwitterionic lipid systems POPC and POPE - each had minimal impact on htt-exon1(46Q) aggregation until the 20:1 lipid:protein ratio (Fig. 4.1a-b). At 5:1 or 10:1 lipid:protein ratio, neither lipid system resulted in a change to the maximum fluorescence of more than $\pm 5 \%$ when compared to the control. In the presence of 20:1 POPC, the rate of htt-exon1(46Q) fibrilization increased by $39 \%$ and resulted in $44 \%$ increase in the average ThT signal compared to the absence of lipid (Fig. 4.1e-f). However, the presence of 20:1 POPE reduced the aggregation rate by $18 \%$, which resulted in a $24 \%$ decrease in the maximum ThT signal compared to control (Fig. 4.1e-f).

While the two zwitterionic lipid systems ultimately influenced htt aggregation differently (POPC enhanced aggregation; POPE inhibited aggregation), statistically significant changes were not observed until the lipid to protein ratio became sufficiently large. In contrast, the two anionic lipid systems (POPG and POPS) significantly enhanced the rate and extent of htt aggregation at lipid to protein ratios as small as 5:1 (Fig. 4.1c-d). For both anionic lipid systems, the largest increase in aggregate formation occurred at the 5:1 lipid:protein ratio (114\% increase with POPG and 66\% increase with POPS compared to htt aggregation in the absence of lipids). Increasing the lipid:protein ratio resulted in a decrease in the effect for lipid systems. The 
presence of POPG vesicles increased the extent of aggregation by $106 \%$ at the $10: 1$ ratio, but this decreased to an $84 \%$ increase at the $20: 1$ ratio. The same trend appeared with POPS, where adding more lipid decreased the impact on the extent of aggregation (62\% increase at 10:1 and $38 \%$ increase at 20:1). For both systems, changes in the rate of aggregation mirrored the extent of aggregation (Fig. 4.1e-f). POPG increased the rate of aggregation formation at all concentrations (185\% increase at 5:1 and 10:1, and 143\% increase at 20:1), as did POPS (232\% increase at 5:1,126\% increase at 10:1, and $70 \%$ increase at $20: 1)$.

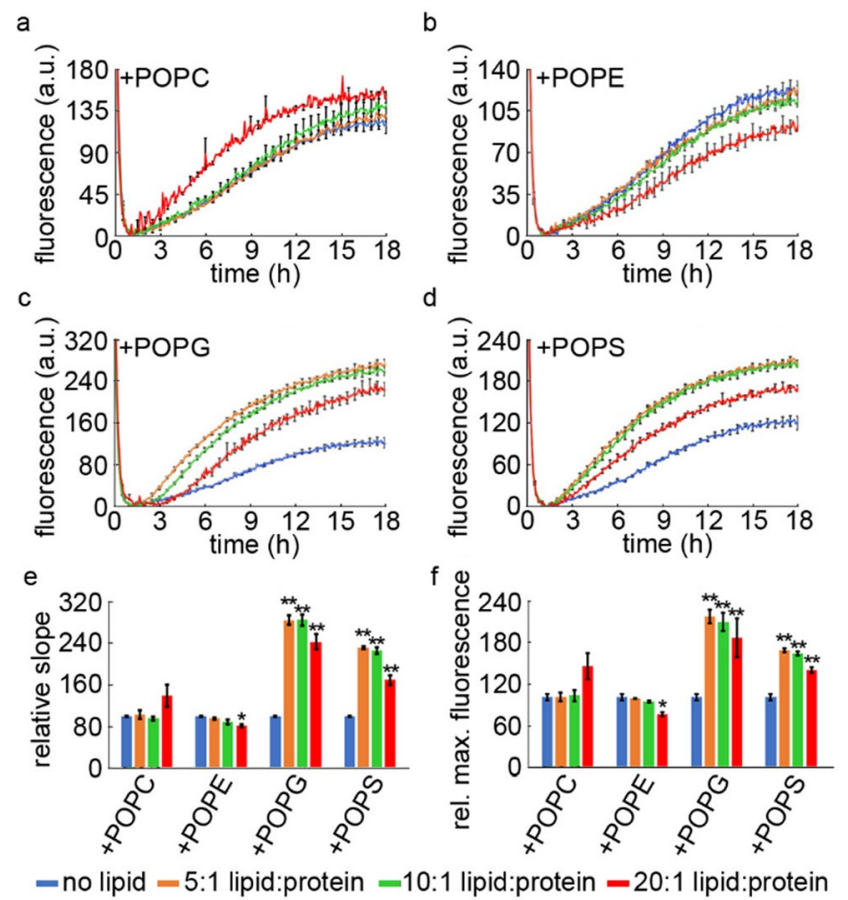

$=$ no lipid $=5: 1$ lipid:protein $=10: 1$ lipid:protein $=20: 1$ lipid:protein
Figure 4.1 Varying the lipid headgroup alters how vesicles influence htt fibrilization. ThT aggregation assays for htt-exon1(46Q) in the presence of (a) POPC, (b) POPE, (c) POPG, or (d) POPS lipid vesicles. Htt concentration was 20 $\mu \mathrm{M}$, and the lipid to htt molar ratio was $5: 1,10: 1$, or $20: 1$. The aggregation rate for each condition is represented by the (e) slope relative to the control assay with no lipids, which is set to 100 . The (f) relative maximum fluorescence for each condition was calculated in the same way, setting the no lipid condition to 100 . In panels a-d, error bars are provided for every sixth data point $(30 \mathrm{~min}$ ) and represent the standard error of the mean (SEM). Analyses shown in panels e and $\mathrm{f}$ were determined as averages over all trials, and values are normalized as a percentage with respect to the htt control in the absence of lipid. Error bars represent SEM. * represents a $p$ value $<$ 0.05 , and $* *$ represent a $p$ value $<0.01$ using a Student's t-test. 
To further investigate the influence that the different lipid vesicles have on httexon1(46Q) aggregation, AFM analysis was performed (Fig. 4.2). Incubation experiments were performed with monomeric htt-exon1(46Q) $(20 \mu \mathrm{M})$ in the presence and absence of POPC, POPE, POPG, or POPS lipid vesicles at a molar ratio of 20:1 lipid:htt. At designated time points $(1,3,5$, and $8 \mathrm{~h})$, incubations were sampled for AFM analysis. Control incubations of the different lipid vesicles were also imaged to determine the associated backgrounds on the mica surface (Fig. 4.3). For all incubation conditions, a variety of oligomers and fibrils were observed (Fig. 4.2), and further analysis of aggregate population and morphology was carried out. To quantify the effect of the lipids on aggregation, AFM images from all incubations were analyzed by counting the number of fibrils and oligomers per square micron using automated scripts written in Matlab that determined morphological features of each individual aggregate present in the image. For the analysis, oligomers were classified as any feature taller than $1.0 \mathrm{~nm}$ with an area of fewer than 40 pixels and with an aspect ratio (longest distance across to shortest distance across) of less than 3.0, indicating a globular structure. The $1.0 \mathrm{~nm}$ height threshold was selected to overcome the effect of background associated with AFM images of lipid controls. Fibrils were identified and counted by hand in each image. 

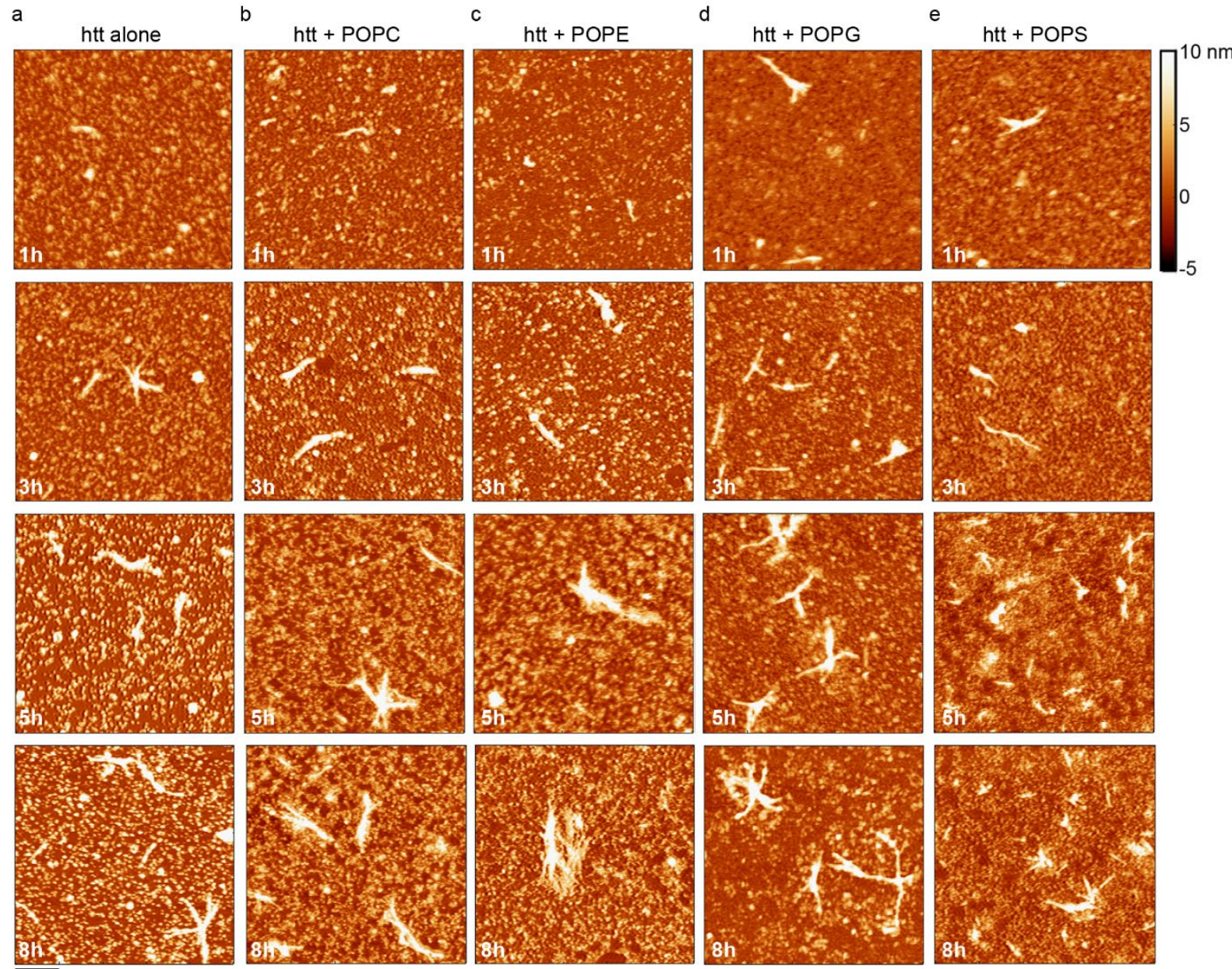

Figure 4.2 Representative AFM images showing the effect of different lipids on htt-

exon1 (46Q) aggregation. Htt-exon1(46Q) $(20 \mu \mathrm{M})$ was incubated with (a) no lipid, (b) POPC vesicles, (c) POPE vesicles, (d) POPG vesicles, or (e) POPS vesicles at various time points. The ratio of lipid to htt was 20:1. The colormap and scale bar is applicable to all images. 

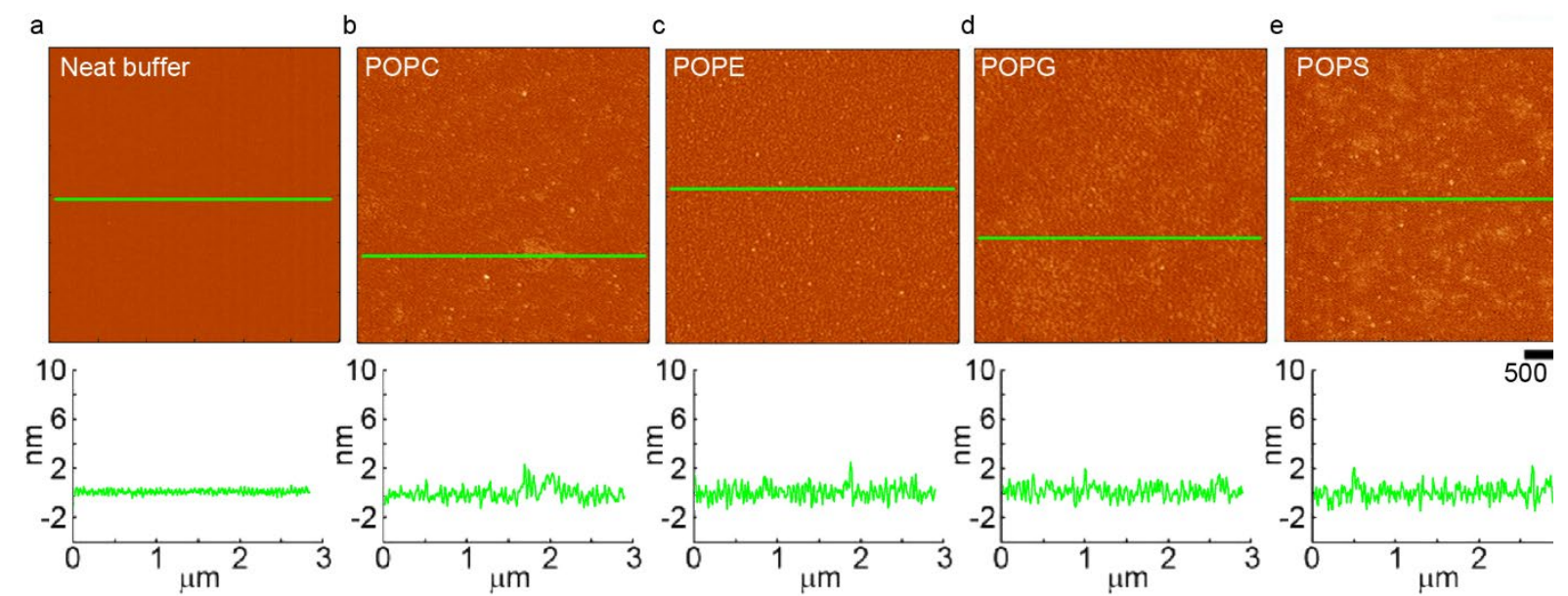

Figure 4.3 AFM images of lipid backgrounds. Representative AFM images of the backgrounds associated with (a) neat buffer, (b) POPC, (c) POPE, (d) POPG, and (e) POPS lipid vesicles deposited on mica. The line in each image corresponds to the height profile provided below each image. These backgrounds were used to determine appropriate height threshold values for AFM image analysis.

Consistent with the ThT assays, each lipid system (POPC, POPE, POPG, POPS) impacted the extent of htt-exon1(46Q) fibrilization. In the absence of lipid, a small population of fibrils were present after $1 \mathrm{~h}$, and fibril content grew steadily with time (Figs. 4.2 and 4.4a). While ThT fluorescence is traditionally interpreted to be a measure of fibril formation, raw fibril counts aren't necessarily directly comparable to ThT assays as the length of fibrils varies greatly and fibril bundling can occur. Therefore, the total extent of fibril formation was also determined at the $8 \mathrm{~h}$ time point by measuring the percent of the surface area covered by fibrils (Fig. 4.4b). After $8 \mathrm{~h}$ of incubation, fibrils covered $\sim 2 \%$ of the mica surface. As fibril thickness can vary along its length, the fibril morphology was assessed by measuring the average height along the contour, and the mode fibril height was $7.5 \mathrm{~nm}$ (Fig. 4.4c). 


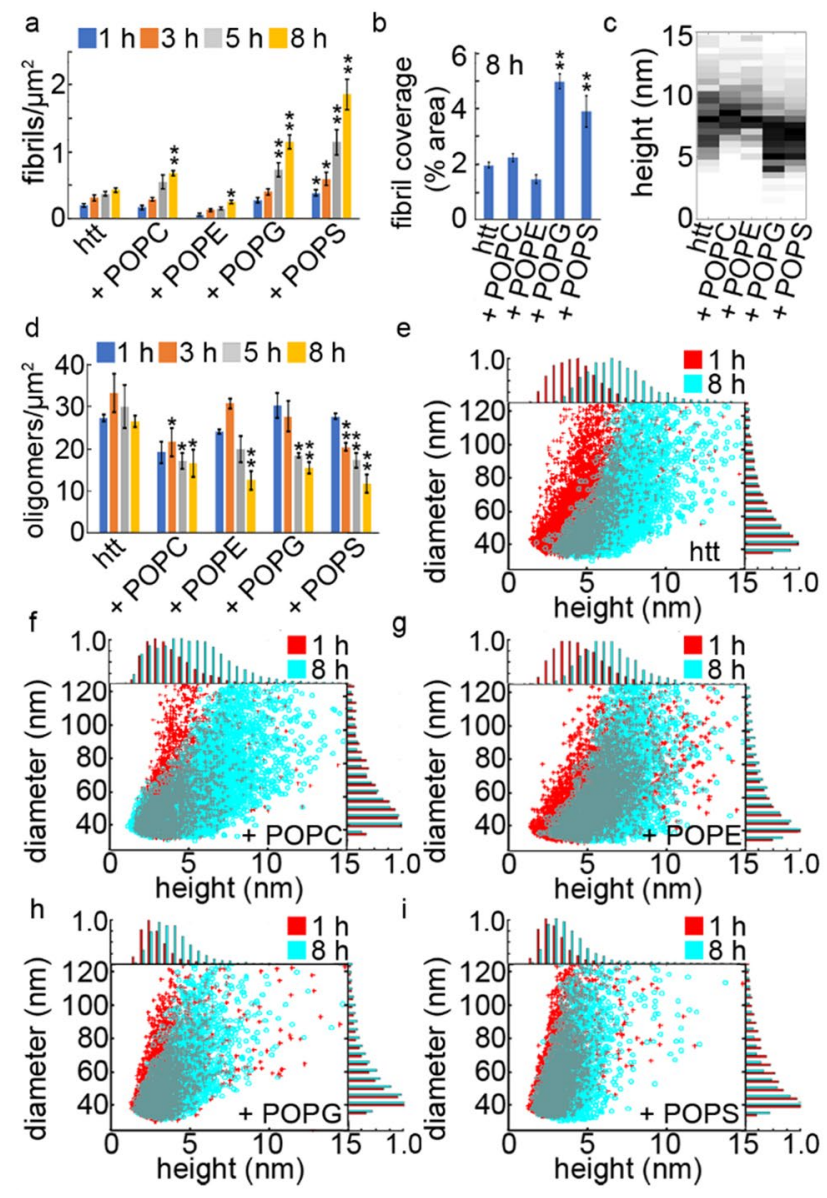

Figure 4.4 AFM analysis of the impact of different lipid systems on httexon1(46Q) aggregation. (a) Analysis of the number of fibrils formed in the presence of each lipid system as a function of time. (b) The percent area of each image covered by fibrils at the $8 \mathrm{~h}$ time point for each condition. (c) Height histograms of fibrils formed in the presence of different lipid systems. (d) Image analysis of oligomers per unit area as a function of time for each condition. Correlation plots of the height and diameter of each oligomer and histograms of these morphological features at $1 \mathrm{~h}$ and $8 \mathrm{~h}$ in the presence of (e) no lipid, (f) POPC, (g) POPE, (h) POPG, and (i) POPS vesicles. For panels a, b, and d, error bars represent the SEM, * represents a $\mathrm{p}$ value $<0.05$, and $* *$ represent a $p$ value $<0.01$ using a Student's t-test.

In the presence of POPC lipid vesicles, the number of htt-exon1(46Q) fibrils was similar to control during the first $5 \mathrm{~h}$ but there was a statistically significant increase in fibril population after $8 \mathrm{~h}(\mathrm{p}<0.01$, Fig. $4.4 \mathrm{a})$. Despite this increased number of fibrils, fibril content formed in the presence of POPC vesicles covered only a slightly larger surface area compared with control (2.3\%, Fig. 4.4b). The mode height $(8.0 \mathrm{~nm})$ of fibrils formed with POPC vesicles was similar to control (Fig. 4.4c). In the presence of POPE vesicles, htt-exon1(46Q) formed fewer fibrils than control at all time points (Fig. 4.4a) and covered a smaller surface area (1.5\%, Fig. 4.4b). Again, fibril morphology was indistinguishable from control as the mode height was $7.5 \mathrm{~nm}$ (Fig. 4.4c). 
With both anionic lipid systems (POPG and POPS), the number of fibrils formed was significantly larger compared with control (Fig. 4.4a), with the largest fibril population observed with POPS. Under both POPG and POPS conditions, fibrils covered a significantly larger surface area at $8 \mathrm{~h}$ (5.0\% for POPG and $3.9 \%$ for POPS, Fig. $4.4 \mathrm{~b})$. The fibril morphology was also altered by anionic lipids, as the distribution and mode height of these fibrils was shifted to smaller values with heights mainly ranging from 5-7.5 $\mathrm{nm}$ (Fig. 4.4c). In particular, fibrils formed with POPG developed a unique morphology with time. That is, fibrils bundled into clumps with multiple fibrillar extensions emanating from a central core (Fig. 4.5). Fibrils formed with POPS vesicles were shorter in length than control, as demonstrated by the number of fibrils formed being $\sim 4 \times$ that of control while only covering $\sim 2 \times$ the surface area.

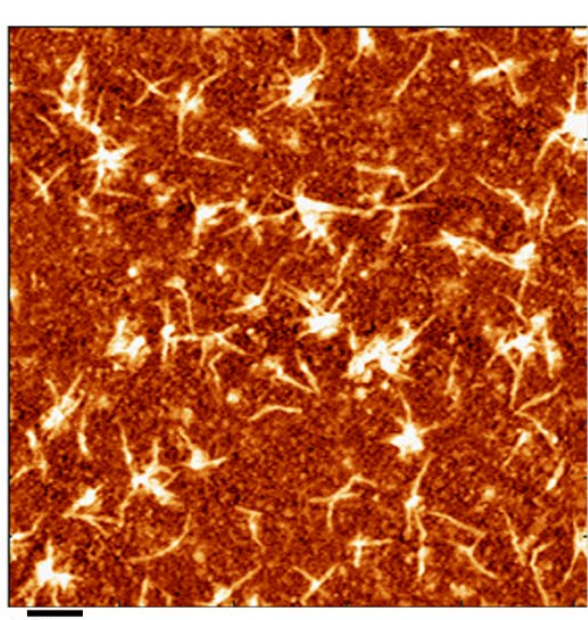

$500 \mathrm{~nm}$
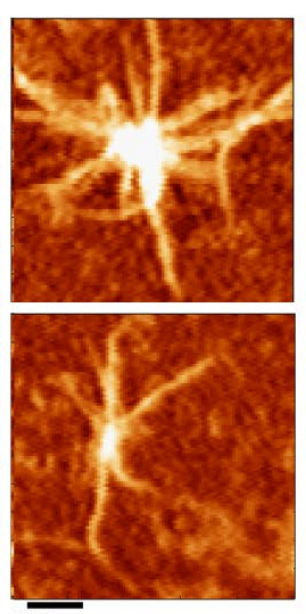

$\overline{200 \mathrm{~nm}}$
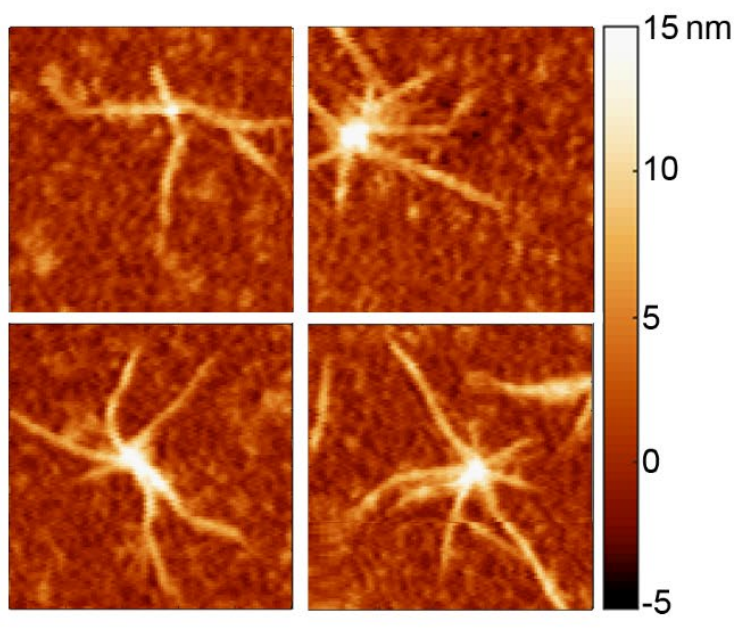

Figure 4.5 POPG vesicles promote a unique htt-exon1(46Q) fibril morphology.

Representative AFM images of a unique fibril morphology formed as a result of htt-exon1(46Q) incubation with POPG lipid vesicles.

While AFM analysis of fibril formation was consistent with ThT assays, AFM also provides information concerning oligomeric aggregates. In the absence of lipid, oligomers of httexon1(46Q) formed within $1 \mathrm{~h}$, and the oligomer population peaked at the $3 \mathrm{~h}$ time point (Fig. 
4.4d). As the experiment progressed, the oligomer population decreased, but not to a statistically significant extent. Morphological analysis of oligomer height and diameter demonstrated that htt oligomers became larger with time in the absence of lipid. After $1 \mathrm{~h}$ of incubation, oligomers were predominately $4-4.5 \mathrm{~nm}$ in height, while the mode size grew to $5.5-6.0 \mathrm{~nm}$ at $8 \mathrm{~h}$ (Fig. 4.4e). In the presence of zwitterionic lipid species (POPC or POPE), oligomer formation again peaked after $3 \mathrm{~h}$ (Fig. 4.4d); however, the population of oligomers at any given time tended to be smaller compared to incubation in the absence of lipid (Fig. 4.4f-g). With POPC lipid vesicles, the population of htt-exon1(46Q) oligomers decreased at each time point relative to control ( $\mathrm{p}<$ 0.05 at 3, 5, and $8 \mathrm{~h}$, Fig. 4.4d). Oligomers formed in the presence of POPC after $1 \mathrm{~h}$ were smaller than control (3-3.5 nm), and these smaller oligomers persisted with time (Fig. 4.4f). After $8 \mathrm{~h}$, htt-exon1(46Q) incubated with POPC formed a more heterogeneous mixture of oligomers with a broad distribution of sizes and a mode height spanning 3-6.5 nm. In the presence of POPE lipid vesicles, the number of oligomers was similar to control through $3 \mathrm{~h}$, but starting with $5 \mathrm{~h}$, the population of oligomers drastically decreased (Fig. 4.4d). Despite the change in the population of oligomers, the morphology of oligomers formed in the presence of POPE was similar to control (Fig. 4.4g). After $1 \mathrm{~h}$, the oligomers had a mode height of 3.5-4.5 nm and they grew to $5.5-6.5 \mathrm{~nm}$ after $8 \mathrm{~h}$.

When incubated with anionic lipids (POPG or POPS), the extent and morphology of oligomers formed was significantly different than in the absence of lipid. With POPG vesicles, the most oligomers were observed at $1 \mathrm{~h}$, and the oligomer population decreased with time (Fig. 4.4d). After $1 \mathrm{~h}$ of incubation, oligomers were slightly smaller than the control with a mode height of 2-2.5 nm (Fig. 4.4h). While oligomers increased in size with time, they remained significantly smaller (3.0-3.5 nm mode height) with POPG after $8 \mathrm{~h}$ of incubation compared to 
control (5.5-6.0 nm mode height). Similar to POPG, the peak oligomer population was observed at $1 \mathrm{~h}$ for htt incubated with POPS vesicles, and the number of oligomers progressively decreased (Fig. 4.4d). Morphological analysis of oligomers formed with POPS vesicles revealed that they were significantly smaller than the control, with a mode height of $2.5-3 \mathrm{~nm}$ after $1 \mathrm{~h}$ of incubation (Fig. 4.4i). At 8 h, oligomer morphology was more heterogeneous but still drastically smaller (3-3.5 nm mode height).

\subsubsection{Htt has varying affinity for different lipid systems}

With the presence of the different lipid systems directly impacting htt aggregation, the extent of htt-exon1(46Q) direct interaction with the different lipid systems was determined by a series of colorimetric membrane binding assays using PDA/lipid vesicles. These vesicles were formed with either POPC, POPE, POPG, or POPS as the lipid component. PDA/lipid vesicles have colorimetric responses (CRs) when exposed to proteins that directly correlate with the extent of interaction. The $\mathrm{CR}$ in the PDA/lipid vesicles is related to transitions of the PDA polymer backbone structure, which is affected by mechanical stress associated with exogenous molecules directly binding and/or inserting into the vesicle. By measurement of the absorbance of both the blue $(640 \mathrm{~nm})$ and red $(500 \mathrm{~nm})$ wavelengths of PDA/lipid vesicles upon exposure to htt-exon1(46Q), the \% CR was obtained, which directly corresponds to the protein-lipid interaction. GST alone does not bind lipids ${ }^{46}$ or result in a CR (data not shown), so here any measured CR is a result of htt-exon1(46Q) interaction with the vesicle. In addition, not cleaving the GST tag inhibits the ability of htt to bind lipids. ${ }^{47}$ As the sensitivity of the CR varies with different lipid systems incorporated into PDA/lipid vesicles, all PDA/lipid vesicle systems were exposed to a large dose of $\mathrm{NaOH}$ to determine the maximal $\% \mathrm{CR}$, and this was used to normalize all data to allow quantitative lipid-to-lipid comparison. 
PDA vesicles formed with POPC as the lipid component exhibited a colorimetric shift in the presence of htt-exon1(46Q) that increased with increasing htt concentration (Fig. 4.6a). Exposure to $5 \mu \mathrm{M}$ htt-exon1(46Q) elicited a CR that grew steadily, reaching a maximum \% CR of $\sim 18 \%$ (Fig. 4.6e). When htt-exon1(46Q) dose was increased to 10 or $20 \mu \mathrm{M}$, the $\%$ CR grew rapidly for the first $3 \mathrm{~h}$, but began to level off with maximum $\% \mathrm{CR}$ of $\sim 35 \%$ and $\sim 45 \%$ for the two htt doses respectively (Fig. 4.6e). POPE/PDA vesicles exhibited a dose dependent increase in CR with increasing dose of htt-exon1(46Q) but to a lesser extent than POPC/PDA vesicles (Fig. 4.6b). In all three cases (5, 10, and $20 \mu \mathrm{M}$ htt-exon1(46Q)), there was limited change in the $\mathrm{CR}$ until $3 \mathrm{~h}$. Then, the \%CR increased for POPE/PDA vesicles exposed to $5 \mu \mathrm{M}$ (maximum $\mathrm{CR} \sim 12 \%$ ), $10 \mu \mathrm{M}$ (maximum $\mathrm{CR} \sim 20 \%$ ), and $20 \mu \mathrm{M}$ (maximum $\mathrm{CR} \sim 25 \%$ ) htt-exon1(46Q)

(Fig. 4.6e).
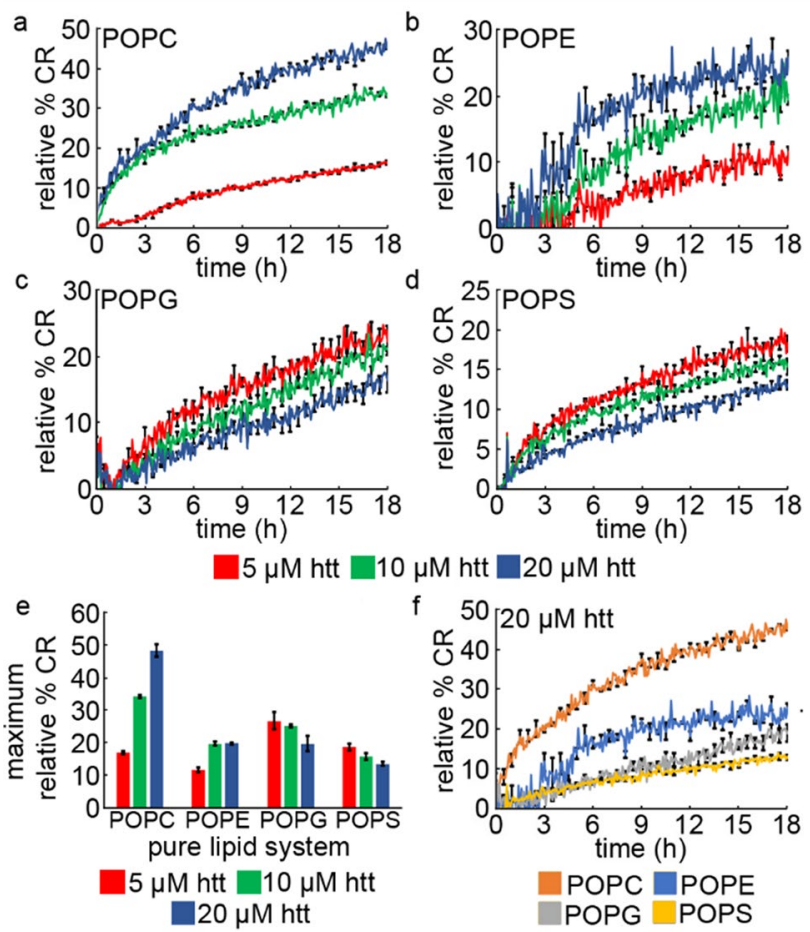

Figure 4.6 Htt-exon1(46Q) membrane association is altered by lipid headgroup. PDA/lipid binding assays for (a) POPC, (b) POPE, (c) POPG, or (d) POPS exposed to 5, 10, or $20 \mu \mathrm{M}$ httexon1(46Q). (e) The maximum \% CR for each condition after $18 \mathrm{~h}$. (f) Direct comparison of the kinetic lipid binding assay for each lipid system when exposed to $20 \mu \mathrm{M}$ htt. Error bars are provided for every sixth data point and represent the SEM. 
A peculiar dose-dependent pattern of htt/lipid interaction was observed by the PDA assays using anionic lipids (Fig. 4.6c-d). While the CR steadily increased when these two lipid systems were exposed to htt, the magnitude of the CR decreased with increasing htt dose (Fig. 4.6e). In the presence of $5 \mu \mathrm{M}$ htt-exon1(46Q), POPG/PDA vesicles exhibited a maximum \% CR of $\sim 27 \%$, while this dropped to $\sim 19 \%$ upon exposure to $20 \mu \mathrm{M}$ htt-exon1(46Q). This trend persisted with POPS/PDA vesicles, as $5 \mu \mathrm{M}$ htt elicited the largest $\% \mathrm{CR}(\sim 20 \%)$ for this system and exposure to $20 \mu \mathrm{M}$ htt only resulted in an $\sim 13 \% \mathrm{CR}$. Due to the decreased interaction of htt with anionic lipids, htt had the largest interaction with anionic lipids at the $5 \mu \mathrm{M}$ dose; however, at the $20 \mu \mathrm{M}$ dose the zwitterionic lipids interacted more strongly with htt (Fig. 4.6e).

\subsubsection{Vesicle charge alters the complexes formed between the lipid binding domain of htt and lipids}

As the ability of htt-exon1 to bind lipids is facilitated by $\mathrm{Nt} 17^{26}$ and it has been shown to act in modifying the aggregation process on membranes ${ }^{22}$, native MS was utilized to determine the propensity and composition of complexes formed between Nt17 and the different lipid systems (POPC, POPE, POPG, and POPS). The interaction mechanism between Nt17 and lipid bilayers does not change upon the addition of glutamines; ${ }^{48}$ therefore, experiments performed with Nt17 can provide insight into the interaction between htt-exon1(46Q) and lipid membranes. Beyond the formation of small multimers, htt aggregation is driven by the polyQ domain ${ }^{49}$ so conclusions about higher-order aggregate formation cannot be drawn from the population of detected Nt17 multimeric species. Nt17 $(20 \mu \mathrm{M})$ was incubated with POPC, POPE, POPG, or POPS lipid vesicles (20:1 lipid:peptide) for $5 \mathrm{~h}$. The $5 \mathrm{~h}$ incubation time was determined from the kinetic PDA data, as htt induces a noticeable colorimetric response in all four lipid systems after $5 \mathrm{~h}$ of incubation. The relative abundance of each species was determined in relation to the 
doubly-charged monomer peptide ion $\left([\mathrm{M}+2 \mathrm{H}]^{2+}\right.$ ion at $\left.m / z \sim 988\right)$. All experiments were performed in triplicate.
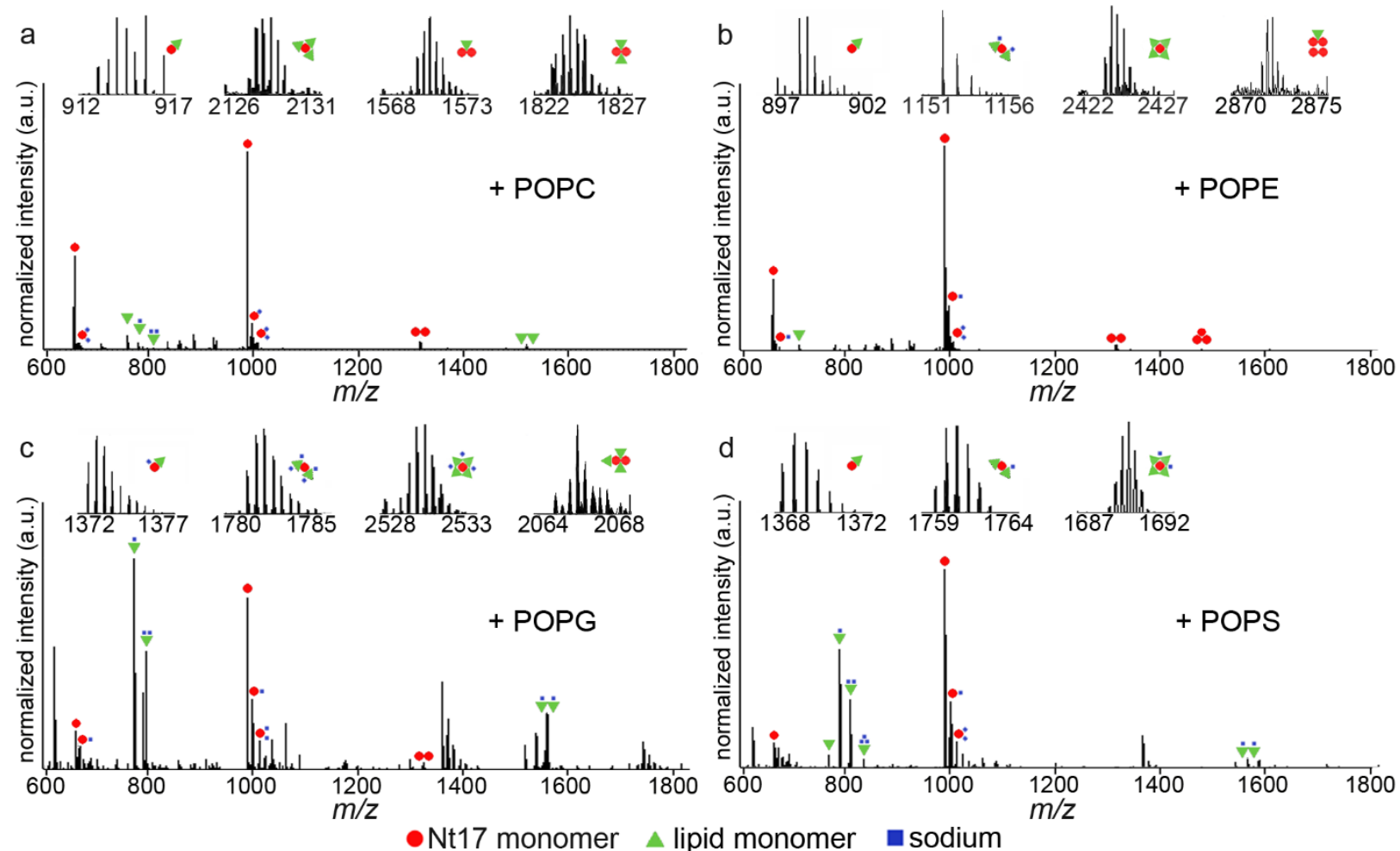

Figure 4.7 Nt17 forms complexes with lipids that are observable by native MS. Mass spectrum showing the ions obtained after ESI of Nt17 with (a) POPC, (b) POPE, (c) POPG, or (d) POPS lipid vesicles after $5 \mathrm{~h}$ of co-incubation. Insets represent expanded regions in which specific observed ions are present.

Ions comprised of peptide-lipid complexes were observed with all four of the lipid systems in various combinations, with the number of peptides per complex ranging from 1-4 and ranging from 1-6 lipids (Fig. 4.7 and Fig. 4.8; Table 4.1). Notably, the composition and relative abundance of the identified ions varied by lipid system (Fig. 4.8). POPC formed the greatest number of distinct complexes ranging with monomeric ( $6 \%$ relative abundance), dimeric $(1.2 \%)$, trimeric $(0.03 \%)$, and tetrameric $(0.009 \%) \mathrm{Nt} 17$ (Figure 4.8, Table 4.1). Twelve distinct ions containing a single Nt17 monomer were observed with POPC, with up to 6 lipid molecules 
associating with $\mathrm{Nt17}$. The diversity of Nt17/POPC species was maintained with dimers (7 ions, up to 5 lipid molecules), trimers (3 ions, up to 5 lipid molecules) and tetramers (4 ions, up to 5 lipid molecules). POPE formed complexes with monomeric (4.5\%), dimeric $(0.13 \%)$, trimeric $(0.02 \%)$, and tetrameric $(0.006 \%) \mathrm{Nt} 17$ as well, but with fewer combinations of peptide and lipid compared with POPC (Figure 4.8, Table 4.1). Only 6 distinct complexes were identified for Nt17 monomers and POPE with up to 4 lipid molecules associated with the peptide. In addition, Nt17/POPE species formed with dimers (2 ions with 3 or 5 lipid molecules), trimers ( 3 ions, up to 4 lipid molecules), and tetramers (2 ions with 1 or 3 lipid molecules).
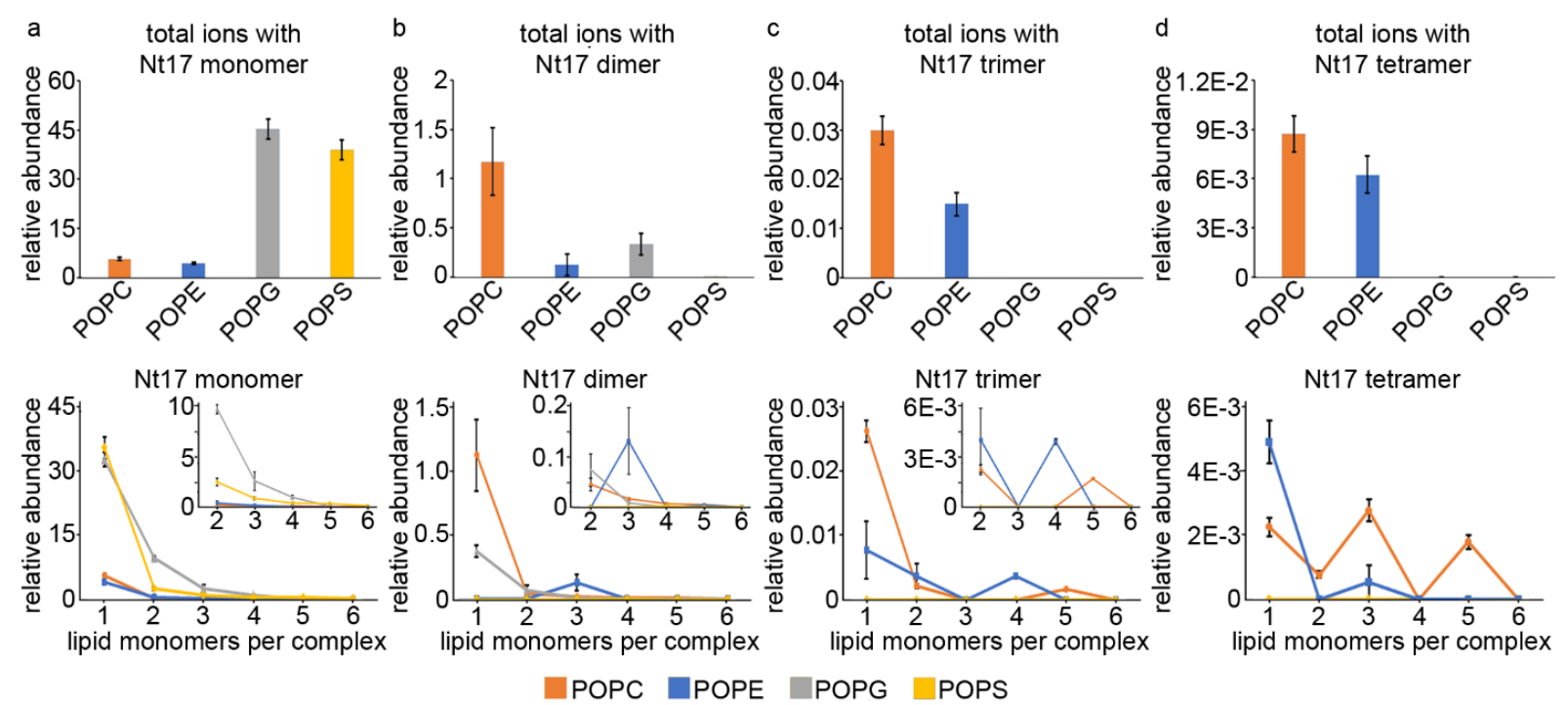

Figure 4.8 Populations of complexes formed with Nt17 and lipid are altered by the lipid headgroup. The relative abundance of ions observed with POPC, POPE, POPG, or POPS lipids and Nt17 (a) monomers, (b) dimers, (c) trimers, or (d) tetramers. The total relative abundance of complexes formed with each $\mathrm{Nt} 17$ species is presented (top), along with the breakdown of relative abundance as a function of lipid content (bottom) for each system. Error bars represent the SEM. 
Table 4.1 Assigned ions for Nt17 incubated with POPC, POPE, POPG, or POPS lipid vesicles along with their mass to charge ratios.

\begin{tabular}{|c|c|c|c|c|c|c|c|c|}
\hline $\operatorname{lon}^{\mathrm{a}}$ & $m / z$ & $\begin{array}{l}\text { Charge } \\
\text { State }\end{array}$ & Ion $^{a}$ & $\mathrm{~m} / \mathrm{z}$ & $\begin{array}{l}\text { Charge } \\
\text { State }\end{array}$ & Ion $^{a}$ & $\mathrm{~m} / \mathrm{z}$ & $\begin{array}{c}\text { Charge } \\
\text { State }\end{array}$ \\
\hline $\mathrm{Nt17}+1 \mathrm{POPC}$ & 2734.63 & 1 & $\mathrm{Nt17}+1 \mathrm{POPE}$ & 1346.79 & 2 & $\mathrm{Nt} 17$ + 1POPG & 2723.61 & 1 \\
\hline $\mathrm{Nt17}+1 \mathrm{POPC}$ & 1367.82 & 2 & Nt17 + 1POPE & 898.19 & 3 & $\mathrm{Nt} 17+\mathrm{POPG}$ & 1362.31 & 2 \\
\hline Nt17 + 1POPC & 912.21 & 3 & Nt17 + 2POPE & 1705.55 & 2 & Nt17 + 1POPG & 908.54 & 3 \\
\hline $\mathrm{Nt17}+2 \mathrm{POPC}$ & 1747.11 & 2 & $\mathrm{Nt} 17+2 \mathrm{POPE}$ & 1137.37 & 3 & $\mathrm{Nt} 17+2 \mathrm{POPG}$ & 1736.59 & 2 \\
\hline $\mathrm{Nt17}+2 \mathrm{POPC}$ & 1165.41 & 3 & Nt17 + 3POPE & 2064.32 & 2 & $\mathrm{Nt} 17+2 \mathrm{POPG}$ & 1158.06 & 3 \\
\hline $\mathrm{Nt17}+3 \mathrm{POPC}$ & 2126.91 & 2 & $\mathrm{Nt} 17+4 \mathrm{POPE}$ & 2423.08 & 2 & $\mathrm{Nt} 17+3 \mathrm{POPG}$ & 2110.88 & 2 \\
\hline $\mathrm{Nt} 17+3 \mathrm{POPC}$ & 1418.60 & 3 & $2 \mathrm{Nt} 17+3 \mathrm{POPE}$ & 3051.34 & 2 & $\mathrm{Nt} 17+3 \mathrm{POPG}$ & 1407.58 & 3 \\
\hline $\mathrm{Nt17}+4 \mathrm{POPC}$ & 2506.70 & 2 & $2 \mathrm{Nt} 17+5 \mathrm{POPE}$ & 3768.87 & 2 & $\mathrm{Nt} 17+4 \mathrm{POPG}$ & 2485.16 & 2 \\
\hline $\mathrm{Nt17}+4 \mathrm{POPC}$ & 1671.80 & 3 & $3 \mathrm{Nt} 17+\mathrm{POPE}$ & 3320.83 & 2 & $\mathrm{Nt} 17$ + 4POPG & 1657.11 & 3 \\
\hline $\mathrm{Nt17}+5 \mathrm{POPC}$ & 2897.99 & 2 & $3 \mathrm{Nt} 17+2 \mathrm{POPE}$ & 2461.05 & 3 & $2 \mathrm{Nt} 17+1 \mathrm{POPG}$ & 2349.33 & 2 \\
\hline $\mathrm{Nt17}+5 \mathrm{POPC}$ & 1925.00 & 3 & $3 \mathrm{Nt} 17+4 \mathrm{POPE}$ & 2931.75 & 3 & $2 \mathrm{Nt} 17+1 \mathrm{POPG}$ & 1566.55 & 3 \\
\hline $\mathrm{Nt} 17+6 \mathrm{POPC}$ & 2180.17 & 3 & $4 \mathrm{Nt} 17+\mathrm{POPE}$ & 2872.23 & 3 & $2 \mathrm{Nt} 17+2 \mathrm{POPG}$ & 1816.07 & 3 \\
\hline $2 \mathrm{Nt} 17+1 \mathrm{POPC}$ & 2354.84 & 2 & $4 \mathrm{Nt} 17+3 \mathrm{POPE}$ & 3350.58 & 3 & & & \\
\hline $2 \mathrm{Nt} 17+1 \mathrm{POPC}$ & 1570.22 & 3 & & & & Nt17 + 1POPS & 1368.27 & 2 \\
\hline $2 \mathrm{Nt} 17+2 \mathrm{POPC}$ & 1823.42 & 3 & & & & $\mathrm{Nt17}+1 \mathrm{POPS}$ & 912.51 & 3 \\
\hline $2 \mathrm{Nt} 17+3 \mathrm{POPC}$ & 3113.93 & 2 & & & & $\mathrm{Nt} 17+2 \mathrm{POPS}$ & 1748.52 & 2 \\
\hline $2 \mathrm{Nt} 17+3 \mathrm{POPC}$ & 2076.62 & 3 & & & & $\mathrm{Nt} 17+2 \mathrm{POPS}$ & 1166.01 & 3 \\
\hline $2 \mathrm{Nt} 17+4 \mathrm{POPC}$ & 2331.13 & 3 & & & & $\mathrm{Nt} 17+3 \mathrm{POPS}$ & 2128.77 & 2 \\
\hline $2 \mathrm{Nt} 17+5 \mathrm{POPC}$ & 2583.01 & 3 & & & & $\mathrm{Nt} 17+3 \mathrm{POPS}$ & 1419.51 & 3 \\
\hline $3 \mathrm{Nt} 17+1 \mathrm{POPC}$ & 2235.90 & 3 & & & & $\mathrm{Nt} 17+4 \mathrm{POPS}$ & 1673.01 & 3 \\
\hline $3 \mathrm{Nt} 17+3 \mathrm{POPC}$ & 2482.21 & 3 & & & & $\mathrm{Nt} 17+5 \mathrm{POPS}$ & 1926.51 & 3 \\
\hline $3 \mathrm{Nt} 17+5 \mathrm{POPC}$ & 3241.02 & 3 & & & & $\mathrm{Nt} 17+6 \mathrm{POPS}$ & 2180.01 & 3 \\
\hline $4 \mathrm{Nt} 17+1 \mathrm{POPC}$ & 2886.25 & 3 & & & & & & \\
\hline $4 \mathrm{Nt} 17+2 \mathrm{POPC}$ & 3139.45 & 3 & & & & & & \\
\hline $4 \mathrm{Nt} 17+3 \mathrm{POPC}$ & 3392.64 & 3 & & & & & & \\
\hline
\end{tabular}


With the anionic lipid systems, fewer distinct complexes were observed, and species with larger peptide content were not detected (Fig. 4.8, Table 4.1). That is, ions containing only a monomer of Nt17 predominated for both POPG (45\%) and POPS (39\%). Three distinct Nt17 dimeric complexes formed with POPG, but these represented a small fraction of Nt17 (0.34\%). No trimeric or tetrameric Nt17 ions were observed with POPG, and multimeric Nt17 ions were completely absent with POPS (Fig. 4.8, Table 4.1). Nt17/POPG formed 9 distinct monomeric complexes with up to 4 lipid molecules, but only 3 dimeric species with up to two lipid molecules were detected (Fig. 4.8, Table 4.1). With POPS, 9 distinct monomeric ions were observed, and Nt17 monomers were associated with up to 6 POPS molecules. Despite the lower number of observed species, POPG and POPS complexes with Nt17 monomers had a significantly larger relative abundance compared with the zwitterionic lipid systems, suggesting that Nt17 monomers may have a higher affinity for anionic lipids.

\subsection{Discussion}

The aggregation process of amyloid proteins is largely dependent on the cellular environment, with one major component being the presence of subcellular membranes comprised of lipids. In this study, the impact of various lipid headgroups on htt-exon1(46Q) aggregation and membrane activity was investigated. The vesicles comprised of anionic lipids significantly enhanced htt-exon1(46Q) fibrillization, even at low lipid:protein ratios; meanwhile, larger lipid:protein ratios were required to observe any impact on aggregation in the presence of zwitterionic lipid vesicles. Anionic charged lipids promoted unique aggregate morphologies, with both fibrils and oligomers exhibiting significantly lower average heights compared with control. The membrane activity of htt-exon1(46Q) as determined by PDA assays was largest for zwitterionic lipid systems in comparison with anionic lipid systems. In addition, complexes 
formed between the Nt17 lipid binding domain htt and anionic lipids were abundant but predominately comprise of a single Nt17 monomer, whereas zwitterionic lipids readily formed complexes with Nt17 multimers. Collectively, these results highlight the complexity of htt-exon1 aggregation and how it is impacted by the presence of lipid membranes.

Understanding the interplay between amyloid proteins and membranous surfaces is critical for understanding a variety of associated toxic mechanisms. Physicochemical features of lipids heavily influence selective interactions of amyloid proteins with membranes ${ }^{50}$ Bilayer fluidity, degree of hydration, ${ }^{51}$ and membrane composition ${ }^{24,25}$ all influence lipid interactions of amyloid proteins. Both htt $\mathrm{t}^{52}$ and $\alpha$-syn ${ }^{53}$ display increased affinity for highly curved lipid membranes, as curvature impacts vesicle lateral pressure and the prevalence of transient packing defects. ${ }^{54}$ Lipid peroxidation, which is known to decrease membrane fluidity and slow lateral diffusion, ${ }^{55}$ decreases the membrane activity of $A \beta{ }^{56}$ Here, the influence of lipid headgroups on physicochemical membrane properties likely underscore the varying membrane activity of htt observed. Nt17 interacts with lipids by forming an amphipathic $\alpha$-helix. ${ }^{57}$ This is a common structural motif found in several proteins that preferentially binds curved membranes due to the high density of transient packing defects induced by curvature that act as putative binding sites. ${ }^{58-60}$ With this in mind, the larger htt membrane affinity for POPE vs POPC (the zwitterionic systems) may be associated with POPE's smaller headgroup, which results in a higher transition temperature and lower lateral fluidity. ${ }^{61}$ The larger lateral mobility of POPC lipids results in a higher density of transient packing defects for Nt17 to bind, increasing the likelihood of htt interaction with the membrane as observed in the PDA assay. When comparing the anionic lipid systems, the higher membrane activity is again observed with the system of higher fluidity (POPG). ${ }^{62}$ In addition, membrane thickness influences membrane affinities of 
other amyloid proteins. $A \beta$ exhibits increased interaction with and disruption of thinner bilayers such as DLPC when compared to thicker bilayers comprised of POPC or DOPC.$^{63}$ In contrast, $\alpha-$ syn has a higher affinity for SUVs comprised of thicker bilayers. ${ }^{64}$ The thickness of bilayers comprised of each lipid system used here has been determined through a combination of experimental and computational approaches: POPC is $39.1 \AA,{ }^{65}$ POPE is $43.2 \AA,{ }^{66}$ POPG is 36.7 $\AA,{ }^{67}$ and POPS is $40.8 \AA$ thick. ${ }^{62}$ In this study, when directly comparing the two zwitterionic lipids or the two anionic lipids, htt exhibited increased interaction with the thinner bilayer (POPC $>$ POPE and POPG $>$ POPS).

Beyond the initial interaction of amyloid proteins with lipid membranes, these surfaces can promote alternative aggregation mechanisms, resulting in unique aggregate morphologies and stabilization of intermediates structures. ${ }^{50}$ This appears to be highly dependent on the composition of the lipid membrane. ${ }^{23,24,68,69}$ POPC/POPS membranes promoted a distinct Nt17mediated htt aggregation mechanism that catalyzed fibrillization via membrane anchoring and two-dimensional diffusion. ${ }^{22}$ In contrast, total brain lipid extracts stabilize oligomers of a fragmented polyQ peptide containing $\mathrm{Nt} 17^{26}$ and impede fibrillization of htt-exon1. Here, four different lipid systems influence the rate of htt-exon1(46Q) aggregation to varying extents, with modifying effects on aggregate morphology (both fibrils and oligomers). Zwitterionic lipid systems required a large lipid to protein ratio to impact the rate of fibril formation with POPC enhancing aggregation and POPE repressing it. Even so, the resulting aggregate morphologies were similar to control. Anionic lipids induced the most significant alterations to htt-exon1(46Q) aggregation, similar to what has been observed with other amyloid proteins. For example, hIAPP aggregation is altered by acidic phospholipids, resulting in the formation of off-pathway oligomers. ${ }^{68}$ Interestingly, hIAPP also interacts with lipids via an amphipathic $\alpha$-helical domain. 
In the case of $\alpha$-syn, acidic phospholipid membranes enhance fibrillization. ${ }^{53}$ With httexon1(46Q), fibrils and oligomers formed in the presence of anionic phospholipids displayed unique morphological features, i.e. smaller initial oligomers and thinner fibrils, suggesting an alternative aggregation mechanism. Fibril polymorphisms of htt-exon1 results from the placement of flanking domains rather than changes in the polyglutamine core packing ${ }^{70}$ and in this case may be a function of the strong association mechanism between Nt17 monomers and anionic lipids. This would be consistent with the presence of POPS in the model membranes used in the study by Pandey et al. that suggested a unique Nt17 mediated aggregation mechanism. ${ }^{22}$

As alluded to above, the effect of lipids on htt-exon1(46Q) aggregation is likely mediated through the direct interaction with Nt17. Based on computational studies, the interaction of Nt17 with phospholipid bilayers involves four basic steps: approach, reorganization, anchoring, and insertion. ${ }^{48}$ Despite sharing the same basic steps, the precise mechanism of Nt17 binding is influenced by membrane composition and properties. ${ }^{45}$ Here, it appears lipid headgroup charge alters the progression through this $\mathrm{Nt} 17$ membrane binding process. When examining the interaction of anionic lipid systems with htt, a high relative abundance of ions including Nt17 monomer were observed by native MS, but the colorimetric response in the PDA assay was low for both systems. Combined, these results indicate an inefficiency in the binding process between the anchoring and insertion step that can result in the formation of monomeric species detectable by MS without invoking a large PDA colorimetric response. Despite inefficient insertion indicated by the low PDA response, localization of htt monomers at the membrane surface would be enhanced by electrostatic interaction between the net positively charged Nt17 and anionic lipid headgroups. ${ }^{71}$ This electrostatic interaction could also lend some stability to any ions 
formed. This localization at the anionic lipid surface results in a region of high htt concentration, enhancing aggregation as observed by ThT and AFM. Such a scenario is consistent with the observation by ThT that increasing the lipid to protein ratio reduces the observed enhancement of htt aggregation as the available htt would be spread across a larger total vesicle surface area, decreasing the localized concentration.

In $\mathrm{HD}$, one potential toxic mechanisms is that htt aggregation disrupts the membrane integrity of specific organelles. ${ }^{29,33,35}$ Mutant htt demonstrates enhanced binding of lipid membranes and disrupts phospholipid bilayer structural integrity in a polyQ-length dependent manner. ${ }^{30-32} \mathrm{Htt}$ aggregates accumulate brain lipids ${ }^{27,37}$ and membranous structures are incorporated into htt inclusions. ${ }^{38,39}$ The factors determining the affinity of htt for specific membranous organelles are not completely understood, but results presented here indicate a role for membrane composition. Htt is associated with mitochondria ${ }^{29,35}$ and endoplasmic reticulum $(E R)^{33}$ membranes in vitro, each of which contain a large percentage of the phosphatidylcholine (PC) lipids (46 and 54\%, respectively ${ }^{72}$ ), for which htt-exon1(46Q) demonstrated the largest membrane activity. Specifically, htt associates with the outer mitochondrial membrane, which has a higher PC lipid content than the inner membrane. ${ }^{73}$ In addition, htt-exon1(46Q) monomers had a high affinity for the anionic lipid headgroups, which correlates with the association of htt to acidic phospholipids at the plasma membrane. ${ }^{30}$ The effect of anionic lipids on htt membrane activity and aggregation decreased with increasing lipid:protein ratios. This is consistent with the observation that the affinity of htt for the phosphatidylserine of plasma membranes decreases with increasing lysate concentration. ${ }^{30}$ In addition to the studies reported here, other membrane components such as cholesterol, sphingomyelin, and GM1 all influence the membrane activity of htt. ${ }^{24,25}$ 
In addition to potentially toxic mechanisms, localization and normal functions of htt can be associated with its affinity for lipid membranes. Htt is directly involved in ER-to-Golgi transport and vesicle fusion at the plasma membrane, making it a crucial player in secretory pathways. ${ }^{36}$ Variability in membrane affinity results in selective localization of htt to specific subcellular components, such as the nucleus, ${ }^{74}$ as well as trafficking to autophagic vacuoles, mitochondria, ER, and Golgi. ${ }^{75,76}$ The importance of Nt17 in these functions is supported by abundant sites of post-translational modifications, such as phosphorylation, that result in changes to htt trafficking and nuclear export. ${ }^{77-79}$ With the importance of Nt17 affinity for specific lipids in htt localization and trafficking, alterations to membrane composition that are associated with HD may play a role in toxic disruption of normal htt function. ${ }^{28,80,81}$

\subsection{References}

1. Xue, W.-F., Homans, S. W. \& Radford, S. E. Systematic analysis of nucleation-dependent polymerization reveals new insights into the mechanism of amyloid self-assembly. Proc. Natl. Acad. Sci. U.S.A 105, 8926-8931 (2008).

2. Chiti, F. \& Dobson, C. M. Protein Misfolding, Functional Amyloid, and Human Disease. Annu. Rev. of Biochem. 75, 333-366 (2006).

3. Kar, K., Jayaraman, M., Sahoo, B., Kodali, R. \& Wetzel, R. Critical nucleus size for diseaserelated polyglutamine aggregation is repeat length dependent. Nat. Struct. Mol. Biol. 18, 328-336 (2011).

4. Arndt, J. R. et al. Huntingtin N-terminal monomeric and multimeric structures destabilized by covalent modification of heteroatomic residues. Biochemistry 54, 4285-4296 (2015).

5. Burke, K. A., Godbey, J. \& Legleiter, J. Assessing mutant huntingtin fragment and polyglutamine aggregation by atomic force microscopy. Methods 53, 275-284 (2011).

6. Counterman, A. E., Hilderbrand, A. E., Barnes, C. A. S. \& Clemmer, D. E. Formation of peptide aggregates during ESI: Size, charge, composition, and contributions to noise. J. Am. Soc. Spectrom. 12, 1020-1035 (2001).

7. Davies, S. W. et al. Formation of neuronal intranuclear inclusions underlies the neurological dysfunction in mice transgenic for the HD mutation. Cell 90, 537-548 (1997). 
8. Gutekunst, C. A. et al. Nuclear and neuropil aggregates in Huntington's disease: relationship to neuropathology. J. Neurosci. 19, 2522-2534 (1999).

9. Saudou, F., Finkbeiner, S., Devys, D. \& Greenberg, M. E. Huntingtin acts in the nucleus to induce apoptosis but death does not correlate with the formation of intranuclear inclusions. Cell 95, 55-66 (1998).

10. Arrasate, M., Mitra, S., Schweitzer, E. S., Segal, M. R. \& Finkbeiner, S. Inclusion body formation reduces levels of mutant huntingtin and the risk of neuronal death. Nature 431, 805-810 (2004).

11. Olshina, M. A. et al. Tracking mutant huntingtin aggregation kinetics in cells reveals three major populations that include an invariant oligomer pool. J. Biol. Chem. 285, 21807-21816 (2010).

12. Lajoie, P. \& Snapp, E. L. Formation and toxicity of soluble polyglutamine oligomers in living cells. PLoS ONE 5, e15245 (2010).

13. Legleiter, J. et al. Mutant Huntingtin Fragments Form Oligomers in a Polyglutamine Lengthdependent Manner in Vitro and in Vivo. J. Biol. Chem. 285, 14777-14790 (2010).

14. Sahl, S. J., Weiss, L. E., Duim, W. C., Frydman, J. \& Moerner, W. E. Cellular Inclusion Bodies of Mutant Huntingtin Exon 1 Obscure Small Fibrillar Aggregate Species. Sci. Rep. 2, 1-7 (2012).

15. Sahl, S. J. et al. Delayed emergence of subdiffraction-sized mutant huntingtin fibrils following inclusion body formation. Q. Rev. Biophys. 49, e2 (2016).

16. Lu, M. et al. Live-cell super-resolution microscopy reveals a primary role for diffusion in polyglutamine-driven aggresome assembly. J. Biol. Chem. 294, 257-268 (2019).

17. Nucifora, L. G. et al. Identification of novel potentially toxic oligomers formed in vitro from mammalian-derived expanded huntingtin exon-1 protein. J. Biol. Chem. 287, 16017-16028 (2012).

18. Kim, Y. E. et al. Soluble Oligomers of PolyQ-Expanded Huntingtin Target a Multiplicity of Key Cellular Factors. Mol. Cell 63, 951-964 (2016).

19. Drombosky, K. W. et al. Mutational analysis implicates the amyloid fibril as the toxic entity in Huntington's disease. Neurobiol. Dis. 120, 126-138 (2018).

20. Pieri, L., Madiona, K., Bousset, L. \& Melki, R. Fibrillar $\alpha$-Synuclein and Huntingtin Exon 1 Assemblies Are Toxic to the Cells. Biophys. J. 102, 2894-2905 (2012).

21. Bäuerlein, F. J. B. et al. In Situ Architecture and Cellular Interactions of PolyQ Inclusions. Cell 171, 179-187.e10 (2017). 
22. Pandey, N. K. et al. The 17-residue-long N terminus in huntingtin controls stepwise aggregation in solution and on membranes via different mechanisms. J. Biol. Chem. 293, 2597-2605 (2018).

23. Beasley, M. et al. Lipid membranes influence the ability of small molecules to inhibit huntingtin fibrillization. Biochemistry (2019) doi:10.1021/acs.biochem.9b00739.

24. Gao, X. et al. Cholesterol Modifies Huntingtin Binding to, Disruption of, and Aggregation on Lipid Membranes. Biochemistry 55, 92-102 (2016).

25. Chaibva, M. et al. Sphingomyelin and GM1 Influence Huntingtin Binding to, Disruption of, and Aggregation on Lipid Membranes. ACS Omega 3, 273-285 (2018).

26. Burke, K. A., Kauffman, K. J., Umbaugh, C. S., Frey, S. L. \& Legleiter, J. The Interaction of Polyglutamine Peptides with Lipid Membranes Is Regulated by Flanking Sequences Associated with Huntingtin. J. Biol. Chem. 288, 14993-15005 (2013).

27. Suopanki, J. et al. Interaction of huntingtin fragments with brain membranes - clues to early dysfunction in Huntington's disease. J. Neurochem. 96, 870-884 (2006).

28. Eckmann, J. et al. Mitochondrial Membrane Fluidity is Consistently Increased in Different Models of Huntington Disease: Restorative Effects of Olesoxime. Mol. Neurobiol. 50, 107118 (2014).

29. Choo, Y. S., Johnson, G. V. W., MacDonald, M., Detloff, P. J. \& Lesort, M. Mutant huntingtin directly increases susceptibility of mitochondria to the calcium-induced permeability transition and cytochrome c release. Hum. Mol. Genet. 13, 1407-1420 (2004).

30. Kegel, K. B. et al. Huntingtin Associates with Acidic Phospholipids at the Plasma Membrane. J. Biol. Chem. 280, 36464-36473 (2005).

31. B, K.-G. K. Huntingtin Interactions with Membrane Phospholipids: Strategic Targets for Therapeutic Intervention? J. Huntingtons Dis. 239-250 (2013) doi:10.3233/JHD-130068.

32. Lee, W.-C. M., Yoshihara, M. \& Littleton, J. T. Cytoplasmic aggregates trap polyglutaminecontaining proteins and block axonal transport in a Drosophila model of Huntington's disease. Proc. Natl. Acad. Sci. U.S.A. 101, 3224-3229 (2004).

33. Guedes-Dias, P. \& Holzbaur, E. L. F. Huntingtin Fibrils Poke Membranes. Cell 171, 32-33 (2017).

34. Chang, D. T. W., Rintoul, G. L., Pandipati, S. \& Reynolds, I. J. Mutant huntingtin aggregates impair mitochondrial movement and trafficking in cortical neurons. Neurobiol. Dis. 22, 388400 (2006).

35. Orr, A. L. et al. N-Terminal Mutant Huntingtin Associates with Mitochondria and Impairs Mitochondrial Trafficking. J. Neurosci. 28, 2783-2792 (2008). 
36. Brandstaetter, H., Kruppa, A. J. \& Buss, F. Huntingtin is required for ER-to-Golgi transport and for secretory vesicle fusion at the plasma membrane. Dis. Model Mech. 7, 1335-1340 (2014).

37. Valencia, A. et al. Mutant huntingtin and glycogen synthase kinase 3-beta accumulate in neuronal lipid rafts of a presymptomatic knock-in mouse model of Huntington's disease. $J$. Neurosci. Res. 88, 179-190 (2010).

38. Qin, Z.-H. et al. Huntingtin bodies sequester vesicle-associated proteins by a polyprolinedependent interaction. J. Neurosci. 24, 269-281 (2004).

39. Kegel, K. B. et al. Huntingtin expression stimulates endosomal-lysosomal activity, endosome tubulation, and autophagy. J. Neurosci. 20, 7268-7278 (2000).

40. Shirendeb, U. P. et al. Mutant huntingtin's interaction with mitochondrial protein Drp1 impairs mitochondrial biogenesis and causes defective axonal transport and synaptic degeneration in Huntington's disease. Hum. Mol. Genet. 21, 406-420 (2012).

41. Ruan, Q., Lesort, M., MacDonald, M. E. \& Johnson, G. V. W. Striatal cells from mutant huntingtin knock-in mice are selectively vulnerable to mitochondrial complex II inhibitorinduced cell death through a non-apoptotic pathway. Hum. Mol. Genet. 13, 669-681 (2004).

42. Thompson, L. M. et al. IKK phosphorylates Huntingtin and targets it for degradation by the proteasome and lysosome. J. Cell Biol. 187, 1083-1099 (2009).

43. Burke, K. A. \& Legleiter, J. Atomic Force Microscopy Assays for Evaluating Polyglutamine Aggregation in Solution and on Surfaces. in Tandem Repeats in Genes, Proteins, and Disease: Methods and Protocols (eds. Hatters, D. M. \& Hannan, A. J.) 21-40 (Humana Press, 2013). doi:10.1007/978-1-62703-438-8_2.

44. van den Heuvel, R. H. H. \& Heck, A. J. R. Native protein mass spectrometry: from intact oligomers to functional machineries. Curr. Opin. Chem. Biol. 8, 519-526 (2004).

45. Karanji, A. K. et al. Investigating the interactions of the first 17 amino acid residues of Huntingtin with lipid vesicles using mass spectrometry and molecular dynamics. J. Mass Spectrom. 55, e4470 (2020).

46. Han, B.-K. \& Emr, S. D. Phosphoinositide [PI(3,5)P2] lipid-dependent regulation of the general transcriptional regulator Tup1. Genes Dev. 25 984-995 (2011)

47. Burke, K. A., Hensal, K. M., Umbaugh, C. S., Chaibva, M. \& Legleiter, J. Huntingtin disrupts lipid bilayers in a polyQ-length dependent manner. Biochim. Biophys. Acta Biomembr. 1828, 1953-1961 (2013).

48. Côté, S., Wei, G. \& Mousseau, N. Atomistic mechanisms of huntingtin N-terminal fragment insertion on a phospholipid bilayer revealed by molecular dynamics simulations. Proteins 82, 1409-1427 (2014). 
49. Li, S.-H. \& Li, X.-J. Aggregation of N-Terminal Huntingtin is Dependent on the Length of Its Glutamine Repeats. Hum. Mol. Genet. 7, 777-782 (1998).

50. Burke, K. A., Yates, E. A. \& Legleiter, J. Biophysical Insights into How Surfaces, Including Lipid Membranes, Modulate Protein Aggregation Related to Neurodegeneration. Front. Neurol. 4, (2013).

51. Gorbenko, G. P. \& Kinnunen, P. K. J. The role of lipid-protein interactions in amyloid-type protein fibril formation. Chem. Phys. Lipids 141, 72-82 (2006).

52. Chaibva, M., Burke, K. A. \& Legleiter, J. Curvature Enhances Binding and Aggregation of Huntingtin at Lipid Membranes. Biochemistry 53, 2355-2365 (2014).

53. Iyer, A. \& Claessens, M. M. A. E. Disruptive membrane interactions of alpha-synuclein aggregates. Biochim. Biophys. Acta, Proteins Proteomics 1867, 468-482 (2019).

54. Davidson, W. S., Jonas, A., Clayton, D. F. \& George, J. M. Stabilization of $\alpha$-Synuclein Secondary Structure upon Binding to Synthetic Membranes. J. Biol. Chem. 273, 9443-9449 (1998).

55. Borst, J. W., Visser, N. V., Kouptsova, O. \& Visser, A. J. W. G. Oxidation of unsaturated phospholipids in membrane bilayer mixtures is accompanied by membrane fluidity changes. Biochim. Biophys. Acta, Mol. Cell Biol. Lipids 1487, 61-73 (2000).

56. Pilkington, A. W. et al. Hydrogen Peroxide Modifies A $\beta-M e m b r a n e$ Interactions with Implications for A $\beta 40$ Aggregation. Biochemistry 58, 2893-2905 (2019).

57. Michalek, M., Salnikov, E. S. \& Bechinger, B. Structure and Topology of the Huntingtin 117 Membrane Anchor by a Combined Solution and Solid-State NMR Approach. Biophys. J. 105, 699-710 (2013).

58. Drin, G. \& Antonny, B. Amphipathic helices and membrane curvature. FEBS Lett. 584, 1840-1847 (2010).

59. Drin, G. et al. A general amphipathic alpha-helical motif for sensing membrane curvature. Nat. Struct. Mol. Biol. 14, 138-146 (2007).

60. Hatzakis, N. S. et al. How curved membranes recruit amphipathic helices and protein anchoring motifs. Nat. Chem. Biol. 5, 835-841 (2009).

61. Phase Transition Temperatures for Glycerophospholipids. Avanti Polar Lipids https://avantilipids.com/tech-support/physical-properties/phase-transition-temps.

62. Mukherjee, S. et al. Accelerated Molecular Dynamics Simulation Analysis of MSI-594 in a Lipid Bilayer. Phys. Chem. Chem. Phys. 19, 19289-19299 (2017).

63. Korshavn, K. J. et al. Reduced Lipid Bilayer Thickness Regulates the Aggregation and Cytotoxicity of Amyloid- $\beta$. J. Biol. Chem. 292, 4638-4650 (2017). 
64. Wang, G.-F., Li, C. \& Pielak, G. J. 19F NMR studies of $\alpha$-synuclein-membrane interactions. Protein Sci. 19, 1686-1691 (2010).

65. Kučerka, N., Nieh, M.-P. \& Katsaras, J. Fluid phase lipid areas and bilayer thicknesses of commonly used phosphatidylcholines as a function of temperature. Biochim. Biophys. Acta, Biomembr. 1808, 2761-2771 (2011).

66. Leekumjorn, S. \& Sum, A. K. Molecular Characterization of Gel and Liquid-Crystalline Structures of Fully Hydrated POPC and POPE Bilayers. J. Phys. Chem. B 111, 6026-6033 (2007).

67. Kučerka, N., Holland, B. W., Gray, C. G., Tomberli, B. \& Katsaras, J. Scattering Density Profile Model of POPG Bilayers As Determined by Molecular Dynamics Simulations and Small-Angle Neutron and X-ray Scattering Experiments. J. Phys. Chem. B 116, 232-239 (2012).

68. Zhang, J., Tan, J., Pei, R. \& Ye, S. Acidic Environment Significantly Alters Aggregation Pathway of Human Islet Amyloid Polypeptide at Negative Lipid Membrane. Langmuir 36, 1530-1537 (2020).

69. Hane, F., Drolle, E., Gaikwad, R., Faught, E. \& Leonenko, Z. Amyloid- $\beta$ aggregation on model lipid membranes: an atomic force microscopy study. J. Alzheimers Dis. 26, 485-494 (2011).

70. Lin, H.-K. et al. Fibril polymorphism affects immobilized non-amyloid flanking domains of huntingtin exon 1 rather than its polyglutamine core. Nat. Commun. 8, 1-12 (2017).

71. Tao, M., Pandey, N. K., Barnes, R., Han, S. \& Langen, R. Structure of Membrane-Bound Huntingtin Exon 1 Reveals Membrane Interaction and Aggregation Mechanisms. Structure 27, 1570-1580.e4 (2019).

72. C2: Lipid Distribution in Cells. Biology LibreTexts https://bio.libretexts.org/Bookshelves/Biochemistry/Book\%3A_Biochemistry_Online_(Jaku bowski) $/ 01 \% 3 \mathrm{~A}$ LIPID_STRUCTURE/1.3\%3A_Dynamics_of_Membrane_Lipids/C $\overline{2} \% 3 \mathrm{~A}$ Lipid_Distribution_in_Cells (2016).

73. Comte, J., Maǐsterrena, B. \& Gautheron, D. C. Lipid composition and protein profiles of outer and inner membranes from pig heart mitochondria. Comparison with microsomes. Biochim. Biophys. Acta, Biomembr. 419, 271-284 (1976).

74. Hughes, A. \& Jones, L. Huntingtin localisation studies - a technical review. PLoS Curr. 3, (2011).

75. Atwal, R. S. et al. Huntingtin has a membrane association signal that can modulate huntingtin aggregation, nuclear entry and toxicity. Hum. Mol. Genet. 16, 2600-2615 (2007). 
76. Rockabrand, E. et al. The first 17 amino acids of Huntingtin modulate its sub-cellular localization, aggregation and effects on calcium homeostasis. Hum. Mol. Genet. 16, 61-77 (2007).

77. Aiken, C. T. et al. Phosphorylation of threonine 3: implications for Huntingtin aggregation and neurotoxicity. J. Biol. Chem. 284, 29427-29436 (2009).

78. X, G. et al. Serines 13 and 16 are critical determinants of full-length human mutant huntingtin induced disease pathogenesis in HD mice. Neuron 64, (00:00:00.0).

79. Zheng, Z., Li, A., Holmes, B. B., Marasa, J. C. \& Diamond, M. I. An N-terminal Nuclear Export Signal Regulates Trafficking and Aggregation of Huntingtin (Htt) Protein Exon 1. J. Biol. Chem. 288, 6063-6071 (2013).

80. Block, R. C., Dorsey, E. R., Beck, C. A., Brenna, J. T. \& Shoulson, I. Altered Cholesterol and Fatty Acid Metabolism in Huntington Disease. J. Clin. Lipidol. 4, 17-23 (2010).

81. Karasinska, J. M. \& Hayden, M. R. Cholesterol metabolism in Huntington disease. Nat. Rev. Neurol. 7, 561-572 (2011). 


\section{Physicochemical Properties Altered by the Tail Group of Lipid Membranes Influence Huntingtin Aggregation and Lipid Binding}

Huntington's Disease is a neurodegenerative disorder caused by an expanded polyglutamine (polyQ) domain within the huntingtin protein (htt) that spurs the formation of toxic protein aggregates. Htt directly interact with membranes, which influence the aggregation process and can lead to membrane abnormalities. These interactions are facilitated by the $17 \mathrm{~N}$ terminal residues (Nt17) which form an amphipathic $\alpha$-helix $(\mathrm{AH})$ implicated in both lipid binding and aggregation. Here, the impact of lipid tail unsaturation (DMPC, POPC, or DOPC) on htt/lipid interactions was investigated. The influence of the lipid vesicles on aggregation did not correlate with the degree of htt-membrane association, indicating that membrane properties alter htt aggregation. This can be linked to the initial lipid-binding mechanisms that influence Nt17 orientation on the membrane surface based on compatibility between specific hydrophobic residue and membrane defects.

\subsection{Introduction}

Numerous neurodegenerative diseases are characterized by formation and deposition of toxic protein aggregates in tissues. Huntington's Disease (HD) is caused by an expanded polyglutamine (polyQ) domain within the huntingtin protein (htt) that promotes aggregation. PolyQ expansion beyond a threshold of $\sim 35$ residues is associated with disease and correlates with the extent of aggregation, age of onset, and severity of symptoms. ${ }^{1-3} \mathrm{Htt}$ aggregation is a complex, multipathway process that results in a heterogeneous mixture of aggregates ranging from oligomers and annular aggregates ${ }^{4,5}$ to $\beta$-sheet-rich fibrils and inclusion bodies. ${ }^{6-11}$ The heterogeneity of htt aggregation complicates assigning specific modes of toxicity to discreet 
aggregate species. While aggregation leads to the formation of toxic aggregates, other species may prove benign or even protective. The diffuse fraction of htt within cells appears particularly toxic, ${ }^{11,12}$ however, this fraction is populated by a complex mixture of monomers, oligomers, amorphous aggregates, and fibrils. ${ }^{13-17}$ Despite this complexity, some aggregate species are linked to specific toxic mechanisms. Htt oligomers, ${ }^{12,14,18}$ fibrils, ${ }^{19,20}$ and even inclusions ${ }^{21}$ have all been linked to a variety of toxic mechanisms.

The polyQ domain of htt is flanked on the N-terminal end by 17 amino acids (Nt17) that act as a lipid binding domain and govern the interaction of htt with membranes. ${ }^{22-24} \mathrm{Nt17}$ is intrinsically disordered in bulk solution, but undergoes a structural transition to an amphipathic $\alpha$-helix (AH) in the presence of binding partners like lipid membranes. ${ }^{23-25}$ The interaction between htt and phospholipid membranes via Nt17 plays a role in a number of normal htt functions ${ }^{26,27}$ and cytotoxic mechanisms. ${ }^{26,28} \mathrm{Nt} 17$ functions in trafficking htt to mitochondria, vesicles, the ER, and the nucleus ${ }^{15-17}$ however, these membrane interactions can become detrimental with mutant htt. Mutant htt damages and destabilizes membranes, ${ }^{29,30}$ and this correlates with polyQ length. ${ }^{31}$ This may result from the aggregation process, as htt aggregates incorporate membranous assemblies ${ }^{32,33}$ and brain lipids ${ }^{30,34}$ into their structures. These interaction between htt aggregates and membranes are linked to cell death, ${ }^{35}$ synaptic degeneration, ${ }^{36}$ disruption of htt trafficking, ${ }^{37}$ and deformation of membranous organelles. ${ }^{38-40}$

Nt17 not only facilitates htt's interaction with phospholipids but also forms intermolecular associations that promote htt aggregation. ${ }^{41-44}$ This interaction results in $\alpha$-helical oligomers that act as nucleation sites for fibrillization. ${ }^{44}$ Potentially due to the dual role of Nt17 in lipid binding and aggregation, membranes alter the kinetics and mechanism of htt aggregation. ${ }^{30,41} \mathrm{POPC} / \mathrm{PS}$ vesicles catalyze htt aggregation through a unique Nt17-controlled 
membrane-mediated mechanism. ${ }^{41}$ On the other hand, brain lipid extracts inhibit htt fibrillization ${ }^{45}$ and stabilize specific oligomer species. ${ }^{46}$ Increased cholesterol ${ }^{47}$ sphingomyelin, and $\mathrm{GM} 1{ }^{46}$ content in membranes also influence the morphology of htt aggregates.

The conformational rearrangement of $\mathrm{Nt} 17$ from intrinsically disordered to $\mathrm{AH}$ is a structural transition also found in other proteins that play a role in membrane-associated cellular processes such as intracellular membrane targeting ${ }^{48}$ curvature sensing,${ }^{49}$ and membrane remodeling. ${ }^{50}$ Many of these proteins function in the early secretory pathway and nuclear envelope ${ }^{49,51}$ while others function in the distinctly different lipid environment of the endosomal or plasma membranes. ${ }^{52}$ The interaction between the $\mathrm{AH}$ and specific membranes are crucial for proper function, but the properties contributing to their membrane affinity are not well understood. Certain factors that influence the membrane selectivity of AH-containing proteins like Golgi tethers or $\alpha$-synuclein have been identified, but they rely on specific chemical properties of the helix such as a lack of charged polar residues or a hydrophobic face consisting entirely of small residues. ${ }^{52}$ These sequences result in an unbalanced helix, where either the electrostatic or hydrophobic effects dictate the mechanism of AH binding. So far, few mechanistic details have been identified for the association of AHs, such as Nt17, that contain a well-defined hydrophobic face as well as charged polar residues.

Understanding the physicochemical properties of membranes that influence Nt17's interaction with lipids represents an intriguing target for the reduction of htt aggregation, lipid binding, and membrane disturbance and can deepen the understanding of $\mathrm{AH}$ membrane binding mechanisms. To determine how membrane physicochemical properties influence the behavior of htt, the extent of htt aggregation and membrane association was investigated in the presence of three pure lipid systems that have the same headgroup, but different tails (DMPC, POPC, and 
DOPC). First, the impact of the lipid vesicles on the kinetics of htt aggregate formation and the morphology of aggregates was elucidated with Thioflavin-T aggregation (ThT) assays and atomic force microscopy (AFM). In addition, the degree of htt membrane association and complex formation with each lipid system was determined via polydiacetylene (PDA) lipid binding assays and native mass spectrometry (MS). Finally, the initial interaction mechanism of Nt17 with each lipid bilayer was determined via molecular dynamics (MD) simulations. Our studies show that each lipid's effect on the extent of htt aggregate formation does not correlate with the degree of htt-lipid interaction, indicating that the mechanism of membrane-mediated aggregation is altered uniquely by each lipid system. Also, the mechanism of Nt17 interaction with the membranes is driven by a combination of peptide and membrane properties; the size of both the peptide hydrophobic amino acids and the membrane defects determine the approach and partitioning of Nt17 into the bilayers.

\subsection{Methods}

\subsubsection{Purification of glutathione S-transferase (GST)-htt exon 1 fusion protein}

Htt-exon1 with 46 repeat glutamine residues (htt-exon1(46Q)) was expressed in E. coli as a glutathione S-transferase (GST) fusion protein and purified as previously described. ${ }^{53}$ Briefly, the fusion proteins were purified via affinity liquid chromatography using a GST column (BioRad LPLC). Relevant fractions were tested by SDS-PAGE to assess purity. Fusion protein solutions were dialyzed for two days to remove glutathione that was used to elute fusion proteins from the column. To remove pre-existing aggregates, fusion proteins were subjected to high speed centrifugation $(22000 \times \mathrm{g})$ prior to any experiment. Addition of Factor Xa (Promega, Madison, WI) cleaved the GST tag and initiated aggregation. 


\subsubsection{Lipid vesicle formation}

1,2-dimyristoyl-sn-glycero-3-phosphocholine (DMPC), 1-palmitoyl-2-oleoyl-glycero-3phosphocholine (POPC), and 1-palmitoyl-2-oleoyl-glycero-3-phosphocholine (DOPC) were obtained from Avanti Polar Lipids as dried films and rehydrated in tris buffer $(\mathrm{pH}=7.4)$ for $1 \mathrm{~h}$ at $1 \mathrm{mg} / \mathrm{mL}$. Lipid solution were subjected to ten freeze-thaw cycles, proceeded by bath sonicated for $1 \mathrm{~h}$.

\subsubsection{Thioflavin $T$ assay}

To monitor fibril formation as a function of time, a Thioflavin T (ThT, Sigma-Aldrich, St. Louis, MO) aggregation assay was performed. Htt-exon1(46Q) $(20 \mu \mathrm{M})$ was incubated with ThT $(125 \mu \mathrm{M})$ in the presence and absence of various lipid vesicles. In the lipid conditions, lipid concentration was $100 \mu \mathrm{M}, 200 \mu \mathrm{M}$, or $400 \mu \mathrm{M}$ resulting in 5:1, 10:1, or 20:1 lipid:protein ratios. Reactions were run in black Costar 96-well plates with clear flat bottoms, and ThT fluorescence was monitored using a SpectraMax M2 microplate reader. Experiments were run at $37{ }^{\circ} \mathrm{C}$ with $440 \mathrm{~nm}$ excitation and $484 \mathrm{~nm}$ emission, with readings every $5 \mathrm{~min}$ for $18 \mathrm{~h}$. Relative maximum fluorescence for each condition was determined by finding the maximum fluorescence intensity and normalizing to the maximum fluorescence of the huntingtin control $(100 \%)$. The relative rate for each condition was calculated over a period of $3 \mathrm{~h}$, beginning at the point when the fluorescence intensity reached $10 \%$ of the maximum for that condition. This setpoint was determined to ensure that the data had made a significant increase from the baseline value and that rate calculations did not begin in the noise of the lag phase. All rate values were normalized to the rate of the huntingtin control $(100 \%)$. 


\subsubsection{Atomic force microscopy}

Htt-exon1(46Q) $(20 \mu \mathrm{M})$ was incubated with and without lipid vesicles $(200 \mu \mathrm{M}$ for a 10:1 lipid:protein ratio) at $37{ }^{\circ} \mathrm{C}$ and $1400 \mathrm{rpm}$ using an orbital mixer. At various time points, 2 $\mu \mathrm{L}$ aliquots of each condition were deposited on freshly cleaved mica for 1 min followed by a $200 \mu \mathrm{L}$ wash with $18 \mathrm{M} \Omega$ water and drying with a gentle stream of clean air. These samples were imaged using a Nanoscope V Multi-Mode scanning probe microscope (VEECO) equipped with a closed loop vertical engage J-scanner. Silicon-oxide cantilevers with a nominal spring constant of $40 \mathrm{~N} / \mathrm{m}$ and a resonance frequency of $300 \mathrm{kHz}$ were used. Scan rates were set to 1.99 $\mathrm{Hz}$ with cantilever drive frequencies at $10 \%$ of resonance. All images were analyzed using the Matlab image processing toolbox (MathWorks) as previously described. ${ }^{7,54}$

\subsubsection{Polydiacetylene lipid binding assay}

Polydiacetylene (PDA) assays were performed to measure the interaction between htt and lipid vesicles over time using reported protocols. ${ }^{55,56}$ In short, diacetylene monomers of 10,12tricosadiynoic acid and the pure lipid system of choice were mixed at a 2:3 molar ratio in a 4:1 chloforom/ethanol solution. The organic solvents were evaporated off with a gentle stream of nitrogen. Films were reconstituted in tris buffer $\left(70^{\circ} \mathrm{C}\right)$, extensively sonicated to promote mixing, and left at $4{ }^{\circ} \mathrm{C}$ overnight to allow for self-assembly into PDA/lipid vesicles. For each experiment, the vesicle solutions were equilibrated to room temperature and irradiated at $254 \mathrm{~nm}$ with constant stirring to polymerize the 10,12-tricosadiynoic acid, resulting in a royal blue solution that would display a colorimetric shift to red upon applied mechanical stress to the vesicles. PDA/lipid solutions were exposed to htt-exon1(46Q) $(20 \mu \mathrm{M})$ for $18 \mathrm{~h}$ at $30^{\circ} \mathrm{C}$. For each condition, the absorbance of the blue $(650 \mathrm{~nm})$ and red $(500 \mathrm{~nm})$ wavelengths were 
recorded by a SpectraMax M2 plate reader every 5 min for the duration of the experiment. A negative control consisted of equal volumes of neat buffer and PDA/lipid solution, while a positive control exposed vesicles to saturated $\mathrm{NaOH}(\mathrm{pH}=12)$ that stresses the system by increasing repulsion among lipid head groups, invoking a colorimetric response. ${ }^{57,58} \mathrm{The} \mathrm{NaOH}$ control can also be used to establish the sensitivity of the different lipid systems and used to normalize the colorimetric response. The $\% \mathrm{CR}$ was calculated for each condition using the following equation:

$$
\% C R=\left(\frac{P B_{0}-P B}{P B_{0}}\right) \times 100
$$

where the $\mathrm{PB}$ is defined as $\mathrm{A}_{\text {blue }} /\left(\mathrm{A}_{\text {blue }}+\mathrm{A}_{\text {red }}\right)$ for the negative control $\left(\mathrm{PB}_{0}\right)$ and sample condition (PB). Ablue and $\mathrm{A}_{\text {red }}$ refer to the measured absorbance at the blue and red wavelengths respectively.

\subsubsection{Electrospray ionization-mass spectrometry (ESI-MS)}

For MS experiments, lipid vesicles were formed as previously described, but using HPLC-grade water instead of tris buffer. Nt17 peptide $(10 \mu \mathrm{M})$ was incubated with the lipid of choice $(100 \mu \mathrm{M})$ at $37^{\circ} \mathrm{C}$ for 3 hours. All ESI-MS experiments were performed using a Q Exactive Hybrid Quadruple Orbitrap mass spectrometer (Thermo Fischer, San Jose, CA). Experiments were conducted using a $\mathrm{HESI}^{\mathrm{TM}}$ as the degree of lipid adduction observed is not an artifact of ionization method. ${ }^{59} \mathrm{MS}$ spectra were recorded in positive ion mode over a mass-tocharge $(\mathrm{m} / \mathrm{z})$ range of 400 to 4,000 . Samples were infused $(10 \mathrm{uL} / \mathrm{min})$ through a needle biased at 3,500 V above the instrument inlet. The parameters for the MS instrument were: $400{ }^{\circ} \mathrm{C}$ for the

capillary inlet temperature, $30^{\circ} \mathrm{C}$ for the analyzer temperature, $80 \mathrm{~V}$ for the S-lens assembly, 400 ms for the maximum injection time, $1 \times 10^{6}$ for the $\mathrm{AGC}$, and 70,000 for the MS resolution. 
Each spectrum was recorded (90 s) in triplicate; data were analyzed using Xcalibur 2.2 software suite (Thermo Scientific).

\subsubsection{Molecular dynamics system preparation ${ }^{1}$}

The structure was generated based on a previous solid-state NMR study (PDB 2DL2) and was modified in VMD. ${ }^{60}$ The standard N-terminal patch was applied, and the $\mathrm{C}$-terminus was amidated to mimic the absence of charge that would be present with the full huntingtin protein. The peptide was solvated using the solvate plugin in VMD. ${ }^{60}$ Randomly-coiled conformations were generated by heating from $300 \mathrm{~K}$ to $700 \mathrm{~K}$ with an $N V T$ ensemble in NAMD $2.13^{61}$ over 20 ps followed by $1 \mathrm{~ns}$ at $700 \mathrm{~K}$. A starting structure was randomly selected from among frames with the proper omega dihedral angles. Separately, 3 pure lipid bilayers of DMPC, POPC, and DOPC containing 150 lipids each were created, solvated, and ionized using the Optimal Membrane Generator in LOOS. ${ }^{62,63}$ The systems were equilibrated using an in-house protocol consisting of concurrent slow heating and release of restraints on the lipids. The randomly-coiled peptide was merged with each of the respective membrane systems and ion concentrations were adjusted to $150 \mathrm{mM}$ using the ionize plugin in VMD.${ }^{60}$ The merged systems were equilibrated in the $N P T$ ensemble for $1 \mathrm{~ns}$ at $310 \mathrm{~K}$ with the Langevin thermostat and $1 \mathrm{~atm}$ with the Langevin barostat in NAMD $2.13 .{ }^{61}$ The systems' sizes ranged from 49,000 to 53,000 atoms.

\subsubsection{WESTPA}

To facilitate sufficient sampling of binding and dissociation events of Nt17, the weighted ensemble approach via Weighted Ensemble Simulation Toolkit with Parallelization and Analysis (WESTPA) was utilized. ${ }^{64}$ WESTPA increases the sampling of rare events in MD while

\footnotetext{
${ }^{1}$ All molecular dynamics simulations and analysis were performed by Nicolas Frazee and Dr. Blake Mertz. 
maintaining equilibrium conditions. This is accomplished via a user-defined progress coordinate which describes the configurational space of the system. The progress coordinate is further subdivided into user-defined bins. WESTPA maintains a fixed number of simulations per bin tracking the probability that the bin will be populated over time.

WESTPA spawns several short simulations of time, $\tau$, after which it will evaluate the progress coordinate and add or remove simulations to maintain a constant number of simulations per bin. This approach facilitates a significantly increased fraction of simulations sampling the relevant space (i.e., bilayer proximal) and less time sampling the irrelevant space (i.e., diffusion in bulk solution).

Each lipid system was run in triplicate with 100 iterations per replica, a dynamics period $(\tau)$ of $20 \mathrm{ps}$, and five simulations per bin. NAMD $2.13^{61}$ was used for dynamics with the same parameters as the final equilibration step. A one-dimensional progress coordinate was defined as a function of the number of contacts between Nt17 and the bilayer. The progress coordinate, $C_{A B}$, was calculated as such

$$
C_{A B}=\sum_{\mathrm{i} \in \mathrm{A}} \sum_{\mathrm{j} \in \mathrm{B}} \frac{1}{1+\left(\frac{r_{i j}}{r_{0}}\right)^{6}}
$$

where $\mathrm{A}$ is the set of atoms in the lipids, $\mathrm{B}$ is the set of atoms in the peptide, $r_{i j}$ is the distance between the $i$ th lipid atom and $j$ th peptide atom, and $r_{0}$ is the cutoff distance of $3 \AA$. The binning scheme was designed to evenly divide up the $z$-distance between the starting position of the peptide and the center of the bilayer. The bin values are as follows: $0.0,0.4,0.8,1,1.5,2,3,4,5$, $7,10,15,25,40,70,120,200,300,500,650,800,1000,1250,1500,1750,2000,2250,2500$, $2750,3000,3250,3500,3750,4000,4250,4500,4750$, 'inf'. 


\subsubsection{Molecular dynamics analysis}
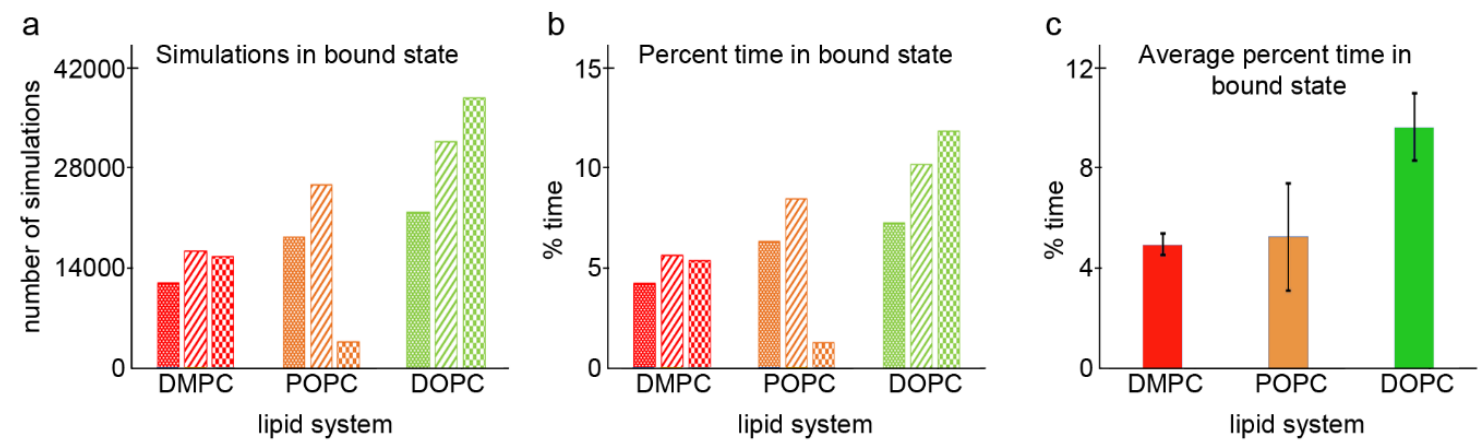

Figure 5.1 Propensity of binding events in WE simulations. a) Count of frames from each WESTPA trial by lipid system where Nt17 is considered to be in the "bound" state (pcoord greater than 120). b) The percent time from each trial of WESTPA where Nt17 is in the "bound" state. c) Average percent of time that Nt17 is in the bound state.

Analysis was performed on the subset of each weighted ensemble simulation that had a progress coordinate of 120 or greater at either the beginning or end of the iteration. A progress coordinate of 120 was chosen as a threshold based on visual inspection of the simulations where Nt17 was near the bilayer surface. These snapshots are referred to as "bound" throughout the rest of this paper. The distribution of bound frames can be seen in Fig. 5.1. Peptide-bilayer contacts: The number of contacts per frame was determined using a custom python script and pyLOOS $\frac{3,4}{3.4}$ by iterating through every atom in the peptide paired with every atom in the bilayer; those that were less than $4 \AA$ apart were considered a contact. Distance plots: $d_{z}$ is defined as the projection along the z-axis of the vector between the geometric average position of the atoms in each respective residue and the surface of the bilayer. This involved two calculations: 1) calculation of the length of the projection along the z-axis of the vector between the geometric average position of the atoms in each respective residue and the $\mathrm{P}$ atoms of the bilayer, 2) calculation of the average thickness of the bilayer by measuring the distance along the membrane normal between 
the $\mathrm{P}$ atoms in each respective leaflet of the bilayer. Half the bilayer thickness was subtracted from the z-projection to obtain $d_{z}$. Both measurements were made using the interdist tool in LOOS. ${ }^{62,63}$ Orientation of Nt17: The first principal axis of the peptide was measured against the bilayer normal using a custom python script and pyLOOS. ${ }^{62,63}$ Per residue distance dependent orientation: Distance was calculated similarly to $d_{z}$, however only the terminal heavy atom of the sidechain was considered for each respective residue. To measure the angle of the sidechain with respect to the bilayer normal, a vector was drawn from the $\mathrm{C}_{\alpha}$ carbon to the terminal heavy atom of each respective residue. Membrane maps: The membrane_map tool in $\operatorname{LOOS}^{62,63}$ was applied to each trial calculating the windowed height of the $\mathrm{P}$ atoms in the upper leaflet of the bilayer. Similar to $d_{z}$, half the bilayer thickness was subtracted from each windowed height to determine the displacement from the average. Membrane defects: Measurements of defects were based on the approach used by Voth and coworkers. ${ }^{65}$ With the trajectory centered in the xy-plane on the peptide, the measure sasa tool in $\mathrm{VMD}^{60}$ was used with a $3 \AA$ separation, -samples 300 , -restrict to a selection of $x / y<-13$ and $>13$ and $z>10$ (restricting the points to being on the surface of the bilayer), and -points to create a point-based surface of the bilayer. Points were saved every 100 frames. Points were categorized based on their z-position with respect to the plane of the $\mathrm{N}$ atoms in the upper leaflet (protrusions were above the plane; defects were below the plane). Networks were constructed by grouping points within $0.6 \AA$ of one another in the xy-plane. Areas were determined by first finding the maxima and minima of all the points for the frame to determine the rectangular area of the bilayer surface and then dividing the rectangular area into 2500 pixels of equal size. After this, the actual area was calculated by identifying how many pixels each network (i.e., defect/protrusion) occupied. Helicity: The phi and psi angles of the backbone were measured using the ramachandran tool in LOOS. ${ }^{62,63}$ A residue was considered 
helical if the previous and subsequent residues had a phi angle between -90 and -30 and a psi angle between -77 and $-17 .{ }^{66}$ Salt bridges: Salt bridges were measured using the salt bridge plugin with default values in $\mathrm{VMD}^{60}$ with modifications to accommodate measuring the $\mathrm{N}$ and $\mathrm{P}$ atoms of the lipid head groups.

\subsection{Results}

\subsubsection{Lipid membranes impact htt aggregation}

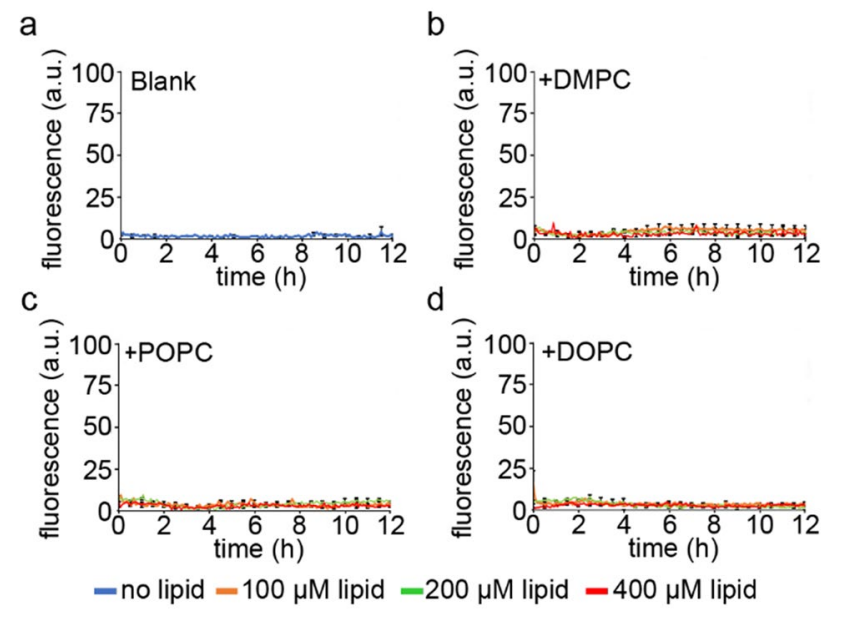
Figure 5.2 Lipid vesicles alone do not induce ThT fluorescence. ThT fluorescence assay controls of (a) neat buffer, (b) DMPC vesicles, (c) POPC vesicles, and (d) DOPC vesicles.

Experiments were performed with a glutathione S-transferase (GST)-htt-exon1(46Q) fusion protein. Factor Xa cleaves GST, initiating aggregation. The impact of DMPC, POPC, or DOPC lipid vesicles (5:1, 10:1, and 20:1 lipid:htt) on fibrillization was determined by ThT assays. While vesicles alone don't invoke a ThT signal (Fig. 5.2), each lipid system impacted htt fibrillization (Fig. 5.3). Compared to aggregation in the absence of lipids, DMPC accelerated fibrillization in a dose-dependent manner, as the rate of elongation increased by $29 \%, 88 \%$, and $114 \%$ at DMPC:htt ratios of 5:1, 10:1, and 20:1 respectively. The final fibril load, indicated by relative maximum fluorescence, increased in the presence of DMPC, with a maximum increase of 70\% at the 20:1 DMPC:htt ratio. POPC did not significantly alter 
fibrillization until the lipid:htt ratio increased to $20: 1$, which invoked a $26 \%$ increase in aggregation rate and a 10\% increase in maximum fluorescence. DOPC inhibited aggregation in a dose dependent manner, with the highest lipid:htt ratio reducing the maximum fluorescence $75 \%$. The aggregation rate decreased with increasing DOPC:htt ratios (39\% decrease at $5: 1,75 \%$ decrease at $10: 1,82 \%$ decrease at $20: 1)$.

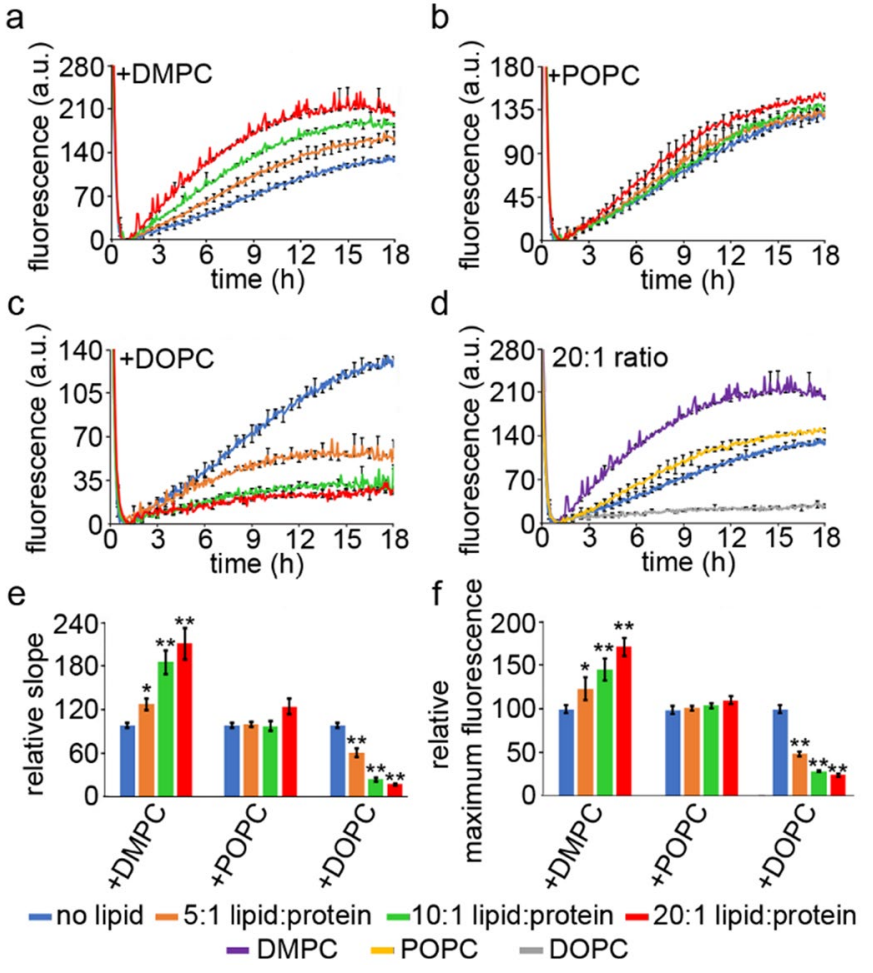

Figure 5.3 Varying the lipid tail alters how vesicles influence htt fibrilization. ThT aggregation assays for htt-exon1 (46Q) in the presence of (a) DMPC, (b) POPC, or (c) DOPC lipid vesicles. Htt-exon 1 concentration was $20 \mu \mathrm{M}$ and the lipid to htt molar ratio was $5: 1,10: 1$, or 20:1. (d) Direct comparison of the 20:1 ratio for each lipid system is shown. (e) The initial rate of aggregation and (f) the relative maximum fluorescence were determined with respect to the htt control. Error bars are provided for every sixth data point (30 min) and represent the standard error of the mean. One asterisk represents a $\mathrm{p}$ value of $<0.05$, and two asterisks represent a $\mathrm{p}$ value of $<0.01$.

As ThT predominately probes fibrillization, AFM assays were performed to analyze oligomer formation and aggregate morphology (Fig. 5.4). Htt-exon1(46Q) (20 $\mu \mathrm{M})$ was incubated alone or with DMPC, POPC, or DOPC vesicles at a molar ratio of 10:1 (lipid:htt). Incubations were sampled after 1, 3, 5, and $8 \mathrm{~h}$ and imaged by ex situ AFM. Images were analyzed to determine aggregate populations and morphologies. Oligomers were classified as features at least $1.0 \mathrm{~nm}$ in height occupying a surface area less $4,000 \mathrm{~nm}^{2}$ with an aspect ratio less than 3.0, indicating a globular structure. The $1 \mathrm{~nm}$ height threshold avoided counting 
artifacts associated with lipid backgrounds (as determined by control images, Fig. 5.5). Fibrils were defined as features with aspect ratios larger than 3.0 and occupying at least $4,000 \mathrm{~nm}^{2}$. To eliminate bias caused by varying fibril lengths and bundling, the percent surface covered by fibrils was used as a measure of fibril load.
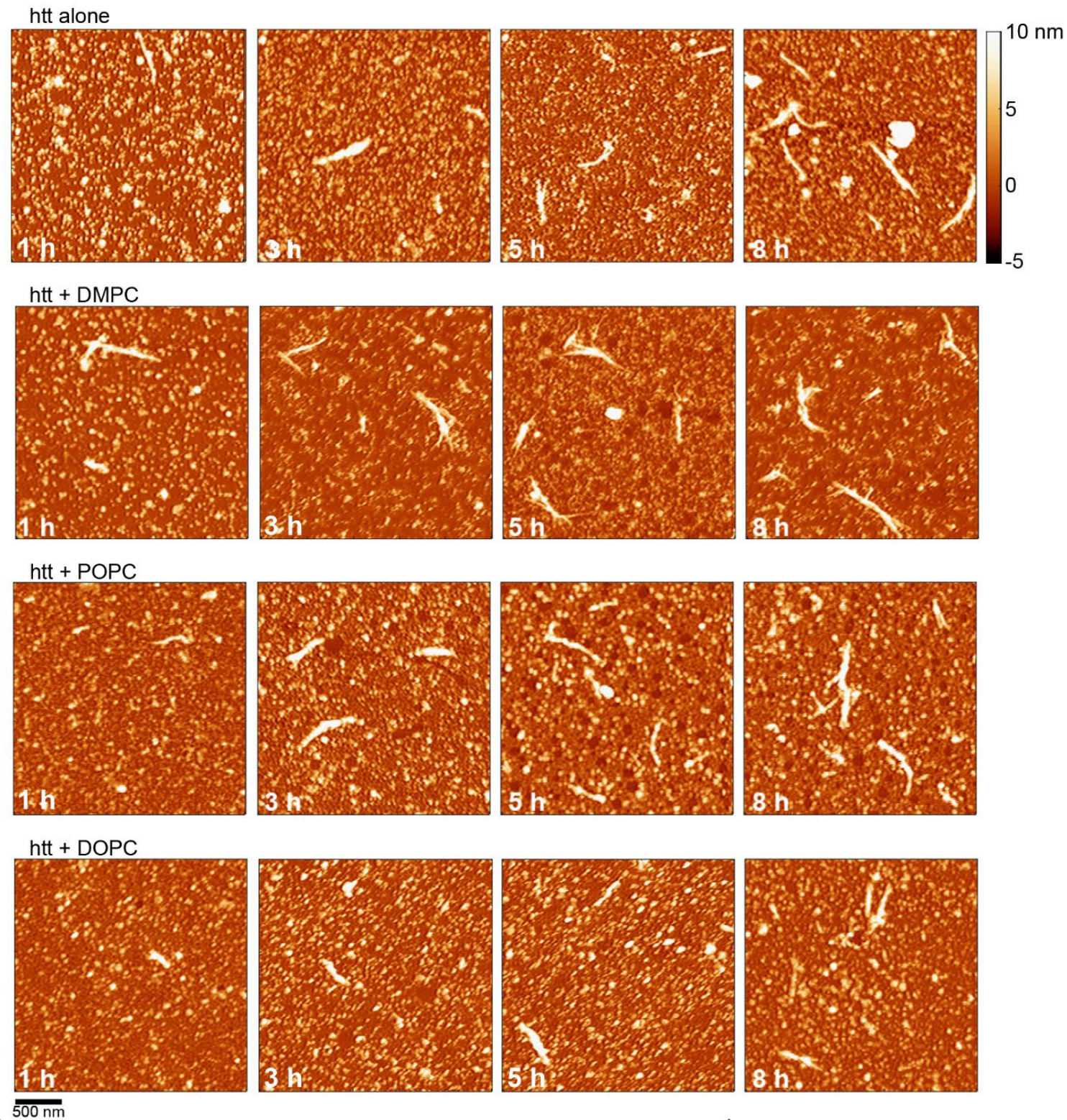

Figure 5.4 AFM images showing the effect of DMPC, POPC, and DOPC on htt-exon1(46Q) aggregation. Representative AFM images of $20 \mu \mathrm{M}$ htt-exon1(46Q) incubated alone, with DMPC, with POPC, or with DOPC lipid vesicles as a function of time (10:1 lipid:htt ratio). The color map is the same for all images. 

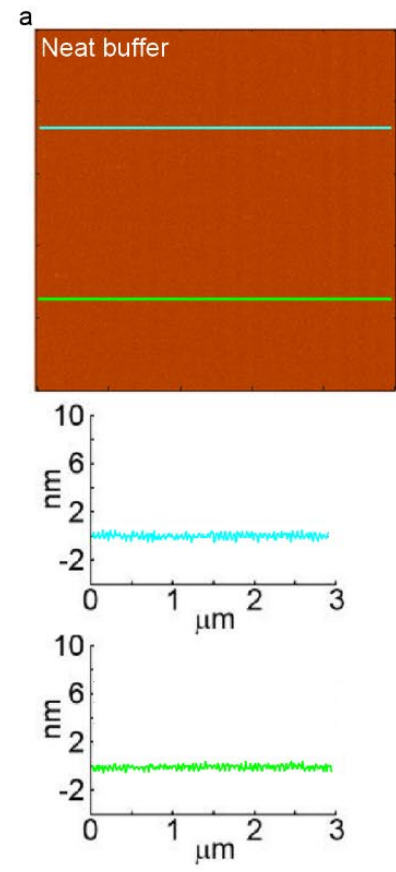
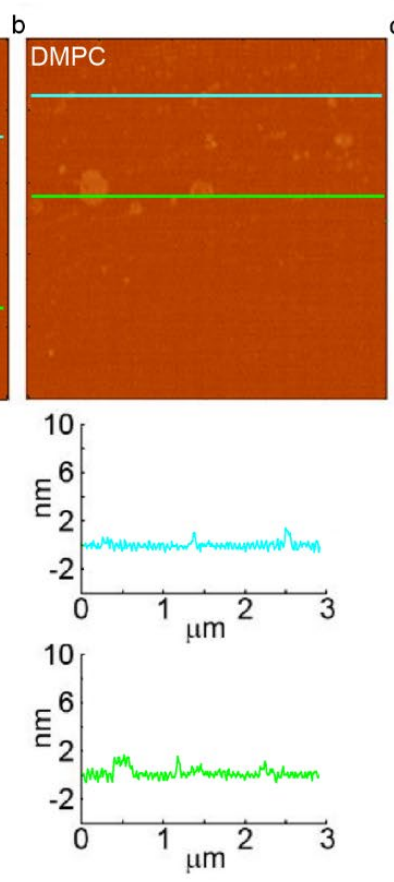
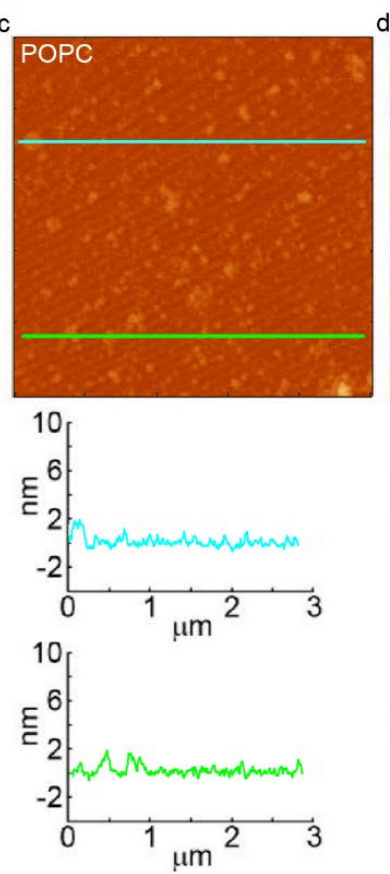
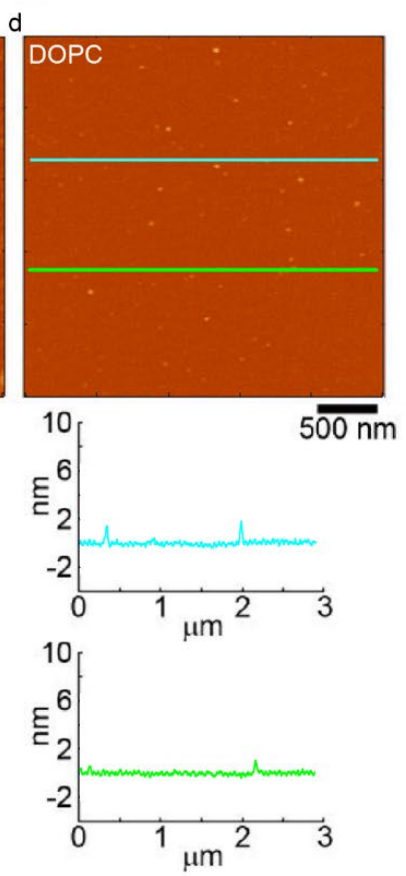

Figure 5.5 AFM images of backgrounds associated with different lipids. Representative AFM images of the backgrounds associated with (a) neat buffer, (b) DMPC lipid, (c) POPC lipid, (d) and DOPC lipid. The lines in each image correspond to the height profiles provided below each image.

Htt-exon1(46Q) (no lipid) forms oligomers within $1 \mathrm{~h}$ with a peak population after $3 \mathrm{~h}$ (Fig. 5.6a). A few fibrils were present at $1 \mathrm{~h}$, but these became longer and more numerous with time, occupying a larger surface area. The oligomer population decreased with increasing fibril content. With DMPC, there was a slight increase in oligomers at $1 \mathrm{~h}$ compared with control. After peaking at $3 \mathrm{~h}$, the number of oligomers observed with DMPC was reduced at 5 and $8 \mathrm{~h}$ compared to control $(\mathrm{p}<0.05)$. DMPC enhanced fibrillization, with a clear increase in coverage at $5 \mathrm{~h}$ compared with control ( $\mathrm{p}<0.05$, Fig. 5.6b). The addition of POPC vesicles significantly reduced the htt oligomer population at 3, 5, and $8 \mathrm{~h}$ (Fig. 5.6a). Despite this reduction in oligomers, the extent of fibrillization was comparable to control (Fig. 5.6b). Aggregate populations were altered by DOPC (Fig. 5.6a-b). DOPC promoted and stabilized oligomers as 
their population surpassed control at $1 \mathrm{~h}$, remaining constant over $8 \mathrm{~h}$. This oligomer stability was accompanied by a significant decrease in fibril content. Fibril content observed with each lipid system was consistent with ThT assays.
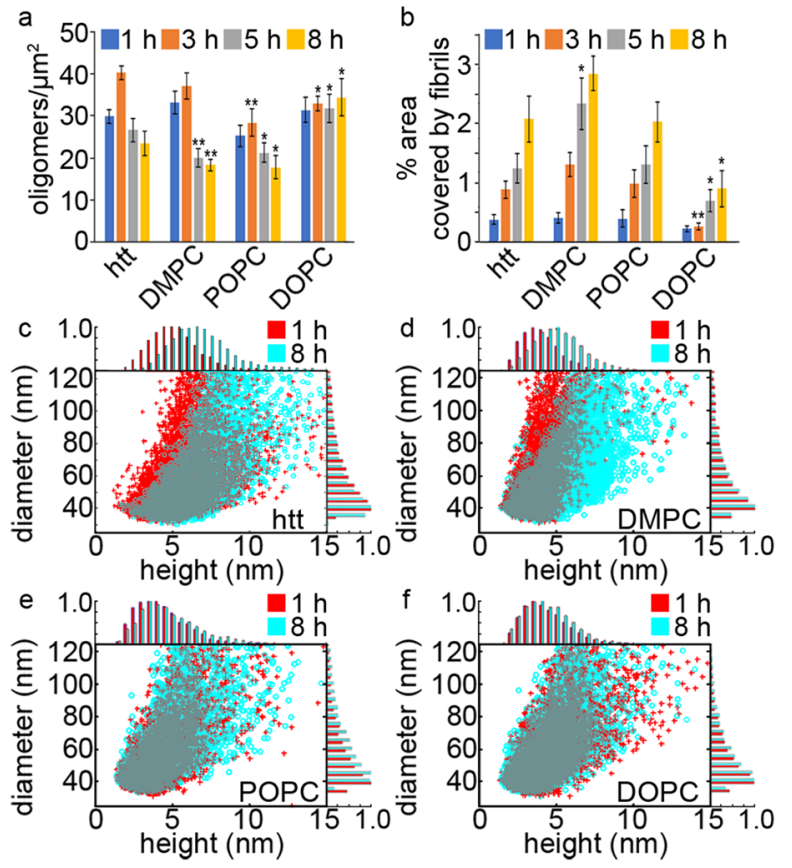

Figure 5.6 AFM analysis of the impact of DMPC, POPC, or DOPC lipid vesicles on htt-exon1(46Q) aggregation (10:1 lipid:htt ratio). (a) Analysis of the number of oligomers per unit area as a function of time for each condition. (b) Statistical analysis of the percent of each image area covered by fibrils as a condition of time. (c-f) Comparison of the height and diameter of oligomers formed at 1 $\mathrm{h}$ (red) and $8 \mathrm{~h}$ (blue) in the presence of (c) no lipid, (d) DMPC, (e) POPC, and (f) DOPC vesicles. One asterisk represents a $p$ value of $<0.05$, and two asterisks represent a $\mathrm{p}$ value of $<0.01$.

Next, the morphologies of htt aggregates were compared. While varying rates of fibrillization result in different fibril contour lengths, the average height along the fibril contour was unchanged, suggesting that the underlying structure of fibrils was not altered by each lipid (Fig. 5.7). However, lipids altered oligomer morphology (Fig. 5.6c-f). Without lipids, oligomers increased in size with time (Fig. 5.6c). Specifically, oligomers had a mode height of $\sim 4-5 \mathrm{~nm}$ after $1 \mathrm{~h}$, but this grew to $6-8.5 \mathrm{~nm}$ at $8 \mathrm{~h}$ with an increase in heterogeneity. A shift from smaller to larger oligomers occurred in the presence of DMPC, but the $8 \mathrm{~h}$ population displayed less heterogeneity and a smaller mode height ( 4.5-6.5 nm, Fig. 5.6d). With both POPC and DOPC, oligomers initially were larger (mode height of 4-5 $\mathrm{nm}$ for both conditions); however, oligomer 
morphology was stable, displaying extensive overlap in the correlation plots between 1 and 8 h (Fig. 5.6e-f).
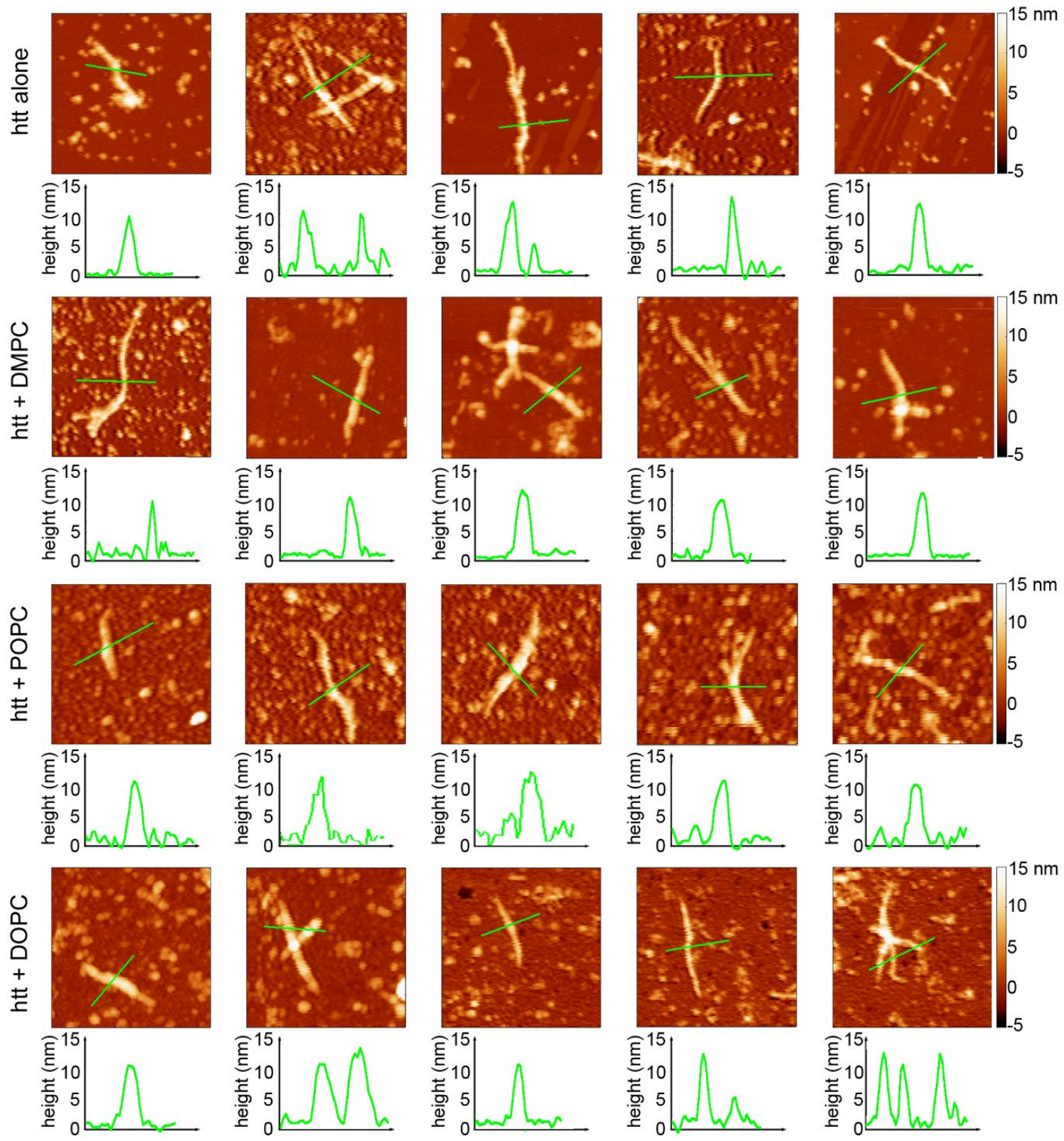

Figure 5.7 Htt-exon1(46Q) fibrils formed in the presence of DMPC, POPC, and DOPC vesicles are similar in morphology to those formed in the absence of lipid. AFM images comparing the morphology of htt-exon1(46Q) fibrils formed in the absence of lipid, or when incubated with DMPC, POPC, or DOPC lipid vesicles. The color lines in each image correspond to the height profiles directly below each image. The color map is the same for all images. 


\subsubsection{Saturation in lipid tails influence $\mathrm{htt} / \mathrm{membrane}$ interactions}

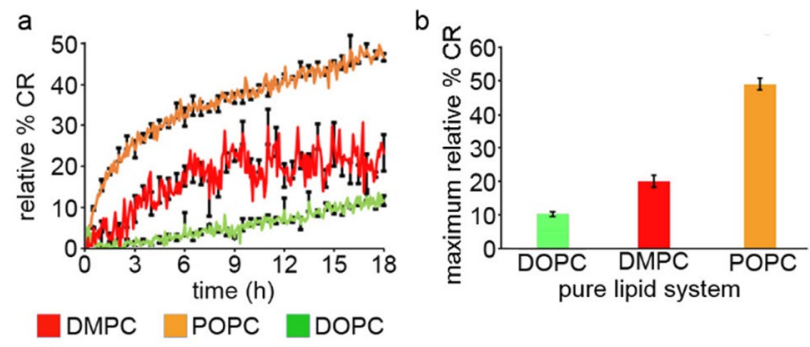

Figure 5.8 Htt-exon1(46Q) membrane interaction is altered by properties associated with the lipid tail. (a) Lipid binding assays for PDA/ DMPC, PDA/POPC, and PDA/DOPC vesicles exposed to $20 \mu \mathrm{M}$ httexon1(46Q). Error bars are provided for every sixth data point and represent the standard error of the mean. (b) The average maximum relative $\% \mathrm{CR}$ for each PDA/lipid system.

The varying impact of the lipids systems on aggregation suggests differences in htt/lipid interaction, so polydiacetylene (PDA)/lipid affinity assays were performed using DMPC, POPC, or DOPC as a first assessment of htt/lipid interaction (Fig. 5.8). PDA/lipid vesicles undergo a colorimetric shift (blue to red) as protein interaction with the vesicles results in a mechanical strain on the PDA polymer backbone. By monitoring blue $(640 \mathrm{~nm})$ and red $(500 \mathrm{~nm})$ absorbances of the vesicles upon exposure to htt-exon1(46Q), a percent colorimetric response (\% $\mathrm{CR})$ can be calculated that directly correlates to the extent of htt/lipid interaction. To enable direct comparison between the different lipid systems, the reported \% CR are normalized against a standard dose of $\mathrm{NaOH}$. Based on this assay, the relative affinity of htt-exon1(46Q) for the different lipid systems was POPC > DMPC > DOPC. Htt quickly bound POPC vesicles, invoking a greater \% CR within $3 \mathrm{~h}$ than was observed for the other lipid systems over $18 \mathrm{~h}$. After this initial jump, the \% CR continued to increase. With DMPC/PDA vesicles, the $\% \mathrm{CR}$ steadily rose for $\sim 9 \mathrm{~h}$ until a steady state interaction of $\sim 20 \% \mathrm{CR}$ was observed. It took over $3 \mathrm{~h}$ of htt exposure before DOPC/PDA vesicles elicited an observable \% CR; however, once initiated the $\% \mathrm{CR}$ continued to gradually increase for the duration of the experiment. The overall trend 
did not correlate with degree of lipid tail unsaturation, and the differences between the $\% \mathrm{CR}$ curves suggests mechanistic differences in the interaction of htt with these lipid systems.

\subsubsection{Nt17 forms a variety of complexes with lipids}

As Nt17 facilitates htt-exon1 lipid interactions, ESI-MS was utilized to determine complexes formed with Nt17 peptides and the lipid systems (Fig. 5.9, Table 5.1). Nt17 (10 $\mu \mathrm{M})$ was incubated alone or with DMPC, POPC, or DOPC vesicles (10:1 lipid:peptide ratio) for $3 \mathrm{~h}$ before analysis. Relative abundance was determined in relation to the doubly-charged monomer peptide ion $\left([\mathrm{M}+2 \mathrm{H}]^{2+}\right.$ ion at $m / z$ 988). Without lipids, several Nt17 ions were present (Fig. 5.9a). The $[\mathrm{M}+2 \mathrm{H}]^{2+}$ ions at $m / z 988$ were the predominate species, followed by the $[\mathrm{M}+3 \mathrm{H}]^{3+}$ ions at $m / z 988$ and the $[\mathrm{M}+\mathrm{H}]^{1+}$ ions at $m / z$ 1974. Dimer, trimer, and tetramer ions for the Nt17 peptide $(\mathrm{m} / \mathrm{z} 1317,1482$, and 1580 , respectively) were observed at significantly lower abundances. The lyophilized peptide contained sodium, resulting in sodium-containing ions.

A heterogeneous mixture of ions corresponding to Nt17-lipid complexes were observed with all three lipid systems (Fig. 5.9b-d, Table 5.1). Complexes contained monomeric to trimeric Nt17 and up to 6 lipids, with the makeup and abundance of complexes varying between lipid systems (Fig. 5.9e-h). The relative intensity of ions for each lipid system decreased with multimers of increasing size. However, lipid content in complexes with each system varied. The number of distinct complexes identified was similar for each lipid system (Table 5.1), but the total relative abundance paralleled the PDA assay (POPC $>$ DMPC $>$ DOPC, Fig. 5.9e). That said, complexes of the same composition but different charge states were observed. POPC had the lowest number of Nt17-lipid combinations despite having the highest relative abundance of ions while DOPC had the most distinct combinations but the lowest relative abundance. 

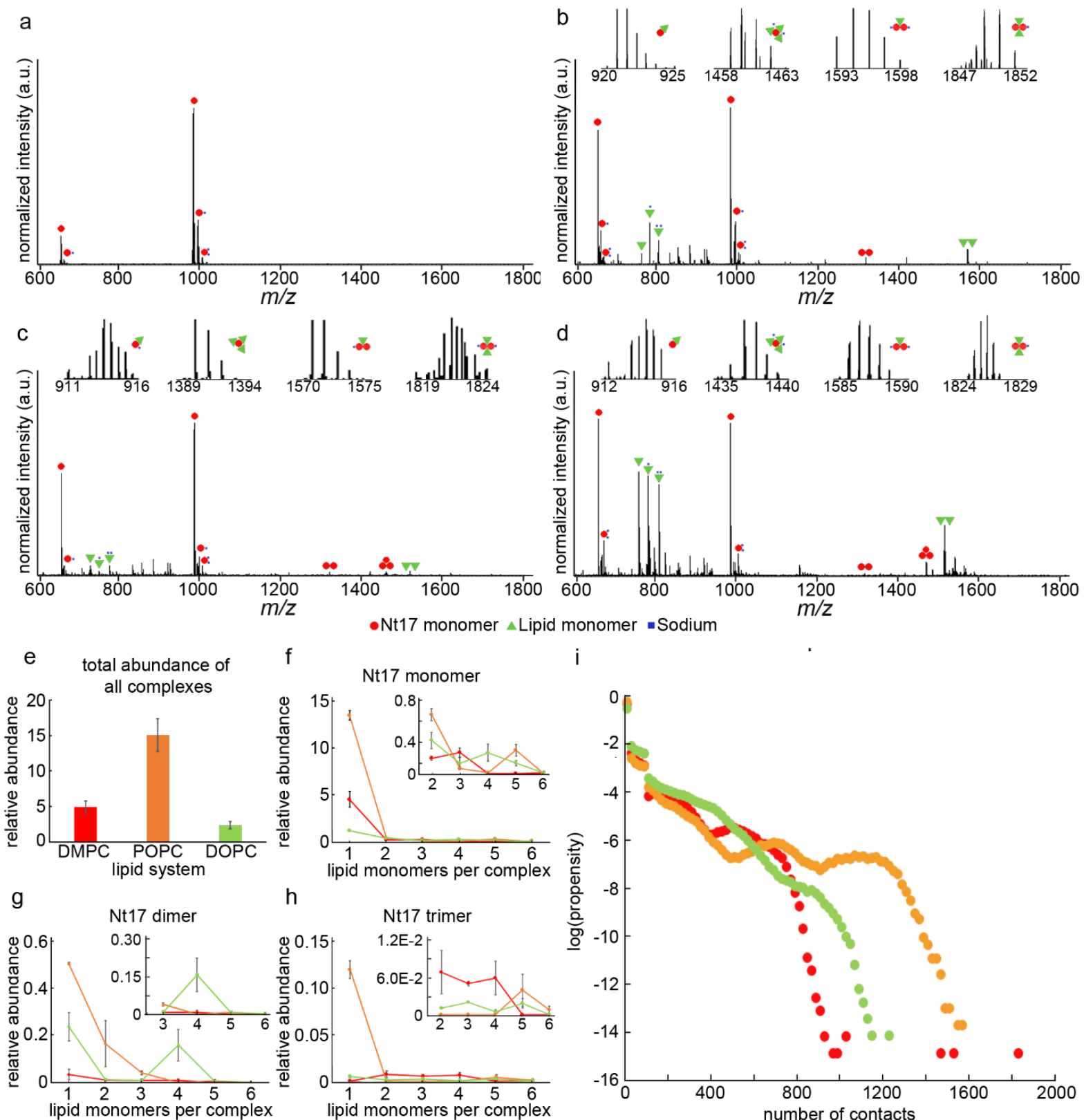

i

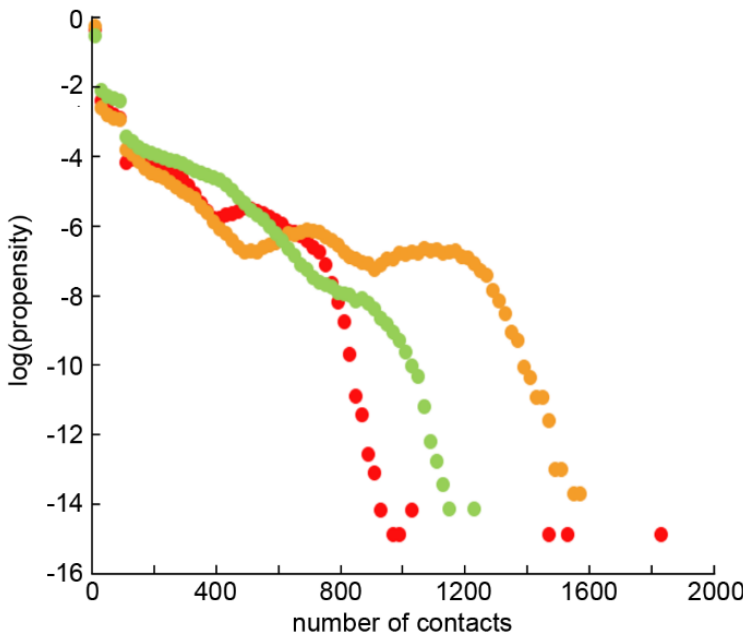

DMPC $\triangle P O P C \triangle D O P C$

Figure 5.9 Nt17 forms complexes with DMPC, POPC, or DOPC vesicles of different makeup and abundance as a result of varying molecular contacts. The composition and abundance of adducts formed with Nt17 and different lipids was analyzed via ESI-MS. Nt17 was incubated (a) alone or with (b) DMPC, (c) POPC, or (d) DOPC lipid vesicles. (e) The total abundance of peptide-lipid complexes was compared across all systems, as well as the abundance of complexes by lipid content with Nt17 (f) monomers, (g) dimers, and (h) trimers. (i) The total molecular contacts made between Nt17 and DMPC, POPC, or DOPC lipid bilayers in MD simulations. 
Table 5.1 Assigned ions for Nt17 incubated with DMPC, POPC, or DOPC lipid vesicles along with their mass to charge ratios.

\begin{tabular}{|c|c|c|c|c|c|c|c|c|}
\hline $\operatorname{lon}^{a}$ & $\mathrm{~m} / \mathrm{z}$ & $\begin{array}{c}\text { Charge } \\
\text { State }\end{array}$ & $\operatorname{Ion}^{a}$ & $\mathrm{~m} / \mathrm{z}$ & $\begin{array}{c}\text { Charge } \\
\text { State }\end{array}$ & $\operatorname{Ion}^{\mathrm{a}}$ & $\mathrm{m} / \mathrm{z}$ & $\begin{array}{c}\text { Charge } \\
\text { State }\end{array}$ \\
\hline $\mathrm{Nt17}+$ 1DMPC & 2652.99 & 1 & $\mathrm{Nt17}+1 \mathrm{POPC}$ & 2734.63 & 1 & Nt17 + 1 DOPC & 2760.64 & 1 \\
\hline Nt17 + 1DMPC & 1327.00 & 2 & $\mathrm{Nt} 17+1 \mathrm{POPC}$ & 912.21 & 3 & Nt17 + 1DOPC & 1380.82 & 2 \\
\hline Nt17 + 1DMPC & 885.00 & 3 & $\mathrm{Nt17}+1 \mathrm{POPC}$ & 1367.82 & 2 & $\mathrm{Nt17}+2 \mathrm{DOPC}$ & 1773.62 & 2 \\
\hline $\mathrm{Nt} 17+2 \mathrm{DMPC}$ & 1665.47 & 2 & $\mathrm{Nt17}+2 \mathrm{POPC}$ & 1747.11 & 2 & $\mathrm{Nt17}+3 \mathrm{DOPC}$ & 2166.42 & 2 \\
\hline $\mathrm{Nt17}+2 \mathrm{DMPC}$ & 1110.98 & 3 & $\mathrm{Nt} 17+2 \mathrm{POPC}$ & 1165.41 & 3 & $\mathrm{Nt17}+3 \mathrm{DOPC}$ & 1444.61 & 3 \\
\hline $\mathrm{Nt} 17+3 \mathrm{DMPC}$ & 2004.45 & 2 & $\mathrm{Nt17}+3 \mathrm{POPC}$ & 2126.91 & 2 & $\mathrm{Nt17}+4 \mathrm{DOPC}$ & 2559.22 & 2 \\
\hline $\mathrm{Nt17}+3 \mathrm{DMPC}$ & 1336.96 & 3 & $\mathrm{Nt} 17+3 \mathrm{POPC}$ & 1418.60 & 3 & $\mathrm{Nt} 17+5 \mathrm{DOPC}$ & 2952.02 & 2 \\
\hline $\mathrm{Nt} 17$ + 4DMPC & 2343.42 & 2 & $\mathrm{Nt17}+4 \mathrm{POPC}$ & 2506.70 & 2 & $\mathrm{Nt17}+6 \mathrm{DOPC}$ & 2230.21 & 3 \\
\hline $\mathrm{Nt} 17+5 \mathrm{DMPC}$ & 2682.40 & 2 & $\mathrm{Nt} 17+4 \mathrm{POPC}$ & 1671.80 & 3 & $2 \mathrm{Nt} 17+1 \mathrm{DOPC}$ & 2367.84 & 2 \\
\hline $\mathrm{Nt} 17+6 \mathrm{DMPC}$ & 3021.37 & 2 & $\mathrm{Nt17}+5 \mathrm{POPC}$ & 2897.99 & 2 & $2 \mathrm{Nt17}+1 \mathrm{DOPC}$ & 1578.89 & 3 \\
\hline $\mathrm{Nt} 17+6 \mathrm{DMPC}$ & 2014.91 & 3 & $\mathrm{Nt17}+5 \mathrm{POPC}$ & 1925.00 & 3 & $2 \mathrm{Nt} 17+2 \mathrm{DOPC}$ & 2760.64 & 2 \\
\hline $2 \mathrm{Nt} 17+1 \mathrm{DMPC}$ & 2314.02 & 2 & $2 \mathrm{Nt} 17+1 \mathrm{POPC}$ & 2354.84 & 2 & $2 \mathrm{Nt} 17+3 \mathrm{DOPC}$ & 3153.44 & 2 \\
\hline $2 \mathrm{Nt} 17+1 \mathrm{DMPC}$ & 1543.01 & 3 & $2 \mathrm{Nt} 17+1 \mathrm{POPC}$ & 1570.22 & 3 & $2 \mathrm{Nt} 17+4 \mathrm{DOPC}$ & 2364.49 & 3 \\
\hline $2 \mathrm{Nt} 17+2 \mathrm{DMPC}$ & 2652.49 & 2 & $2 \mathrm{Nt} 17+2 \mathrm{POPC}$ & 2734.13 & 2 & $2 \mathrm{Nt} 17+5 \mathrm{DOPC}$ & 2626.36 & 3 \\
\hline $2 \mathrm{Nt} 17+3 \mathrm{DMPC}$ & 2991.47 & 2 & $2 \mathrm{Nt} 17+2 \mathrm{POPC}$ & 1823.42 & 3 & $2 \mathrm{Nt} 17+6 \mathrm{DOPC}$ & 2888.22 & 3 \\
\hline $2 \mathrm{Nt} 17+3 \mathrm{DMPC}$ & 1994.98 & 3 & $2 \mathrm{Nt} 17+3 \mathrm{POPC}$ & 3113.93 & 2 & $3 \mathrm{Nt} 17+1 \mathrm{DOPC}$ & 2236.90 & 3 \\
\hline $2 \mathrm{Nt} 17+4 \mathrm{DMPC}$ & 3330.44 & 2 & $2 \mathrm{Nt} 17+3 \mathrm{POPC}$ & 2076.62 & 3 & $3 \mathrm{Nt17}+2 \mathrm{DOPC}$ & 2498.77 & 3 \\
\hline $2 \mathrm{Nt} 17+4 \mathrm{DMPC}$ & 2220.96 & 3 & $2 \mathrm{Nt} 17+5 \mathrm{POPC}$ & 2583.01 & 3 & $3 \mathrm{Nt17}+3 \mathrm{DOPC}$ & 2760.64 & 3 \\
\hline $3 \mathrm{Nt17}+2 \mathrm{DMPC}$ & 2427.01 & 3 & $3 \mathrm{Nt} 17+1 \mathrm{POPC}$ & 2235.90 & 3 & $3 \mathrm{Nt17}+4 \mathrm{DPOC}$ & 3022.51 & 3 \\
\hline $3 \mathrm{Nt17}+3 \mathrm{DMPC}$ & 2652.99 & 3 & $3 \mathrm{Nt} 17+5 \mathrm{POPC}$ & 3241.02 & 3 & $3 \mathrm{Nt17}+5 \mathrm{DOPC}$ & 3284.37 & 3 \\
\hline $3 \mathrm{Nt} 17+4 \mathrm{DMPC}$ & 2878.97 & 3 & $3 \mathrm{Nt} 17+6 \mathrm{POPC}$ & 3494.22 & 3 & & & \\
\hline
\end{tabular}

Monomeric Nt17 complexes were observed with up to 6 DMPC lipid monomers, with the most abundant complexes containing 1 DMPC lipid (relative abundance of 4.44\%), 3 DMPC lipids (0.22\%), and then 2 DMPC lipids (0.16\%, Fig. 5.9f). Dimeric and trimeric Nt17-DMPC complexes were significantly less abundant, containing only up to 4 DMPC lipids (Fig. 5.9g-h). Complexes of trimeric Nt17 with 1 DMPC lipid were nonexistent. Instead, there was a high abundance of trimeric Nt17 complexes with multiple DMPC lipids compared to other systems. 
The total relative intensities of Nt17-DMPC complex ions was $4.92 \%$. In contrast, the total relative intensity of Nt17- POPC complex ions was much higher (15.05\%, Fig. 5.9e). This large population was predominately comprised of monomeric Nt17-POPC complexes (relative intensity of $14.21 \%$, Fig. 5.9f). While eleven distinct monomeric Nt17-lipid ions were observed with both DMPC and POPC, monomeric Nt17-POPC complexes only contained up to 5 POPC lipids. Dimeric and trimeric Nt17-POPC complexes were less abundant (Fig. 5.9g-h). Nt17 dimers complexed with either 1, 2, 3, or 5 POPC lipids; Nt17 trimers complexed with 1, 5, or 6 POPC lipids. Nt17-DOPC complexes were the least abundant, reaching a total relative abundance of only $2.38 \%$ (Fig. 5.9e). The most abundant complex consisted of monomeric Nt17 with 1 DOPC lipid (1.17\%, Fig. 5.9f). Dimeric and trimeric Nt17-DOPC complexes were observed with up to 6 DOPC lipids or 5 DOPC lipids respectively Fig. 5.9g-h).

To complement these native MS studies, the interaction of Nt17 with DMPC, POPC, or DOPC was investigates using weighted ensemble molecular dynamic simulations. There appears to be a rough correlation between the abundance of Nt17/lipid complexes observed by MS and the total molecular contacts between Nt17 and the different lipid membranes (Fig. 5.9I). The propensity of Nt17 to form 800 or fewer contacts with each lipid system was similar. Nt17 had the highest propensity to form a large number $(>1,400)$ of contacts with POPC, consistent with the significantly larger abundance of observed complexes. While Nt17 had a higher propensity to form an intermediate number of contacts $(800$ to 1,400$)$ with DOPC compared with DMPC, Nt17 formed larger contacts $(>1,400)$ with DMPC that were not observed with DOPC. The observation of a slightly larger number of complexes comprised of Nt17/DMPC compared to Nt17/DOPC suggests that these larger contacts lead to high stability. Based on this, the MD simulations were further explored to better understand the binding of Nt17 to these lipid systems. 


\subsubsection{Effect of lipid type on binding of $\mathrm{Nt}^{2} 7^{2}$}

When examining overall binding, Nt17 possesses distinct orientations for each lipid type (Fig. 5.10 and Fig. 5.11). Bound complexes to DMPC are dominated by partitioning of the Nterminus into the bilayer surface. This Nt17 segment is a mixture of nonpolar and polar residues (MATL), which individually are not too bulky to access the bilayer interior sandwiched between two relatively densely-packed headgroup regions $\left(\mathrm{A}_{\mathrm{DMPC}}=60.6 \AA 2 / \text { lipid }\right)^{67}$ (Fig. 5.10). The Nt17 C-terminus is mainly dissociated from the DMPC bilayer, with a small sub-population that is less than $10 \AA$ from the surface. In contrast, Nt17 strongly binds POPC via the C-terminus; F17 partitions almost $5 \AA$ into the headgroup region, with an extensive segment (F11, E12, S13, L14, K15, and S16) remaining less than $10 \AA$ from the bilayer surface. This strong interaction potentially stems from the opportunity for a bulkier, nonpolar sidechain with conformational flexibility (i.e., F17) probing larger defects associated with a larger area per lipid (68.3 22 / lipid). ${ }^{68}$ Although there is a small fraction of the middle segment of Nt17 within $15 \AA$ of the bilayer, in general, the $\mathrm{N}$-terminal portion is dissociated from the membrane, beginning with A10 and getting progressively further from the bilayer as one proceeds towards M1. For both DMPC and POPC, one of the Nt17 termini binds the membrane in a conformation that is orthogonal to the bilayer surface (Fig. 5.10a, 5.10c). However, with DOPC, nearly all Nt17 residues interact with the membrane (Fig. 5.10b), leading to a parallel orientation of the Nt17 principal axis with respect to the bilayer. Additionally, not a single residue binds most strongly, likely stemming from DOPC having the largest area per lipid of all the systems tested $(72.2 \AA 2 / \text { lipid })^{69}$ and leading to a larger defect population that can be utilized by Nt17.

\footnotetext{
${ }^{2}$ All molecular dynamics simulations and analysis were performed by Nicolas Frazee and Dr. Blake Mertz. 
a
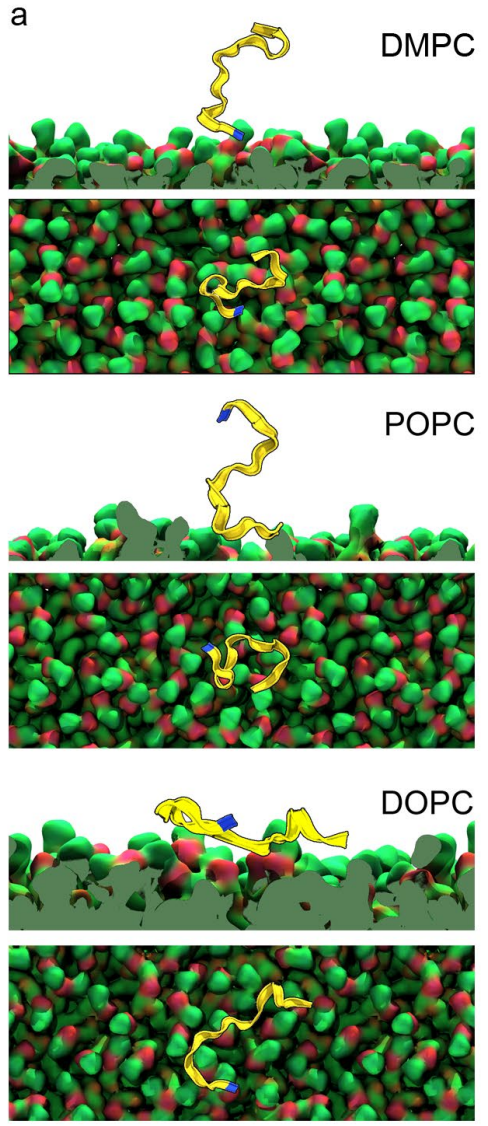

b
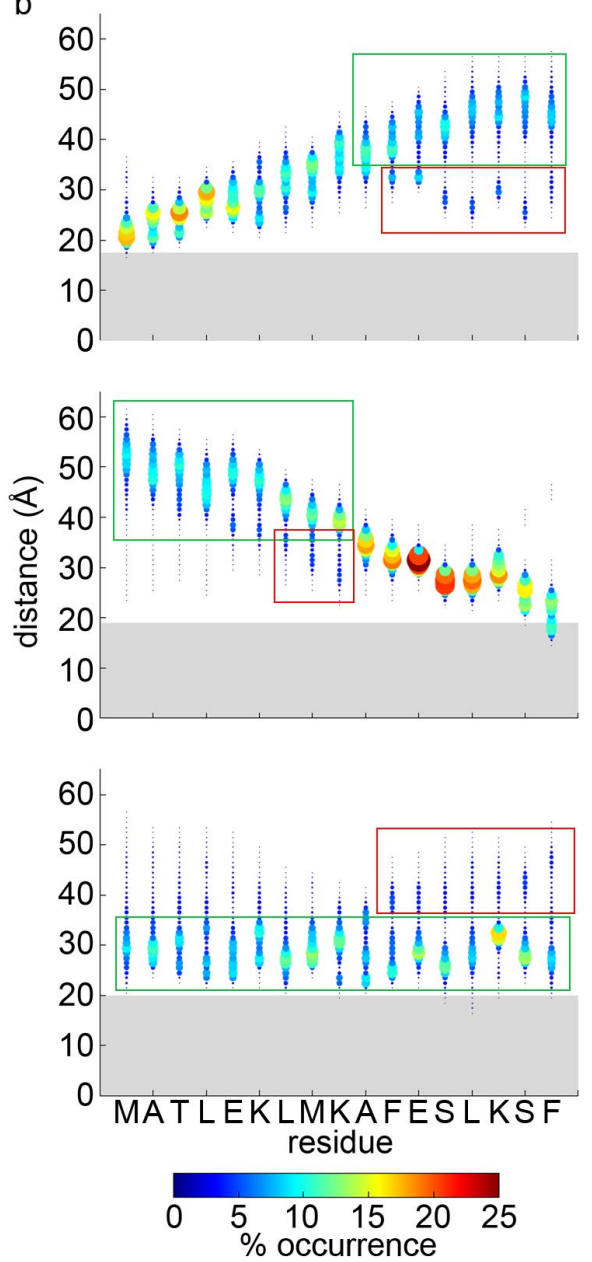
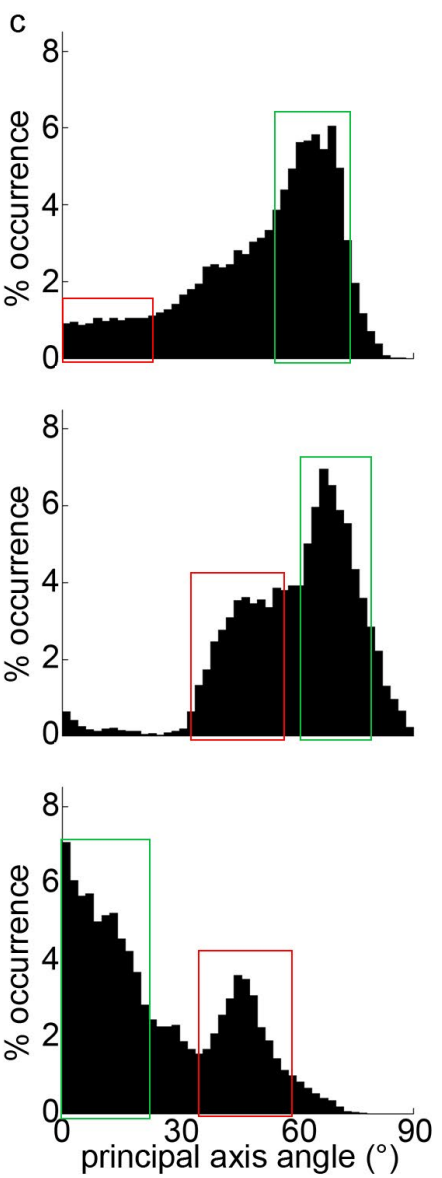

Figure 5.10 Lipid tails affect the binding orientation of Nt17 to lipid bilayers. a)

Representative snapshots of the bound state for each lipid system from weighted ensemble simulations. Yellow ribbon: $\mathrm{Nt17}$, blue ribbon: first residue of $\mathrm{Nt17}$, red/green: lipid membrane. Water and ions omitted for clarity. b) Binned z-distance between the geometric centers of the membrane and residues of $\mathrm{Nt} 17$ for frames in the bound state. Point size and color correspond to percent occurrence. c) Binned angle between the principal axis of the peptide and the bilayer surface. For $b$ and $c$, green boxes represent data associated with the most likely orientation of $\mathrm{Nt17}$ on the bilayer, shown in panel a. Red boxes represent data associated with a secondary population, shown in Figure 5.11. 

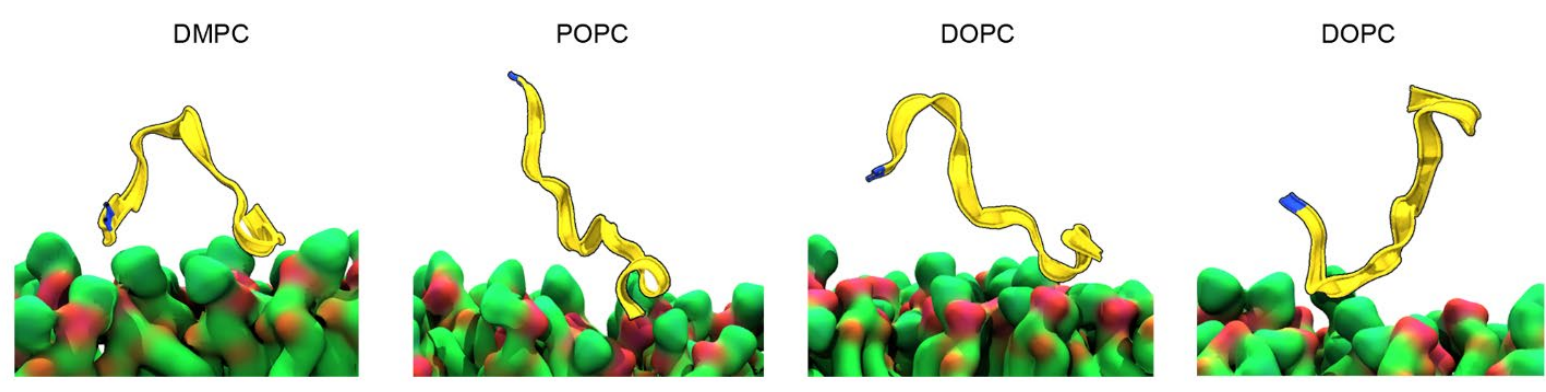

Figure 5.11 Secondary populations of Nt17 orientation on the lipid bilayers were observed for all systems. Representative snapshots of less commonly observed bound structures for each lipid system from weighted ensemble simulations. Two unique conformations are shown for DOPC.

\subsubsection{Relationship between sidechain orientation and binding of $\mathrm{Nt17}$}

Closer examination of residue-specific interactions of $\mathrm{Nt} 17$ with the bilayer surface reveals that, in most instances, sidechain orientation lies either parallel or away from the bilayer. For DMPC, T3 and K6 are notably parallel to the bilayer ( $\sim 90$ deg), likely ruling out the potential for non-bonded interactions (hydrogen bonding for T3 and salt bridge formation for K6) with PC headgroups to drive formation of stable binding conformations (Fig. 5.12 and Fig. 5.13). Aromatic (F11 and F17) and charged (K15) residues in the C-terminal half of Nt17 occupy 2-3 areas of conformational space, indicating that the dissociated portion of $\mathrm{Nt} 17$ is restricted to regions that allow it to bind via the N-terminus. Binding of Nt17 to POPC is much different: F17 partitions into the headgroup region nearly antiparallel to the membrane normal, most likely between acyl chains near the triglyceride backbone. This serves to restrict F11 and K15 (proximal to F17) parallel to the membrane normal (90 deg), whereas other residues (T3, K6, and K9) broadly sample orientations ranging from parallel ( $0 \mathrm{deg})$ to antiparallel (180 deg) (Fig.

5.12). For DOPC, there is a correlation between the distance of a given residue from the bilayer surface and sidechain orientation for nonpolar or slightly polar residues. Specifically, L7, A10, 
S13, and L14 sample conformations partitioned into the bilayer (Fig. 5.13). Additionally, there is a noticeable population for each lysine residue ( $\mathrm{K} 6, \mathrm{~K} 9$, and $\mathrm{K} 15)$ oriented towards the bilayer and close enough to potentially form a salt bridge with the negatively-charged phosphate moiety in the PC headgroup. This, in conjunction with the overall closer positioning, suggests binding of Nt17 to DOPC is driven by a combination of charge-charge interactions and greater accessibility for peptide partitioning. With DOPC, a threshold has been reached with respect to area per lipid and Nt17 binding, in that there is a sufficient population and size of defects to allow the entire peptide to partition into the bilayer.
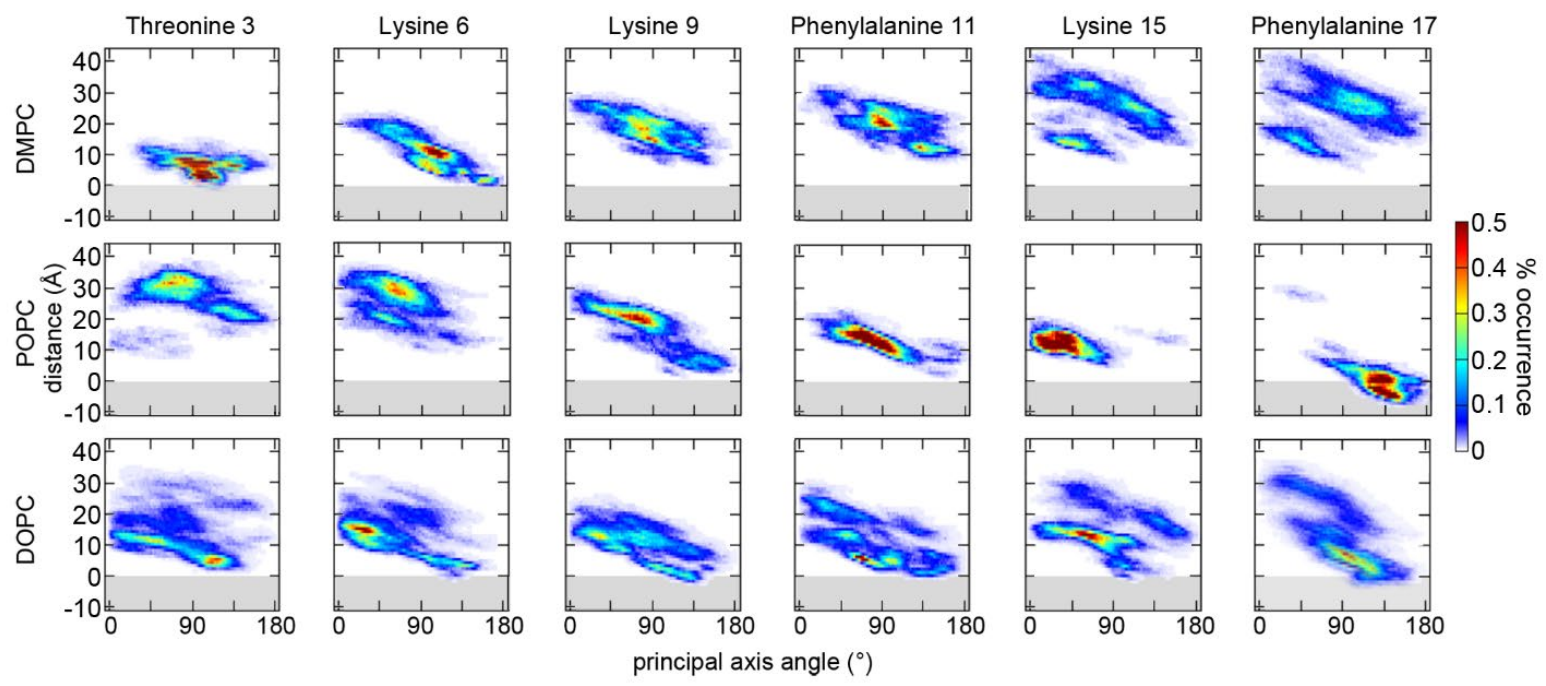

Figure 5.12 Nt17 binding is facilitated via partitioning of amino acid sidechains into bilayer systems with higher areas per lipid. Correlation plot between the binned distribution of sidechain angles of select residues in $\mathrm{Nt17}$ and the binned distribution of the z-distance between each respective residue's terminal atom and the plane of the $\mathrm{P}$ atoms in the lipid headgroups. ( 0 deg: parallel to bilayer normal, 90 deg: parallel to bilayer surface; 180 deg: antiparallel to bilayer normal (i.e., pointing into the bilayer)). Top row: DMPC; middle row: POPC; bottom row: DOPC. 


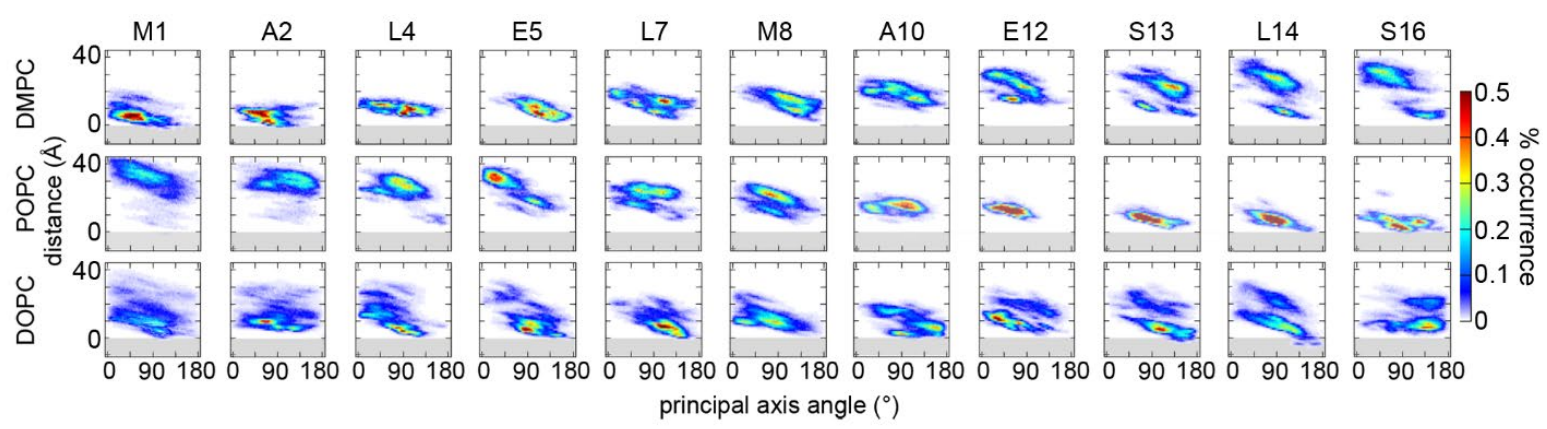

Figure 5.13 Lipid tails influence the partitioning of $\mathrm{Nt17}$ amino acid sidechains into the bilayer. Correlation plot between the binned distribution of sidechain angles of select residues in $\mathrm{Nt} 17$ and the binned distribution of the z-distance between each respective residue's terminal atom and the plane of the $\mathrm{P}$ atoms in the lipid headgroups. ( 0 deg: parallel to bilayer normal, $90 \mathrm{deg}$ : parallel to bilayer surface; $180 \mathrm{deg}$ : antiparallel to bilayer normal (i.e., pointing into the bilayer)). Top row: DMPC; middle row: POPC; bottom row: DOPC.

\subsubsection{The role of protrusions and defects in binding and stabilization of Nt17}

When examining the behavior of the bilayer surface in the upper leaflet, each lipid system possesses areas of protrusions and defects. In general, areas of protrusions or of defects tend to group together (Fig. 5.14a). POPC has more well-defined and broader areas of defects compared to DMPC, which correlates with the more effective binding of $\mathrm{Nt} 17$. DOPC had the fewest areas of protrusions and defects, likely contributing to the global but weaker interactions of Nt17. Quantification of the total number of protrusions and defects in the bilayer surface provides a more well-defined description of what occurs when Nt17 binds to a membrane. In the absence of $\mathrm{Nt17}$, the three lipid systems have similar distributions of protrusions ("above", Fig. 5.14b) but slightly different distribution of defects (“below”, Fig. 5.14b). Upon Nt17 binding, two changes take place in the bilayer surface: 1) the population of protrusions and defects decreases due to partitioning of the peptide, and 2) the distribution of protrusions and of defects between lipid systems becomes separable. With protrusions, the distributions remain consistent across all lipid systems; with defects, the distributions arrange according to the corresponding 
areas per lipid. The difference in the shifts in the distribution of protrusions indicates that the initial interactions of $\mathrm{Nt} 17$ with the bilayer surface (i.e., with protrusions) is entirely non-specific with respect to lipid type. In contrast, the number and distribution of defects is most greatly affected by the ability of the bilayer to accommodate partitioning of Nt17 into the headgroup region. Since DMPC is the most tightly-packed bilayer in the lateral plane of the membrane, both by area per lipid and by the number of defects, it is incapable of easily changing its lateral compressibility, a necessary prerequisite for surface binding and partitioning of a peptide. This underscores why POPC and DOPC have progressively larger distributions of defects.
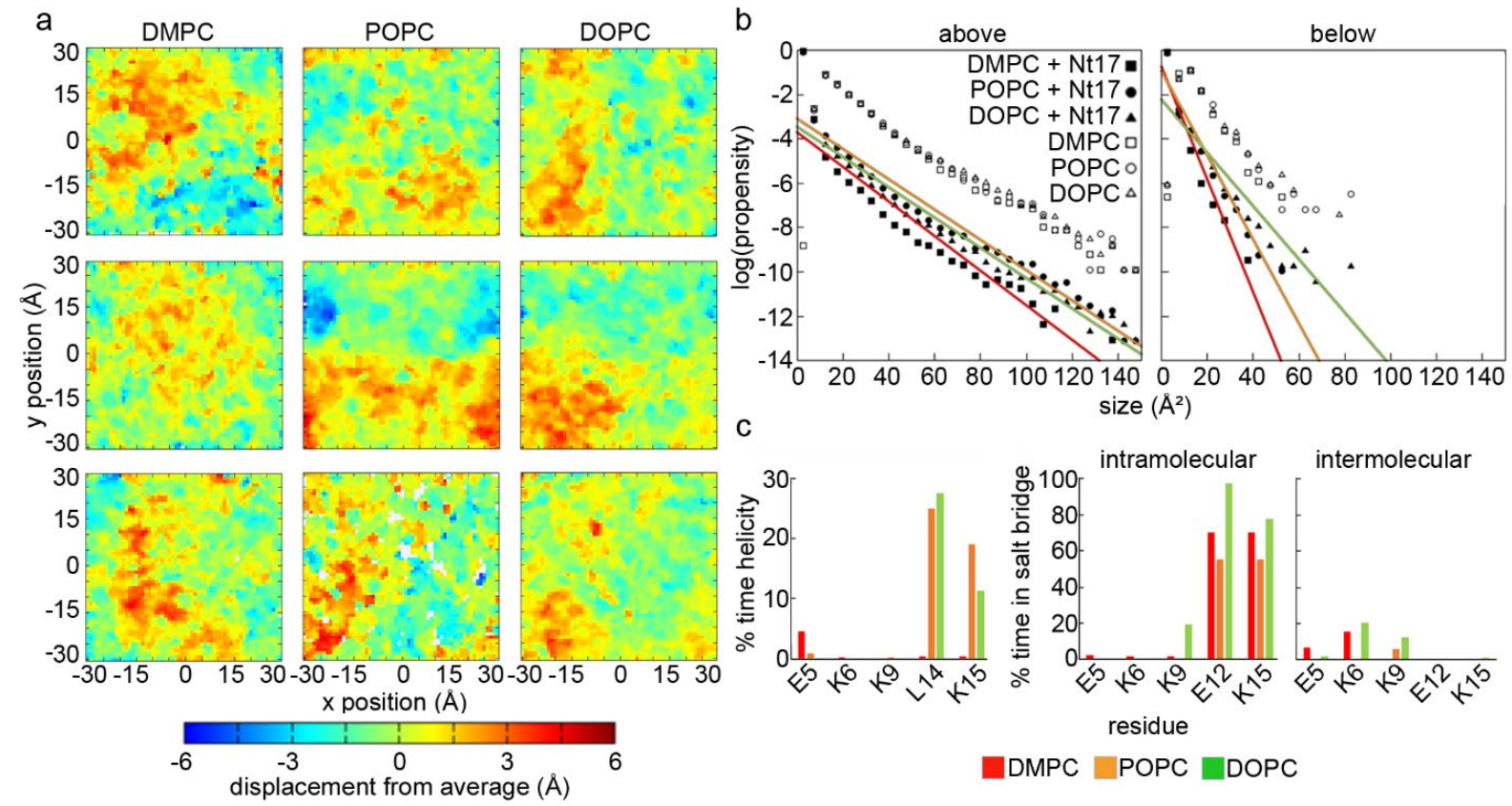

Figure 5.14 Formation of stable proteolipid complexes occurs via contributions from both redistribution of bilayer deformations and stable intermediates of Nt17. a) Heat map of the average displacement of headgroups in the upper leaflet for all three trajectories of DMPC (left), POPC (middle), and DOPC (right) as measured with membrane_map in LOOS. b) Logarithmic distribution of protrusions ("above", left) and defects ("below", right) as calculated with the measure SASA tool in VMD. c) Percent helicity for Nt17 in DMPC, POPC, and DOPC. (Residues that were not helical were omitted for clarity.) d) Percent of total simulation time a charged residue in $\mathrm{Nt} 17$ was involved in formation of a salt bridge for DMPC, POPC, and DOPC. 
Non-bonded interactions also play a role in stable binding of Nt17 to the membrane. Although we did not observe the extensive formation of an $\alpha$-helix that was reported by Bechinger and coworkers, ${ }^{24} \mathrm{Nt} 17$ undergoes partial helical folding in POPC and DOPC (Fig. 5.14c). Folding is centered around L14 and K15, with about 20-25\% helicity. Additionally, an intramolecular salt bridge frequently exists between E12 and K15. i +3 salt bridges can contribute to $\alpha$-helix formation in short peptides; ${ }^{70}$ in this case, the salt bridge formation appears to work cooperatively with headgroup accessibility in facilitating Nt17 partitioning into the bilayer.

\subsection{Discussion}

A number of cytotoxic features of HD are associated with the localization of htt to lipid membranes. ${ }^{38-40}$ In this study, the impact of lipid tail saturation on htt-exon1(46Q) interaction with lipid membranes and subsequent consequences on aggregation were determined. The different lipids influenced htt aggregation to varying extents, with DMPC accelerating fibrillization, POPC having a minimal impact, and DOPC decreasing fibrillization. However, the extent of htt association with each lipid system did not correlate with the extent of fibrillization, indicating that the membrane environment provided by different lipid systems influences the membrane activity and aggregation of htt. In addition, the interaction of the htt-exon1 lipid binding domain, i.e. Nt17, with the lipid systems demonstrated that the match between defect and hydrophobic amino acid size determined the orientation of the peptide on the bilayer. These early interactions between Nt17 and each lipid system manifest in down-stream impacts on httexon1(46Q) aggregation. 
Components within the cellular environment, particularly lipid membranes, modify amyloid formation, promoting or stabilizing specific aggregate species. ${ }^{71} \mathrm{Htt}$ undergoes unique aggregation mechanisms on lipid membranes compared to bulk solution, ${ }^{41}$ and this appears dependent on membrane composition. ${ }^{46,47,72}$ Beyond the three simple lipid systems investigated here, more complicated lipid systems also modify htt aggregation to various extents. ${ }^{45,47,73,74}$ Total brain lipid extract (TBLE) stabilizes oligomers of truncated polyQ peptides that contain $\mathrm{Nt} 17^{22}$ and impedes fibrillization of htt-exon $1,{ }^{45}$ and this impact is further modified by the exogenous addition of cholesterol, ${ }^{47}$ sphingomyelin, and GM1 ${ }^{46}$ In contrast, htt fibrillization is accelerated in the presence of POPC/POPS through a unique Nt17-mediated mechanism involving membrane anchoring and two-dimensional diffusion. ${ }^{41}$ Based on 3D HNCO NMR studies using a small peptide with Nt17 and a polyQ domain of 17 residues, the interaction of Nt17 with lipid membranes is dynamic; however, there were clear differences in the interaction of Nt17 with DOPE/DOPS/DOPC compared to brain extracts. ${ }^{72}$ In this regard, the varied orientations of Nt17 observed here by MD on different lipid systems impact htt anchoring and diffusion along the membrane. These initial changes in Nt17-lipid binding manifested in altered abundances of Nt17/lipid complexes and lead to altered aggregation. Collectively, these observations indicate that the initial htt/lipid interactions profoundly impact subsequent aggregation.

While the extent of htt fibrillization correlated with lipid tail saturation (DMPC $>$ POPC > DOPC; determined by ThT and AFM assays), the htt-membrane association (POPC > DMPC > DOPC; measured by PDA and MS) did not, suggesting a more complex relationship between htt-lipid interaction and subsequent aggregation. Through MD simulations, clear differences occur in the initial Nt17-lipid interactions that likely influence downstream aggregation. While a 
significant gap in timescale persists, Nt17/lipid contact density measured in MD simulations are consistent with the populations of Nt17/lipid complexes observed by MS with all three lipids. As Nt17 peptides did not contain polyQ tracts, the MD and MS studies cannot provide insight into fibrillization; however, oligomerization is driven by Nt17..$^{25,43,44,75}$ Therefore, changes in Nt17lipid interaction should impact downstream htt oligomerization, and all three lipid systems reduced the heterogeneity of oligomer populations compared to aggregation in the absence of lipids. This impact of lipids on htt aggregation is significant as cellular dysfunction and toxicity are linked to both htt oligomers ${ }^{12,14}$ and fibrils. ${ }^{19,20}$ Furthermore, the formation or stabilization of specific aggregate species by lipid membranes could promote the toxic disruption of membranes and organelles observed in HD..$^{30,76,77}$ This suggests that targeting the initial htt/lipid interaction may represent an upstream therapeutic target.

The properties determining selectivity of AHs (like Nt17) for specific membranes are often related to curvature-induced membrane defects. ${ }^{49,78,79}$ As curvature enhances the membrane binding of $\mathrm{Nt17},,^{80,81}$ defect sensing represents a plausible mechanism associated with htt membrane affinity. Here, the population of available membrane defects correlates well with the affinity of Nt17 for each lipid system, and the orientation of Nt17 on membranes appears to be predicated by matching residue and defect sizes. Such a scenario has been observed with other AH lipid binding motifs. For example, the amphipathic lipid-packing sensor (ALPS) inserts large hydrophobic residues preferentially into membranes with larger membrane defects. ${ }^{82} \mathrm{Nt} 17$ exhibits many similarities with ALPS motifs, including a structural transition from intrinsically disordered to an $\mathrm{AH}$ in the presence of lipids, ${ }^{23,24,83}$ increased association with membranes of lower cholesterol content ${ }^{47,52}$ or increased curvature ${ }^{78,80}$ and, as shown here, a preference for insertion into bilayers comprised of monounsaturated phospholipids. ${ }^{52,84}$ Despite sharing 
functional similarities, Nt17 and ALPS motifs vary in sequence; while ALPS motifs contain a hydrophobic side comprised primarily of large amino acids (F, L, W), ${ }^{49,82}$ the hydrophobic face of Nt17 contains several smaller amino acids (A, T) near the N-terminus. The lack of variety in ALPS motifs hydrophobic residue sizes leads to the initial insertion of hydrophobic residues into the bilayer in a random manner. ${ }^{48}$ The mechanism of Nt17 membrane binding appears more complex, with both hydrophobic residues size and defect size determining the initial orientation and selective binding of the motif. This defect mechanism gains further importance for selective binding of AHs taken lipid composition and curvature synergistically determine defect size and distribution. ${ }^{85}$

Upon exposure to tightly packed DMPC (saturated tails) bilayers with a relatively small area per lipid, the smaller, N-terminal residues (MATL) partitioned into the bilayer. POPC (monounsaturated) bilayers display an increased area per lipid and occurrence of defects. The interaction between $\mathrm{Nt} 17$ and the POPC bilayer was driven by the partitioning of the larger, Cterminal residues (LKSF). While the smaller N-terminal residues could insert into the larger defects present on POPC bilayers, Nt17 preferred a C-terminus approach, indicating a thermodynamic advantage to sequestering larger hydrophobic residues. DOPC (polyunsaturated) bilayers had a higher propensity to form defects with a broader distribution of sizes. Due to this, residues along the entire length of Nt17 exhibited more frequent, but weaker binding and Nt17 sampled a broader variety of orientations on the bilayer surface. The interaction between Nt17 and DOPC is nonspecific and weak, explaining why complexes observed with MS had larger DOPC content but were significantly less abundant overall than complexes formed with DMPC or POPC. 
Beyond the impact of lipids on htt aggregation, htt and its aggregate forms can induce physical changes in membranes. Many amyloid-forming proteins have deleterious impact on membrane integrity that leads to dysfunction, organelle disruption, and cell death by specifically targeting bilayers. ${ }^{30,71,77}$ For example, A $\beta$, IAPP, and htt all distort membrane morphology and rigidity of model lipid bilayers. ${ }^{86}$ Specifically, mutant htt is associated with membrane degradation of the ER, nuclear envelope, and mitochondria. ${ }^{38-40}$ With the complex nature of amyloid formation, assigning specific aggregate species to a particular toxic mechanism is challenging. This is further complicated by the dynamic nature of aggregation with the process of aggregation potentially playing a role rather than a specific aggregate. That is, understanding the link between the initial protein/lipid interaction, subsequent aggregation, and impact on membrane integrity would provide a clearer, complete picture of these phenomena.

\subsection{References}

1. Penney, J. B., Vonsattel, J. P., MacDonald, M. E., Gusella, J. F. \& Myers, R. H. CAG repeat number governs the development rate of pathology in Huntington's disease. Ann. Neurol. 41, 689-692 (1997).

2. Snell, R. G. et al. Relationship between trinucleotide repeat expansion and phenotypic variation in Huntington's disease. Nat. Gen. 4, 393-397 (1993).

3. Kar, K., Jayaraman, M., Sahoo, B., Kodali, R. \& Wetzel, R. Critical nucleus size for disease-related polyglutamine aggregation is repeat length dependent. Nat. Struct. Mol. Biol. 18, 328-336 (2011).

4. Lotz, G. P. \& Legleiter, J. The role of amyloidogenic protein oligomerization in neurodegenerative disease. J. Mol. Med. 91, 653-664 (2013).

5. Bonfanti, S. et al. Molecular mechanisms of heterogeneous oligomerization of huntingtin proteins. Sci. Rep. 9, 7615 (2019).

6. Arndt, J. R. et al. Huntingtin N-terminal monomeric and multimeric structures destabilized by covalent modification of heteroatomic residues. Biochemistry 54, 4285-4296 (2015).

7. Burke, K. A., Godbey, J. \& Legleiter, J. Assessing mutant huntingtin fragment and polyglutamine aggregation by atomic force microscopy. Methods 53, 275-284 (2011).

8. Muchowski, P. J. et al. Hsp70 and Hsp40 chaperones can inhibit self-assembly of polyglutamine proteins into amyloid-like fibrils. Proc. Natl. Acad. Sci. U.S.A. 97, 7841-7846 (2000). 
9. Counterman, A. E., Hilderbrand, A. E., Barnes, C. A. S. \& Clemmer, D. E. Formation of peptide aggregates during ESI: Size, charge, composition, and contributions to noise. J. Am. Soc. Spectrom. 12, 1020-1035 (2001).

10. DiFiglia, M. et al. Aggregation of huntingtin in neuronal intranuclear inclusions and dystrophic neurites in brain. Science 277, 1990-1993 (1997).

11. Arrasate, M., Mitra, S., Schweitzer, E. S., Segal, M. R. \& Finkbeiner, S. Inclusion body formation reduces levels of mutant huntingtin and the risk of neuronal death. Nature 431, 805-810 (2004).

12. Kim, Y. E. et al. Soluble Oligomers of PolyQ-Expanded Huntingtin Target a Multiplicity of Key Cellular Factors. Mol. Cell 63, 951-964 (2016).

13. Olshina, M. A. et al. Tracking mutant huntingtin aggregation kinetics in cells reveals three major populations that include an invariant oligomer pool. J. Biol. Chem. 285, 21807-21816 (2010).

14. Lajoie, P. \& Snapp, E. L. Formation and toxicity of soluble polyglutamine oligomers in living cells. PLoS ONE 5, e15245 (2010).

15. Legleiter, J. et al. Mutant Huntingtin Fragments Form Oligomers in a Polyglutamine Lengthdependent Manner in Vitro and in Vivo. J. Biol. Chem. 285, 14777-14790 (2010).

16. Sahl, S. J., Weiss, L. E., Duim, W. C., Frydman, J. \& Moerner, W. E. Cellular Inclusion Bodies of Mutant Huntingtin Exon 1 Obscure Small Fibrillar Aggregate Species. Sci. Rep. 2, 1-7 (2012).

17. Lu, M. et al. Live-cell super-resolution microscopy reveals a primary role for diffusion in polyglutamine-driven aggresome assembly. J. Biol. Chem. 294, 257-268 (2019).

18. Nucifora, L. G. et al. Identification of novel potentially toxic oligomers formed in vitro from mammalian-derived expanded huntingtin exon-1 protein. J. Biol. Chem. 287, 16017-16028 (2012).

19. Drombosky, K. W. et al. Mutational analysis implicates the amyloid fibril as the toxic entity in Huntington's disease. Neurobiol. Dis. 120, 126-138 (2018).

20. Pieri, L., Madiona, K., Bousset, L. \& Melki, R. Fibrillar $\alpha$-Synuclein and Huntingtin Exon 1 Assemblies Are Toxic to the Cells. Biophys. J. 102, 2894-2905 (2012).

21. Bäuerlein, F. J. B. et al. In Situ Architecture and Cellular Interactions of PolyQ Inclusions. Cell 171, 179-187.e10 (2017).

22. Burke, K. A., Kauffman, K. J., Umbaugh, C. S., Frey, S. L. \& Legleiter, J. The Interaction of Polyglutamine Peptides with Lipid Membranes Is Regulated by Flanking Sequences Associated with Huntingtin. J. Biol. Chem. 288, 14993-15005 (2013).

23. Michalek, M., Salnikov, E. S., Werten, S. \& Bechinger, B. Membrane Interactions of the Amphipathic Amino Terminus of Huntingtin. Biochemistry 52, 847-858 (2013). 
24. Michalek, M., Salnikov, E. S. \& Bechinger, B. Structure and Topology of the Huntingtin 1-17 Membrane Anchor by a Combined Solution and Solid-State NMR Approach. Biophys. J. 105, 699-710 (2013).

25. Sivanandam, V. N. et al. The aggregation-enhancing huntingtin N-terminus is helical in amyloid fibrils. J. Am. Chem. Soc. 133, 4558-4566 (2011).

26. Truant, R., Atwal, R. \& Burtnik, A. Hypothesis: Huntingtin may function in membrane association and vesicular trafficking. Biochem. Cell Biol. 84, 912-917 (2006).

27. Velier, J. et al. Wild-Type and Mutant Huntingtins Function in Vesicle Trafficking in the Secretory and Endocytic Pathways. Exp. Neurol. 152, 34-40 (1998).

28. Kegel, K. B. et al. Huntingtin Associates with Acidic Phospholipids at the Plasma Membrane. J. Biol. Chem. 280, 36464-36473 (2005).

29. Burke, K. A., Kauffman, K. J., Umbaugh, C. S., Frey, S. L. \& Legleiter, J. The Interaction of Polyglutamine Peptides with Lipid Membranes Is Regulated by Flanking Sequences Associated with Huntingtin. J. Biol. Chem. 288, 14993-15005 (2013).30. Suopanki, J. et al. Interaction of huntingtin fragments with brain membranes - clues to early dysfunction in Huntington's disease. Journal of Neurochemistry 96, 870-884 (2006).

31. Burke, K. A., Hensal, K. M., Umbaugh, C. S., Chaibva, M. \& Legleiter, J. Huntingtin disrupts lipid bilayers in a polyQ-length dependent manner. Biochim. Biophys. Acta, Biomem. 1828, 1953-1961 (2013).

32. Qin, Z.-H. et al. Huntingtin bodies sequester vesicle-associated proteins by a polyproline-dependent interaction. J. Neurosci. 24, 269-281 (2004).

33. Kegel, K. B. et al. Huntingtin expression stimulates endosomal-lysosomal activity, endosome tubulation, and autophagy. J. Neurosci. 20, 7268-7278 (2000).

34. Valencia, A. et al. Mutant huntingtin and glycogen synthase kinase 3-beta accumulate in neuronal lipid rafts of a presymptomatic knock-in mouse model of Huntington's disease. J. Neurosci. Res. 88, 179-190 (2010).

35. Ruan, Q., Lesort, M., MacDonald, M. E. \& Johnson, G. V. W. Striatal cells from mutant huntingtin knock-in mice are selectively vulnerable to mitochondrial complex II inhibitor-induced cell death through a non-apoptotic pathway. Hum. Mol. Genet. 13, 669-681 (2004).

36. Shirendeb, U. P. et al. Mutant huntingtin's interaction with mitochondrial protein Drp1 impairs mitochondrial biogenesis and causes defective axonal transport and synaptic degeneration in Huntington's disease. Hum. Mol. Genet. 21, 406-420 (2012).

37. Chang, D. T. W., Rintoul, G. L., Pandipati, S. \& Reynolds, I. J. Mutant huntingtin aggregates impair mitochondrial movement and trafficking in cortical neurons. Neurobiol. Dis. 22, 388-400 (2006).

38. Jiang, Y., Chadwick, S. R. \& Lajoie, P. Endoplasmic reticulum stress: The cause and solution to Huntington's disease? Brain Research 1648, 650-657 (2016). 
39. Ueda, M. et al. Expanded polyglutamine embedded in the endoplasmic reticulum causes membrane distortion and coincides with Bax insertion. Biochem. Biophys. Res. Commun. 474, 259-263 (2016).

40. Liu, K.-Y. et al. Disruption of the nuclear membrane by perinuclear inclusions of mutant huntingtin causes cell-cycle re-entry and striatal cell death in mouse and cell models of Huntington's disease. Hum. Mol. Genet. 24, 1602-1616 (2015).

41. Pandey, N. K. et al. The 17-residue-long $\mathrm{N}$ terminus in huntingtin controls stepwise aggregation in solution and on membranes via different mechanisms. J. Biol. Chem. 293, 2597-2605 (2018).

42. Williamson, T. E., Vitalis, A., Crick, S. L. \& Pappu, R. V. Modulation of polyglutamine conformations and dimer formation by the N-terminus of Huntingtin. J. Mol. Biol. 396, 1295-1309 (2010).

43. Jayaraman, M. et al. Kinetically competing huntingtin aggregation pathways control amyloid polymorphism and properties. Biochemistry 51, 2706-2716 (2012).

44. Jayaraman, M. et al. Slow amyloid nucleation via $\alpha$-helix-rich oligomeric intermediates in short polyglutamine-containing huntingtin fragments. J. Mol. Biol. 415, 881-899 (2012).

45. Beasley, M. et al. Lipid membranes influence the ability of small molecules to inhibit huntingtin fibrillization. Biochemistry (2019).

46. Chaibva, M. et al. Sphingomyelin and GM1 Influence Huntingtin Binding to, Disruption of, and Aggregation on Lipid Membranes. ACS Omega 3, 273-285 (2018).

47. Gao, X. et al. Cholesterol Modifies Huntingtin Binding to, Disruption of, and Aggregation on Lipid Membranes. Biochemistry 55, 92-102 (2016).

48. Giménez-Andrés, M., Čopič, A. \& Antonny, B. The Many Faces of Amphipathic Helices. Biomolecules 8, (2018).

49. Drin, G. et al. A general amphipathic alpha-helical motif for sensing membrane curvature. Nat. Struct. Mol. Biol. 14, 138-146 (2007).

50. Masuda, M. et al. Endophilin BAR domain drives membrane curvature by two newly identified structure-based mechanisms. EMBO J 25, 2889-2897 (2006).

51. Doucet, C. M., Talamas, J. A. \& Hetzer, M. W. Cell Cycle Dependent Differences in Nuclear Pore Complex Assembly in Metazoa. Cell 141, 1030-1041 (2010).

52. Pranke, I. M. et al. $\alpha$-Synuclein and ALPS motifs are membrane curvature sensors whose contrasting chemistry mediates selective vesicle binding. J. Cell Biol. 194, 89-103 (2011).

53. Thompson, L. M. et al. IKK phosphorylates Huntingtin and targets it for degradation by the proteasome and lysosome. J. Cell Biol. 187, 1083-1099 (2009).

54. Burke, K. A. \& Legleiter, J. Atomic Force Microscopy Assays for Evaluating Polyglutamine Aggregation in Solution and on Surfaces. in Tandem Repeats in Genes, Proteins, and Disease: Methods and 
Protocols (eds. Hatters, D. M. \& Hannan, A. J.) 21-40 (Humana Press, 2013). doi:10.1007/978-162703-438-8_2.

55. Sokolovski, M., Sheynis, T., Kolusheva, S. \& Jelinek, R. Membrane interactions and lipid binding of casein oligomers and early aggregates. Biochim. Biophys. Acta, Biomem. 1778, 2341-2349 (2008).

56. Zheng, F., Wu, Z. \& Chen, Y. A quantitative method for the measurement of membrane affinity by polydiacetylene-based colorimetric assay. Anal. Biochem. 420, 171-176 (2012).

57. Kew, S. J. \& Hall, E. A. H. pH response of carboxy-terminated colorimetric polydiacetylene vesicles. Anal. Chem. 78, 2231-2238 (2006).

58. Song, J., Cheng, Q., Kopta, S. \& Stevens, R. C. Modulating Artificial Membrane Morphology: pHInduced Chromatic Transition and Nanostructural Transformation of a Bolaamphiphilic Conjugated Polymer from Blue Helical Ribbons to Red Nanofibers. J. Am. Chem. Soc. 123, 3205-3213 (2001).

59. Karanji, A. K. et al. Investigating the interactions of the first 17 amino acid residues of Huntingtin with lipid vesicles using mass spectrometry and molecular dynamics. J. Mass Spectrom. 55, e4470 (2020).

60. Humphrey, W., Dalke, A. \& Schulten, K. VMD: Visual molecular dynamics. J. Mol. Graph. 14, 33-38 (1996).

61. Phillips, J. C. et al. Scalable molecular dynamics with NAMD. J. Comput. Chem. 26, 1781-1802 (2005).

62. Romo, T. D. \& Grossfield, A. LOOS: An extensible platform for the structural analysis of simulations. in 2009 Annual International Conference of the IEEE Engineering in Medicine and Biology Society 2332-2335 (2009). doi:10.1109/IEMBS.2009.5335065.

63. Romo, T. D., Leioatts, N. \& Grossfield, A. Lightweight object oriented structure analysis: Tools for building tools to analyze molecular dynamics simulations. J. Comput. Chem. 35, 2305-2318 (2014).

64. Zwier, M. C. et al. WESTPA: An Interoperable, Highly Scalable Software Package for Weighted Ensemble Simulation and Analysis. J. Chem. Theory Comput. 11, 800-809 (2015).

65. Cui, H., Lyman, E. \& Voth, G. A. Mechanism of Membrane Curvature Sensing by Amphipathic Helix Containing Proteins. Biophys. J. 100, 1271-1279 (2011).

66. Garcia, A. E. \& Sanbonmatsu, K. Y. -Helical stabilization by side chain shielding of backbone hydrogen bonds. Proc. Natl. Acad. Sci. U.S.A. 99, 2782-2787 (2002).

67. Kucerka, N. et al. Structure of fully hydrated fluid phase DMPC and DLPC lipid bilayers using X-ray scattering from oriented multilamellar arrays and from unilamellar vesicles. Biophys. J. 88, 26262637 (2005).

68. Kucerka, N., Tristram-Nagle, S. \& Nagle, J. F. Structure of fully hydrated fluid phase lipid bilayers with monounsaturated chains. J. Membr. Biol. 208, 193-202 (2005). 
69. Tristram-Nagle, S., Petrache, H. I. \& Nagle, J. F. Structure and interactions of fully hydrated dioleoylphosphatidylcholine bilayers. Biophys. J. 75, 917-925 (1998).

70. Marqusee, S. \& Baldwin, R. L. Helix stabilization by Glu-...Lys + salt bridges in short peptides of de novo design. Proc. Natl. Acad. Sci. U.S.A. 84, 8898-8902 (1987).

71. Burke, K. A., Yates, E. A. \& Legleiter, J. Biophysical Insights into How Surfaces, Including Lipid Membranes, Modulate Protein Aggregation Related to Neurodegeneration. Front. Neurol. 4, (2013).

72. Levy, G. R. et al. Huntingtin's N-Terminus Rearrangements in the Presence of Membranes: A Joint Spectroscopic and Computational Perspective. ACS Chem. Neurosci. 10, 472-481 (2019).

73. Zhang, J., Tan, J., Pei, R. \& Ye, S. Acidic Environment Significantly Alters Aggregation Pathway of Human Islet Amyloid Polypeptide at Negative Lipid Membrane. Langmuir 36, 1530-1537 (2020).

74. Hane, F., Drolle, E., Gaikwad, R., Faught, E. \& Leonenko, Z. Amyloid- $\beta$ aggregation on model lipid membranes: an atomic force microscopy study. J. Alzheimers Dis. 26, 485-494 (2011).

75. Mishra, R. et al. Inhibiting the nucleation of amyloid structure in a huntingtin fragment by targeting $\alpha$-helix-rich oligomeric intermediates. J. Mol. Biol. 415, 900-917 (2012).

76. Choo, Y. S., Johnson, G. V. W., MacDonald, M., Detloff, P. J. \& Lesort, M. Mutant huntingtin directly increases susceptibility of mitochondria to the calcium-induced permeability transition and cytochrome c release. Hum. Mol. Genet. 13, 1407-1420 (2004).

77. Eckmann, J. et al. Mitochondrial Membrane Fluidity is Consistently Increased in Different Models of Huntington Disease: Restorative Effects of Olesoxime. Mol. Neurobiol. 50, 107-118 (2014).

78. Drin, G. \& Antonny, B. Amphipathic helices and membrane curvature. FEBS Lett. 584, 1840-1847 (2010).

79. Hatzakis, N. S. et al. How curved membranes recruit amphipathic helices and protein anchoring motifs. Nat. Chem. Biol. 5, 835-841 (2009).

80. Chaibva, M., Burke, K. A. \& Legleiter, J. Curvature Enhances Binding and Aggregation of Huntingtin at Lipid Membranes. Biochemistry 53, 2355-2365 (2014).

81. Tao, M., Pandey, N. K., Barnes, R., Han, S. \& Langen, R. Structure of Membrane-Bound Huntingtin Exon 1 Reveals Membrane Interaction and Aggregation Mechanisms. Structure 27, 1570-1580.e4 (2019).

82. Vanni, S. et al. Amphipathic Lipid Packing Sensor Motifs: Probing Bilayer Defects with Hydrophobic Residues. Biophys. J. 104, 575-584 (2013).

83. Prévost, C. et al. Mechanism and Determinants of Amphipathic Helix-Containing Protein Targeting to Lipid Droplets. Dev. Cell 44, 73-86.e4 (2018).

84. van Meer, G., Voelker, D. R. \& Feigenson, G. W. Membrane lipids: where they are and how they behave. Nat. Rev. Mol. Cell Biol. 9, 112-124 (2008). 
85. Vanni, S., Hirose, H., Barelli, H., Antonny, B. \& Gautier, R. A sub-nanometre view of how membrane curvature and composition modulate lipid packing and protein recruitment. Nat. Comm. 5, 4916 (2014).

86. Burke, K. A., Yates, E. A. \& Legleiter, J. Amyloid-Forming Proteins Alter the Local Mechanical Properties of Lipid Membranes. Biochemistry 52, 808-817 (2013). 


\section{Conclusion and Future Directions}

\subsection{Introduction}

Huntington's Disease (HD) is a fatal neurodegenerative disorder that is caused by an expanded glutamine repeat (polyQ) domain of htt. As a result of the expanded polyQ domain, mutant htt misfolds into a variety of toxic aggregate species including oligomers, annular aggregates, and fibrils. The interaction of pathogenic htt monomers and aggregates with the membranes of subcellular organelles results in a variety of toxic mechanisms that encourage the progression of HD. ${ }^{1-4}$ Currently, the details of the toxic mechanisms associated with the interaction of htt aggregates with lipid membranes are not well understood. Understanding the mechanism of htt association with lipid membranes increases the understanding of membraneassociated toxic mechanisms of htt and presents targets for HD therapeutic strategies.

In order to understand the factors influencing the interactions between htt and lipid membranes, it is critical to develop techniques that can be used to efficiently measure membrane association. Polydiacetylene (PDA) lipid binding assays have emerged as a simple, cost-effective

method of measuring membrane affinity with the potential for high-throughput application, ${ }^{5,6}$ but the effect of lipid composition on membrane fluidity and assay response makes the quantitative comparison of different PDA/lipid systems challenging. In Chapter 2, we aimed to develop a PDA-based assay to directly compare the interactions between molecules and vesicles of varying lipid composition (Fig. 6.1). This study demonstrated that exposing PDA/lipid vesicles with DOPC, POPC, POPE, or POPG as the lipid component to increasing concentrations of $\mathrm{NaOH}$ resulted in a linear increase in \% CR until the $45 \mathrm{mM}$ dose, at which point increasing the $\mathrm{NaOH}$ concentration no longer resulted in further colorimetric shift. Despite the linear response of \% 
$\mathrm{CR}$ to $\mathrm{NaOH}$ exposure within the working range for all systems, the magnitude of the response from system to system varied greatly. To account for this, the maximum CR for each system was determined by incubation of the PDA/lipid vesicles with a concentration of $\mathrm{NaOH}$ that was well above the working range. By normalizing the assays to the maximum $\mathrm{CR}$ for each lipid system, batch-to-batch and between-lipid RSD values were lowered significantly, allowing for meaningful comparisons of results obtained with different lipid systems.

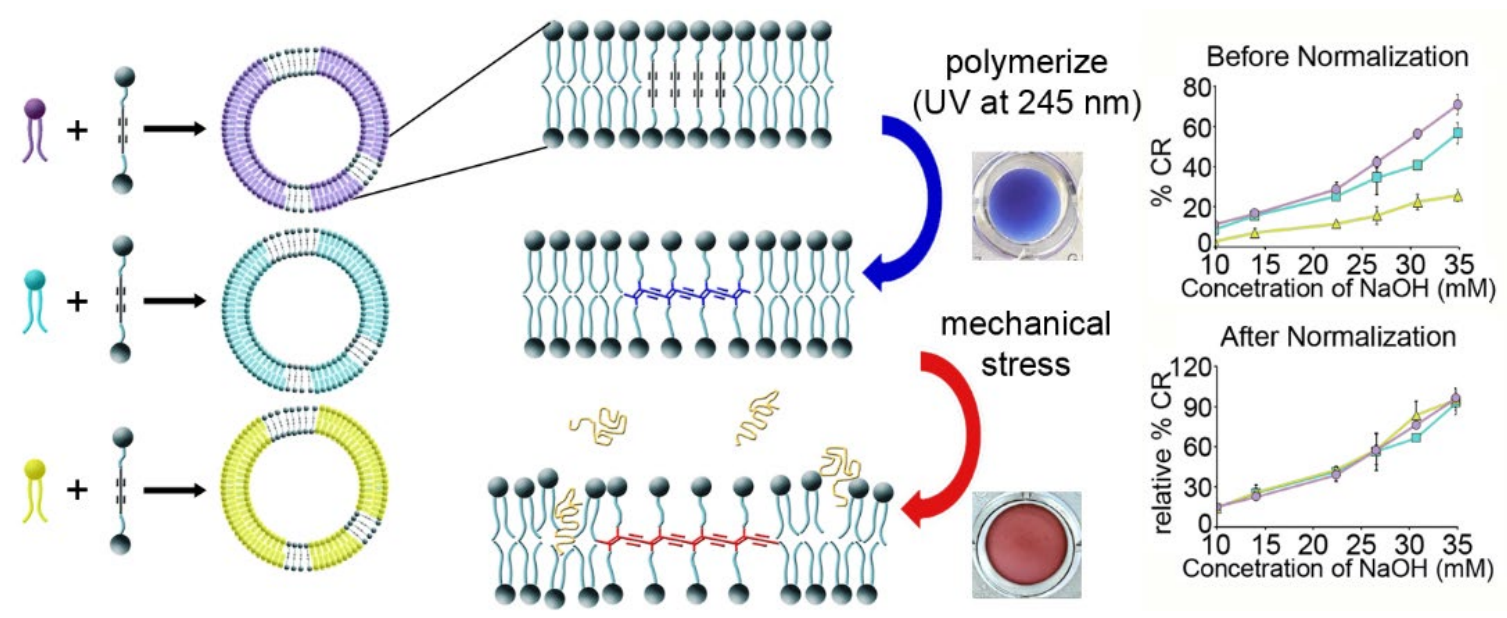

Figure 6.1 A schematic presenting a method for PDA lipid binding assay normalization. PDA/lipid vesicles are formed by mixing the PDA monomers with hydrated lipid and allowing it to self-assemble into vesicles. Once formed, the PDA is polymerized with UV light to result in blue vesicles that undergo a colorimetric shift to red when mechanical stress is applied. The normalization of the PDA assay decreases batch-to-batch variation and variation between lipid systems.

The interaction of htt with lipid membranes alters the mechanism of aggregation and can stabilize specific aggregate species and morphologies. ${ }^{7-9}$ With the formation of aggregate species being a hallmark of $\mathrm{HD},{ }^{2,10}$ studies have attempted to identify small molecules that inhibit amyloid formation. However, with the influence of membranes on htt aggregate mechanisms, the efficiency of these small molecules in inhibiting aggregation may be altered by the presence of lipid membranes. In Chapter 3, we sought to determine if aggregation inhibitors epigallocatechin 
3-gallate (EGCG) and curcumin maintain their efficiency in the presence of a pure lipid system (POPC) and physiologically relevant system (TBLE) (Fig. 6.2). EGCG reduced the interaction of htt with both POPC and TBLE vesicles, and it remained effective in inhibiting fibrillization in both cases. On the other hand, curcumin reduced htt association with both lipid systems but became ineffective in inhibiting fibrillization the presence of POPC vesicles and actually increased fibrillization in the presence of TBLE vesicles. The difference could lie in how the two small molecules act to inhibit fibrillization; EGCG acts to target early events in htt aggregation, ${ }^{11}$ explaining why it remains effective in the presence of lipid vesicles, while curcumin may target later stages of aggregation that are altered by the presence of lipid membranes and thus decrease the ability of curcumin to inhibit aggregation. Collectively, the results of Chapter 3 highlight that htt aggregation and the effectiveness of small molecule aggregation inhibitors are largely influenced by components of the cellular environment like lipid membranes.

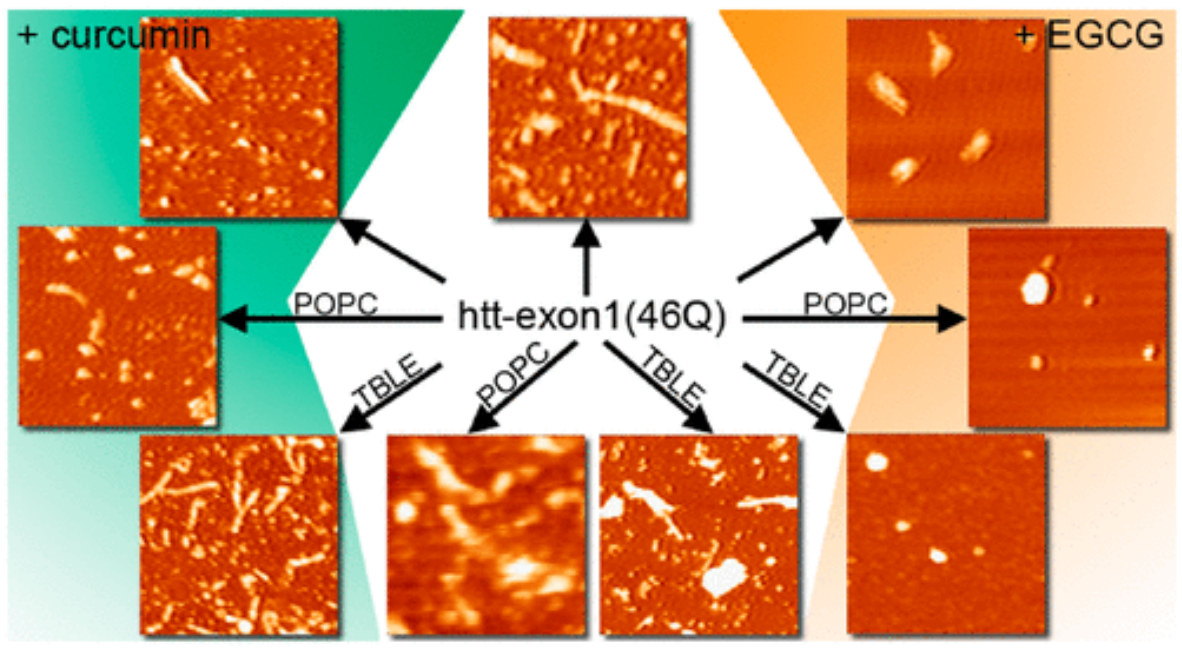

Figure 6.2 Lipid membranes influence the efficiency of small molecules in inhibiting htt aggregation. EGCG is still an efficient htt aggregation inhibitor in the presence of POPC and TBLE lipid vesicles. On the other hand, curcumin is no longer an effective aggregation inhibitor in the presence of POPC vesicles, and cur actually increases aggregation in the presence of TBLE vesicles. 


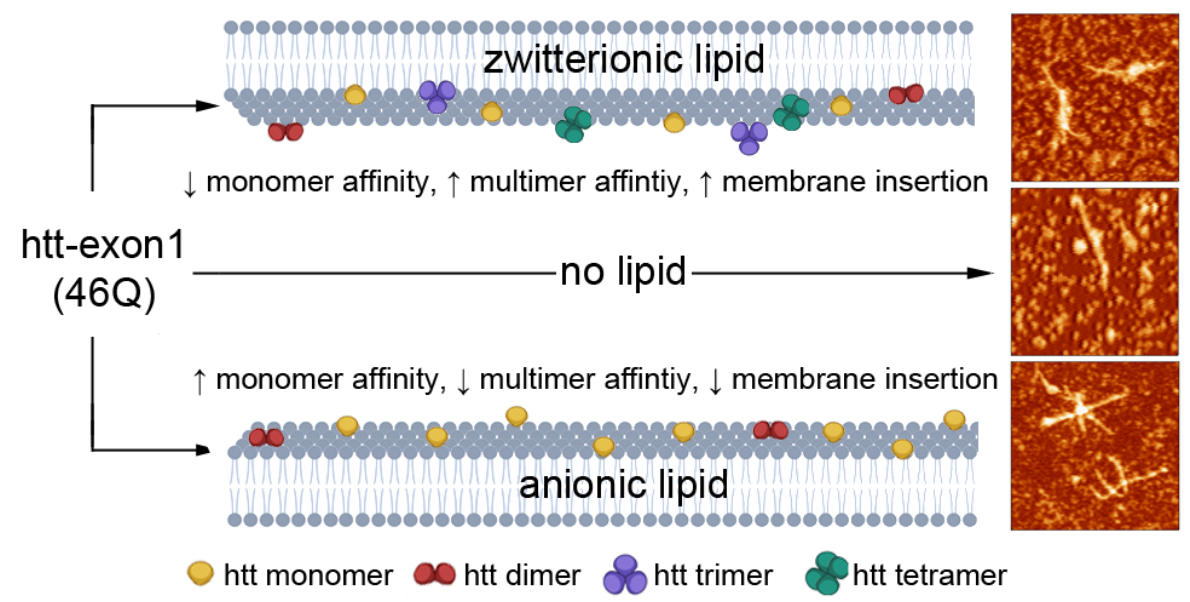

Figure 6.3 The mechanism of htt lipid binding and aggregation are altered by membrane charge. Htt multimers exhibit a higher affinity for zwitterionic lipids, while monomers exhibit a higher affinity for anionic lipids. Also, htt does not appreciably insert into anionic membranes, resulting in a localization of htt at the surface of the membranes that encourages htt aggregation.

Results from Chapter 3 revealed that htt associated with POPC and TBLE vesicles to different extents, indicating that htt affinity for membranes is influenced by the membrane composition. Determining the lipid properties that influence the interaction of htt with specific membranes is important for the understanding of a number of htt normal functions ${ }^{1,12}$ and cytotoxic mechanisms. ${ }^{3,13,14}$ Therefore, in Chapters 4 and 5, we set out to determine how membrane physicochemical properties alter htt-lipid affinity and htt aggregation. Chapter 4 specifically focused on membrane properties associated with the size and charge of the lipid headgroup (Fig. 6.3). PDA assays revealed that htt associated less with negatively charged membranes, and MS analysis on htt-lipid complexes revealed a localization of monomers at the surface of the vesicle. The combined PDA and MS results indicate a barrier between the anchoring and insertion step of $\mathrm{Nt} 17$ when interacting with negatively charged vesicles. As a result, htt localizes at the bilayer surface and increases htt fibrillization as observed by ThT and AFM in the presence of negatively charged lipids. In addition, htt exhibited a higher affinity for 
the most fluid system when comparing membranes of similar charge. In Chapter 5, the focus was on the influence of membrane properties associated with the saturation of the lipid tail on htt aggregation and lipid binding. In this case, the trend of the lipid effect on htt aggregation as observed by ThT and AFM (DMPC $>$ POPC $>$ DOPC) did not match the trend of membrane affinity observed by both PDA and MS (POPC > DMPC > DOPC), indicating a complex combination of mechanisms occurring in the presence of these membranes. Despite the similar charge of the bilayers, MD studies revealed that Nt17 approached each bilayer differently, and residue insertion seemed to be dependent on both residue size and the size of available bilayer defects. Together, the results from Chapters 4 and 5 reveal that membrane fluidity, charge density, and defect size play a role in determining the selectivity of htt affinity for membranes.

The work presented in this dissertation has increased our understanding of the factors contributing to the selective interaction of htt with lipid membranes, but further work must be done to fully understand the mechanisms of interaction and induced toxicity that contribute to the progression of HD. Here, future directions based on the results obtained during these studies will be presented that could further advance the understanding of htt mechanisms with respect to membrane association: 1) the effect of cholesterol on the interaction of htt with membranes of different physicochemical properties; 2) the properties of membranes that determine the selectivity of specific htt aggregate species; and 3) the role of hydrophobic amino acid size in the selective membrane affinity of amphipathic $\alpha$-helices.

\subsection{Research in progress: Elucidating the effect of membrane cholesterol content on htt aggregation and membrane affinity}

Lipid membranes heavily influence the aggregation of htt, but the role that specific lipid components play is not yet fully understood. For example, cholesterol is an important component 
of biological membranes, with $23 \%$ of mammal's total body cholesterol existing in the brain. ${ }^{15}$ Cholesterol is a significant structural component of neuronal membranes ${ }^{16}$ and maintains the integrity of synapses by defining membrane functional properties. ${ }^{17,18}$ By altering bilayer properties such as fluidity, bilayer thickness, and surface hydrophobicity, cholesterol influences crucial functions such as signal transduction, synaptogenesis, and neurotransmitter release. ${ }^{19-24}$ Patients with HD show abnormalities in cholesterol metabolism and homeostasis, but it is debated whether their cholesterol levels decrease ${ }^{25-28}$ or increase. ${ }^{29-31}$ Alterations to cholesterol homeostasis has been linked to altered neuronal ${ }^{32}$ and neurotransmitter receptor function ${ }^{33}$ and leas ads to neurological symptoms in both mice and humans. ${ }^{34}$ Due to the potential importance of cholesterol in HD, a series of preliminary experiments were performed that complement the studies in Chapters 4 and 5. Results from Chapters 4 and 5 indicate that bilayer physicochemical properties play a role in determining htt affinity for membranes, so altered physicochemical properties as a result of altered cholesterol content in membranes likely influences htt-lipid interaction. Pure lipid systems POPC, DOPC, and POPG were spiked with 0, 10, or 20\% cholesterol to determine the effect of increased cholesterol content on htt aggregation and lipid binding relative to the physicochemical properties of the pure lipid system. ThT assays and AFM were used to gather information on aggregation kinetics and morphologies in the presence of each lipid system, and PDA assays were be used to monitor the binding of htt to the lipid vesicles. 

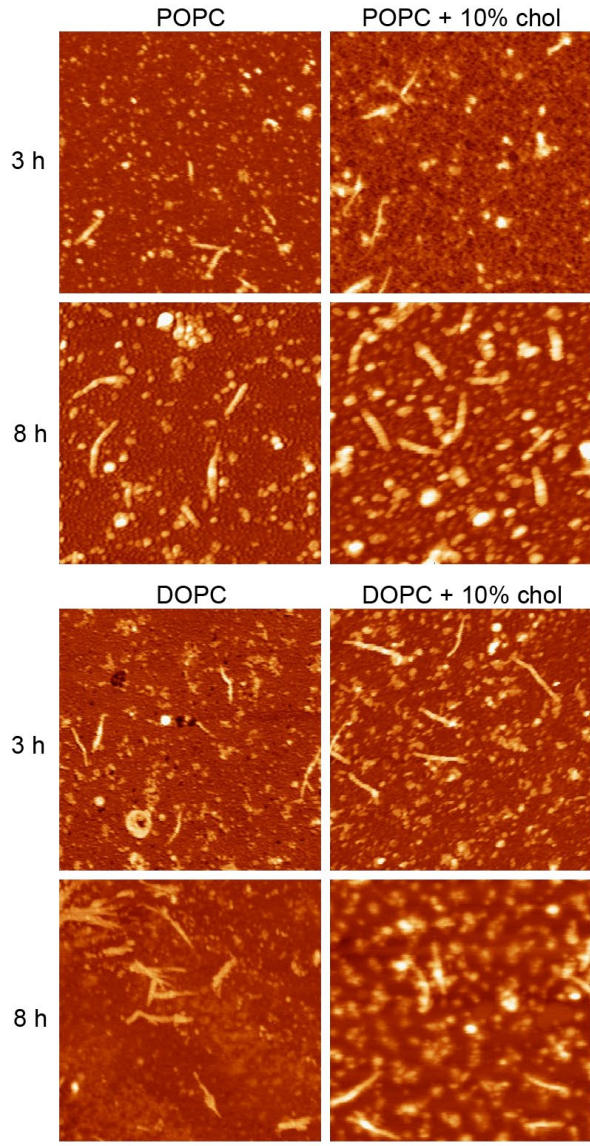

$\mathrm{DOPC}+10 \% \mathrm{chol}$

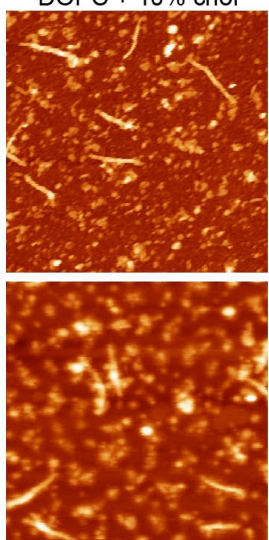

POPG $+10 \%$ chol
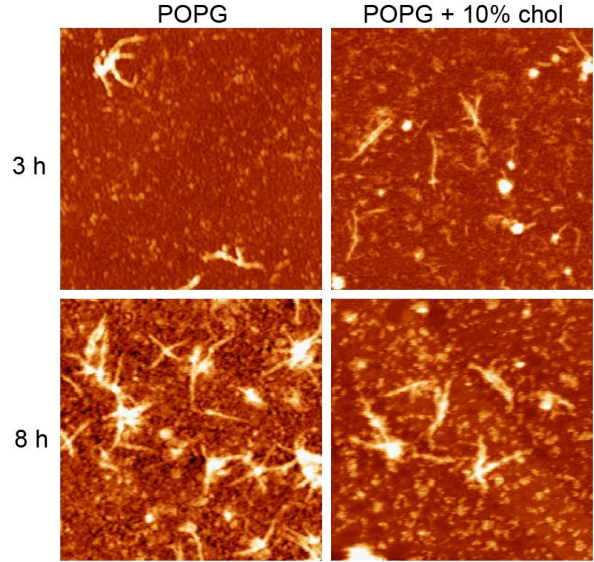

POPC $+20 \%$ chol
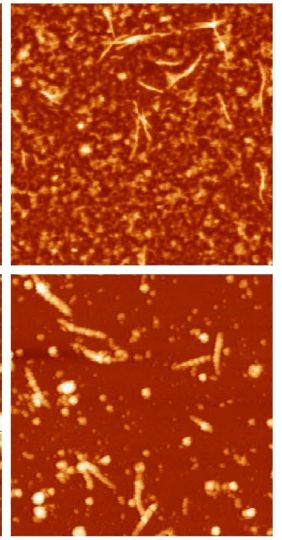

DOPC $+20 \%$ chol

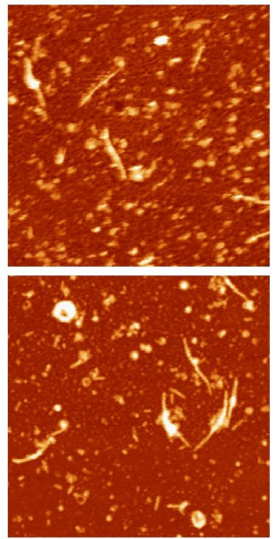

POPG $+20 \%$ chol

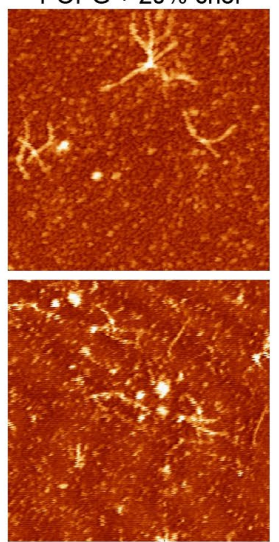

$3 \mathrm{~h}$
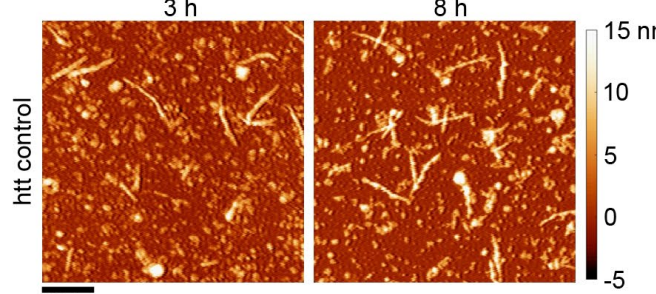

$\overline{500 \mathrm{~nm}}$
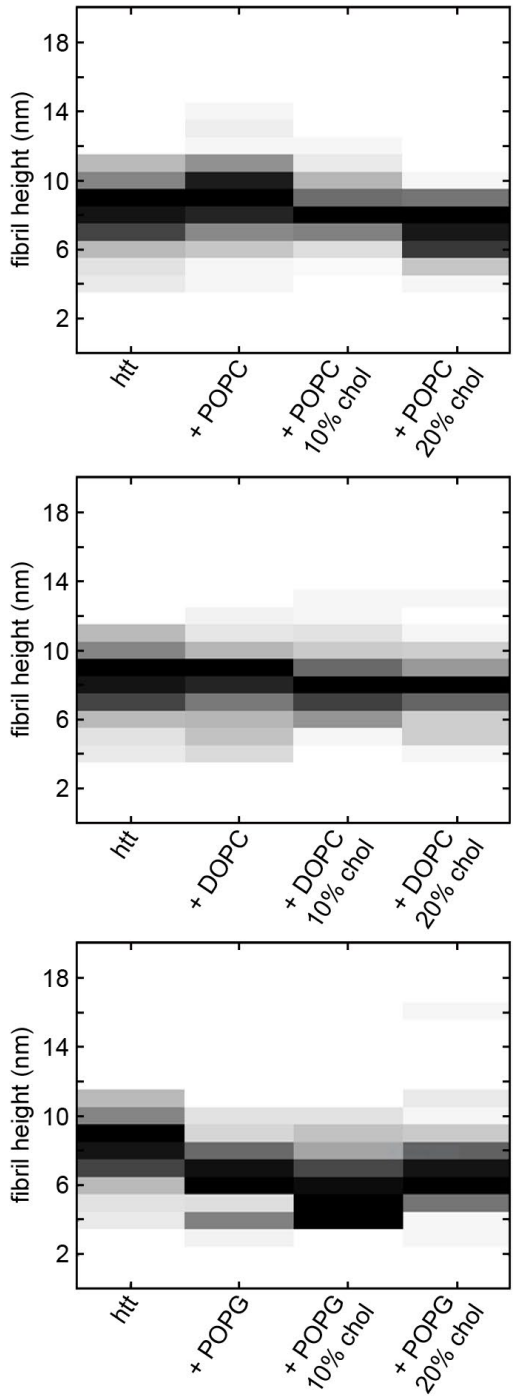

Figure 6.4 Representative AFM images showing htt aggregation after 3 and $8 \mathrm{~h}$ in the presence of no lipid, POPC, DOPC, or POPG vesicles with 0,10 , or $20 \%$ cholesterol. Height histograms of fibrils observed in AFM images after $8 \mathrm{~h}$ are included. 
The effect of lipid vesicles with increased cholesterol content on htt aggregation was different than the effect of the pure lipids for all three systems. Increased cholesterol levels were associated with decreased aggregation when compared to aggregation in the presence of the pure lipid system for POPC and POPG, while the opposite effect was observed with DOPC (Fig. 6.4, Fig. 6.5a and c). While the extent of fibrillization was influenced by membrane cholesterol content in all cases, the fibril morphology was similar across all POPC and DOPC conditions, indicating that the structure of the htt flanking regions in the structure did not change. ${ }^{35}$ However, with POPG, the decrease in the height of fibrils formed compared to htt alone is consistent with the results of Chapter 4 , and the fibrils also seem to get shorter with increased cholesterol content (Fig. 6.4). Further AFM analysis will need to be performed to analyze morphological changes, such as changes in the height and diameter of oligomeric aggregates.

Preliminary results also indicate that the physicochemical properties of the lipid system being spiked with cholesterol influence the effect on htt lipid binding (Fig. 6.4b-c). However, the lipid binding trends do not correlate to the aggregation trends across all lipid systems, highlighting the complexity of htt aggregation and indicating multiple mechanisms at play. Our PDA assays reveal that htt exhibits a lower affinity for POPC vesicles with up to $20 \%$ added cholesterol. Amphipathic $\alpha$-helices like the Nt17 domain of htt preferentially bind membranes with increased defects ${ }^{36-38}$ and the addition of cholesterol to POPC results in decreased fluidity and increased order, ${ }^{39}$ thus decreasing the occurrence of membrane defects. On the other hand, while the acyl chains of DOPC are compressed with the addition of cholesterol, the area per DOPC lipid headgroup increases, ${ }^{40}$ resulting in more defects at the bilayer surface and allowing for the observed increase in htt-DOPC interaction with increasing cholesterol content. Increasing cholesterol content also increased the interaction of htt with POPG lipid vesicles. Despite the 
electrostatic repulsion between POPG lipid headgroups, interlipid bridges and hydrogen bonding overcome the repulsion to result in a tightly packed bilayer. ${ }^{41}$ Cholesterol insertion into the membrane may spread out the POPG headgroups, limiting that strong interaction and allowing for increased interaction with htt. In addition, the cholesterol would decrease the charge density in the bilayer, which Chapter 4 results indicate could help facilitate htt insertion. As a future direction, MS studies should be performed to analyze the complexes formed between htt and the lipid systems as a result of increased cholesterol content, similar to the MS studies performed in Chapters 4 and 5.
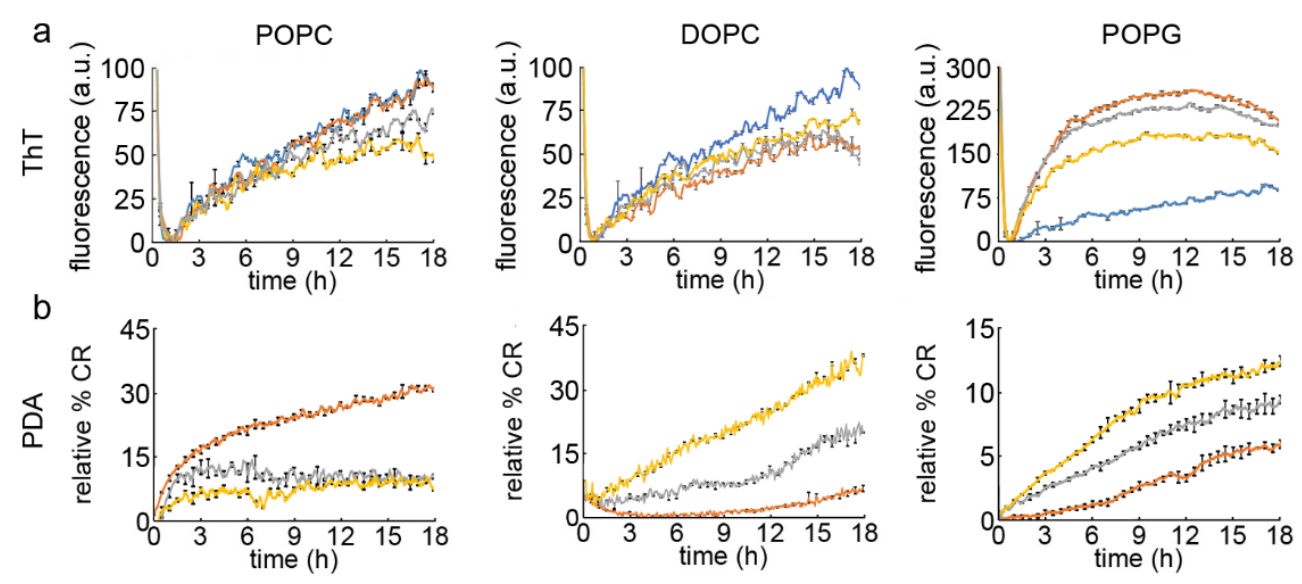

C

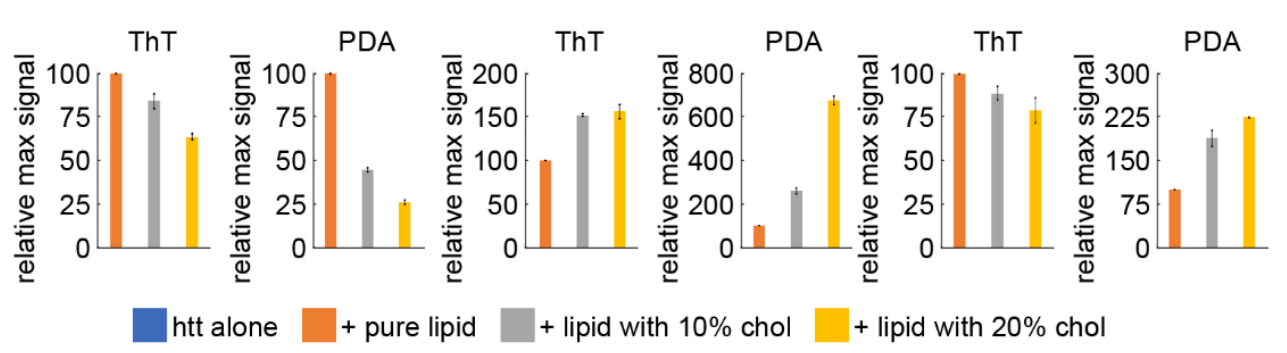

Figure 6.5 The impact of cholesterol on a membrane's effect on htt aggregation and lipid binding is dependent on the physicochemical properties of the pure lipid system. (a) ThT aggregation assays for htt-exon1(46Q) $(20 \mu \mathrm{M})$ in the presence of POPC, DOPC, or POPG with 0,10 , or $20 \%$ cholesterol. Error bars are provided for every sixth data point (30 $\mathrm{min}$ ) and represent the standard error of the mean (SEM). (b) PDA lipid binding assays of POPC, DOPC, or POPG with 0,10 , or $20 \%$ cholesterol exposed to htt-exon1(46Q). (c) The maximum ThT and PDA signal for each condition relative to the condition of the pure lipid system (set to 100). Error bars represent SEM. 
In previous studies, increasing cholesterol content of total brain lipid extract (TBLE) systems decreased the ability of htt to permeabilize the vesicles and altered the morphology of aggregates. ${ }^{8}$ Membrane disruption by amyloid proteins is theorized to be a mechanism associated with many neurodegenerative diseases like Alzheimer's Disease and Huntington's Disease, so understanding factors that decrease membrane damage and permeabilization could lead to potential therapeutic approaches that focus on preventing toxic interactions. Previous studies of htt aggregation in the presence of TBLE enriched with cholesterol used cholesterol levels that exceeded what is observed in a typical cellular membrane. ${ }^{8}$ Determining the effect of physiological concentrations of cholesterol in different pure lipid systems would lead to a better understanding of how cholesterol homeostasis influences htt binding to and aggregation on different lipid membranes.

\subsection{Identifying the interaction of specific aggregate species with lipid membranes}

The interaction of htt with lipid membranes is associated with a number of cytotoxic effects such as disrupted trafficking, ${ }^{13}$ organelle dysfunction, ${ }^{4,42,43}$ and neuronal death. ${ }^{44}$ However, due to the complex and heterogeneous nature of htt aggregation, it is difficult to pinpoint which specific aggregate species are responsible for the detrimental effects on membranes. In addition, aggregate species may exhibit a higher affinity for membranes with specific physicochemical properties, potentially determining the affinity and specificity of htt

toxic mechanisms. In Chapter 4, PDA/lipid binding assays revealed that the affinity of mutant htt for lipid membranes changes with lipid composition. Interestingly, the membrane affinity was also influenced by htt concentration. At low htt concentrations, htt exhibited a higher affinity for the negatively charged membranes (POPG and POPS), while a higher affinity for zwitterionic lipids (POPC and POPE) was observed at higher htt concentrations. The change in membrane 
affinity with htt concentration may be due to the population of aggregate species formed at each condition. As our PDA assays are kinetic assays that run for $12+$ hours, extensive htt aggregation occurs throughout the experiment. Htt aggregation is dependent on concentration, with increased concentration resulting in shortened lag phase, increased aggregation rate, and an overall increase in aggregate formation. ${ }^{45}$ With enhanced aggregate formation altering overall httmembrane association, this indicates that membrane affinity may vary between the various aggregate species. For example, increased affinity of htt for POPC with increased concentration, and thus increased aggregation, may indicate that higher-order aggregate species interact directly with the vesicles. Understanding the lipid properties that determine htt aggregate membrane affinity could help explain the targeted toxic effects of mutant htt on organelles with different membrane compositions.

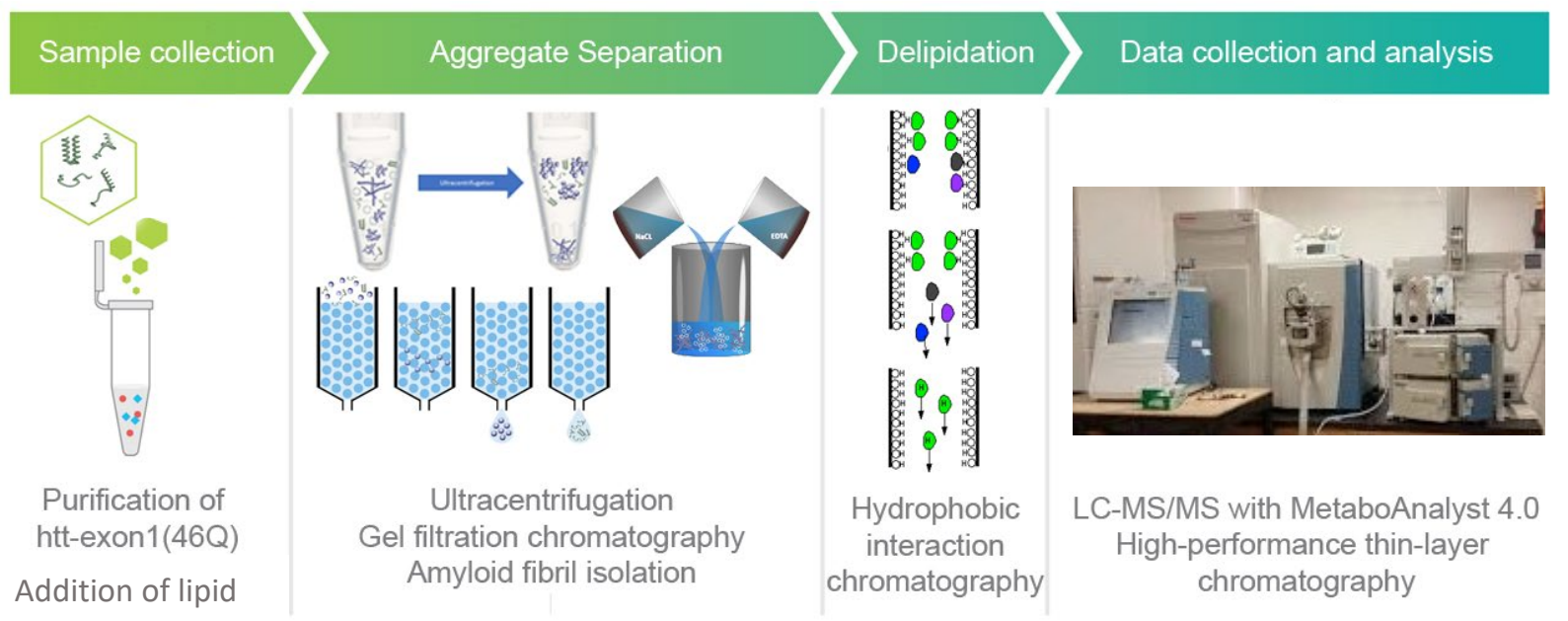

Figure 6.6 A proposed experimental protocol for studying the association of specific htt aggregates with lipids of varying physicochemical properties. Htt-exon1(46Q) will be purified and incubated with lipid vesicles, and the mixture will then undergo extensive separation procedures to result in separate monomer, oligomer, fibril, and lipid vesicle samples. Htt species will be separated from associated lipids with hydrophobic interaction chromatography, and lipid populations will be analyzed with LC-MS/MS. 
In order to determine the association of specific aggregate species with different lipid systems, a protocol must be developed to separate and analyze the complex mixture of species formed during htt aggregation in the presence of the lipids (Fig. 6.6). This can be accomplished through a combination of separation techniques. First, ultracentrifugation could be used to pellet the fibrils and free-lipid species, leaving the monomers and oligomers in solution. The monomers and oligomers could then be separated via gel filtration chromatography on Sephacryl S1000 resin, which has been used to successfully separated htt-exon1(46Q) monomers and oligomers with a resolving range of 20-300 nm. ${ }^{46}$ To separate the fibrils from the free lipid species pelleted during ultracentrifugation, amyloid fibril isolation could be performed as previously described. ${ }^{47}$ The combination of applied separation techniques will result in four samples of fully separated monomers, oligomers, fibrils, and lipid vesicles (Fig. 6.7). One problem when performing separation techniques on amyloid samples is that each of the samples will continue to aggregate after the separation is complete, potentially changing the population of aggregate species. However, in this case, further aggregation of each sample is not problematic as the lipid content of the entire sample represents only lipids that were associated with the originally separated aggregate species. Therefore, samples would undergo delipidation via hydrophobic interaction chromatography ${ }^{48}$ and the lipid content associated with each aggregate species could be analyzed using an MS lipidomic protocol. ${ }^{49}$ 


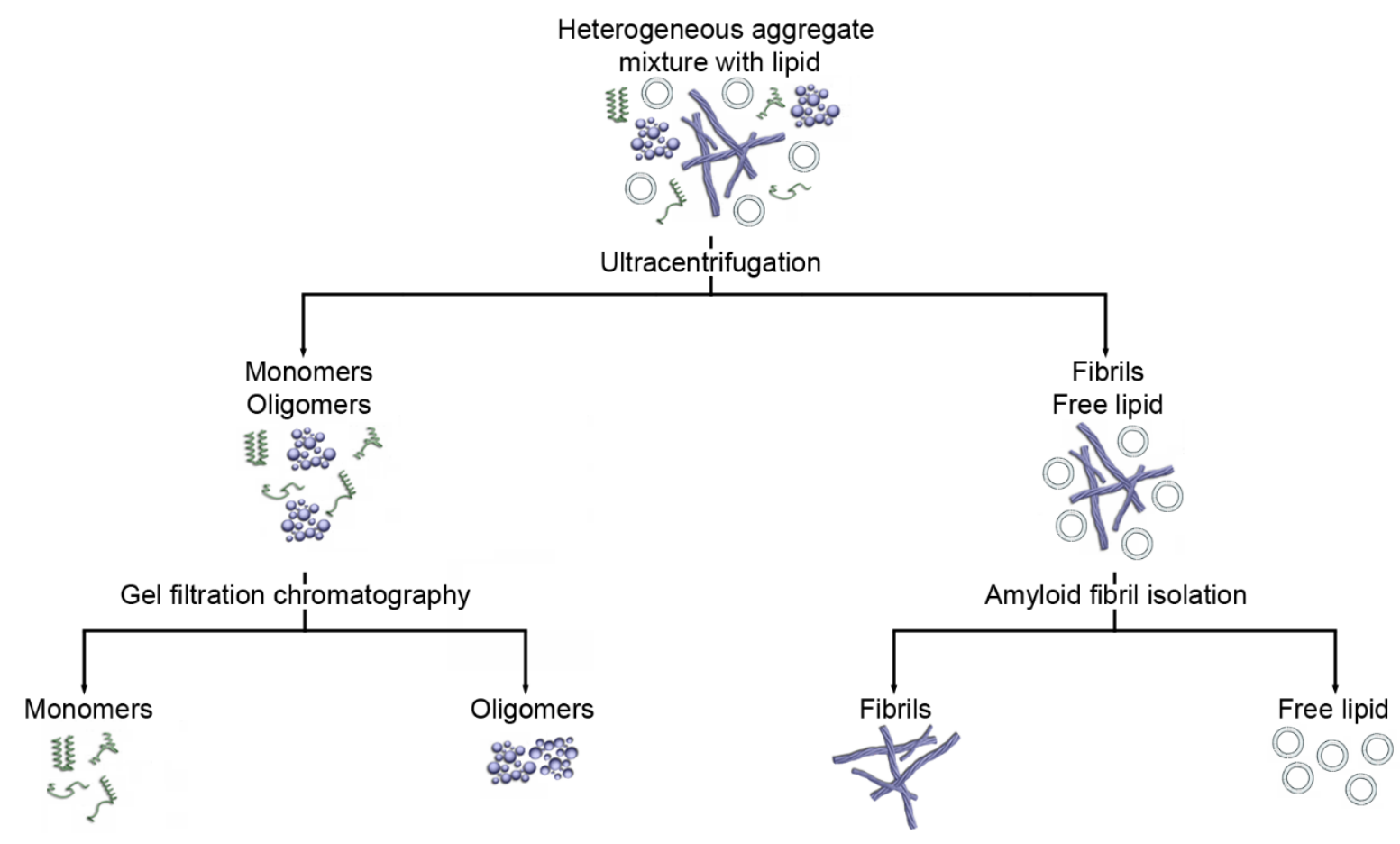

Figure 6.7 A schematic for the separation protocol of htt aggregates and lipid vesicles.

While this proposed separation protocol would work well for samples of htt alone, the separation becomes more complex due to the presence of the lipid vesicles. Htt aggregates accumulate and incorporate lipids into their structures, ${ }^{4,50-52}$ which could result in the additional mass causing protein aggregates to pellet with the lipid vesicles during the ultracentrifugation step. In this situation, the current proposed separation protocol would not account for the aggregate species associated or incorporated into the lipid vesicles, bringing about misleading results about relative aggregate affinity for membranes. This can be accounted for by separating the vesicles from the fibrils in the pellet formed during ultracentrifugation, then performing native gel electrophoresis, Western Blots, and densitometry on the aggregates incorporated into the lipid vesicles. The Western Blots can be stained with antibodies for htt monomers (MW1) ${ }^{53}$ and oligomers (A11). ${ }^{54}$ Densitometry can be utilized to determine the concentration of each aggregate species associated with the lipid vesicles, allowing for a relative comparison of 
aggregate affinity for specific lipid systems. Another complexity added by the presence of lipid membranes is the potential for aggregation in the bilayer and in solution. Htt can aggregate on the bilayer via lateral diffusion, ${ }^{7}$ so it is difficult to know if oligomers associated with lipid vesicles formed in solution and then bound the vesicle, or if they formed as a result of aggregation on the bilayer. To account for this, single-molecule microscopy can be used to monitor aggregation and diffusion of individual species in a lipid bilayer as has been done with $\mathrm{A} \beta .^{55}$ Total internal reflection fluorescence (TIRF) allows for the monitoring of aggregation on a bilayer, as well as the mobility of membrane-bound aggregate species to determine relative binding affinities. Using this technique, the extent of htt aggregation in the bilayer - as opposed to in solution - can be observed with membranes of different physicochemical properties.

Due to the complexity of htt aggregation, it is challenging to identify the specific aggregate species responsible for toxic effects on lipid membranes. In addition to the aggregate species most responsible, the mechanism of htt-induced membrane damage is largely unknown. Membrane damage could occur via the interaction of toxic aggregate species with the membrane, or via the aggregation of htt in the bilayer. It is possible that there are several toxic mechanisms at work. The contribution from this work is significant because it will provide insight into htt's interaction with lipid membranes that results in membrane damage and organelle dysfunction, with an additional focus on the membrane affinity of specific aggregate species. Knowledge gained from this study would broaden the understanding of organelle dysfunction in HD progression and potentially reveal specific interactions as a target for therapeutics.

\subsection{A systematic approach to understand amphipathic $\alpha$-helix membrane selectivity}

Much like htt, numerous proteins have domains that undergo a conformational transition from intrinsically disordered to an amphipathic $\alpha$-helix in the presence of binding partners. ${ }^{56}$ 
Many of these proteins are associated with critical functions that depend on the selective interaction of the $\alpha$-helical domain with specific membranes. ${ }^{38,57,58}$ Mechanisms have been proposed for the selective binding of AHs to particular membranes, however many of these mechanisms rely on an unbalanced helix with one poorly developed face, whether it be the hydrophobic or hydrophilic face. ${ }^{37,59,60}$ A poorly developed hydrophobic face is characterized by an abundance of small, weakly hydrophobic residues, while a poorly developed hydrophilic face lacks charged residues. ${ }^{60}$ One well-studied motif, the amphipathic lipid packing sensor (ALPS), has only large residues on its hydrophobic face and lacks charged residues. ${ }^{38,61} \mathrm{Nt} 17$, the $\mathrm{AH}$ utilized in our MD studies in Chapter 5, has two well-developed faces, as the hydrophobic face includes large and small residues and the hydrophilic face includes numerous charged, polar residues. Our simulations showed that Nt17's approach and initial interactions with the three bilayer systems indicated a correlation between the size of bilayer defects available and the size of the amino acid residue that initiated the interaction. The results obtained in Chapter 5 may have a broader impact on the field of protein-lipid interactions, as they reveal a potential mechanism for the selective membrane binding of AH-containing proteins that do not fall into the specific classification of containing a poorly developed face.

In order to thoroughly investigate the properties of $\mathrm{AH}$ sequences that influence membrane affinity, a large screen of the interactions between short de novo peptides and lipid vesicles with varying physicochemical properties could be performed. In a study like this, the peptides could be based on the AH sequence of three well-studied proteins: GMAP,$\alpha-$ synuclein, and htt. The AH of $\mathrm{GMAP}_{\mathrm{N}}$ has a poorly developed polar face, forcing it to rely on hydrophobic forces for interaction with membranes, while $\alpha$-synuclein largely depends on electrostatic interactions due to numerous charged residues and a poorly developed hydrophobic 
face. ${ }^{60} \mathrm{Nt} 17$, on the other hand, will present a system that balances the two forces. To determine the effect of certain sequence properties on the membrane affinity of these peptides, systematic amino acid substitutions will be performed. For example, to determine if amino acid size is a determinant in membrane affinity, large hydrophobic amino acids at the N-terminus of $\mathrm{GMAP}_{\mathrm{N}}$ could be replaced with smaller amino acids to determine if that increases the affinity of the peptide for lipid systems will smaller defects. In addition, the small amino acids on the hydrophobic face of $\alpha$-synuclein could also be replaced for similar studies. To study the effect of the polar face, amino acid substitutions could be performed that systematically add or remove charge to peptides.

In order to fully study the effects of amino acid size and charge on AH membrane affinity, many sequence combinations must be examined. For this screen, New England Peptide's custom peptide arrays ${ }^{62}$ can be utilized to perform high-throughput PDA assay to compare the affinity of AH-forming peptides for different lipid systems as developed in Chapter 2. This proprietary peptide array platform includes 96 unique, individually synthesized shortsequence peptides in a 96-well format that reduces the chances of cross-contamination. ${ }^{62}$ For the lipid components, three lipids with the same headgroup but different fluidity and size/propensity of bilayer defects could be utilized like in Chapter 5 (DMPC, POPC, DOPC). The PDA assay would reveal the impact of certain sequence changes to the peptide's affinity for different lipid vesicles, which could then be linked back to the physicochemical properties of the lipids. Based on results obtained from the PDA screen, WESTPA MD simulations could be performed as in Chapter 5 to study the modified peptides' method of approach to each bilayer system and see if it changes with amino acid size. 


\subsection{Conclusion}

Huntington's Disease is a fatal neurodegenerative order that is caused by an expanded glutamine repeat domain of htt, resulting in the formation of toxic aggregate species. Recent

therapeutic strategies have focused on inhibiting the aggregation and fibrillization of htt, ${ }^{63-65}$ but so far these efforts have remained unsuccessful in identifying a cure or treatment for HD. The results presented in this dissertation reveal that the physicochemical properties of lipid membranes influence htt aggregation, lipid binding, and the efficiency of aggregation inhibiting molecules that could influence future directions of HD therapeutic approaches.

In Chapter 3, the efficiency of two proven htt aggregation inhibitors was examined in the presence of lipid membranes. While EGCG remained effective, the effectiveness of curcumin in inhibiting htt aggregation was eliminated in the presence of lipids. In addition, the effect of the lipids on curcumin effectiveness was dependent on composition, as curcumin actually increased aggregation in the presence of one lipid system. These results highlight the complexity of htt aggregation in the presence of different membranes and indicated a different mechanism of membrane association and aggregation with the two membranes tested. This led to Chapters 4 and 5, where we aimed to determine the properties of lipid membranes that influence the mechanisms of htt aggregation and lipid binding. Fluidity was identified as a contributing property, with htt preferentially associating with more fluid membranes. In addition, an overall negative charge of membranes disrupts the insertion of htt into lipid vesicles, resulting in localization of $\mathrm{htt}$ at the bilayer surface that encourages rapid fibrillization. Finally, the size and propensity of membrane defects influences the mechanism of Nt17's approach and initial interaction with membranes of different composition. Combined, these results reveal details of 
the complex mechanisms of htt that are influenced by membrane properties with implications for normal htt function, toxic membrane interactions, and htt aggregate formation.

In addition to the advancement of knowledge about htt mechanisms, techniques developed and results obtained during this series of studies has the potential to advance the broader field of protein-lipid interactions. In Chapter 2, the developed normalized PDA lipid binding assay can be applied to measure and quantitatively compare the membrane affinity of pharmaceuticals, proteins, small molecules, and much more. While neither is a new technique, the combination of AFM and native MS complex analysis presents a novel approach to linking initial protein-lipid interactions to downstream aggregation that can be utilized with amyloid forming proteins such as A $\beta$ (Alzheimer's Disease) and $\alpha$-synuclein (Parkinson's Disease). Finally, the results presented in Chapter 5 indicate that both residue size and membrane defect size play a role in determining amphipathic $\alpha$-helix (AH) membrane affinity, a mechanism that is likely in play with other biologically relevant membrane-binding AHs.

Overall, the results presented here provide details about the mechanisms of mutant htt in HD progression and reveal the challenges of inhibiting aggregation in a physiologically relevant environment. Together, these studies highlight the important factors of htt lipid binding mechanisms. Despite the new knowledge gained about the factors influencing htt membrane affinity, further studies are required to link the association of specific aggregate species and complex membrane systems with toxicity. The identification of the toxic species and mechanisms involved in the interaction of htt with subcellular membranes will present novel targets for HD therapeutic development. 


\subsection{References}

1. Velier, J. et al. Wild-Type and Mutant Huntingtins Function in Vesicle Trafficking in the Secretory and Endocytic Pathways. Experimental Neurology 152, 34-40 (1998).

2. Davies, S. W. et al. Formation of neuronal intranuclear inclusions underlies the neurological dysfunction in mice transgenic for the HD mutation. Cell 90, 537-548 (1997).

3. Mihm, M. J. et al. Cardiac dysfunction in the R6/2 mouse model of Huntington's disease. Neurobiol Dis 25, 297-308 (2007).

4. Suopanki, J. et al. Interaction of huntingtin fragments with brain membranes - clues to early dysfunction in Huntington's disease. Journal of Neurochemistry 96, 870-884 (2006).

5. Wei, M. et al. A polydiacetylene-based fluorescence assay for the measurement of lipid membrane affinity. RSC Adv. 5, 66420-66425 (2015).

6. Zheng, F., Wu, Z. \& Chen, Y. A quantitative method for the measurement of membrane affinity by polydiacetylene-based colorimetric assay. Analytical Biochemistry 420, 171-176 (2012).

7. Pandey, N. K. et al. The 17-residue-long N terminus in huntingtin controls stepwise aggregation in solution and on membranes via different mechanisms. J. Biol. Chem. 293, 2597-2605 (2018).

8. Gao, X. et al. Cholesterol Modifies Huntingtin Binding to, Disruption of, and Aggregation on Lipid Membranes. Biochemistry 55, 92-102 (2016).

9. Chaibva, M. et al. Sphingomyelin and GM1 Influence Huntingtin Binding to, Disruption of, and Aggregation on Lipid Membranes. ACS Omega 3, 273-285 (2018).

10. Hands, S. L. \& Wyttenbach, A. Neurotoxic protein oligomerisation associated with polyglutamine diseases. Acta Neuropathol. 120, 419-437 (2010).

11. Ehrnhoefer, D. E. et al. Green tea (-)-epigallocatechin-gallate modulates early events in huntingtin misfolding and reduces toxicity in Huntington's disease models. Human Molecular Genetics 15, 2743-2751 (2006).

12. Truant, R., Atwal, R. \& Burtnik, A. Hypothesis: Huntingtin may function in membrane association and vesicular trafficking. Biochem. Cell Biol. 84, 912-917 (2006).

13. Chang, D. T. W., Rintoul, G. L., Pandipati, S. \& Reynolds, I. J. Mutant huntingtin aggregates impair mitochondrial movement and trafficking in cortical neurons. Neurobiology of Disease 22, 388-400 (2006).

14. Suopanki, J. et al. Interaction of huntingtin fragments with brain membranes - clues to early dysfunction in Huntington's disease. Journal of Neurochemistry 96, 870-884 (2006). 
15. Saher, G. et al. High cholesterol level is essential for myelin membrane growth. Nature Neuroscience 8, 468-475 (2005).

16. Maxfield, F. R. \& Tabas, I. Role of cholesterol and lipid organization in disease. Nature 438, 612-621 (2005).

17. Pfrieger, F. W. Role of cholesterol in synapse formation and function. Biochim. Biophys. Acta 1610, 271-280 (2003).

18. Liu, Q. et al. Neuronal LRP1 knockout in adult mice leads to impaired brain lipid metabolism and progressive, age-dependent synapse loss and neurodegeneration. J. Neurosci. 30, 17068-17078 (2010).

19. Yu, X. \& Zheng, J. Cholesterol promotes the interaction of Alzheimer $\beta$-amyloid monomer with lipid bilayer. J. Mol. Biol. 421, 561-571 (2012).

20. Burger, K., Gimpl, G. \& Fahrenholz, F. Regulation of receptor function by cholesterol. CMLS, Cell. Mol. Life Sci. 57, 1577-1592 (2000).

21. Thiele, C., Hannah, M. J., Fahrenholz, F. \& Huttner, W. B. Cholesterol binds to synaptophysin and is required for biogenesis of synaptic vesicles. Nature Cell Biology 2, 42 49 (2000).

22. Mauch, D. H. et al. CNS Synaptogenesis Promoted by Glia-Derived Cholesterol. Science 294, 1354-1357 (2001).

23. Serfis, A. B., Brancato, S. \& Fliesler, S. J. Comparative behavior of sterols in phosphatidylcholine-sterol monolayer films. Biochimica et Biophysica Acta (BBA) Biomembranes 1511, 341-348 (2001).

24. Shamitko-Klingensmith, N., Molchanoff, K. M., Burke, K. A., Magnone, G. J. \& Legleiter, J. Mapping the Mechanical Properties of Cholesterol-Containing Supported Lipid Bilayers with Nanoscale Spatial Resolution. Langmuir 28, 13411-13422 (2012).

25. Valenza, M. et al. Dysfunction of the Cholesterol Biosynthetic Pathway in Huntington's Disease. J Neurosci 25, 9932-9939 (2005).

26. Valenza, M. et al. Cholesterol biosynthesis pathway is disturbed in YAC128 mice and is modulated by huntingtin mutation. Hum. Mol. Genet. 16, 2187-2198 (2007).

27. Valenza, M. et al. Progressive dysfunction of the cholesterol biosynthesis pathway in the R6/2 mouse model of Huntington's disease. Neurobiol. Dis. 28, 133-142 (2007).

28. Ritch, J. J. et al. Multiple Phenotypes in Huntington Disease Mouse Neural Stem Cells. Mol Cell Neurosci 50, 70-81 (2012).

29. Trushina, E. et al. Mutant huntingtin inhibits clathrin-independent endocytosis and causes accumulation of cholesterol in vitro and in vivo. Hum Mol Genet 15, 3578-3591 (2006). 
30. Luthi-Carter, R. et al. SIRT2 inhibition achieves neuroprotection by decreasing sterol biosynthesis. PNAS 107, 7927-7932 (2010).

31. del Toro, D. et al. Altered cholesterol homeostasis contributes to enhanced excitotoxicity in Huntington's disease. J. Neurochem. 115, 153-167 (2010).

32. Dietschy, J. M. \& Turley, S. D. Thematic review series: brain Lipids. Cholesterol metabolism in the central nervous system during early development and in the mature animal. J. Lipid Res. 45, 1375-1397 (2004).

33. Zacco, A. et al. 3-hydroxy-3-methylglutaryl coenzyme A reductase inhibitors protect cortical neurons from excitotoxicity. J. Neurosci. 23, 11104-11111 (2003).

34. Valenza, M. \& Cattaneo, E. Cholesterol dysfunction in neurodegenerative diseases: is Huntington's disease in the list? Prog. Neurobiol. 80, 165-176 (2006).

35. Lin, H.-K. et al. Fibril polymorphism affects immobilized non-amyloid flanking domains of huntingtin exon1 rather than its polyglutamine core. Nat Commun 8, 1-12 (2017).

36. Chaibva, M., Burke, K. A. \& Legleiter, J. Curvature Enhances Binding and Aggregation of Huntingtin at Lipid Membranes. Biochemistry 53, 2355-2365 (2014).

37. Cui, H., Lyman, E. \& Voth, G. A. Mechanism of Membrane Curvature Sensing by Amphipathic Helix Containing Proteins. Biophys J 100, 1271-1279 (2011).

38. Drin, G. et al. A general amphipathic alpha-helical motif for sensing membrane curvature. Nat. Struct. Mol. Biol. 14, 138-146 (2007).

39. Schumann-Gillett, A. \& O'Mara, M. L. The effects of oxidised phospholipids and cholesterol on the biophysical properties of POPC bilayers. Biochimica et Biophysica Acta (BBA) Biomembranes 1861, 210-219 (2019).

40. Alwarawrah, M., Dai, J. \& Huang, J. A Molecular View of the Cholesterol Condensing Effect in DOPC Lipid Bilayers. J Phys Chem B 114, 7516-7523 (2010).

41. Zhao, W., Róg, T., Gurtovenko, A. A., Vattulainen, I. \& Karttunen, M. Atomic-Scale Structure and Electrostatics of Anionic Palmitoyloleoylphosphatidylglycerol Lipid Bilayers with $\mathrm{Na}+$ Counterions. Biophysical Journal 92, 1114-1124 (2007).

42. Eckmann, J. et al. Mitochondrial Membrane Fluidity is Consistently Increased in Different Models of Huntington Disease: Restorative Effects of Olesoxime. Mol Neurobiol 50, 107-118 (2014).

43. Choo, Y. S., Johnson, G. V. W., MacDonald, M., Detloff, P. J. \& Lesort, M. Mutant huntingtin directly increases susceptibility of mitochondria to the calcium-induced permeability transition and cytochrome c release. Hum. Mol. Genet. 13, 1407-1420 (2004). 
44. Ruan, Q., Lesort, M., MacDonald, M. E. \& Johnson, G. V. W. Striatal cells from mutant huntingtin knock-in mice are selectively vulnerable to mitochondrial complex II inhibitorinduced cell death through a non-apoptotic pathway. Hum. Mol. Genet. 13, 669-681 (2004).

45. Scherzinger, E. et al. Self-assembly of polyglutamine-containing huntingtin fragments into amyloid-like fibrils: implications for Huntington's disease pathology. Proc. Natl. Acad. Sci. U.S.A. 96, 4604-4609 (1999).

46. Ormsby, A. R., Ramdzan, Y. M., Mok, Y.-F., Jovanoski, K. D. \& Hatters, D. M. A Platform to View Huntingtin Exon 1 Aggregation Flux in the Cell Reveals Divergent Influences from Chaperones hsp40 and hsp70. J Biol Chem 288, 37192-37203 (2013).

47. Tennent, G. A. [2] Isolation and characterization of amyloid fibrils from tissue. in Methods in Enzymology vol. 309 26-47 (Elsevier, 1999).

48. Velkov, T., Lim, M. L. R., Capuano, B. \& Prankerd, R. A protocol for the combined subfractionation and delipidation of lipid binding proteins using hydrophobic interaction chromatography. Journal of Chromatography B 867, 238-246 (2008).

49. Gellermann, G. P. et al. Raft lipids as common components of human extracellular amyloid fibrils. Proc Natl Acad Sci U S A 102, 6297-6302 (2005).

50. Valencia, A. et al. Mutant huntingtin and glycogen synthase kinase 3-beta accumulate in neuronal lipid rafts of a presymptomatic knock-in mouse model of Huntington's disease. $J$. Neurosci. Res. 88, 179-190 (2010).

51. Qin, Z.-H. et al. Huntingtin bodies sequester vesicle-associated proteins by a polyprolinedependent interaction. J. Neurosci. 24, 269-281 (2004).

52. Kegel, K. B. et al. Huntingtin expression stimulates endosomal-lysosomal activity, endosome tubulation, and autophagy. J. Neurosci. 20, 7268-7278 (2000).

53. Baldo, B. et al. TR-FRET-based duplex immunoassay reveals an inverse correlation of soluble and aggregated mutant huntingtin in huntington's disease. Chem. Biol. 19, 264-275 (2012).

54. Morozova, O. A., Gupta, S. \& Colby, D. W. Prefibrillar huntingtin oligomers isolated from HD brain potently seed amyloid formation. FEBS Letters 589, 1897-1903 (2015).

55. Chang, C.-C., Edwald, E., Veatch, S., Steel, D. G. \& Gafni, A. Interactions of amyloid- $\beta$ peptides on lipid bilayer studied by single molecule imaging and tracking. Biochimica et Biophysica Acta (BBA) - Biomembranes 1860, 1616-1624 (2018).

56. Segrest, J. P., Loof, H. D., Dohlman, J. G., Brouillette, C. G. \& Anantharamaiah, G. M. Amphipathic helix motif: Classes and properties. Proteins: Structure, Function, and Bioinformatics 8, 103-117 (1990). 
57. Giménez-Andrés, M., Čopič, A. \& Antonny, B. The Many Faces of Amphipathic Helices. Biomolecules 8, (2018).

58. Masuda, M. et al. Endophilin BAR domain drives membrane curvature by two newly identified structure-based mechanisms. EMBO J 25, 2889-2897 (2006).

59. Hatzakis, N. S. et al. How curved membranes recruit amphipathic helices and protein anchoring motifs. Nat. Chem. Biol. 5, 835-841 (2009).

60. Pranke, I. M. et al. $\alpha$-Synuclein and ALPS motifs are membrane curvature sensors whose contrasting chemistry mediates selective vesicle binding. J Cell Biol 194, 89-103 (2011).

61. Vanni, S. et al. Amphipathic Lipid Packing Sensor Motifs: Probing Bilayer Defects with Hydrophobic Residues. Biophys J 104, 575-584 (2013).

62. Custom Peptide Arrays | New England Peptide. https://www.newenglandpeptide.com/services/peptides/custom-peptide-arrays.

63. Kim, S. \& Kim, K.-T. Therapeutic Approaches for Inhibition of Protein Aggregation in Huntington's Disease. Exp Neurobiol 23, 36-44 (2014).

64. Zhang, X. et al. A potent small molecule inhibits polyglutamine aggregation in Huntington's disease neurons and suppresses neurodegeneration in vivo. Proc Natl Acad Sci U S A 102, 892-897 (2005).

65. Chaudhary, R. K., Patel, K. A., Patel, M. K., Joshi, R. H. \& Roy, I. Inhibition of Aggregation of Mutant Huntingtin by Nucleic Acid Aptamers In Vitro and in a Yeast Model of Huntington's Disease. Molecular Therapy 23, 1912-1926 (2015). 